\title{
Investigation of Low-Velocity Impact Damage in
}

\section{Fibre-Metal-Laminates}

\author{
by \\ Jeremy F. Laliberté, B.Eng.
}

\begin{abstract}
A thesis submitted to
the Faculty of Graduate Studies and Research

in partial fulfilment of the requirements for the degree of

Doctor of Philosophy
\end{abstract}

Ottawa-Carleton Institute for Mechanical and Aerospace Engineering

Department of Mechanical and Aerospace Engineering

Carleton University

Ottawa, Ontario

April 26, 2002

(C) Copyright

2002, Jeremy F. Laliberté 
National Library

of Canada

Acquisitions and Bibliographic Services

395 Wellington Street Ottawa ON K1A ON4 Canada
Bibliothèque nationale

du Canada

Acquisitions et

services bibliographiques

395, rue Wellington

Ottawa ON K1A ON4

Canada
The author has granted a nonexclusive licence allowing the National Library of Canada to reproduce, loan, distribute or sell copies of this thesis in microform, paper or electronic formats.
L'auteur a accordé une licence non exclusive permettant à la Bibliothèque nationale du Canada de reproduire, prêter, distribuer ou vendre des copies de cette thèse sous la forme de microfiche/film, de reproduction sur papier ou sur format électronique.

The author retains ownership of the copyright in this thesis. Neither the thesis nor substantial extracts from it may be printed or otherwise reproduced without the author's permission.
L'auteur conserve la propriété du droit d'auteur qui protège cette thèse. $\mathrm{Ni}$ la thèse ni des extraits substantiels de celle-ci ne doivent être imprimés ou autrement reproduits sans son autorisation. 
The undersigned hereby recommend to the Faculty of Graduate Studies and Research acceptance of the thesis,

\section{Investigation of Low-Velocity Impact Damage in Fibre-Metal-Laminates}

Submitted by

Jeremy F. Laliberté, B.Eng.

in partial fulfilment of the requirements

for the degree of Doctor of Philosophy

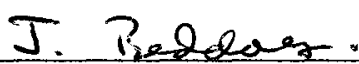

J. Beddoes, Chair, Department of Mechanical and Aerospace Engineering

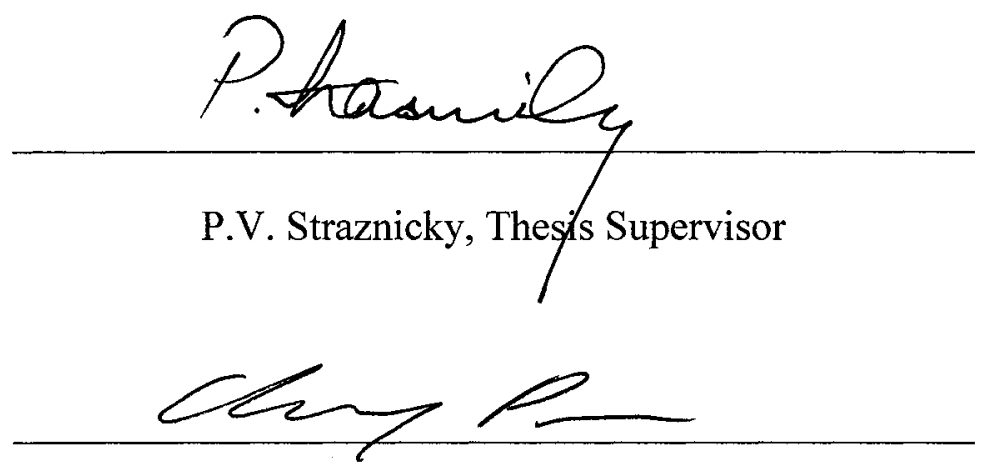

C. Poon, Thesis Co-supervisor

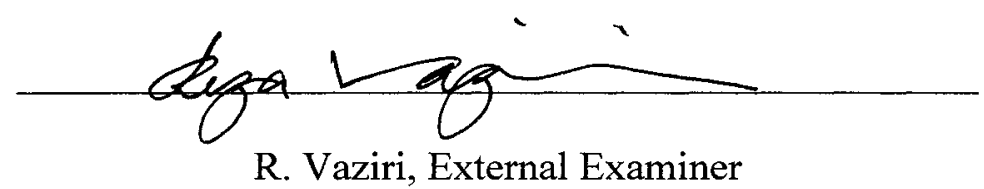

R. Vaziri, External Examiner

Carleton University

August 2, 2002 
Dedicated to the loving memory of my Grandfather, Norman Richardson.

Per Ardua Ad Astra 


\section{ABSTRACT}

Fibre-metal-laminates (FMLs) represent a significant evolution in airframe material technology. This new family of materials combines low density, high strength and excellent damage tolerance through the use of metal layers strengthened with fibrereinforced polymer layers. When subjected to low-velocity impact these laminates like traditional composites, develop internal delamination damage, matrix cracks and limited fibre fractures. Also, as in traditional composites, this damage is hidden within the laminate. A method for predicting the amount of internal damage would reduce the experimental testing requirements for the certification of new laminates.

This thesis describes the development of a modelling methodology that makes use of a new material subroutine based on continuum damage mechanics in the explicit finiteelement code LS-DYNA. This subroutine was verified using the experimental data from low-velocity impact tests of various types of GLARE (GLAss REinforced) aluminum laminates, a common type of commercially available fibre-metal-laminate. Static characterization tests were also conducted on GLARE coupons to provide basic property data for the development of the model. These included static tensile tests and double cantilever beam delamination tests. The modelling methodology was used to improve simulations of low-velocity impact on GLARE laminates. The simulations demonstrated that intralaminar damage has a greater effect on the impact response of the panels than interlaminar damage. Parts of this thesis were components of a multi-year collaborative FML Durability Project between Carleton University, Bombardier Aerospace and the National Research Council Canada. 


\section{ACKNOWLEDGEMENTS}

I would like to give thanks to the following individuals and organizations for their support and assistance throughout this Project:

- To my parents, Debbie and Andre for their support and encouragement throughout my undergraduate and graduate studies. To my wife, Kelly, for her patience and support, and for distracting me enough to preserve my sanity.

- To my supervisor, Professor Paul V. Straznicky of Carleton University for his wisdom, his patient review of my work and for providing an excellent role model as I begin my career. To my co-supervisor, Dr. Cheung Poon of NRC for his highly knowledgeable technical assistance, his guidance throughout this project and for giving me the opportunity to work at NRC.

- To all of my friends at Carleton and NRC, in particular Alex Jodoin and Andrew Johnston, for patiently listening to my gripes, grievances and grumbles.

- To the technical staff of the Institute for Aerospace Research of NRC for their help during the experimental portions of this project.

- To the Natural Sciences and Engineering Research Council for providing the majority of my $\mathrm{PhD}$ funding through a Post-Graduate Scholarship.

- To Brent Lievers and Jack van Hoof for their tireless assistance with LS-DYNA. 
- To Bombardier Aerospace and specifically Leo Kok, for providing funding and guidance for the FML Durability Project. To Bill Evancho of AEI for providing materials used in the experimental portions of this work.

- To Katherine McCuaig, an undergraduate student from Carleton University, who worked with me tirelessly for three summers at NRC helping to carry out many of the characterization experiments.

- To the staff of the Mechanical and Aerospace Engineering Departmental Office for their constant help with my undergraduate and graduate studies and the other endeavours I have undertaken over the years.

- Last but not least, to Carleton University, my home away from home for nine years, thank you. 


\section{TABLE OF CONTENTS}

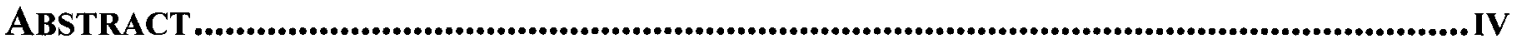

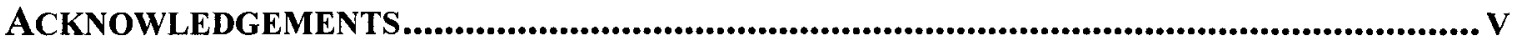

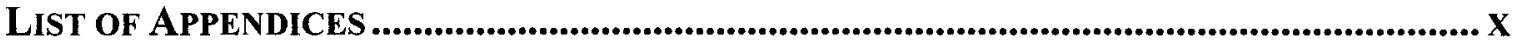

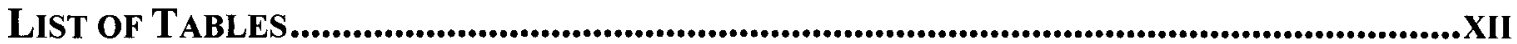

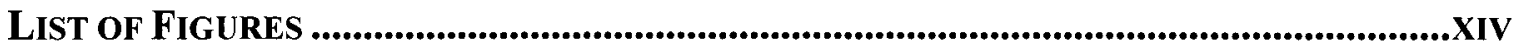

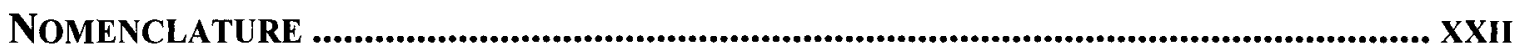

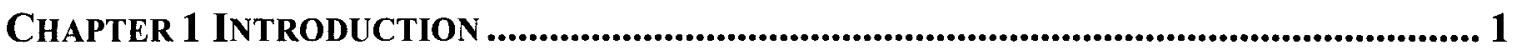

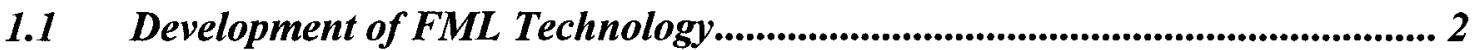

$1.2 \quad$ Mechanical Properties of Typical FMLs ................................................... 6

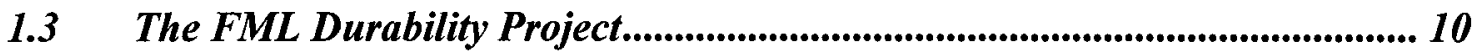

1.4 Thesis Motivation ............................................................................................... 13

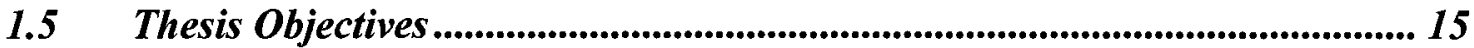

1.6 Thesis Overview........................................................................................................ 16

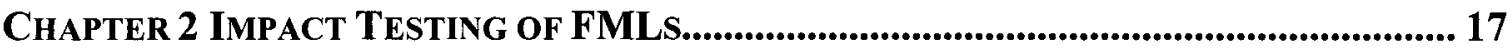

$2.1 \quad$ Review of Impact Testing of FMLs............................................................. 18

2.1.1 Coupon Impact Test Methods and Results............................................. 19

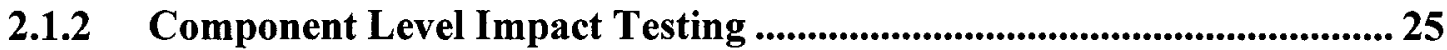

2.1.3 Impact Damage Measurement Techniques ............................................. 27

$2.2 \quad$ Low-Velocity Impact Testing of FMLs ............................................................. 29

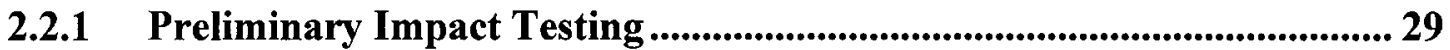

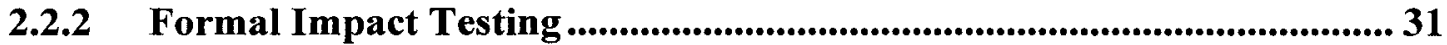

2.2.3 Experimental Procedure....................................................................... 32

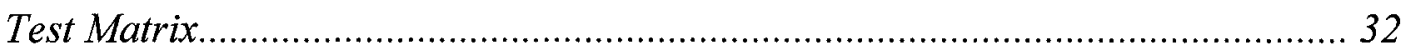

Drop Tower Data Collection Methods ........................................................ 32

Impact Damage Inspection..................................................................... 35 
2.2.4 Impact Test Results................................................................................................. 36

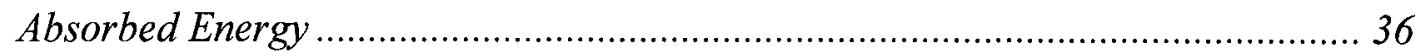

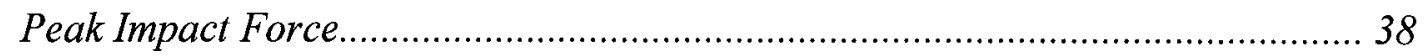

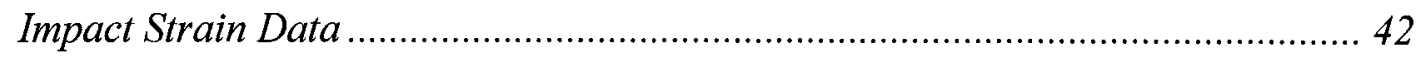

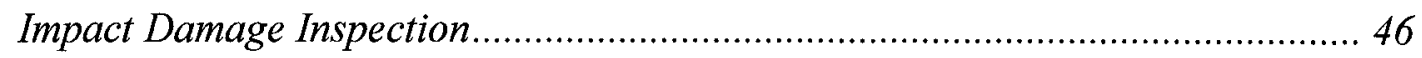

Chapter 3 FML IMPact Modelling MEthodology.................................................... 57

$3.1 \quad$ Review of Modelling of FMLs........................................................................... 58

3.1.1 Finite Element Modelling of FMLs ................................................................ 59

3.1.2 Analytical Modelling of Impact on FMLs...................................................... 61

3.1.3 Delamination Damage Modelling for FMLs............................................... 65

3.1.4 Delamination Modelling for Composites .................................................... 69

3.2 Proposed Modelling Methodology...................................................................... 70

3.3 Overview of the LS-DYNA Explicit Finite Element Code................................ 74

3.3.1 Thin-Shell Elements ................................................................................................ 76

3.3.2 Thick-Shell Elements ....................................................................................... 77

3.3.3 Solid-Brick Elements ............................................................................................ 80

3.3.4 Hourglass Control............................................................................................................ 81

3.3.5 Interface Models...................................................................................... 82

3.3.6 Material Models ............................................................................................. 84

3.3.7 Application of LS-DYNA to Model Impact Damage in FMLs ................. 86

Chapter 4 Simulations Of LOW-Velocity IMPACt ON ALUMinUm .......................... 87

$4.1 \quad$ Material Properties ................................................................................................................. 88

4.2 Mesh Density and Geometry ................................................................................. 89

4.3 Simulated Events...........................................................................................90

$4.4 \quad$ Preliminary Simulation Results.................................................................................. 93

4.5 Comparison to Experimental Data....................................................................... 96 
4.6 Final Simulations of Alumin um.................................................................... 97

4.6.1 Simulation Parameters ................................................................................. 97

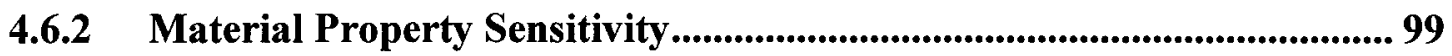

4.6.3 Results for the Final 2024-T3 Simulations............................................. 102

Chapter 5 Delamination Damage Characterization ....................................... 105

5.1 Review of Delamination Characterization of FMLs .................................... 106

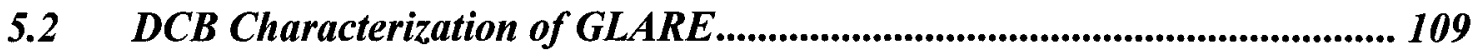

5.2.1 Preparation of Test Specimens ............................................................. 109

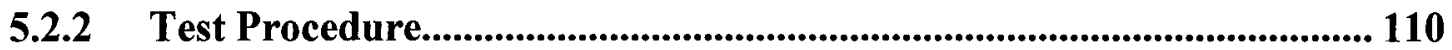

5.2.3 Delamination Test Results........................................................................ 110

5.2.4 Application of the DCB Test Results in LS-DYNA........................... 113

Chapter 6 Development of a CDM-Based Subroutine .................................... 114

6.1 Review of Continuum Damage Mechanics for Composites and FMLs....... 115

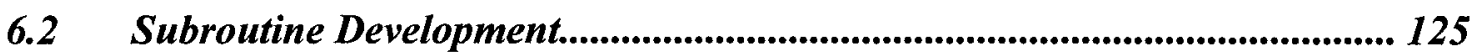

$6.3 \quad$ Validation of Elastic Model ................................................................. 130

6.4 Validation of the Damage Model...................................................................... 132

6.4.1 Static Tensile Testing of GLARE .................................................. 132

6.4.2 Tensile Simulations of GLARE in LS-DYNA .................................... 134

CHAPTER 7 Simulation OF LOW-VELOCITY IMPACTS ON GLARE LAMINATES ..... 141

7.1 Application of the FML Damage Modelling Methodology .......................... 142

7.2 GLARE Simulation Input Parameters .......................................................... 143

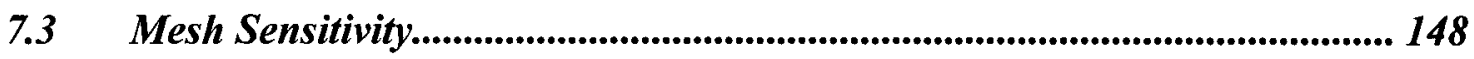

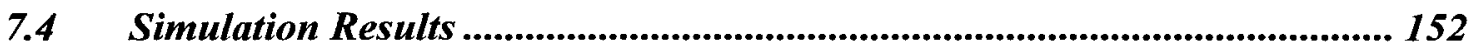

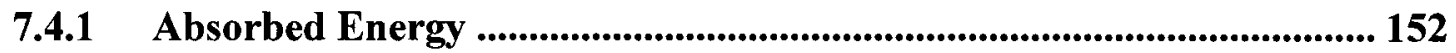

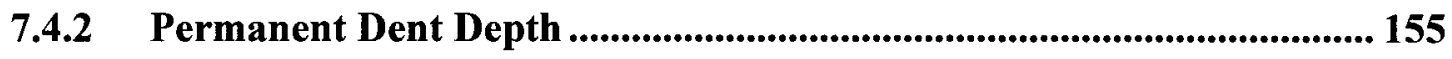

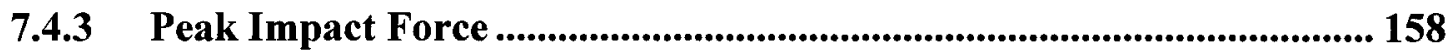

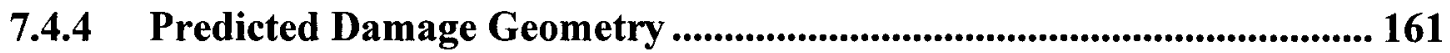


CHAPTER 8 SUMMARY AND DISCUSSION OF RESULTS ............................................... 169

$8.1 \quad$ Experimental Impact Testing ............................................................ 170

8.2 Damage Modelling Procedure for FMLs................................................ 173

8.2.1 Impact Damage Modelling of Aluminum ........................................... 173

8.2.2 Delamination Growth Characterization ............................................... 175

8.2.3 Development of Prepreg Damage Model .............................................. 176

$8.3 \quad$ Impact Modelling of GLARE ................................................................... 178

8.3.1 Mesh Sensitivity .......................................................................................... 178

8.3.2 Predicted Panel Response.......................................................................... 179

8.3.3 Predicted Damage Geometry ....................................................................... 184

8.4 Limitations of the Modelling Procedure ..................................................... 185

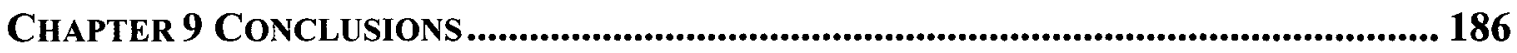

Chapter 10 Future Work ......................................................................................... 188

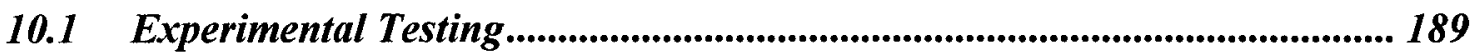

$10.2 \quad$ Full Failure Prediction of FMLs ......................................................... 190

10.3 Improved Delamination Modelling ..................................................................... 191

10.4 Miscellaneous Extensions and Additions to the Modelling Methodology... 192

10.5 Additional Applications...................................................................... 193

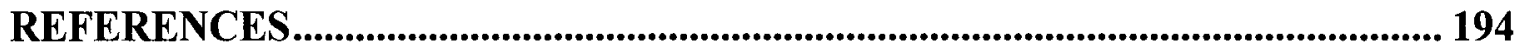




\section{LIST OF APPENDICES}

A - Preliminary Impact Test Results with NRC Fixture..................................... 205

B - Preliminary Impact Test Results with NASA ANd NRC Test Fixtures . 206

C - NRC AND NASA IMPACT FIXTURE RESULTS ......................................................... 207

D - FORMAL IMPACT TEST RESULTS................................................................................. 209

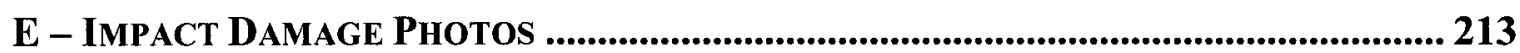

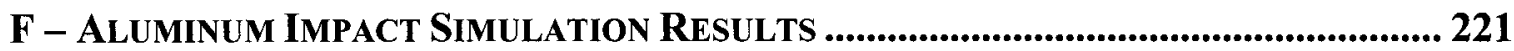

G - GLARE IMPACT SIMULATION RESULTS ..................................................................... 224

H - Partial list of Publications Resulting from the FML Project.............. 226 


\section{LIST OF TABLES}

Table 1: Summary of some commercially available FMLs........................................ 7

Table 2: Summary of properties of several FMLs and 2024-T3 aluminum .................... 8

Table 3: Summary of impact tests conducted to date on FMLs................................... 19

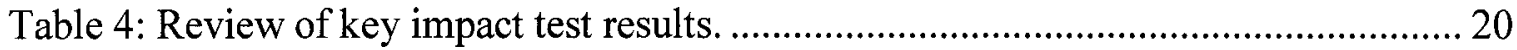

Table 5: Test matrix for formal FML impact tests.................................................. 32

Table 6: Summary of instrumented and impacted GLARE specimens. ......................... 35

Table 7: Summary of inspected specimens........................................................... 35

Table 8: Delamination measurements from the $\mathrm{x}$-rays and cross-sections..................... 50

Table 9: Summary of observed damage in the cross-sectioned GLARE panels. ............. 50

Table 10: Summary of impact test results for GLARE laminates. ............................... 56

Table 11: Summary of modelling techniques applied to FMLs..................................58

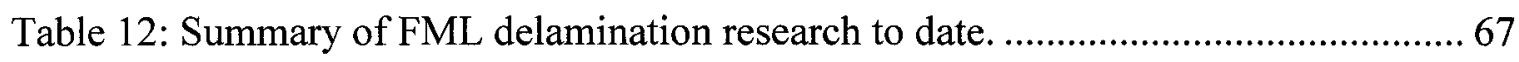

Table 13: Important features of LS-DYNA used in the simulation of GLARE and aluminum subjected to low-velocity impact.............................................. 75

Table 14: Units used throughout LS-DYNA simulations...................................... 76

Table 15: Summary of baseline material properties used for 2024-T3 .......................... 88

Table 16: Simulation matrix for simple aluminum impact events............................. 92

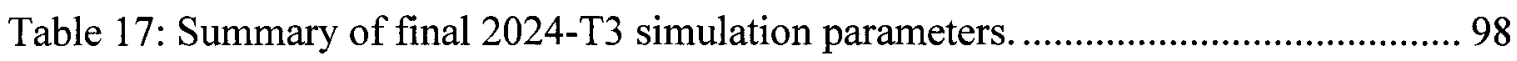

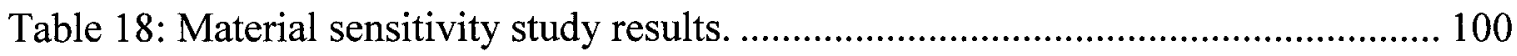

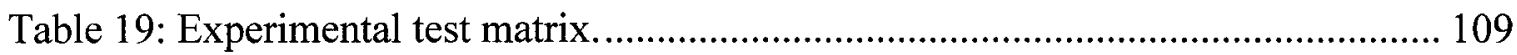

Table 20: Summary of shear rupture estimates and material properties..................... 128 
Table 21: Elastic properties of the F185/S2 prepreg material.

Table 22: Summary of static tensile results for the GLARE-3 test coupons. 133

Table 23: List of prepreg and GLARE-3-3/2 coupon simulations 136

Table 24: Summary of prepreg constituent properties 136

Table 25: Aluminum material properties

Table 26: Prepreg material input properties. 144

Table 27: Summary of GLARE low-velocity impact simulations. 147

Table 28: Summary of GLARE mesh density simulation parameters. 148

Table A1: Summary of preliminary results using the NRC impact fixture and various impactors. 205

Table B1: Preliminary impact test results using the NRC and NASA fixtures with $25 \mathrm{~mm}$ and $12.5 \mathrm{~mm}$ impactors, respectively 206

Table D1: Summary of GLARE-3 formal impact test results. 209

Table D2: Summary of GLARE-4 formal impact test results. 210

Table D3: Summary of GLARE-5 formal impact test results. 211

Table D4: Summary of aluminum formal impact test results. 212

Table F1: Summary of peak force results for preliminary aluminum simulations. .221

Table F2: Dent depth results for preliminary aluminum impact simulations. 222

Table F3: Impact energy results for preliminary aluminum impact simulations. 223

Table F4: Results of the final impact simulations on aluminum 2024-T3 223

Table G1: Summary of GLARE simulation absorbed energy results. 224

Table G2: Summary of GLARE simulation maximum force results 224

Table G3: Summary of GLARE permanent indentation results. 225 


\section{LIST OF FIGURES}

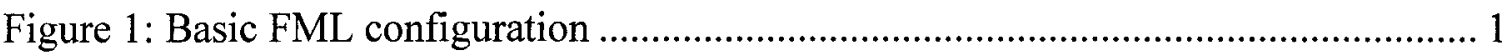

Figure 2: Variation in stress-intensity factors for layered and monolithic sheets............... 3

Figure 3: Aggregate multi-site fatigue crack propagation in GLARE-3-2/1 compared to

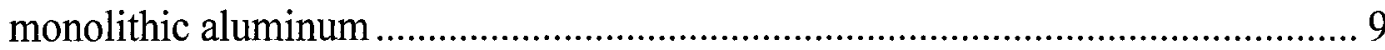

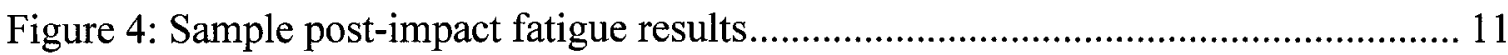

Figure 5: Areas of civil transport aircraft exposed to impact hazards. .............................. 14

Figure 6: Formation of impact damage in a traditional composite................................... 14

Figure 7: Basic impact test fixture geometries.......................................................... 18

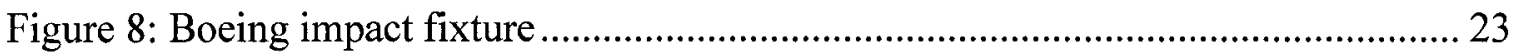

Figure 9: NASA 1092 impact fixture........................................................................... 23

Figure 10: Wright-Patterson impact apparatus ............................................................ 24

Figure 11: ARALL stiffened impact test panel.............................................................. 26

Figure 12: Comparison of damage geometry from the (a) NASA and (b) NRC impact

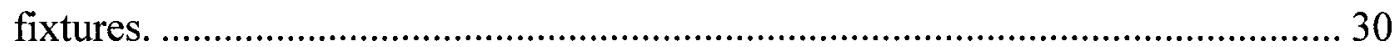

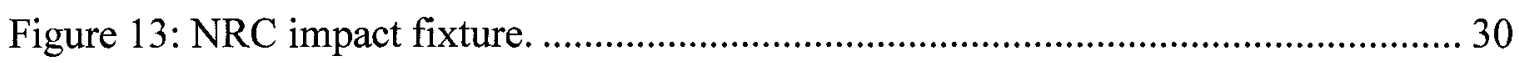

Figure 14: GLARE-2/1 variants subjected to impact testing............................................. 31

Figure 15: NRC Dynatup drop-weight impact tower with the NRC fixture..................... 33

Figure 16: Block diagram for Dynatup Model 730-I impact system................................. 33

Figure 17: Absorbed energy versus impact energy for GLARE and 2024-T3................ 37

Figure 18: Normalized absorbed energy versus impact energy ....................................... 38

Figure 19: Impact force versus impact velocity.............................................................. 39 
Figure 20: Aluminum 2024-T3 sample load traces.

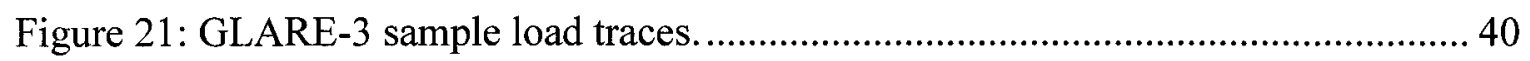

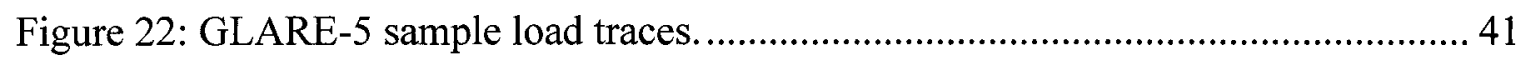

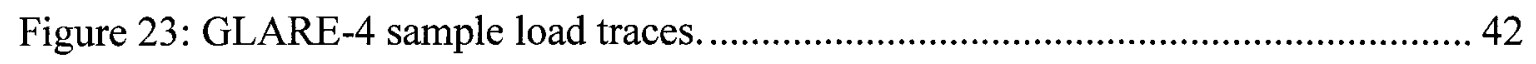

Figure 24: Strain gauge locations for four-gauge (a) and three-gauge (b) configurations.

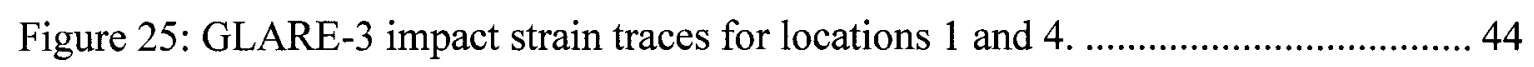

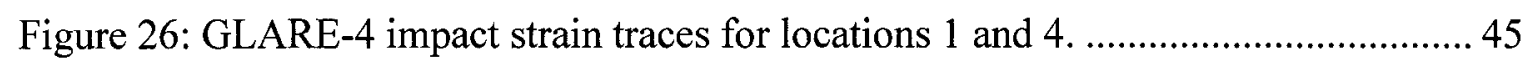

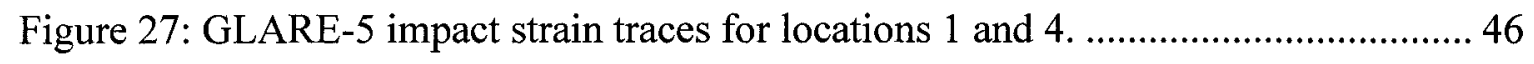

Figure 28: Penetrant enhanced x-ray images of impact damage. ................................. 47

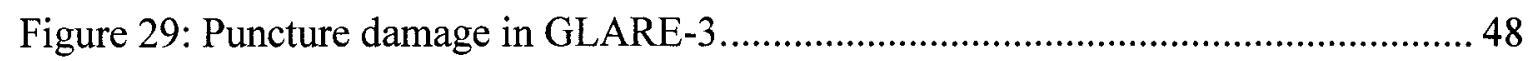

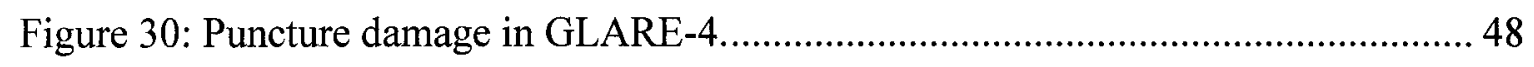

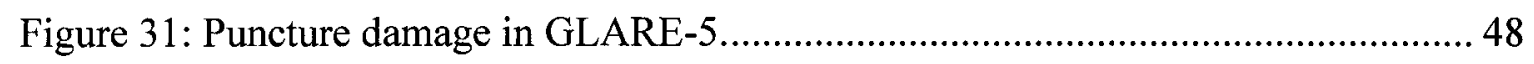

Figure 32: Cross-section of an unimpacted GLARE-3 specimen............................... 49

Figure 33: Cross-section of an unimpacted GLARE-4 specimen............................... 49

Figure 34: Cross-section of an unimpacted GLARE-5 specimen................................. 49

Figure 35: Sample GLARE-3-31 (15.73 J) (a) x-ray and (b) cross-section..................... 51

Figure 36: GLARE-3-31 (15.73 J) fibre delamination. ........................................... 51

Figure 37: GLARE-3-33 (36.42 J) (a) x-ray and (b) cross-section............................... 52

Figure 38: Matrix cracking in sample GLARE-3-33 in the $90^{\circ}$ layer............................. 52

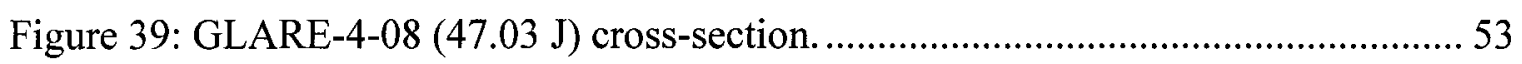

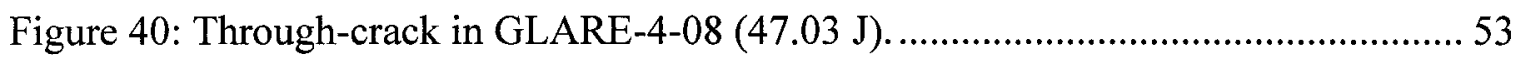

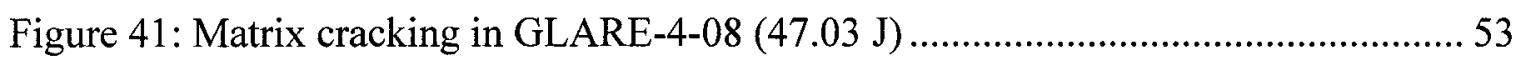


Figure 42: GLARE-5-04 (55 J) (a) x-ray and (b) cross-section.

Figure 43: Delamination and fibre-bridging in GLARE-5-04 on (a) left and (b) right of

dent...... 54

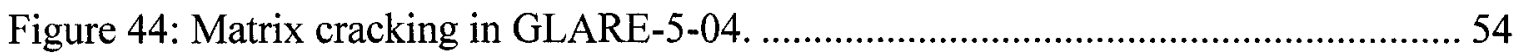

Figure 45: GLARE-5-12 (35 J) (a) x-ray and (b) cross-section....................................... 55

Figure 46: GLARE-5-12 matrix cracking and top $90^{\circ}$ layer adhesive failure.................. 55

Figure 47: A basic FML splice concept (not to scale)......................................................... 60

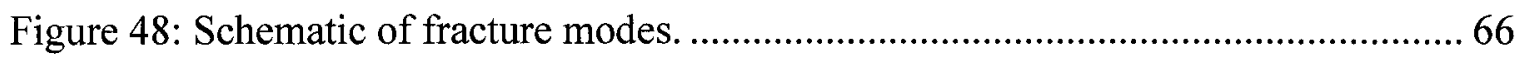

Figure 49: Required components of the FML damage modelling methodology.............. 72

Figure 50: Modelling methodology components and task relationships. ......................... 73

Figure 51: Nodal locations for the LS-DYNA thick-shell element. ................................ 80

Figure 52: Manufacturer's stress-strain data for aluminum 2024-T3 sheet...................... 88

Figure 53: Basic impact geometry for the preliminary impact simulations...................... 89

Figure 54: Edge-seeded "rough" mesh. ........................................................................ 91

Figure 55: Basic mesh used for "low," "medium" and "high" mesh density simulations.91

Figure 56: Absorbed energy versus impact energy for aluminum low-velocity impact experiments and simulations.

Figure 57: Peak force versus impact energy for aluminum low-velocity impact experiments and simulations. 94

Figure 58: Dent depth versus impact energy for aluminum low-velocity impact

experiments and simulations. 94

Figure 59: CPU time versus number of elements for preliminary simulations. 95

Figure 60: Half-symmetry mesh geometry. 97 
Figure 61: Impactor model geometry and mesh.

Figure 62: Stress-strain curves for 2024-T3 from various sources............................. 99

Figure 63: Impact energy results from material sensitivity study.............................. 101

Figure 64: Peak impact force from material sensitivity study. ................................. 101

Figure 65: Dent depth results from material property sensitivity study. ..................... 102

Figure 66: Absorbed Energy versus Impact Energy for the final impact simulations.... 103

Figure 67: Maximum Force versus Impact Energy for the final simulations. ................ 104

Figure 68: Permanent Deflection versus Impact Energy for the final impact simulations.

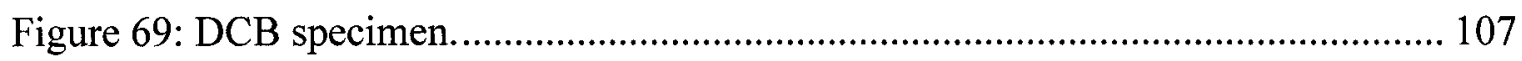

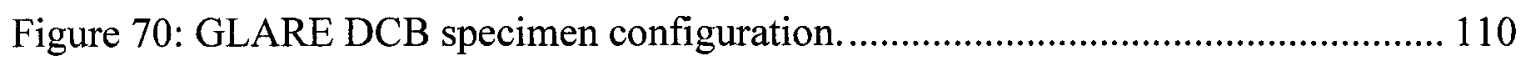

Figure 71: Acceptable load curve from a GLARE-5 DCB test. ................................. 111

Figure 72: Unacceptable load curve from a GLARE-3 DCB test................................ 111

Figure 73: Optical micrograph of the failure surface in a typical DCB specimen......... 112

Figure 74: (a) Fibre bridging in a typical FML DCB specimen and (b) in an impacted

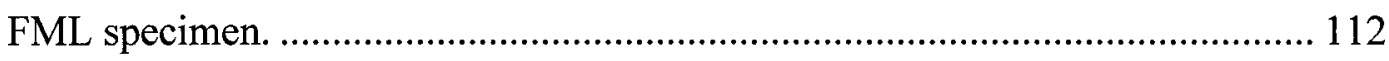

Figure 75: Representation of the micro-meso scale definition of damage (adapted from

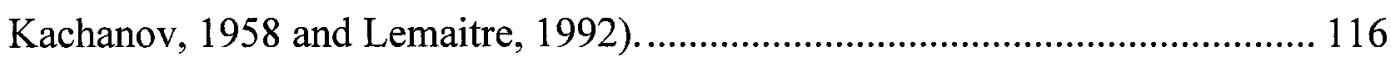

Figure 76: Prepreg elastic response verification tensile coupon (half-geometry). ........ 130

Figure 77: Comparison of simulated stress-strain response for prepreg coupon using the orthotropic-elastic model and the UMAT without damage. ........................... 131

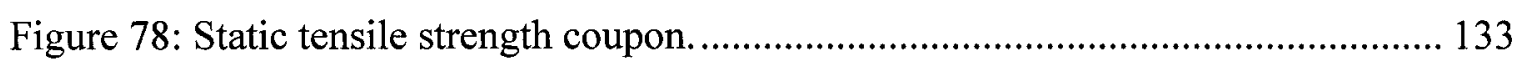

Figure 79: Typical GLARE-3-3/2 measured stress-strain curves for $0^{\circ}$ and $15^{\circ} \ldots \ldots \ldots 134$ 
Figure 80: Low-density mesh GLARE coupon geometry............................................ 135

Figure 81: High-density mesh GLARE coupon geometry........................................... 135

Figure 82: Effect of mesh density on the stress-strain response of the GLARE coupons.

Figure 83: Comparison between simulated stress-strain response and experimental data

for $0^{\circ}$ GLARE-3-3/2

Figure 84: Comparison between simulated response and experimental data for $15^{\circ}$

GLARE-3-3/2 loaded in tension.

Figure 85: Mesh geometry for GLARE impact simulations............................................ 143

Figure 86: Stress-strain curve for aluminum 2024-T3 ................................................. 144

Figure 87: Stress-displacement correction curve ......................................................... 146

Figure 88: Impact energy versus time for three different mesh densities of a GLARE-3 impact simulation.

Figure 89: Impact force versus time for three different mesh densities of a GLARE-3

impact simulation.

Figure 90: Dent depth versus time for three different mesh densities with GLARE-3. . 150

Figure 91: Predicted impacted panel geometries for (a) low-, (b) medium- and (c) high-

density meshes.

Figure 92: Typical absorbed energy versus time curves for simulations and experiments

with GLARE-3 impacted at $25 \mathrm{~J}$

Figure 93: Absorbed Energy versus Impact Energy for GLARE-3 ………………….... 154

Figure 94: Absorbed Energy versus Impact Energy for GLARE-4.............................. 154

Figure 95: Absorbed Energy versus Impact Energy for GLARE-5............................... 155 
Figure 96: Typical dent depth versus time plots for GLARE-3-2/1 impacted with 25 J.156

Figure 97: Dent Depth versus Impact Energy for GLARE-3 . ....................................... 157

Figure 98: Dent Depth versus Impact Energy for GLARE-4 ........................................ 157

Figure 99: Dent Depth versus Impact Energy for GLARE-5 ...................................... 158

Figure 100: Typical impact force vs. time plots for GLARE-3-2/1 impacted at 25 J. ... 159

Figure 101: Maximum Force vs. Impact Energy for GLARE-3 ..................................... 160

Figure 102: Maximum Force vs. Impact Energy for GLARE-4 ................................... 160

Figure 103: Maximum Force vs. Impact Energy for GLARE-5 ...................................... 161

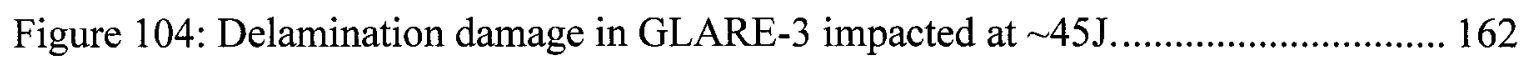

Figure 105: Typical delamination damage predicted using the (a) tied-interface, (b) simple-tiebreak interface, (c) corrected-tiebreak interface and (d) UMAT/simple-

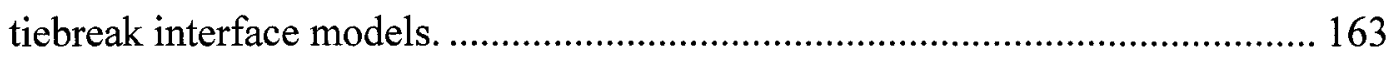

Figure 106: Damage maps of GLARE-3 impacted at $25 \mathrm{~J}$ from experiment, simpletiebreak interface, corrected-tiebreak interface and UMAT simulations............. 165

Figure 107: Damage maps of GLARE-3 impacted at $35 \mathrm{~J}$ from experiment, simpletiebreak interface, corrected-tiebreak interface and UMAT simulations............. 165

Figure 108: Damage maps of GLARE-4 impacted at $35 \mathrm{~J}$ from experiment, simpletiebreak interface and UMAT simulations......................................................... 166

Figure 109: Damage maps of GLARE-4 impacted at $45 \mathrm{~J}$ from experiment, simpletiebreak interface and UMAT simulations 166

Figure 110: Damage maps of GLARE-4 impacted at $55 \mathrm{~J}$ from experiment, simpletiebreak interface and UMAT simulations. 167 
Figure 111: Damage maps of GLARE-5 impacted at $35 \mathrm{~J}$ for experiment and UMAT simulations.

Figure 112: Damage maps of GLARE-5 impacted at $45 \mathrm{~J}$ for experiment and UMAT simulations. 168

Figure 113: Damage maps of GLARE-5 impacted at $55 \mathrm{~J}$ for experiment and UMAT simulations. 168

Figure 114: Percent difference between GLARE-3 absorbed energy predictions and experimental results.

Figure 115: Percent difference between GLARE-3 permanent dent depth predictions and experimental results. 180

Figure 116: Percent difference between GLARE-3 peak impact force predictions and experimental results.

Figure C1: Absorbed energy for the NASA and NRC impact fixtures. 207

Figure C2: Maximum force versus impact energy for the NASA and NRC fixtures.... 207

Figure C3: Maximum force versus absorbed energy for the NASA and NRC impact fixtures. 209

Figure E1: Sample GLARE-3-31 (15.73 J) (a) x-ray and (b) cross-section. 213

Figure E2: GLARE-3-31 (15.73 J) fibre delamination. 213

Figure E3: GLARE-3-32 (26.19 J) (a) x-ray and (b) cross-section. 213

Figure E4: GLARE-3-33 (36.42 J) (a) x-ray and (b) cross-section. 214

Figure E5: Matrix cracking in 3-33 in the 90 o layer. 214

Figure E6: GLARE-3-34 (46.72 J) (a) x-ray and (b) cross-section. 214

Figure E7: Intralaminar cracking in GLARE-3-34 (46.72 J). 215 
Figure E8: (a) Delamination in GLARE-4-16 and (b) unaffected region..................... 215

Figure E9: GLARE-4-08 (47.03 J) cross-section................................................ 215

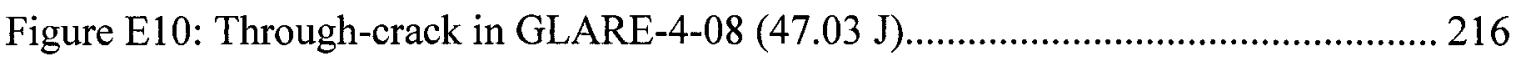

Figure E11: Matrix cracking in GLARE-4-08 (47.03 J)........................................ 216

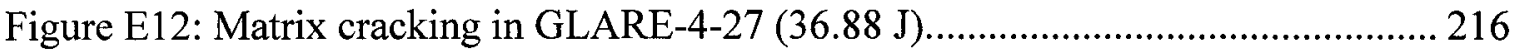

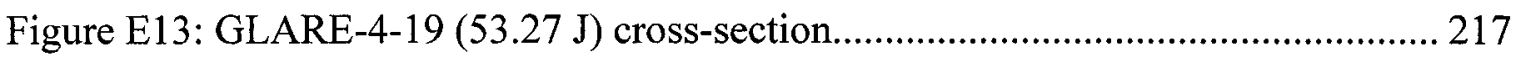

Figure E14: GLARE-4-19 (53.27 J) detail of cracked area. ...................................... 217

Figure E15: Matrix cracking and intralaminar failures in GLARE-4-19.................... 217

Figure E16: Delaminated regions in prepreg layers of GLARE-4-19. ...................... 218

Figure E17: GLARE-5-04 (55 J) (a) x-ray and (b) cross-section. ............................. 218

Figure E18: Delamination and fibre-bridging in GLARE-5-04.............................. 218

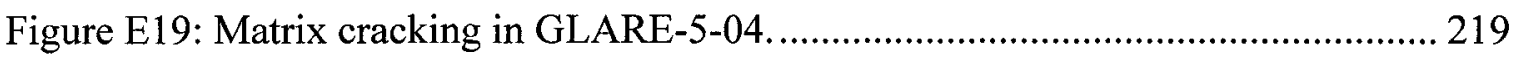

Figure E20: GLARE-5-11 (25 J) (a) x-ray and (b) cross-section. .............................. 219

Figure E21: GLARE-5-11 showing delamination damage....................................... 219

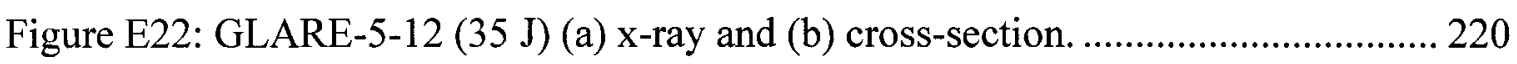

Figure E23: GLARE-5-12 matrix cracking and top $90^{\circ}$ layer adhesive failure............. 220 


\section{NOMENCLATURE}

\section{GLARE and ARALL designation used throughout this thesis:}

FML-X-Y/Z-( $\left.\mathbf{t}_{\mathfrak{a}}\right)$ where:

FML - a fibre-metal-laminate (e.g. GLARE, ARALL, etc)

X - type designation for GLARE or ARALL (e.g. GLARE-2)

$\mathrm{Y}$ - number of alloy layers

$Z$ - number of prepreg layers

$\mathrm{t}_{\mathrm{a}}$ - thickness of alloy sheets in $\mathrm{mm}$ (commonly omitted)

\section{Abbreviations:}

AEI Aviation Equipment Industries

AFRP Aramid Fibre Reinforced Polymer

ALLIC ALuminum LIthium Carbon reinforced laminate

ARALL Aramid Reinforced ALuminum Laminate

BRALL Bamboo Reinforced ALuminum Laminate

BVID Barely Visible Impact Damage

CARALL CArbon Reinforced ALuminum Laminate (also CARE)

CARE CArbon REinforced laminate (also CARALL)

CDM Continuum Damage Mechanics

CFRP Carbon Fibre Reinforced Polymer

DCB Double Cantilever Beam 
DCLS Double Crack Lap Shear

FAA Federal Aviation Administration

FAR Federal Airworthiness Regulation

FML Fibre-Metal-Laminate

GFRP Glass Fibre Reinforced Polymer

GLARE GLAss REinforced aluminum laminate

HTCL Hybrid Titanium Carbon Laminate (see also TiGr)

PEEK PolyEther Ether Ketone

SEN Single Edge Notched specimen

SLC Structural Laminates Corporation

SLI Structural Laminates Industries

TiGr Titanium-graphite fibre-metal-laminates (see also HTCL)

TUDelft Technical University of Delft

UD Uni-directional

\section{Latin Symbols:}

$\begin{array}{ll}a & \text { Distance from centre of panel, crack length } \\ a_{i j k l} & \text { Fourth order elasticity stiffness tensor } \\ a_{0} & \text { Initial crack length } \\ b & \text { Cantilever beam width, damage coupling parameter } \\ d, d, & \text { Damage coefficients } \\ d_{i} & \text { Deflection of coupon } \\ d_{i, d_{i-1}} & \text { Deflection of plate at time i and i-1, respectively }\end{array}$




\begin{tabular}{|c|c|}
\hline$g$ & Gravitational constant \\
\hline$h$ & Half beam thickness, enthalpy \\
\hline$k$ & Contact rigidity \\
\hline$m$ & Mass of the crosshead \\
\hline$n$ & Power index \\
\hline$q$ & Power index \\
\hline$r$ & Radius of panel \\
\hline$s$ & Strength limit of a material \\
\hline$t$ & Thickness, time \\
\hline$t_{a l}$ & Aluminum layer thickness \\
\hline$\Delta t$ & Time increment \\
\hline$u$ & In-plane deflection \\
\hline$w$ & General deflection in z-direction \\
\hline$w_{o}$ & Deflection of centre of panel in z-direction \\
\hline$A_{i j}$ & Laminate in-plane stiffness matrix \\
\hline$C$ & Compliance matrix \\
\hline$D_{a}$ & Empirical constant for delamination initiation \\
\hline$\left(E_{o}\right)_{i}$ & Absorbed energy at time $i$ \\
\hline$E_{11}$ & Local 11 elastic modulus \\
\hline$E_{22}$ & Local 22 elastic modulus \\
\hline$E_{e}$ & Elastic material strain energy \\
\hline$E_{D}$ & Damaged material strain energy \\
\hline$E_{f}$ & Prepreg modulus \\
\hline
\end{tabular}

xxiv 


\begin{tabular}{|c|c|}
\hline$E_{i m p}$ & Impact energy \\
\hline$E^{o}$ & Elastic (undamaged) Young's modulus \\
\hline$E_{x}$ & Global longitudinal elastic modulus \\
\hline$E_{y}$ & Global transverse elastic modulus \\
\hline$F$ & Contact force \\
\hline$G_{12}$ & Local 12 shear modulus \\
\hline$G_{I c}$ & $\begin{array}{l}\text { Critical fracture toughness/strain energy release rate for Mode I } \\
\text { delamination }\end{array}$ \\
\hline$G_{I I c}$ & $\begin{array}{l}\text { Critical fracture toughness/strain energy release rate for Mode II } \\
\text { delamination }\end{array}$ \\
\hline$G_{x y}$ & Shear modulus in $x y$ \\
\hline$J$ & Jacobian matrix \\
\hline$J_{2}$ & Von Mises equivalent stress \\
\hline$\Delta K_{e f f}$ & Effective stress intensity factor \\
\hline$L$ & Beam length \\
\hline$P$ & Applied load \\
\hline$P_{i}, P_{i-1}$ & Load at times $i$ and $i-1$, respectively \\
\hline$Q$ & Elasticity matrix \\
\hline$R$ & Ratio of maximum and minimum stress during fatigue cycling \\
\hline$S$ & Unloading rigidity \\
\hline$U$ & Energy (elastic) \\
\hline$V_{i}, V_{i-1}$ & Instantaneous velocity at time $i$ and $i-1$, respectively \\
\hline$\overline{V_{i}}$ & Average velocity at time $i$ \\
\hline
\end{tabular}


$Y \quad$ Damaged strain energy release rate

$Y_{o}, Y_{o} \quad$ Damage development law variables

$Y_{c}, Y_{c}{ }^{\prime} \quad$ Damage development law variables

$Y_{r}, Y_{s} \quad$ Damage development law variables

\section{Greek Symbols:}

$\begin{array}{ll}\alpha & \text { Indentation depth } \\ \alpha_{m} & \text { Indentation depth corresponding to start of impact unloading } \\ \alpha_{p} & \text { Critical indentation depth } \\ \alpha_{o} & \text { Permanent indentation depth } \\ \gamma_{r} & \text { In-plane shear strain } \\ \delta, \partial & \text { Differential operators } \\ \varepsilon_{f} & \text { Fibre strain } \\ \varepsilon_{r} & \text { Radial strain } \\ \varepsilon_{\theta} & \text { Circumferential strain } \\ \zeta & \text { Parametric z co-ordinate } \\ \rho_{y} & \text { Noreal density } \\ \eta & \text { Fibre angle, thermodynamic temperature } \\ \rho_{y} & \text { Density }\end{array}$

$\sigma_{1}, \sigma_{13}, \sigma_{2}, \sigma_{23} \quad$ Normal and shear stresses in local co-ordinate directions 


$\begin{array}{ll}\sigma_{12}^{r} & \text { Shear rupture stress } \\ v & \text { Poisson's ratio } \\ v_{12} & \text { Local longitudinal Poisson's ratio } \\ v_{2 l} & \text { Local transverse Poisson's ratio } \\ v_{x y} & \text { Global longitudinal Poisson's ratio } \\ v_{y x} & \text { Global transverse Poisson's ratio } \\ \phi & \text { Plasticity threshold, shape functions } \\ \xi & \text { Normalized deflection }\left(w / w_{o}\right), \text { parametric } \mathrm{x} \text { co-ordinate } \\ \psi & \text { Free energy density }\end{array}$




\section{CHAPTER 1}

\section{INTRODUCTION}

Fibre-metal-laminates (FMLs) are a new class of hybrid materials consisting of thin metallic layers laminated with fibre-reinforced polymer (FRP) layers (Figure 1). The resulting laminates possess lower density, higher strength and improved fatigue resistance as compared to traditional monolithic aircraft alloys. These laminates can be produced with a wide variety of alloys and prepregs (fibres that are pre-impregnated with resin prior to being cured). This thesis presents the results of an extensive impact testing program. A methodology for modelling the impact damage in these laminates is also proposed and applied to examine the impact response of FML panels.

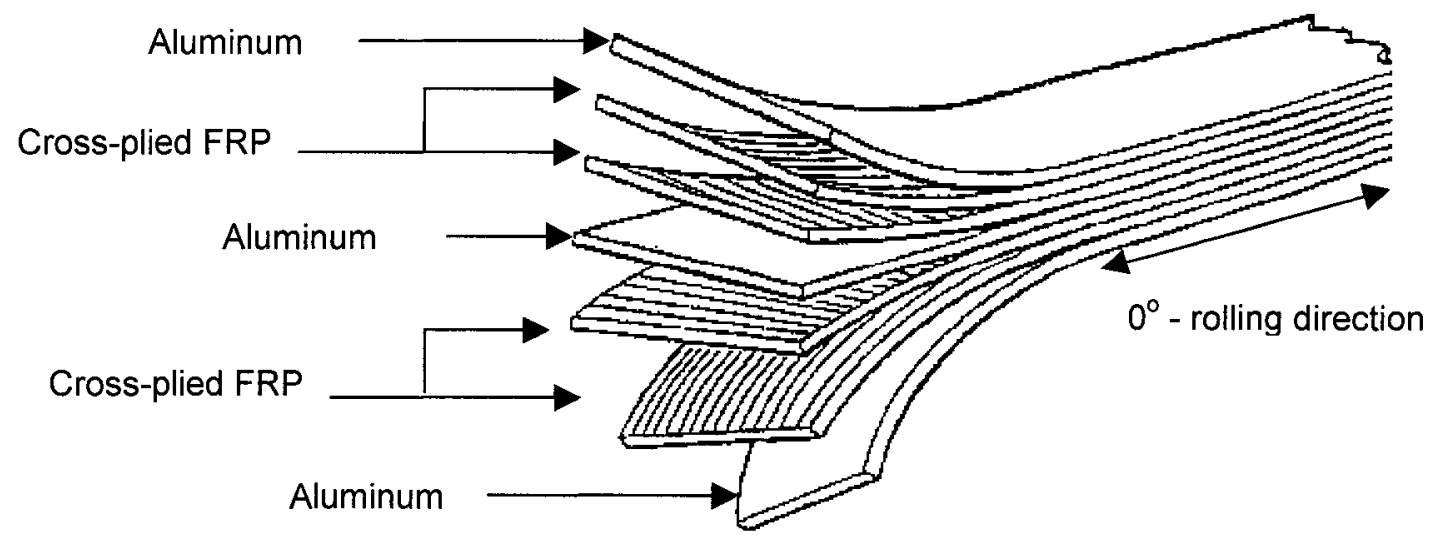

Figure 1: Basic FML configuration (adapted from Asundi and Choi, 1997). 


\subsection{Development of FML Technology}

One of the critical damage modes in modern metallic aircraft structures is that of fatigue crack growth. A tremendous amount of research has been devoted to the study of this damage mode since the crashes of de Havilland Comet jetliners caused by metal fatigue in the 1950s. More recently, the Aloha Airlines near-disaster caused a renewed emphasis to be placed on the study of fatigue damage in riveted metallic structures. A significant amount of research has been devoted to developing damage tolerant materials that may be applied to aircraft structures to combat this costly problem.

As new materials are developed there is a constant struggle between the desire to reduce weight and the necessity of ensuring passenger safety. Traditional composite materials have been applied to aircraft structures in an attempt to address both of these issues. These materials provided reduced density and improved fatigue damage tolerance over metallic materials. In many cases, traditional composites also provided increased stiffness- and strength-to-weight ratios. However, there remain significant barriers to the widespread application of these materials in aircraft structures. For example, composite materials are sensitive to low-velocity impacts. Impact damage in composites is often widespread within the laminate but there may be little surface evidence of damage, this is referred to as "barely visible impact damage" or BVID.

Even as composite materials were being developed, research continued into techniques for improving the fatigue crack growth resistance of metallic materials. Some of this work resulted in the development of improved alloy formulations or heat treatments. Mechanical methods for improving the crack growth resistance were also investigated. Early research demonstrated, that by adhesively laminating sheets of metal 
together, significant increases in the fracture toughness were possible (Kaufman, 1967, Bijlmer, 1978 and Johnson, 1983).

Kaufman observed that the fracture toughness of laminated plates of 7075-T6 made up of eight $1.6 \mathrm{~mm}$ thick layers, was double that of monolithic plates of the same total thickness as shown in Figure 2. The laminated plates possess critical stress-intensity factors that approach those of the constituent monolithic layers. In thin materials the amount of plastic constraint at the crack tip is smaller and plane-stress conditions are dominant (Hertzberg, 1996). Therefore, the thin sheets comprising the laminated plates exhibit increased toughness. Damage tolerance of the laminated plates is improved because of lower stress-intensity factors in cracked layers due to stress transfer to undamaged layers and high resistance to inter-ply crack transfer (Johnson, 1983).

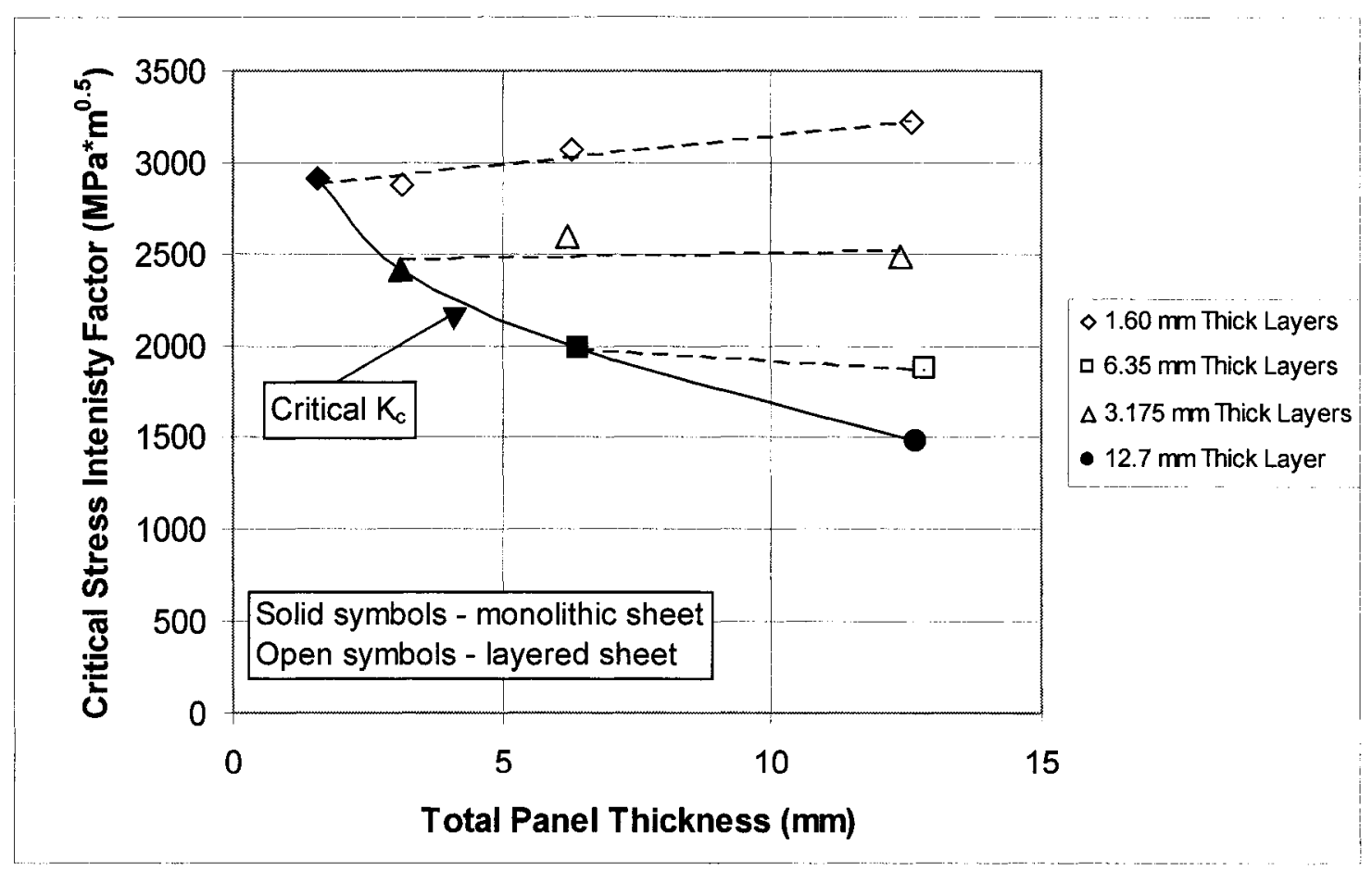

Figure 2: Variation in critical stress-intensity factors for layered and monolithic sheets (adapted from Kaufman, 1967). 
Fatigue crack growth resistance was improved further by including fibrereinforced prepreg layers between the metal layers to produce fibre-metal-laminates. Unbroken fibres bridge the crack tip and slow its growth (Bijlmer, 1978). Early FML variants developed at the Technical University of Delft (TUDelft) in the Netherlands consisted of unidirectional aramid prepreg layers laminated with 2024-T3 or 7075-T6 aluminum sheets (Marissen and Vogelsang, 1981, Vogelsang et al., 1981, Bucci et al., 1989 and Gregory and Roebroeks, 1991). Aramid reinforced polymer was selected due to its high blunt-notch strength. These Aramid Reinforced Aluminum Laminates (ARALL) are only manufactured in a unidirectional variant. Carbon and E-glass fibres, among others, were eventually used as well.

Currently, S2-glass based FMLs are available commercially under the trade name GLARE aluminum laminates. Structural Laminates Corporation (SLC) manufactures these products in Europe through a licensing agreement with Fokker Aerostructures, and Aviation Equipment Industries (AEI) manufactures them in North America through a similar agreement. Another company, MCGill Corp., manufactures woven glass and aramid FML panels for use as cargo bay liners. Comtek Ltd., a Canadian composite materials company, is currently installing these laminates as a retrofit into certain types of regional aircraft. FMLs using carbon fibres with aluminum-lithium (ALLIC), graphite fibres with titanium (TiGr) and bamboo or vinylon fibres with aluminum are also being developed.

Fibre-metal-laminates incorporate some of the benefits of traditional composites, such as reduced density and fatigue damage tolerance. Many of the desirable properties of monolithic metals including formability and plastic deformation prior to failure are 
also retained by FMLs. The combination of metal and prepreg layers also provides crack growth resistance, impact damage resistance and good impact damage visibility due to formation of plastically deformed dents. This material system seems to meet the requirements of improving passenger safety while decreasing the weight of the aircraft. However, as with any new material, a detailed examination of the properties of FMLs is required before selecting them for an aircraft design. 


\subsection{Mechanical Properties of Typical FMLs}

Table 1 summarizes the composition of ARALL, GLARE and two woven fibremetal-laminates. The manufacturers' names are shown in parentheses under the commercial designation of the FML. In addition to the resins quoted in the table, newer varieties of GLARE are available which use FM94 resin from Cytec instead of the usual F-185 from Hexcel or the older AF-163-2 from 3M.

Typical mechanical properties of several FMLs can be found in Table 2. In this table, A refers to ARALL, G refers to GLARE, $L$ is the longitudinal direction parallel to the rolling direction of the aluminum $\left(0^{\circ}\right)$ and LT is the longitudinal-transverse $\left(90^{\circ}\right)$ direction perpendicular to the rolling direction of the aluminum. As can be seen from the data, GLARE typically has a higher strength than monolithic aluminum. The lower stiffness of GLARE must be considered when replacing aluminum in vibration prone areas of an aircraft. The density per unit area, or areal density, is also lower in GLARE laminates than monolithic aluminum. If GLARE laminates are used to replace equal thickness aluminum sheets in an aircraft, an immediate reduction in structural weight can be realized.

To improve the overall fatigue performance of the laminates, a plastic post-cure stretch is sometimes applied during the manufacturing process. The standard plastic strain of $0.4 \%$ reverses the as-cured stress-state in the laminae by inducing a compressive stress in the aluminum and a tensile stress in the prepreg. The compressive residual stress in the aluminum layers increases their resistance to fatigue crack initiation. None of the panels used in this project received this treatment. 
Table 1: Summary of some commercially available FMLs.

\begin{tabular}{|c|c|c|c|c|c|c|}
\hline \begin{tabular}{|l|} 
Commercial \\
Designation \\
\end{tabular} & $\begin{array}{c}\text { Basic } \\
\text { Description }\end{array}$ & $\begin{array}{c}\text { Metal Alloy } \\
\text { and Thickness }\end{array}$ & \begin{tabular}{|c|} 
Prepreg \\
Thickness \\
\end{tabular} & Fibre & Adhesive & \begin{tabular}{|c|} 
Post-cure \\
Stretch
\end{tabular} \\
\hline $\begin{array}{l}\text { ARALL-2 } \\
\text { (SLC) }\end{array}$ & $\begin{array}{l}120^{\circ} \mathrm{C} \text { cure } \\
\text { prepreg, } 50 \% \text { by } \\
\text { volume UD fibres }\end{array}$ & $\begin{array}{l}2024-\mathrm{T} 3, \mathrm{t}=0.2 \\
-0.4 \mathrm{~mm}\end{array}$ & $0.21 \mathrm{~mm}$ & Aramid & AF-163-2 & $\begin{array}{l}\text { with or } \\
\text { without } \\
0.4 \%\end{array}$ \\
\hline $\begin{array}{l}\text { ARALL-3 } \\
\text { (SLC) }\end{array}$ & $\begin{array}{l}120^{\circ} \mathrm{C} \text { cure } \\
\text { prepreg, } 50 \% \text { by } \\
\text { volume UD fibres }\end{array}$ & $\begin{array}{l}7475-\mathrm{T} 76, \mathrm{t}=0.3 \\
-0.4 \mathrm{~mm}\end{array}$ & $0.21 \mathrm{~mm}$ & Aramid & AF-163-2 & $0.4 \%$ \\
\hline $\begin{array}{l}\text { ARALL-4 } \\
\text { (SLC) }\end{array}$ & $\begin{array}{l}175^{\circ} \mathrm{C} \text { cure } \\
\text { prepreg, } 50 \% \text { by } \\
\text { volume UD fibres }\end{array}$ & $\begin{array}{l}2024-\mathrm{T} 8, \mathrm{t}=0.3- \\
0.4 \mathrm{~mm}\end{array}$ & $0.21 \mathrm{~mm}$ & Aramid & AF-191 & $\begin{array}{l}\text { with or } \\
\text { without } \\
0.4 \%\end{array}$ \\
\hline $\begin{array}{l}\text { GLARE-1 } \\
\text { (SLC) }\end{array}$ & $\begin{array}{l}120^{\circ} \mathrm{C} \text { cure } \\
\text { prepreg, } 60 \% \text { by } \\
\text { volume UD fibres }\end{array}$ & $\begin{array}{l}7475-\mathrm{T} 6, \mathrm{t}=0.3 \\
\mathrm{~mm}\end{array}$ & $0.25 \mathrm{~mm}$ & S2-glass & $F-185$ & $0.3-0.5 \%$ \\
\hline $\begin{array}{l}\text { GLARE-2 } \\
\text { (SLC) }\end{array}$ & $\begin{array}{l}120^{\circ} \mathrm{C} \text { cure } \\
\text { prepreg, } 60 \% \text { by } \\
\text { volume UD fibres }\end{array}$ & $\begin{array}{l}2024-\mathrm{T} 3, \mathrm{t}=0.2 \\
-0.4 \mathrm{~mm}\end{array}$ & $0.25 \mathrm{~mm}$ & S2-glass & F-185 & $\begin{array}{l}\text { no pre- } \\
\text { strain }\end{array}$ \\
\hline $\begin{array}{l}\text { GLARE-3 } \\
\text { (SLC) }\end{array}$ & $\begin{array}{l}120^{\circ} \mathrm{C} \text { cure } \\
\text { prepreg, } 60 \% \text { by } \\
\text { volume } 50 / 50 \\
\text { cross ply fibres }\end{array}$ & $\begin{array}{l}2024-\mathrm{T} 3, \mathrm{t}=0.2 \\
-0.4 \mathrm{~mm}\end{array}$ & $0.25 \mathrm{~mm}$ & S2-glass & $F-185$ & $\begin{array}{l}\text { no pre- } \\
\text { strain }\end{array}$ \\
\hline $\begin{array}{l}\text { GLARE-4 } \\
\text { (SLC) }\end{array}$ & $\begin{array}{l}120^{\circ} \mathrm{C} \text { cure } \\
\text { prepreg, } 60 \% \text { by } \\
\text { volume fibres with } \\
70 \% \text { in rolling } \\
\text { direction }\end{array}$ & $\begin{array}{l}2024-\mathrm{T} 3, \mathrm{t}=0.2 \\
-0.4 \mathrm{~mm}\end{array}$ & $0.375 \mathrm{~mm}$ & S2-glass & $\mathrm{F}-185$ & $\begin{array}{l}\text { no pre- } \\
\text { strain }\end{array}$ \\
\hline $\begin{array}{l}\text { GLARE-5 } \\
\text { (SLC) }\end{array}$ & $\begin{array}{l}120^{\circ} \mathrm{C} \text { cure } \\
\text { prepreg, } 60 \% \text { by } \\
\text { volume } 50 / 50 \\
\text { cross ply fibres }\end{array}$ & $\begin{array}{l}2024-\mathrm{T} 3, \mathrm{t}=0.4 \\
-0.51 \mathrm{~mm}\end{array}$ & $0.51 \mathrm{~mm}$ & S2-glass & $F-185$ & $\begin{array}{l}\text { no pre- } \\
\text { strain }\end{array}$ \\
\hline $\begin{array}{l}\text { GILLFLOOR } \\
5033 \text { (MCGill) }\end{array}$ & Woven aramid & 2024-T3 & & $\begin{array}{l}\text { Woven } \\
\text { aramid }\end{array}$ & & $\begin{array}{l}\text { no pre- } \\
\text { strain }\end{array}$ \\
\hline $\begin{array}{l}\text { GILLFLOOR } \\
5433 \text { (MCGill) }\end{array}$ & Woven glass & 2024-T3 & & $\begin{array}{l}\text { Woven } \\
\text { S-glass }\end{array}$ & & $\begin{array}{l}\text { no pre- } \\
\text { strain }\end{array}$ \\
\hline
\end{tabular}


Table 2: Summary of properties of several FMLs and 2024-T3 aluminum (Asundi and Choi, 1997 and Department of Defense, 1998).

\begin{tabular}{|c|c|c|c|c|c|c|c|c|}
\hline \multirow{2}{*}{ Parameter } & \multirow{2}{*}{ Direction } & \multicolumn{7}{|c|}{ Material (A - ARALL, G - GLARE) } \\
\hline & & 2024-T3 & A-2 & A-3 & G-2 & G-3 & G-4 & G-5 \\
\hline \multirow{2}{*}{$\begin{array}{l}\text { Ultimate tensile strength } \\
(\mathrm{MPa})\end{array}$} & $\mathrm{L}$ & 441 & 717 & 821 & 1213 & 745 & 1027 & 683 \\
\hline & LT & 434 & 317 & 780 & 317 & 745 & 607 & 683 \\
\hline \multirow{2}{*}{$\begin{array}{l}0.2 \% \text { Offset yield strength } \\
(\mathrm{MPa})\end{array}$} & $\mathrm{L}$ & 324 & 366 & 607 & 393 & 303 & 352 & - \\
\hline & LT & 290 & 228 & 331 & 228 & 303 & 25 & - \\
\hline \multirow{2}{*}{$\begin{array}{l}\text { Tensile elastic modulus } \\
(\mathrm{GPa})\end{array}$} & $\mathrm{L}$ & 72.4 & 66 & 66 & 66 & 58 & 57 & 70 \\
\hline & LT & - & 53 & 49 & 50 & 58 & 50 & 70 \\
\hline \multirow{2}{*}{ Ultimate strain (\%) } & $\mathrm{L}$ & 15.0 & 2.5 & 2.2 & 4.7 & 4.7 & 4.7 & - \\
\hline & LT & - & 12.7 & 8.6 & 10.8 & 4.7 & 4.7 & - \\
\hline \multirow{2}{*}{$\begin{array}{l}0.2 \% \text { offset compressive } \\
\text { yield strength (MPa) }\end{array}$} & $\mathrm{L}$ & 269 & 255 & 345 & 413 & 310 & 365 & 283 \\
\hline & LT & 310 & 234 & 360 & 236 & 310 & 285 & 283 \\
\hline \multirow{2}{*}{$\begin{array}{l}\text { Compressive elastic } \\
\text { modulus (GPa) }\end{array}$} & $L$ & 73.8 & 65 & 64 & 67 & 59 & 60 & - \\
\hline & LT & - & 53 & 50 & 52 & 59 & 54 & - \\
\hline \multirow{2}{*}{$\begin{array}{l}0.2 \% \text { offset shear yield } \\
\text { strength (MPa) } \\
\end{array}$} & $L-L T$ & - & 111 & 117 & - & - & - & - \\
\hline & LT-L & - & 111 & 117 & - & - & - & - \\
\hline \multirow{2}{*}{ Shear modulus (GPa) } & L-LT & 27.6 & 17 & 17 & - & - & - & - \\
\hline & LT-L & - & 16 & 16 & - & - & - & - \\
\hline Areal density $\left(\mathrm{kg} / \mathrm{m}^{2}\right)$ & $\mathrm{N} / \mathrm{A}$ & 2.82 & & & & 2.17 & 2.42 & 2.69 \\
\hline
\end{tabular}

Another benefit of using FMLs to replace monolithic aluminum in airframe structures is the increase in fatigue life. Figure 3 is a comparison of fatigue crack propagation in GLARE and aluminum riveted lap joints. The graph shows the aggregate length of all of the cracks growing from rivet holes in a lap joint fatigue specimen. Both specimens are subjected to the same load and frequency and use the same rivets. The crack lengths were normalized to zero to simplify comparison of the data. As can be seen, cracks in the GLARE specimen grew at a steady rate. However, in the aluminum specimen, the crack growth accelerated sharply before failure. Inspection intervals for monolithic airframes must be short enough to find such damage before it reaches the stage of rapid growth to failure. Inspection intervals for GLARE structures can be relaxed since crack growth does not proceed as rapidly. 


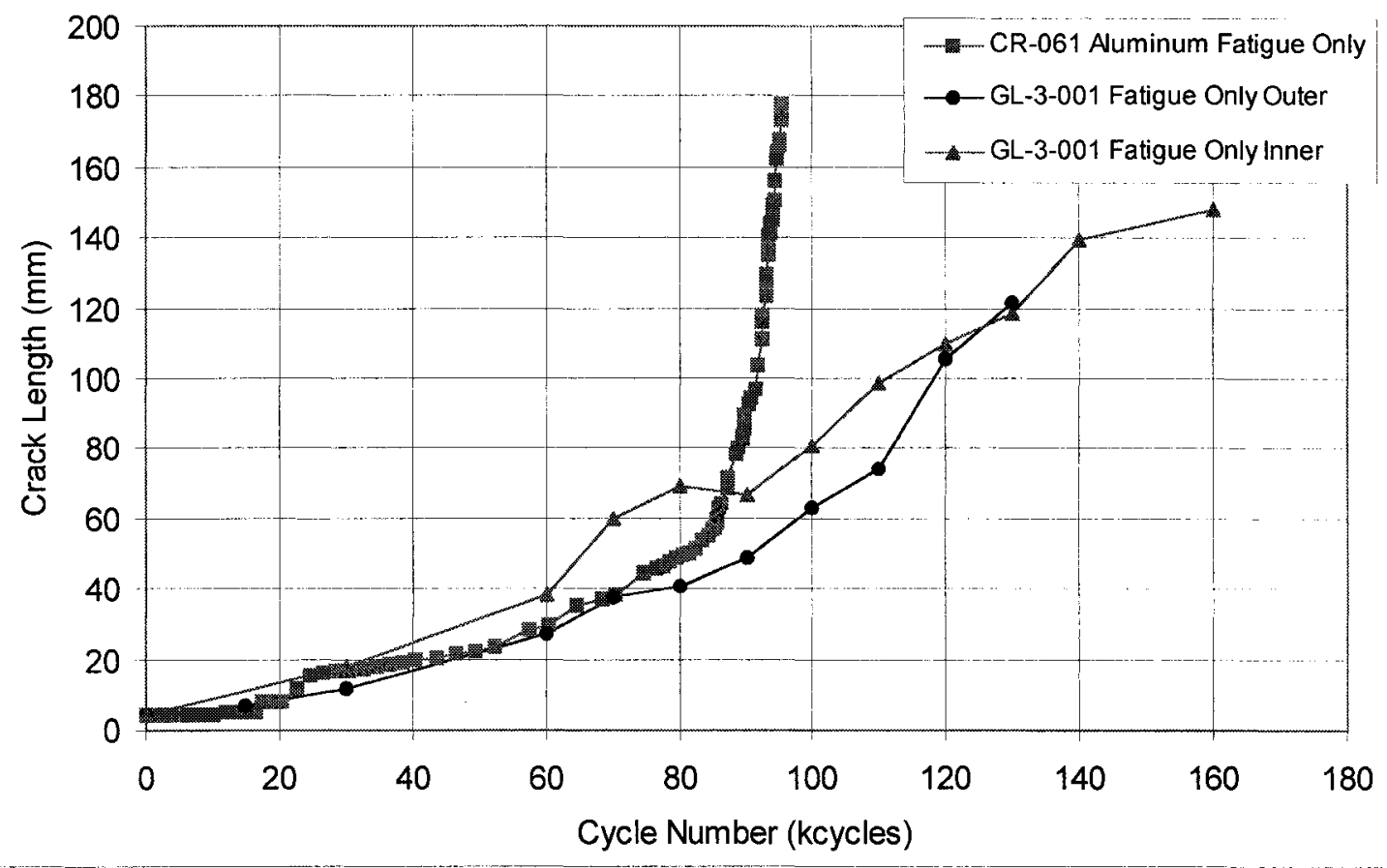

Figure 3: Aggregate multi-site fatigue crack propagation in GLARE-3-2/1 compared to monolithic aluminum (Laliberté et al., 1999e) [crack lengths were normalized to zero cycles]. 


\subsection{The FML Durability Project}

The FML Durability Project, a multi-year collaborative research program, was established between Carleton University, Bombardier Aerospace and the Institute for Aerospace Research (IAR-SMPL) of the National Research Council Canada (NRC) to examine the effect of various types of loading on GLARE FMLs.

Phase I (1998-1999) examined the impact damage tolerance, post-impact strength, multi-site fatigue damage and burn-through resistance of GLARE. Some of the results of the impact experiments have been published elsewhere (Laliberté, et al., 1999a, 1999b, 1999c, 1999d, 1999e, 2000a, 2001a, 2002b). Important results from these tests will be highlighted in the following paragraphs.

Tension-after-impact (TAI) tests were conducted on GLARE-3-2/1, GLARE-42/1, GLARE-5-2/1 and aluminum samples (Laliberté et al., 1999e). The test method was based upon a procedure described by Mattousch, 1993. The results showed a small decrease in the static strength of GLARE-3 and GLARE-5 following impact. However, the GLARE-4 coupons maintained the greatest fraction of the undamaged static strength. Recall from Section 1.2 that GLARE-4 has more fibres in the loading direction $\left(0^{\circ}\right)$.

Another property of interest to designers and certification authorities is that of post-impact fatigue damage growth. Two sets of post-impact fatigue tests were conducted as part of the FML Project (Laliberté et al., 1999e, 2000b, 2002a). In the first set of tests, impacted panels of GLARE-3-2/1, GLARE-4-2/1, GLARE-5-2/1 and aluminum were subjected to tension-tension fatigue. Cracks were observed to initiate at the edges of the impact induced dent. As in the TAI tests, the GLARE-4-2/1 specimens 
showed the highest resistance to the growth of fatigue cracks due to the higher amount of fibres in the loading direction. The second, more specific series of tests, examined offaxis post-impact fatigue crack growth in GLARE-4-3/2 laminates. These results showed that fatigue cracks in impacted FML panels manifested first on the front (impacted) face of the laminate. This observation is very significant; it means that in practice, fatigue cracks resulting from impact dents on FML skins would be easily detectable. Also, hidden fatigue cracks on the back (unimpacted) face lagged behind the development of damage on the front face. Figure 4 shows sample crack growth curves from the testing conducted at IAR-SMPL. The aggregate crack growth for the front and back faces of the specimen was measured using a travelling microscope. When the cracks on the front face reached the edge of the specimen, growth stopped and the cracks in the back face began to grow.

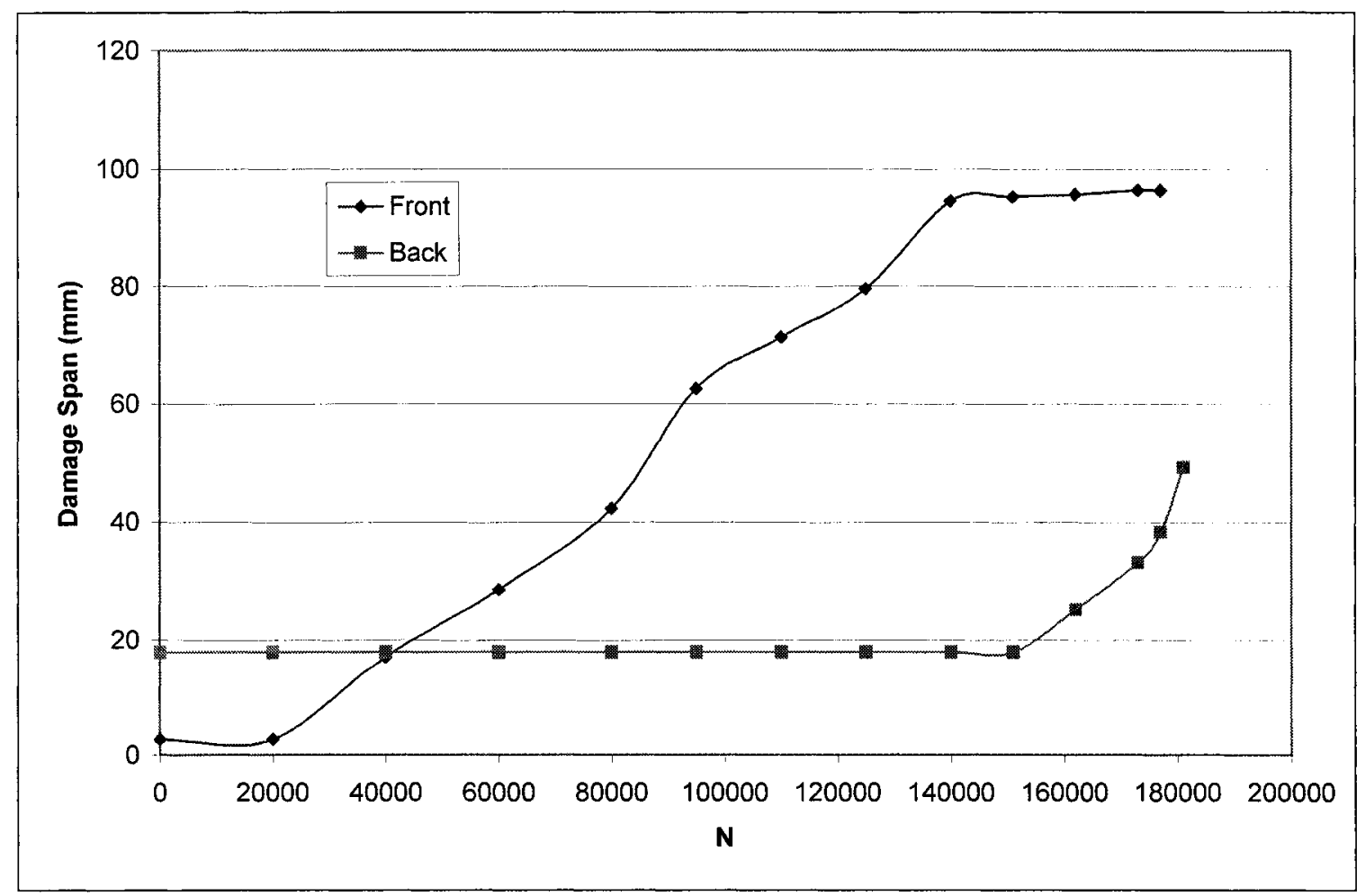

Figure 4: Sample post-impact fatigue results (Laliberté, et al., 2002) 
The final series of post-impact experiments measured the effect of impact damage on the shear-buckling behaviour of GLARE panels (Laliberté et al., 1999e and 2001b). Impacted specimens were installed in a "picture-frame" fixture that introduced a shear load along the specimen edges. Strain gauges were used to detect the onset of buckling in the panels. The buckling experiments built upon earlier work on the buckling of undamaged FML panels at IAR-SMPL.

Multi-site fatigue crack growth was also evaluated during Phase I. This damage mode occurs because the stresses on rivets in a lap-splice are approximately equal along the joint. Cracking will tend to initiate at the same time at each of the rivets. The cracks grow simultaneously until they reach a critical aggregate length and the entire structure fails catastrophically as happened to an Aloha Airlines Boeing 737-200 aircraft in 1988 . This problem is worsened by corrosion damage, which is still prevalent in modern aircraft. As part of the FML Durability Project, several simulated fuselage lap-splices were constructed using GLARE-3-2/1 (Laliberté et al. 1999e). The results showed that MSD damage in GLARE splices grew at a steady rate. In aluminum splices, the crack growth rates increased sharply near the end of the test. Also, when the aluminum layers in the GLARE splices had failed, the prepreg layers continued to carry the fatigue loads.

Phase II (2000-2003) consisted of investigations of off-axis fatigue, off-axis postimpact fatigue, static lap joint strength, bonded splice fatigue, and the buckling and postbuckling of large stiffened panels and cylinders (Jodoin et al., 2001a, 2001b, 2002; Shi and Poon, 2001). Tests of the delamination response and static tensile strength of GLARE were also performed during the first two years of Phase II. 


\subsection{Thesis Motivation}

Fibre-metal-laminates will be employed in next-generation civilian and military aircraft. For example, GLARE (GLAss REinforced) aluminum laminates have been selected as a fuselage material for the new Airbus A380 transport aircraft and TiGr (pronounced "tiger") laminates of titanium and graphite reinforced polymer are being considered for parts of the F-35 Joint Strike Fighter. ARALL (Aramid Reinforced ALuminum Laminates) and GLARE are already being used in secondary airframe structures that are subjected to impact loads such as cargo bay liners and wing flaps. Other FMLs are being installed in Bombardier RJ and European ATR regional aircraft as cargo bay liner retrofits.

As shown in Figure 5, there are many areas of civil transports that can suffer impact damage. The impactor could vary in size and mass from a small stone to a galley service vehicle. The energy levels have a similar variability depending on the relative speeds of the aircraft and the impactor. Relatively low-energy impacts can introduce significant internal damage in traditional composites that is not immediately apparent to the naked eye as shown in Figure 6. In contrast, impact damage in monolithic alloys is clearly indicated by the formation of a plastic dent.

Impact testing remains important as new FMLs continue to replace monolithic aluminum in impact-sensitive primary airframe structures. Impact damage experiments are time consuming and expensive; a limited test program together with finite element simulations would reduce both the cost and time required to certify new FMLs for aircraft. 
Wing and empennage leading edges -

$$
\text { Hail and bird strike }
$$

\section{Engine nacelles -}

Hail and bird strike, blade separation

ground handling equipment

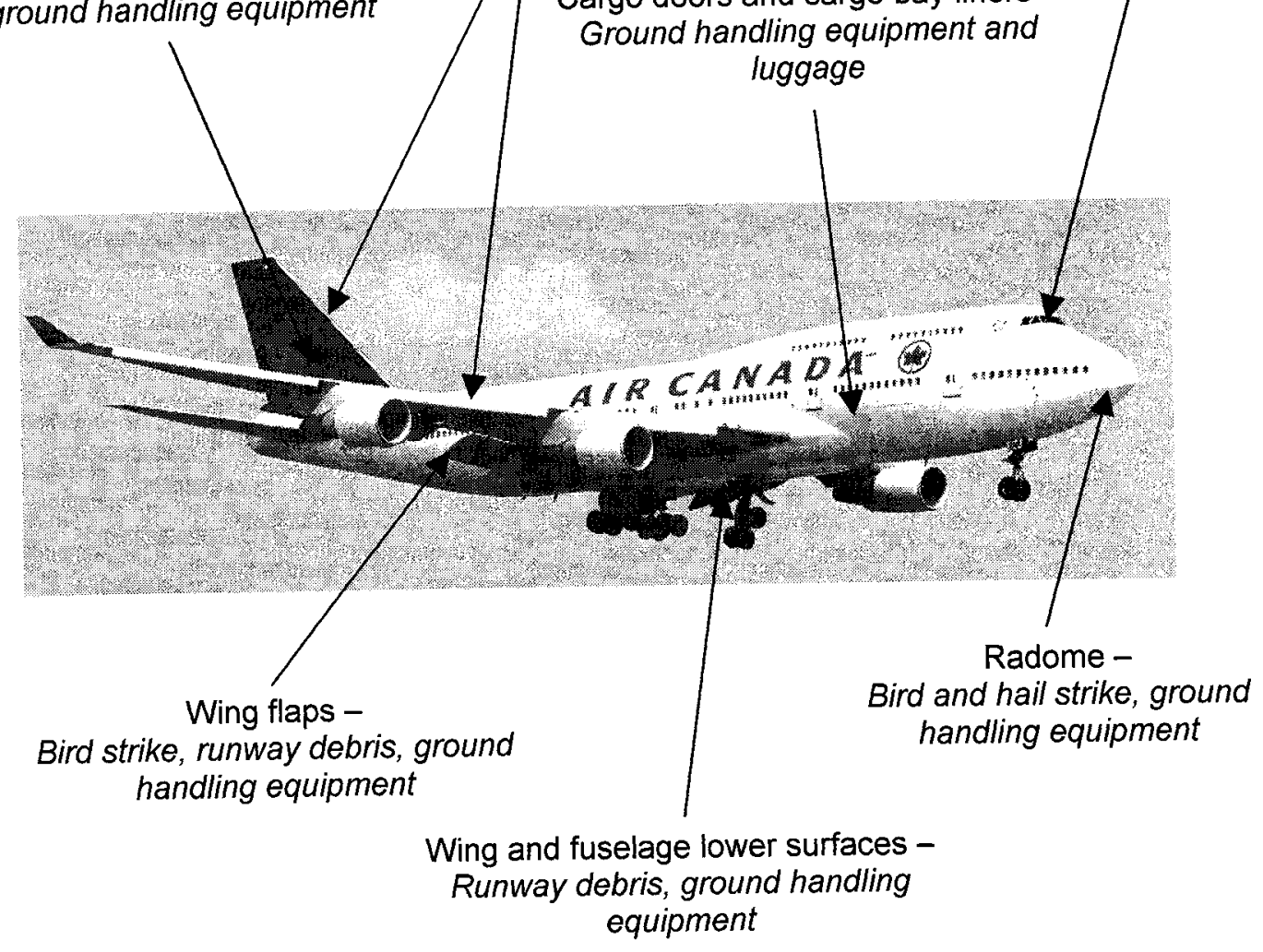

Figure 5: Areas of civil transport aircraft exposed to impact hazards.

Direction of impact

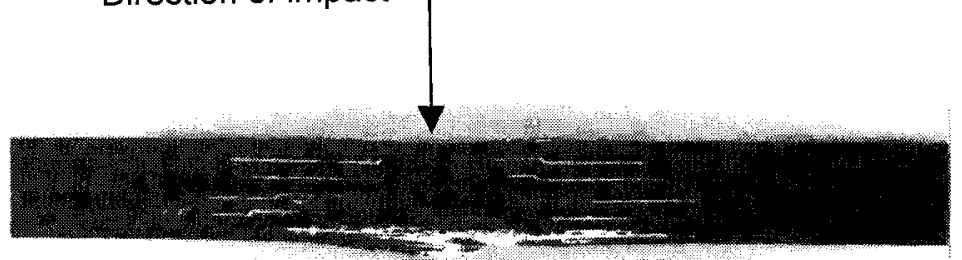

Figure 6: Formation of impact damage in a traditional composite (Majeed, 1995). 


\subsection{Thesis Objectives}

Given the above motivation, the objectives of this thesis project were:

- to conduct a detailed review of the state-of-the-art in FML technology and FML impact testing,

- to plan and carry out an extensive series of impact experiments on different varieties of GLARE, to improve the level of understanding of the impact damage modes that occur in these materials,

- to develop a practical impact damage modelling methodology for GLARE to predict the formation of damage and, if required, to use material characterization experiments to support this development, and

- to plan and carry out a series of low-velocity impact simulations on aluminum and GLARE and to compare the modelling results with the experimental tests. 


\subsection{Thesis Overview}

This thesis presents the results of an experimental and numerical investigation into the formation of impact damage in GLARE laminates. The need for a damage modelling methodology was identified during Phase I of the FML Durability Project. Work on the methodology was carried out during Phase II of the Project. The development of this methodology was not within the scope of the collaborative agreement between IAR-SMPL and Bombardier Aerospace. Persons wishing to employ the methodology should contact the author through his supervisor at Carleton University.

Where appropriate, each Chapter contains a review of the relevant literature and the state-of-the-art. This method for presenting the background information was chosen to follow the same path as the development of the experimental program and the modelling methodology. The thesis is organized into the following chapters:

Chapter 2 - review of impact tests on FMLs and results of impact testing.

Chapter 3 - review of modelling techniques for FMLs and composites; overview of the proposed damage modelling methodology; review of the LS-DYNA finite element code.

Chapter 4 - results of low-velocity impact simulations of aluminum.

Chapter 5 - delamination characterization test results.

Chapter 6-development of a prepreg material subroutine in LS-DYNA.

Chapter 7 - results of simulations of low-velocity impacts on GLARE FMLs.

Chapter 8-discussion of results of the experimental and modelling work.

Chapter 9 - conclusions from the project.

Chapter 10 - recommendations for future work and follow-on projects. 


\section{CHAPTER 2}

\section{IMPACT TESTING OF FMLS}

Any material, particularly laminates such as FMLs and traditional composites, must be subjected to stringent qualification programs before being used in airframes. One area of concern is the susceptibility of materials to impact damage from in service hazards as described in Section 1.4. To determine the extent of internal damage in GLARE from impact, a series of low-velocity impact tests was conducted. A review of historical impact test methods and results was conducted to guide the development of the test method applied in the present project. 


\subsection{Review of Impact Testing of FMLs}

A large range of "standard" test procedures for composites and FMLs is described in the literature by groups that have developed their own methods or variations on existing methods. This review will present test methods and test data from a variety of these sources. Figure 7 shows two typical impact test geometries with definitions.

Ideally, the impact test fixture used should employ a coupon size and geometry that is representative of typical aircraft structures. The coupon may represent a generic region of fuselage skin between a pair of stringers and a pair of frames, or a specific component such as a radar bulkhead. Small coupons may not accurately represent such structures. Large coupons are more expensive and require more material, so fewer can be tested. Thus, there is a trade-off between the size of the specimen and the number of specimens. A choice must also be made as to what shape the opening in the test fixture should have. Square fixture openings may cause undesirable stress concentrations in the corners. Circular specimens produce deformation patterns that are relatively free of fixture induced effects but may not be entirely representative of all aircraft structures.

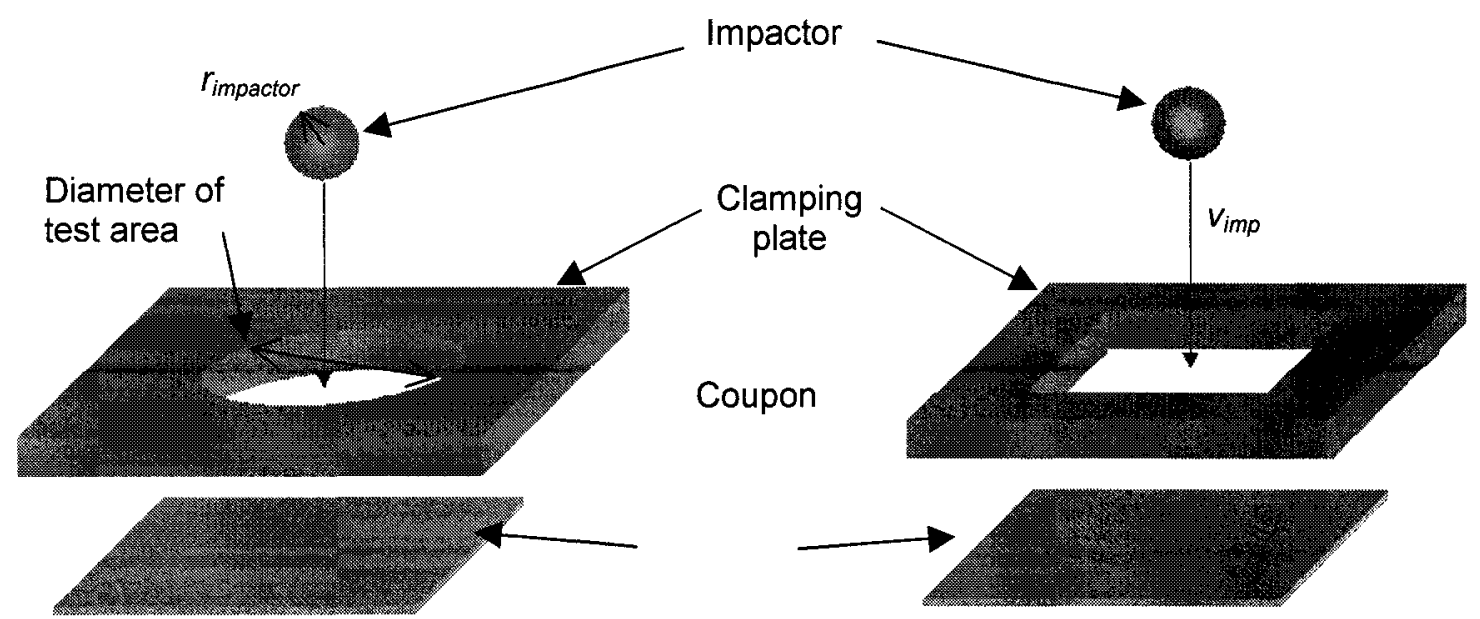

Figure 7: Basic impact test fixture geometries. 


\subsubsection{Coupon Impact Test Methods and Results}

The amount of published work on impact testing of FMLs is steadily increasing as is evident from Table 3. Much of the early work focused on ARALL (Johnson, 1986, Vlot, 1987, Hoogsteden, 1992, Wu and Sun, 1993; and Sun et al., 1993). Later, the focus shifted to GLARE (Vlot, 1987, 1993a, 1993b, 1996, Vlot et al., 1998, Poston, 1994; Liaw, 2001), aluminum-lithium and carbon fibre (ALLIC) (Freischmidt, 1994), TiGr laminates ( $\mathrm{Li}$ and Johnson, 1998) and carbon fibre-reinforced aluminum (CARE or CARALL) (Vlot, 1993b, Gregory and Roebroeks, 1991). Low-velocity impact tests are typically conducted using a drop tower or a pendulum apparatus. Pressurized gas guns are used to perform tests requiring velocities approaching ballistic levels. There are also tests that examine airframe response to specific types of impactors, such as birds.

Table 3: Summary of impact tests conducted to date on FMLs.

\begin{tabular}{|c|c|c|c|c|c|}
\hline Reference & $\begin{array}{l}\text { Materials } \\
\text { Tested }\end{array}$ & Test Type & $r_{\text {impactor }}$ & $\begin{array}{l}\text { Coupon } \\
\text { Size }\end{array}$ & $\begin{array}{c}\text { Test Area } \\
\text { Dimensions }\end{array}$ \\
\hline $\begin{array}{l}\text { Gregory and } \\
\text { Roebroeks, } 1991\end{array}$ & $\mathrm{H}$ & $\begin{array}{l}\text { Drop and gas } \\
\text { gun }\end{array}$ & (Iiim) & $\begin{array}{c}101.6 x \\
101.6\end{array}$ & $\frac{\text { (IIII) }}{\text { Not available }}$ \\
\hline Vlot, 1993a & $A, C, E, F, G$ & Drop & 7.5 & $100 \times 100$ & $\phi 80$ \\
\hline Vlot, 1993b & $A, C, D, E, G$ & Drop & 7.5 & $150 \times 150$ & $100 \times 100$ \\
\hline Vlot, 1993b & $\begin{array}{c}A, B, C, D, E \\
F, G, H, J\end{array}$ & Drop & 5 and 7.5 & $100 \times 100$ & $\phi 80$ \\
\hline Li et al., 1996 & $M$ & Charpy & $\mathrm{N} / \mathrm{A}$ & $12 \times 55$ & Not applicable \\
\hline Vlot, 1987 & $A, C, E, F, G$ & Drop & 7.5 and 0.5 & $120 \times 120$ & $\phi 80$ \\
\hline Johnson, 1986 & C, G & Drop & 12.7 & $76 \times 406$ & $\phi 50.8$ \\
\hline Hoogsteden, 1992 & $\mathrm{G}$ & Pendulum & 6.35 & $127 \times 254$ & Not available \\
\hline Wu and Sun, 1993 & $G$ & Gas gun & 6.35 & $101.6 \times 25.4$ & Not available \\
\hline Sun et al., 1993 & $G$ & Gas gun & 6.35 & $101.6 \times 25.4$ & Not available \\
\hline Vlot, 1996 & $A, C, F, G$ & Pendulum & 7.5 & $125 \times 125$ & $100 \times 100$ \\
\hline Poston, 1994 & $\begin{array}{c}A, C, D, E, F, \\
K, L, N\end{array}$ & Drop and bird & 8.0 & $100 \times 150$ & $75 \times 125$ \\
\hline Vlot et al., 1998 & $\begin{array}{c}A, B, C, D, E \\
F, G, H, J\end{array}$ & Drop & 7.5 & $100 \times 100$ & $\phi 80$ \\
\hline Liaw, 2001 & $A, F, G$ & Drop & 8 & $100 \times 100$ & $\phi 76$ \\
\hline
\end{tabular}


Note that the materials in Table 3 are represented as follows: A-2024-T3, B-7075T6, C-carbon fibre reinforced polymers (CFRP), D-glass fibre reinforced polymers (GFRP), E-aramid fibre reinforced polymers (AFRP), F-GLARE, G-ARALL, HCARALL, I-ALLIC, J-monolithic polycarbonate, K-composite plus honeycomb, L-2024T3 plus honeycomb, M-BRALL, N- 2219-T62. Table 4 summarizes the relevant impact test results from several of the researchers listed in Table 3. The following paragraphs discuss some of these results in greater detail. The first cracking energy is the impact energy required to produce a crack in the unimpacted aluminum face of the FML panel.

Table 4: Review of key impact test results.

\begin{tabular}{|l|c|c|l|}
\hline \multirow{2}{*}{ Reference } & \multirow{2}{*}{ Material } & $\begin{array}{c}\text { First Cracking } \\
\text { Energy }\end{array}$ & \multirow{2}{*}{ Notes } \\
\cline { 3 - 3 } & & $\mathbf{( J )}$ & \\
\hline \multirow{2}{*}{$\begin{array}{l}\text { Gregory and } \\
\text { Roebroeks, 1991 }\end{array}$} & GLARE-3-3/2 & 18.3 & \\
\cline { 2 - 3 } & $2024-T 3$ & 17.3 & \\
\cline { 2 - 3 } & ARALL-2-3/2 & 8.5 & \\
\hline \multirow{2}{*}{ Vlot, 1993a } & GLARE-2-2/1 & 10.3 & \\
\cline { 2 - 4 } & GLARE-2-3/2 & $>14.2$ & \\
\hline \multirow{2}{*}{ Vlot, 1993b } & GLARE-3 & 18 & Quasi-static \\
\cline { 3 - 4 } & & 37 & $10 \mathrm{~m} / \mathrm{s}$ \\
\hline Vlot, 1993b & ARALL-2-2/1 & 95 & $100 \mathrm{~m} / \mathrm{s}$ \\
\hline Hoogsteden, 1992 & ARALL-1-3/2 & $14.1-15.9$ & Appearance of partial through crack \\
\hline Vlot, 1993b & ARALL-2-2/1 & 16.4 & $\begin{array}{l}\text { Stiffened panel test compared to } \\
\text { circular coupon }\end{array}$ \\
\hline
\end{tabular}

Gregory and Roebroeks (1991) described results from impact tests of GLARE. It was reported that the minimum energy required to initiate cracking in GLARE-3-3/2- was higher than for either monolithic 2024-T3 or for ARALL-2-3/2. No description of the shape of the opening in the impact fixture was provided.

Vlot (1993a) compared the impact properties of monolithic clad and unclad 2024T3 aluminum, ARALL-2-3/2, GLARE-2-2/1, GLARE-2-3/2 and two types of carbon/PEEK laminate. In these tests a square test area was used. The impact force 
versus panel deflection curves were recorded for the tests and the energy required to initiate cracking was determined. The minimum cracking energy reported for GLARE-22/1 was higher than that reported for the thicker GLARE-2-3/2 laminates. The GLARE2-2/1 result exceeded the minimum cracking energies reported for several ARALL variants by $40-50 \%$. No cracking was observed in any of the monolithic 2024-T3 samples. It was observed that GLARE-3 had a smaller internal damage size than monolithic carbon/PEEK laminates with the same thickness and areal density, defined as mass per unit area. Also, GLARE-3 had a higher minimum cracking energy than ARALL and carbon/PEEK laminates with the same areal density. The impact dent depth for the GLARE-3 specimens was the same as that of the monolithic aluminum samples. The GLARE-3-3/2 material absorbed more impact energy than monolithic 2024-T3 for the same thickness. GLARE laminates also performed comparably to cross-ply glassreinforced composites of the same thickness. First failure occurred at the same deflection but at a much lower force in the composite ( $4 \mathrm{kN}$ versus $2.75 \mathrm{kN})$.

Vlot (1993b) used a circular impact fixture to test a variety of materials including monolithic aluminum alloys, composites, CARALL, ARALL and GLARE. It was reported that GLARE-3 demonstrated a minimum cracking energy that increased as the impact velocity was increased (Table 4). The reason for this velocity dependence is that glass fibres have strain rate-dependent mechanical properties (Vlot, 1993b). Therefore, to a greater degree than observed in ARALL, the velocity and energy level of impact both have an effect on energy absorption. Interestingly, there was no difference in dent depth observed for GLARE-3 when impacted at low and high velocities for the same energy levels. 
Vlot (1996) later published data from another investigation of the impact properties of FMLs and monolithic aluminum alloys. The tested materials included monolithic 2024-T3 and 7075-T6 aluminum, several types of ARALL, several types of GLARE, CARALL, R-glass epoxy and carbon epoxy composites. The overall specimen size and impact test set-up were the same as described previously by Vlot (1993a). The impact test support fixture used circular openings with a variety of diameters and a 7.5 $\mathrm{mm}$ radius impactor. It was found that the proximity of the impact site to the edge of the opening in the test fixtures influenced the damage modes by introducing additional deformation at the edge of the dents. This behaviour was most pronounced in small diameter specimens.

There are two standard composite impact test methods from Boeing and NASA that have also been applied to FMLs. Boeing (1988) suggested a specimen-clamping fixture for impact testing as shown in Figure 8. This test uses a $152.4 \mathrm{~mm} \times 101.6 \mathrm{~mm}$ coupon clamped by four rubber clamp tips over a $127 \mathrm{~mm} \times 76.2 \mathrm{~mm}$ rectangular hole. A $12.7 \mathrm{~mm}$ diameter hemispherical impactor is specified. This type of constraint is quite different from the continuous edge clamping used by Vlot (1993a, 1993b, 1996) and does not work well. The thin FML panels can buckle between the rubber clamps since this fixture was intended for thicker composite coupons. 


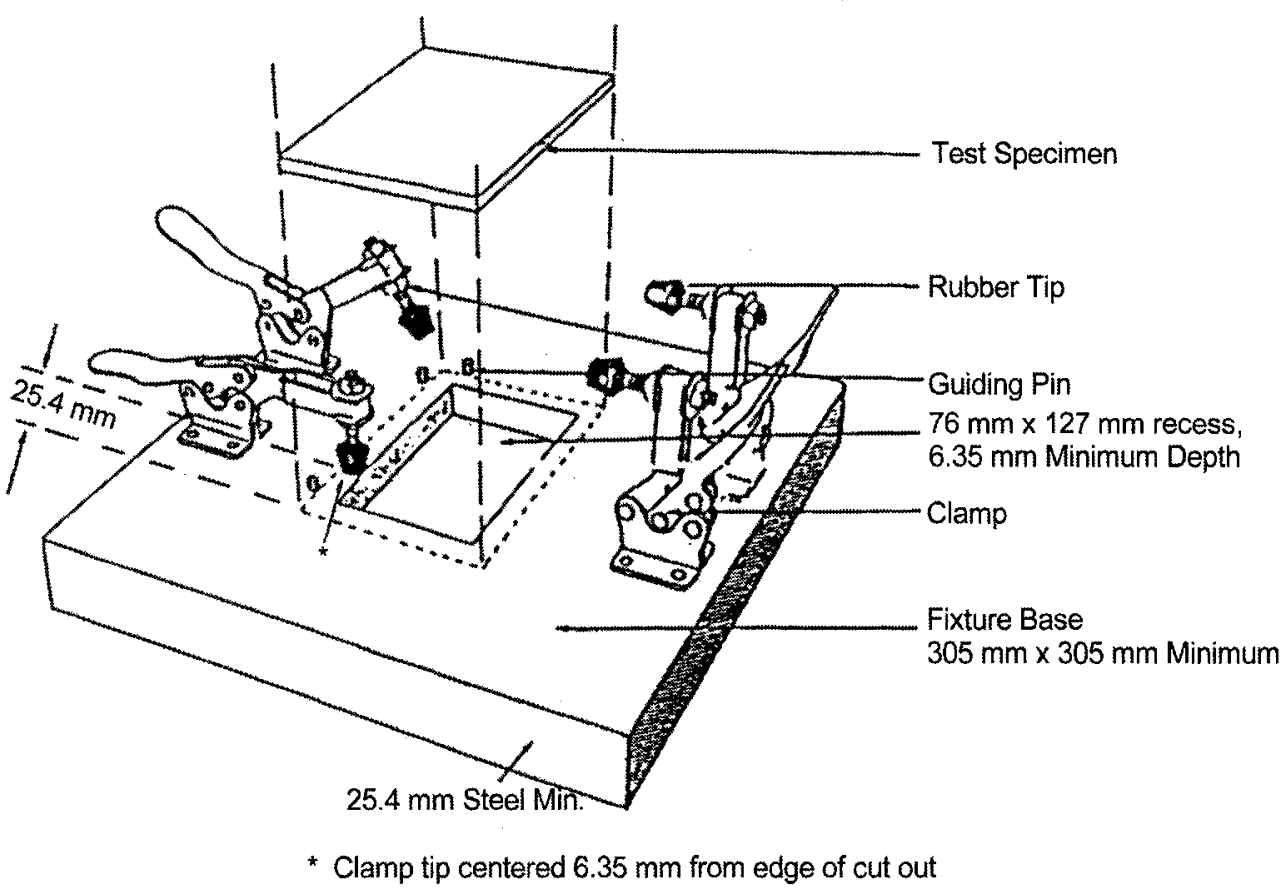

Figure 8: Boeing impact fixture (reproduced from Boeing Company, 1988).

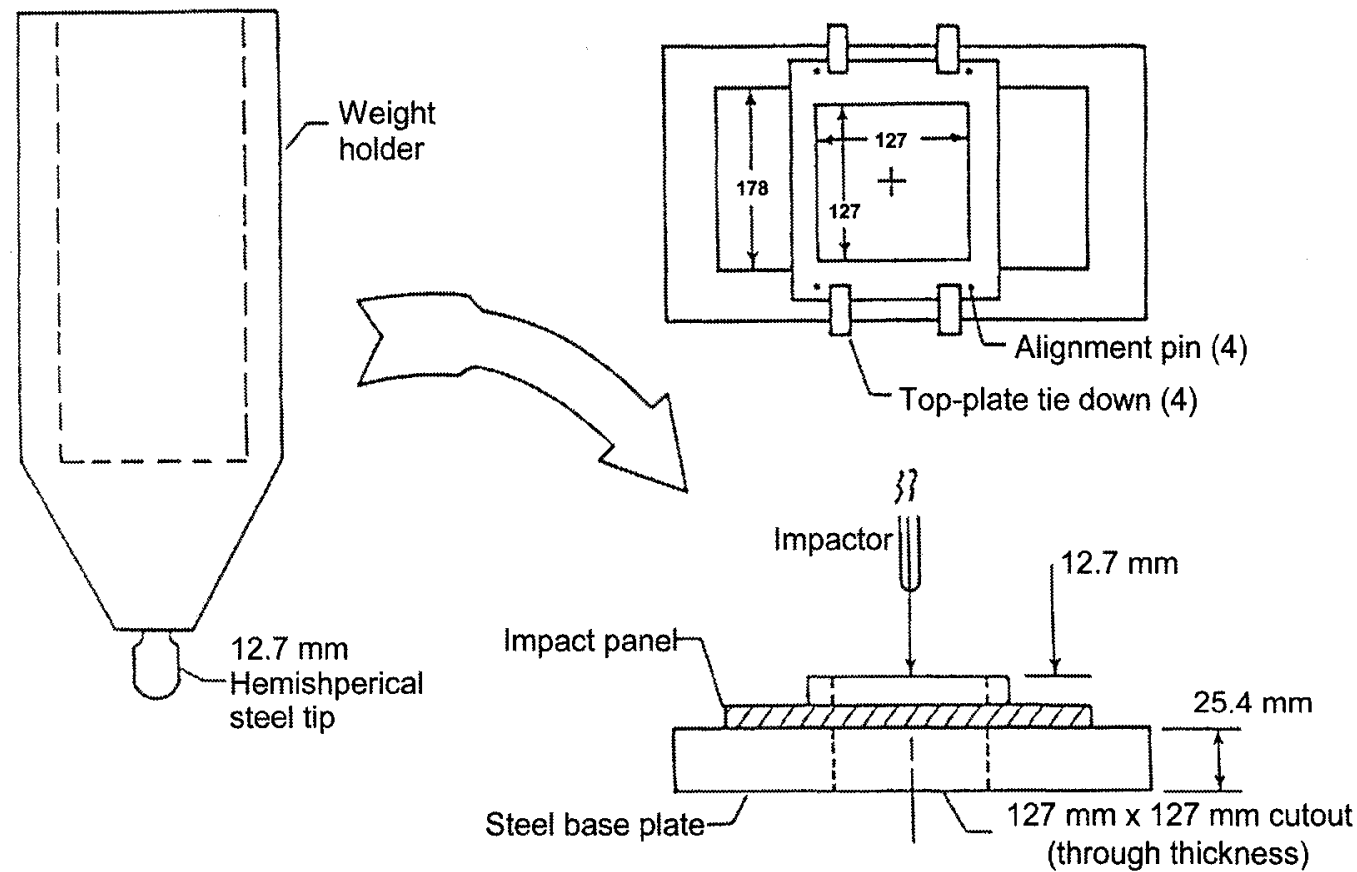

Figure 9: NASA 1092 impact fixture (reproduced from NASA, 1983). 
The other widely used test method for determining the impact resistance of composites is described by NASA (1983). This method employs larger coupons, ranging from $178 \mathrm{~mm} \times 254 \mathrm{~mm}$ to $178 \mathrm{~mm} \times 318 \mathrm{~mm}$. The specimen is clamped using a tie down plate over a $127 \mathrm{~mm} \times 127 \mathrm{~mm}$ square opening (Figure 9). For this test, a $12.7 \mathrm{~mm}$ diameter steel impactor is specified.

The variation of the NASA arrangement was used at the Air Force Research Laboratory (AFRL) to investigate the compression-after-impact strength of ARALL-1 (Hoogsteden, 1992). A total of eight specimens with two control specimens were impacted. A 3/2 lay-up was used, with three layers of aluminum 7075-T6 and two unidirectional plies of aramid fibre prepreg. The specimens were manufactured to a size of $254 \mathrm{~mm} \times 127 \mathrm{~mm}$, modified from the NASA specification of $254 \mathrm{~mm} \times 178 \mathrm{~mm}$. An instrumented pendulum impact apparatus (Figure 10) with a $12.7 \mathrm{~mm}$ diameter steel impactor was used by Hoogsteden with the NASA impact fixture.

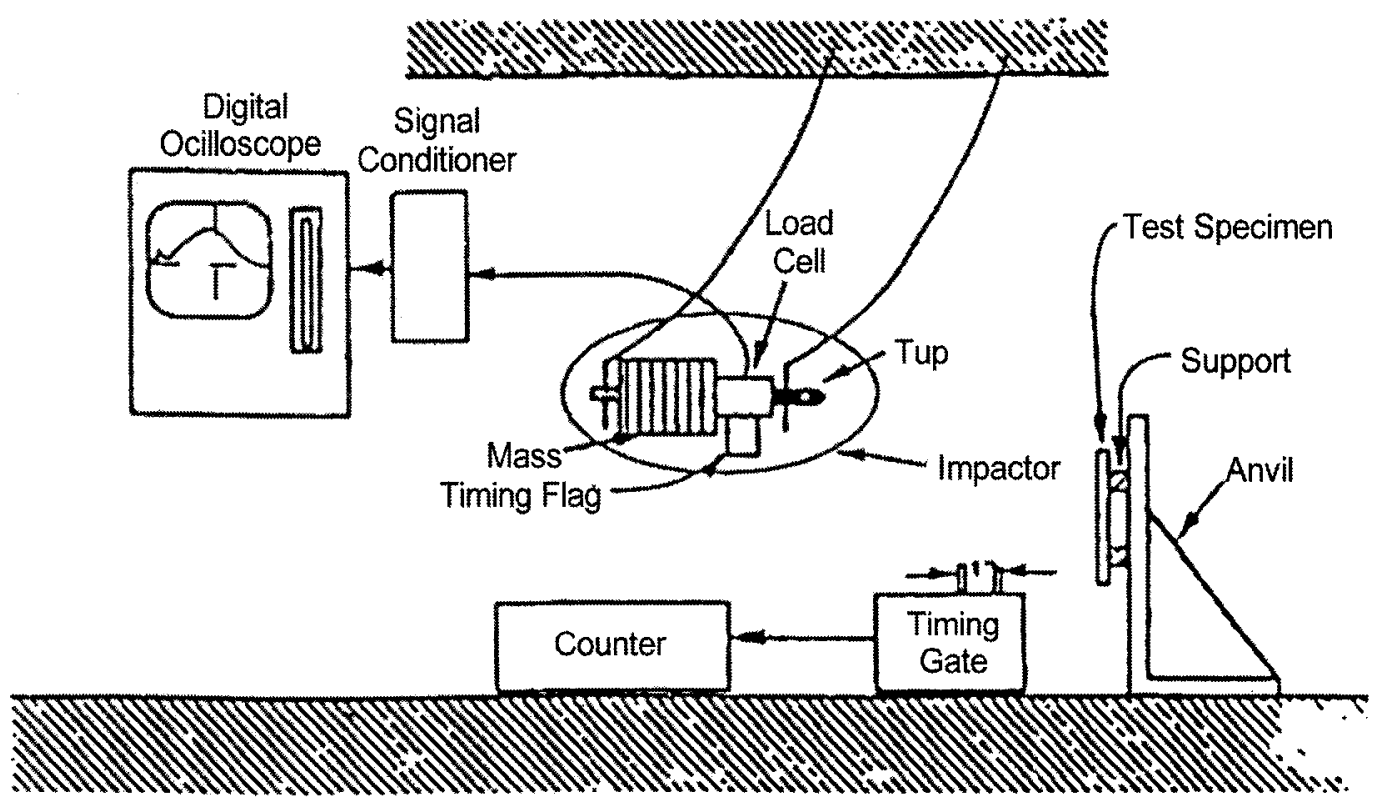

Figure 10: Wright-Patterson impact apparatus (reproduced from Hoogsteden, 1992). 
All specimens were C-scanned after impact to measure the extent of the damaged area. In comparing the ARALL-1 energy absorption data to that of graphite/epoxy and fibre glass/epoxy, Hoogsteden observed that the ARALL laminates required more impact energy to achieve the same energy absorption as the composites. The ARALL laminates absorbed a large portion of the impact energy to deform the aluminum layers. The fibres deformed elastically and thus returned a portion of the impact energy to the impactor on rebound. Also, to induce damage of a similar size, the ARALL laminates required higher impact energy than the composite materials.

\subsubsection{Component Level Impact Testing}

Vlot (1993b) described a series of impact tests conducted on stiffened panels as shown in Figure 11. A 2/1 lay-up of ARALL was used for the skin $(t=0.8 \mathrm{~mm})$ and a $3 / 2$ lay-up was used for the stringers $(\mathrm{t}=1.3 \mathrm{~mm})$. These panels more accurately represented actual aircraft structures. The ARALL stringers were manufactured using standard aluminum forming techniques, with the fibres oriented in the longitudinal direction of the stringer. These stringers were bonded to the skin using AF-163-2 adhesive. The panels were then impacted at the centre between two stiffeners. These larger panels required

more impact energy (Table 4) than circular clamped panels to induce cracking. The circular panels were tested as part of the study by Vlot (1993b) described previously. 


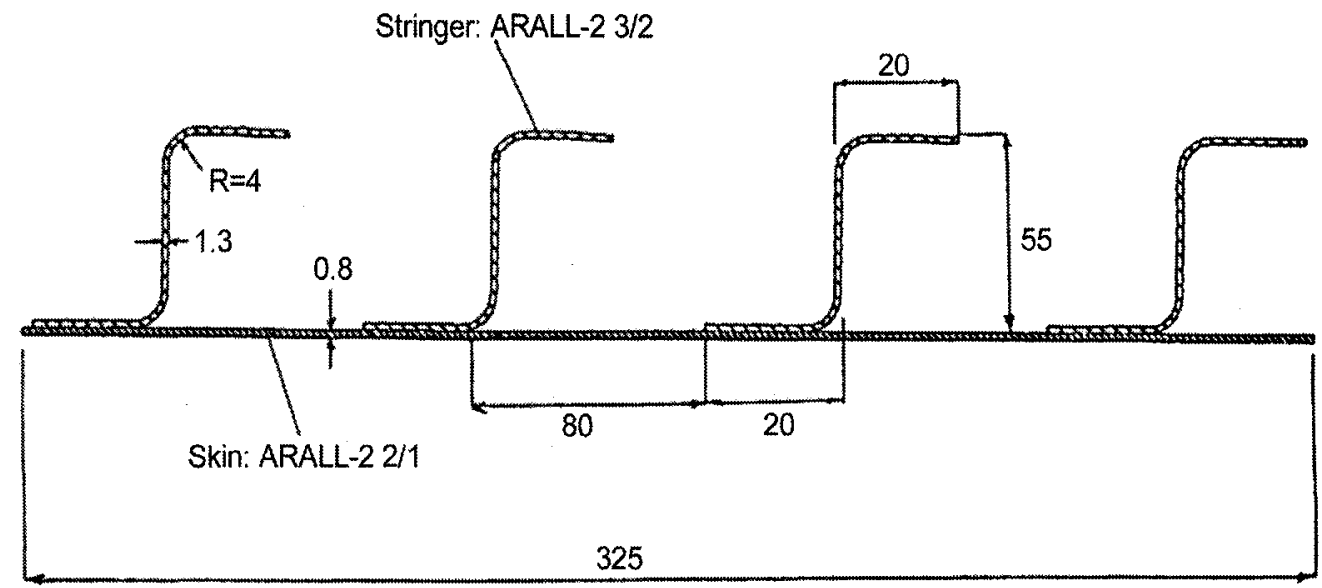

Figure 11: ARALL stiffened impact test panel (reproduced from Vlot, 1993b).

Other component level tests were carried out to select a material for the forward radome bulkhead for the Learjet 45 (Poston, 1994). In these tests several bulkhead configurations were developed using a variety of common materials. The projectile used for the impact testing was a $4 \mathrm{~kg}$ gelatinous "bird" fired from a gas gun. All test articles were $565 \mathrm{~mm}$ in diameter, with a doubler added to the periphery of some panels. Based on the deflection and energy absorption measurements, GLARE-3-5/4 was selected. 


\subsubsection{Impact Damage Measurement Techniques}

Before one can model the impact damage formed in FMLs, it is necessary to understand the various damage modes. The following techniques have been used to examine the damaged areas in FMLs (Fahr et al., 1999, Fahr et al., 2000 and Armstrong and Fawaz, 2001):

- Ultrasonic C-scan,

- Eddy current,

- Penetrant enhanced x-ray,

- Destructive inspection of the impacted specimens,

- Removal by chemical milling of the aluminum layers to expose delamination and matrix cracking in the fibre-reinforcing layers, and

- Infrared thermography.

Each of the above methods has specific advantages and disadvantages. The first two methods are non-destructive techniques. However, there is a general agreement among researchers that C-scan (Vlot, 1993b; Sun et al., 1993; and Fahr et al., 1999) is ineffective for impacted coupons due to the large plastically deformed dent that scatters the sound waves. Eddy current techniques are not affected by the shape of the specimen but are only useful for detecting cracking in the aluminum layers.

The next three techniques are all to some degree destructive. Penetrant enhanced x-ray is the least destructive of the three. This process involves drilling a small hole through the top layers of the specimen and then injecting an x-ray opaque penetrant. The x-radiographs then clearly show the extent of the delamination. However, with this two- 
dimensional imaging technique it is not possible to determine which layer has the most damage. Destructive inspection uses standard metallographic techniques to mount, cut and then polish impact specimens. Scanning electron microscopy or conventional optical microscopy can then be used to inspect the damage. This technique gives a good picture of the through-thickness damage pattern.

Another damage characterization technique developed for FMLs consists of removing the aluminum layers with a nitric or hydrochloric acid solution. The aluminum layers in GLARE are removed without damaging the epoxy/glass prepreg layers (Vlot, 1993a). The prepreg can then be examined to determine the levels of matrix cracking and fibre damage.

Infrared thermography has been used with limited success for detecting delamination in impacted FML panels (Armstrong and Fawaz, 2001) and also in FML panels subjected to post-impact fatigue (Laliberté, et al., 2001). This method proved to be qualitative and not suitable for providing detailed measurements of the damaged area.

Delfosse and Poursartip (1997) described another method used to measure the damage in impacted composite panels. A high temperature furnace was used to burn off the epoxy layers. The measured length of the broken fibres was used to determine the approximate amount of energy absorbed through fibre fracture in impacted composite panels. It was noted that this method was labour intensive and was appropriate for a limited number of specimens, rather than a large sample of impacted specimens. Also, it would not be possible to place FML panels in the furnace due to the presence of the alloy layers. The metal layers would have to be removed using the acid etch method first. This would increase the labour requirement further. 


\subsection{Low-Velocity Impact Testing of FMLs}

Based on the review of impact data and test methods, it was determined that upto-date impact resistance information using a realistic specimen size was required for GLARE. This section presents the results of low-velocity impact tests of several variants of GLARE and monolithic 2024-T3. The impact tests were conducted with the Dynatup instrumented drop weight impact tower located at IAR-SMPL.

\subsubsection{Preliminary Impact Testing}

Prior to the formal impact tests a series of preliminary tests were completed using only GLARE-3-2/1. These tests established the procedures and test matrix to be used for the formal impact test program. The NASA impact fixture described in Section 2.1.1 was initially used to support the GLARE specimens. As can be seen in Figure 12a, the square opening causes stress concentrations at the corners of the specimen that can influence the formation of damage. Therefore, a new impact fixture with a circular opening was developed that resulted in the impact damage geometry shown in Figure $12 \mathrm{~b}$. The NRC impact fixture that was developed is shown in Figure 13. This fixture reduced the effects of the boundary conditions on the impact damage patterns and provided a clamped and symmetrical boundary condition.

Details of the results of the comparative tests using the NASA and NRC fixtures were published by Laliberté et al. (1999b). Appendix A is a summary of preliminary impact results from the NRC impact fixture with various impactors. Appendix B contains the impact results from the NASA and NRC fixtures using $25.4 \mathrm{~mm}$ and 12.7 
$\mathrm{mm}$ diameter impactors. Appendix $\mathrm{C}$ contains graphical comparisons of the impact test results from the NASA and NRC test fixtures.
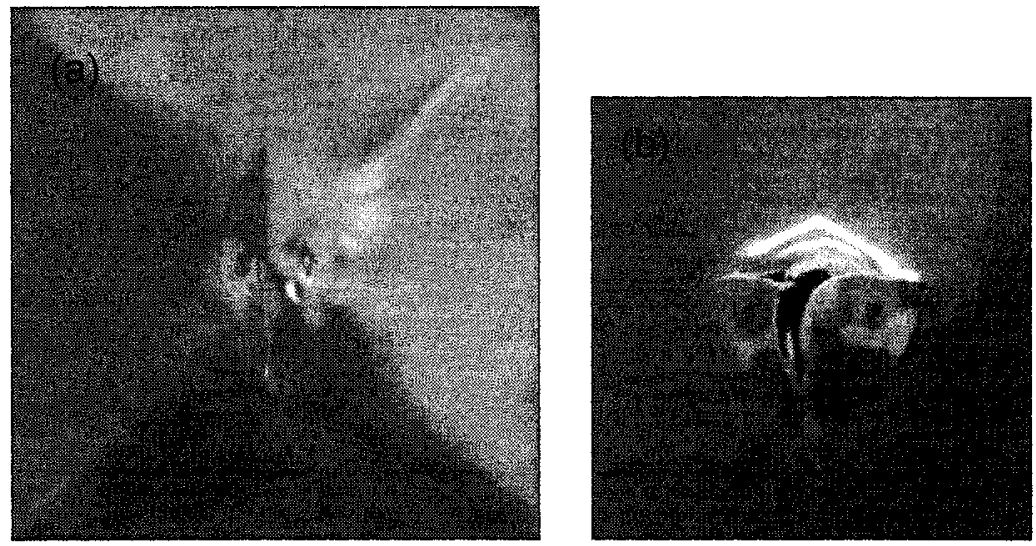

Figure 12: Comparison of damage geometry from the (a) NASA and (b) NRC impact fixtures.

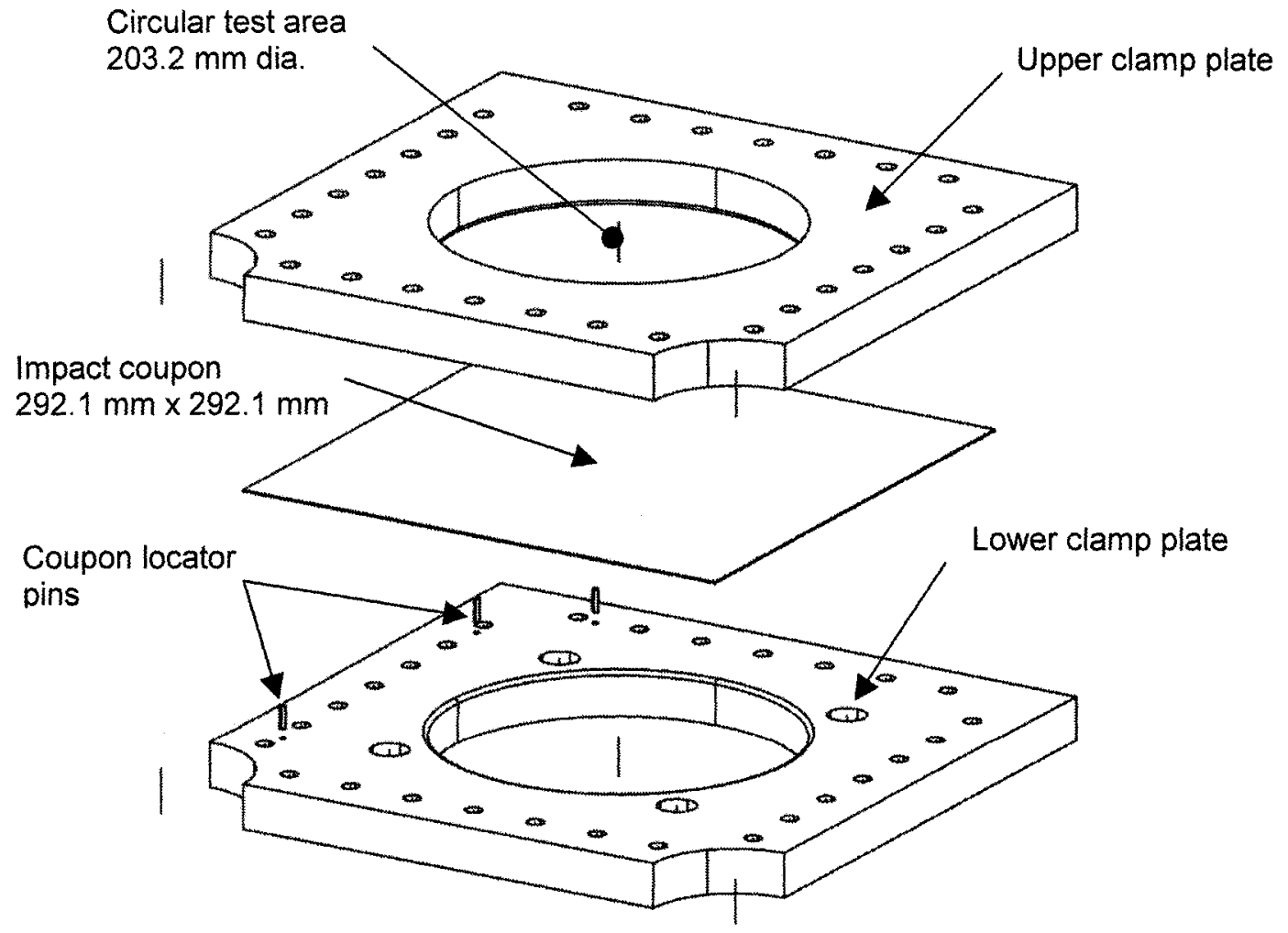

Figure 13: NRC impact fixture. 


\subsubsection{Formal Impact Testing}

Following the completion of the preliminary tests, a formal series of impact tests was carried out using three varieties of GLARE and one type of aluminum. The NRC impact fixture was used for these tests. The following materials were tested:

- aluminum 2024-T3 $t=1.02 \mathrm{~mm}, \rho_{a}=2.82 \mathrm{~kg} / \mathrm{m}^{2}$,

- GLARE-3-2/1-(0.3) $t=0.85 \mathrm{~mm}, \rho_{a}=2.17 \mathrm{~kg} / \mathrm{m}^{2}$,

- GLARE-4-2/1-(0.3) $t=0.98 \mathrm{~mm}, \rho_{a}=2.42 \mathrm{~kg} / \mathrm{m}^{2}$, and

- GLARE-5-2/1-(0.3) $t=1.11 \mathrm{~mm}, \rho_{a}=2.69 \mathrm{~kg} / \mathrm{m}^{2}$.

The GLARE-3-2/1 material was clad with a thin layer of pure aluminum on the impacted face. The remaining GLARE types were chromated for corrosion resistance. The prepreg layers consisted of Hexcel F185 epoxy with cross-plies of S2-glass unidirectional fibres providing reinforcement. Figure 14 shows the GLARE variants that were tested.

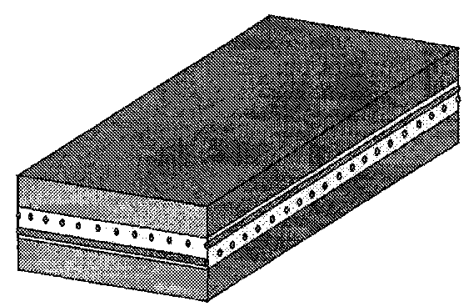

(A) GLARE-3-2/1

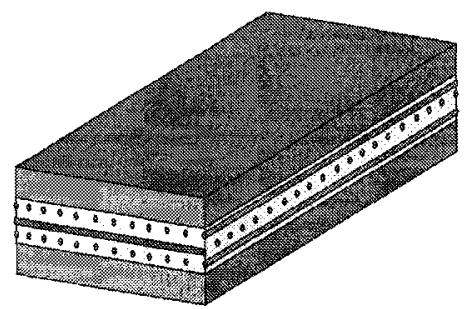

(B) GLARE-4-2/1

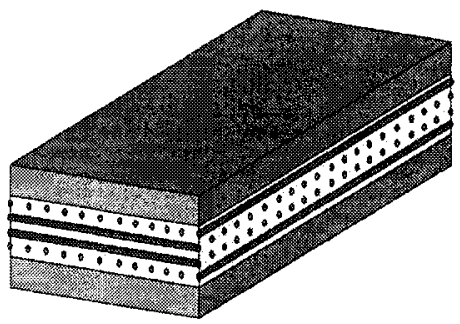

(C) GLARE-5-2/1

Figure 14: GLARE-2/1 variants subjected to impact testing. 


\subsubsection{Experimental Procedure}

\section{Test Matrix}

The test matrix employed for the impact testing is shown in Table 5. The undamaged control specimens provided baseline data for post-impact strength tests. Energy levels were selected based on the preliminary impact test results. The energies represent impact events that would produce the following progressive damage states:

- plastically deformed dent with no delamination (typically for $E_{i m p} \leq 25 \mathrm{~J}$ ),

- plastically deformed dent with delamination (typically for $25 \mathrm{~J}<E_{\text {imp }}<55$ J), and

- puncture (typically for $E_{i m p}>55 \mathrm{~J}$ ).

During the preliminary testing, specimens were x-rayed to determine levels of internal damage to help establish these energy levels.

Table 5: Test matrix for formal FML impact tests.

\begin{tabular}{|c|c|c|c|c|c|c|c|c|c|}
\hline \multirow{2}{*}{ Material } & \multirow{2}{*}{ Control } & \multicolumn{7}{|c|}{ Impact Energy Level (J) } & \multirow{2}{*}{ Total } \\
\cline { 3 - 11 } & & $\mathbf{1 5}$ & $\mathbf{2 5}$ & $\mathbf{3 5}$ & $\mathbf{4 5}$ & $\mathbf{5 5}$ & $\mathbf{6 0}$ & $\mathbf{6 5}$ & \\
\hline \hline 2024-T3 & 2 & 0 & 6 & 6 & 6 & 6 & 6 & 0 & $\mathbf{3 2}$ \\
\hline GLARE-3 & 2 & 6 & 6 & 6 & 6 & 6 & 0 & 0 & 32 \\
\hline GLARE-4 & 2 & 0 & 6 & 6 & 6 & 6 & 6 & 0 & 32 \\
\hline GLARE-5 & 2 & 0 & 6 & 6 & 6 & 6 & 0 & 6 & 32 \\
\hline Total & 6 & 6 & 24 & 24 & 24 & 24 & 12 & 6 & \\
\hline
\end{tabular}

\section{Drop Tower Data Collection Methods}

Poon and Benak (1986) prepared a complete description of the drop tower (Figure 15), its capabilities and its limitations. A block diagram of the data acquisition system is shown in Figure 16. 


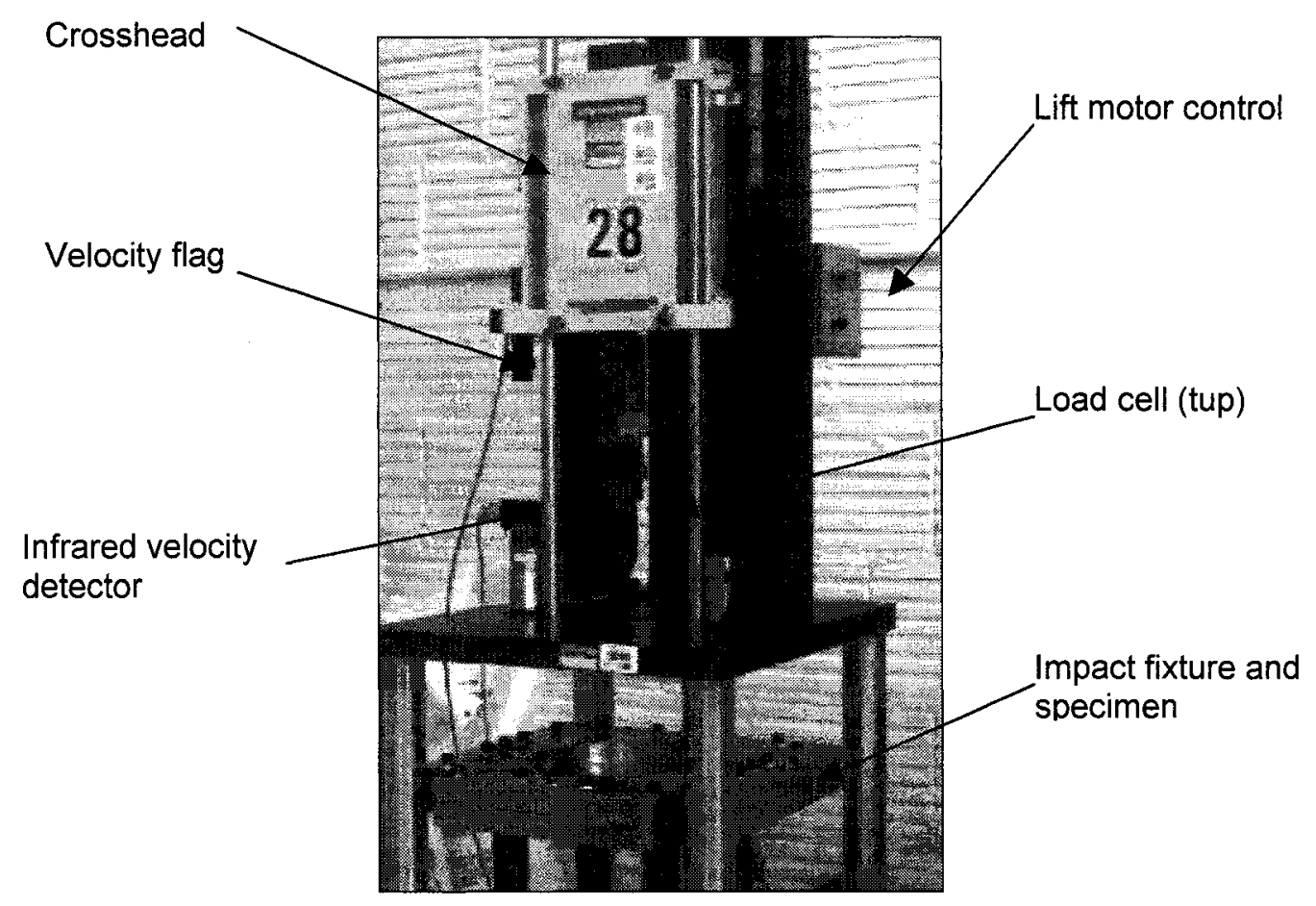

Figure 15: NRC Dynatup drop-weight impact tower with the NRC fixture.

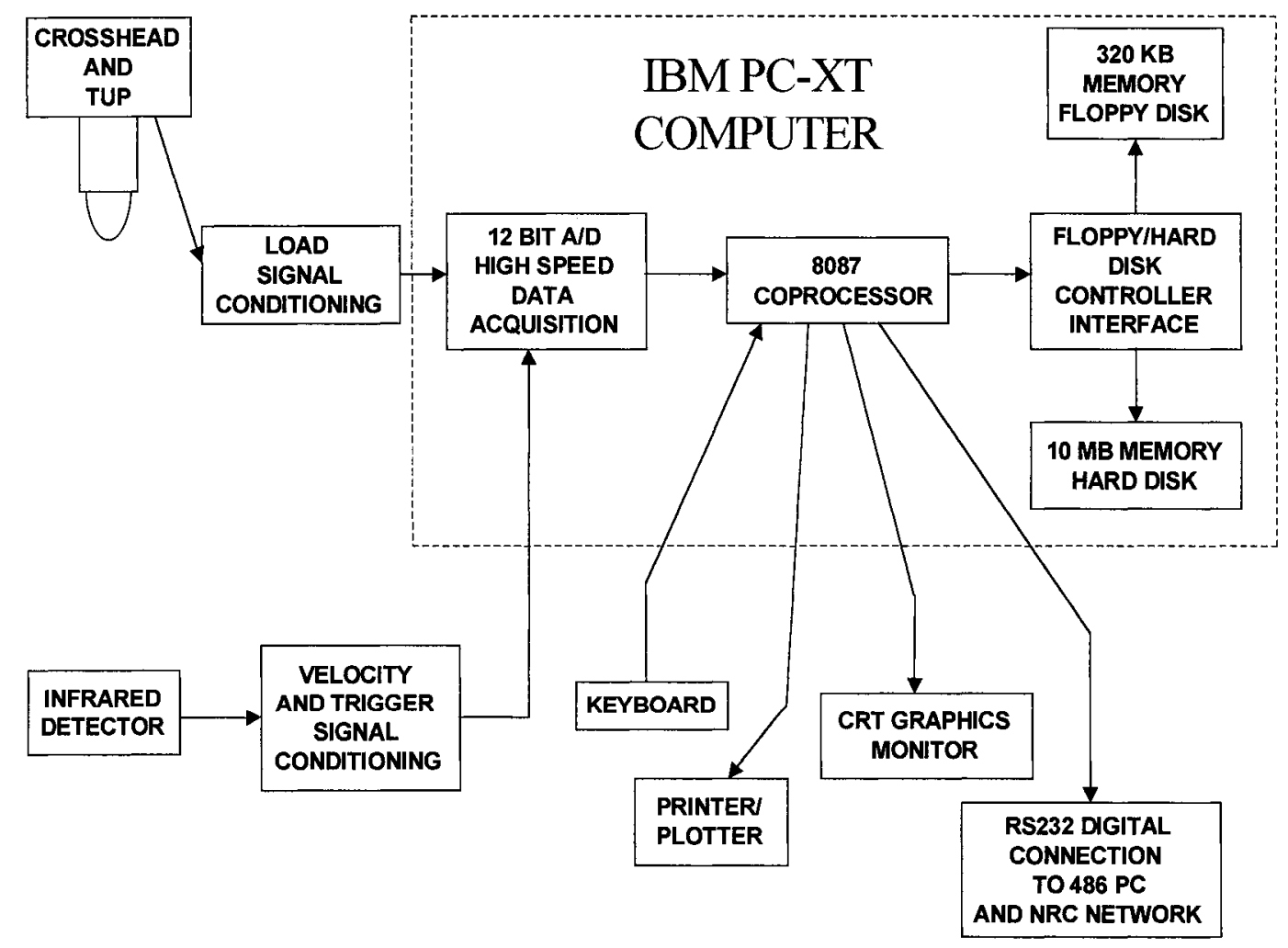

Figure 16: Block diagram for Dynatup Model 730-I impact system. 
The Dynatup drop tower is interfaced with an IBM XT personal computer through a commercial data acquisition card. The data acquisition software is triggered when a metal flag passes through a timing gate. The sampling rate is determined by the user and is based on the duration of the impact event. Event durations can range from $10 \mathrm{~ms}$ to $100 \mathrm{~ms}$ with 1024 data points recorded regardless of the event duration. The velocity at impact is measured by the infrared timing gate triggered by the passage of a metal flag mounted on the crosshead. The location of the timing gate is adjusted so that it records the velocity of the crosshead at the moment of impact. The instrumented load cell records impact force and then uses this information to calculate deflection, instantaneous velocity and absorbed energy with the following equations (Poon and Benak, 1986): the displacement of the tup, $d_{i}$ :

$$
d_{i}=d_{i-1}+\overline{V_{i}} \Delta t
$$

average velocity:

$$
(\bar{V})=\frac{1}{2}\left(V_{i}+V_{i-1}\right)
$$

instantaneous velocity at any time interval $i$ :

$$
V_{i}=V_{i-1}+\left(g-\frac{P_{i-1}+P_{i}}{2 m}\right) \Delta t
$$

where $m$ is the impactor mass and $P$ is the force. The absorbed energy is thus:

$$
\left(E_{o}\right)_{i}=\left(E_{o}\right)_{i-1}+\frac{P_{i-1}+P_{i}}{2}\left(V_{i}\right) \Delta t
$$

All panels were clamped between the top and bottom plates of the fixture with $3 / 8$ in. machine bolts. The bolts were tightened and loosened in a uniform pattern with a torque of $10.2 \mathrm{~N}-\mathrm{m}$ to provide a consistent clamping force for all of the samples. 
Several panels from each sample group were instrumented with strain gauges to collect instantaneous strain data from each test. Table 6 summarizes the energy levels at which the instrumented panels were impacted. A Nicolet model Pro-20 four-channel digital oscilloscope was employed to capture the strain data. Pacific model 1073 strain gauge amplifiers were used to amplify and condition the signals from the strain gauges.

Table 6: Summary of instrumented and impacted GLARE specimens.

\begin{tabular}{|l|c|c|c|c|c|c|c|c|}
\hline \multirow{2}{*}{ Material } & \multicolumn{7}{|c|}{ Impact energy level (J) } & \multirow{2}{*}{ Total } \\
\cline { 2 - 10 } & $\mathbf{1 5}$ & $\mathbf{2 5}$ & $\mathbf{3 5}$ & $\mathbf{4 5}$ & $\mathbf{5 5}$ & $\mathbf{6 0}$ & $\mathbf{6 5}$ & \\
\hline \hline 2024-T3 & 0 & 0 & 0 & 0 & 0 & 0 & 0 & 0 \\
\hline GLARE-3 & 1 & 1 & 1 & 1 & 1 & 0 & 0 & 5 \\
\hline GLARE-4 & 0 & 1 & 1 & 1 & 1 & 1 & 0 & 5 \\
\hline GLARE-5 & 0 & 1 & 1 & 1 & 1 & 0 & 1 & 5 \\
\hline Total & 1 & 3 & 3 & 3 & 3 & 1 & 1 & 15 \\
\hline
\end{tabular}

\section{Impact Damage Inspection}

Table 7 contains the post-impact damage characterization matrix. Specimens were $\mathrm{x}$-rayed using the penetrant enhanced technique described in Section 2.1.3. Additional specimens were selected from each energy level for destructive inspection. The results of the post-impact examinations are presented in Section 2.2.4.

Table 7: Summary of inspected specimens.

\begin{tabular}{|l|c|c|c|c|c|c|c|c|}
\hline \multirow{2}{*}{ Inspection Method } & \multicolumn{7}{|c|}{ Impact Energy Level (J) } & Total \\
\cline { 2 - 10 } & $\mathbf{1 5}$ & $\mathbf{2 5}$ & $\mathbf{3 5}$ & $\mathbf{4 5}$ & $\mathbf{5 5}$ & $\mathbf{6 0}$ & $\mathbf{6 5}$ & \\
\hline \hline Total X-Rayed & 2 & 6 & 6 & 6 & 6 & 2 & 2 & \\
\hline GLARE-3 & 2 & 2 & 2 & 2 & 2 & 0 & 0 & 10 \\
\hline GLARE-4 & 0 & 2 & 2 & 2 & 2 & 2 & 0 & 10 \\
\hline GLARE-5 & 0 & 2 & 2 & 2 & 2 & 0 & 2 & 10 \\
\hline Total Destructively & 1 & 3 & 3 & 3 & 3 & 1 & 1 & \\
\hline Inspected & 1 & 1 & 1 & 1 & 1 & 0 & 0 & 5 \\
\hline GLARE-3 & 0 & 1 & 1 & 1 & 1 & 1 & 0 & 5 \\
\hline GLARE-4 & 0 & 1 & 1 & 1 & 1 & 0 & 1 & 5 \\
\hline GLARE-5 & 3 & 9 & 9 & 9 & 9 & 3 & 3 & 5 \\
\hline Overall Totals & & & & & \\
\hline
\end{tabular}




\subsubsection{Impact Test Results}

The impact test results are presented in several forms. First, in terms of the amount of energy absorbed by the panel as a function of impact energy. Next, the impact data was normalized in terms of the areal density of the panels to rank the laminates on their relative performance. Another method for displaying the impact data is maximum impact force versus impact energy. Several force versus time and strain versus time traces for representative impact tests are presented. Complete tabulated results of the impact tests can be found in Appendix D.

\section{Absorbed Energy}

Figure 17 shows the progression of absorbed energy with increasing impact energy. Prior to puncture the amount of absorbed energy is below $100 \%$, indicated by the straight line on the graph. Energy is absorbed first by plastic deformation of the panel and by matrix cracking. Then, delamination damage occurs and absorbs a portion of the impact energy. Finally, the panels are punctured by the impactor and energy is absorbed by fibre and aluminum fracture. The unabsorbed energy is transferred back to the impactor or converted to sound and heat. Typically, the energy converted to heat and sound is quite small (Vlot, 1993b). It is not obvious from the experiments what fraction of energy is absorbed by these mechanisms. Impact simulations are required to determine this.

The GLARE-3 and GLARE-5 laminates puncture in a similar manner; cracks develop in the $0^{\circ}$ and $90^{\circ}$ fibre directions. However, in punctured GLARE-4 a single unidirectional crack is formed in the $0^{\circ}$ direction rather than a complete puncture. As the impact energy increases so does the length of the crack. The GLARE-4 panels have 30 
percent of the fibres perpendicular to the rolling direction and 70 percent parallel to the rolling direction creating difference in stiffness that leads to the observed crack geometry. Once the GLARE-4 panel is punctured a portion of the energy is still returned to the impactor. In the GLARE-3 and GLARE-5 panels there is almost no rebound of the impactor following puncture. At impact energies exceeding the puncture threshold, the percentage of absorbed energy decreases. This occurs because the impactor passes through the specimen and retains some of its kinetic energy. The aluminum 2024-T3 panels were not punctured during this test program, so direct comparison of puncture initiation energies is not possible.

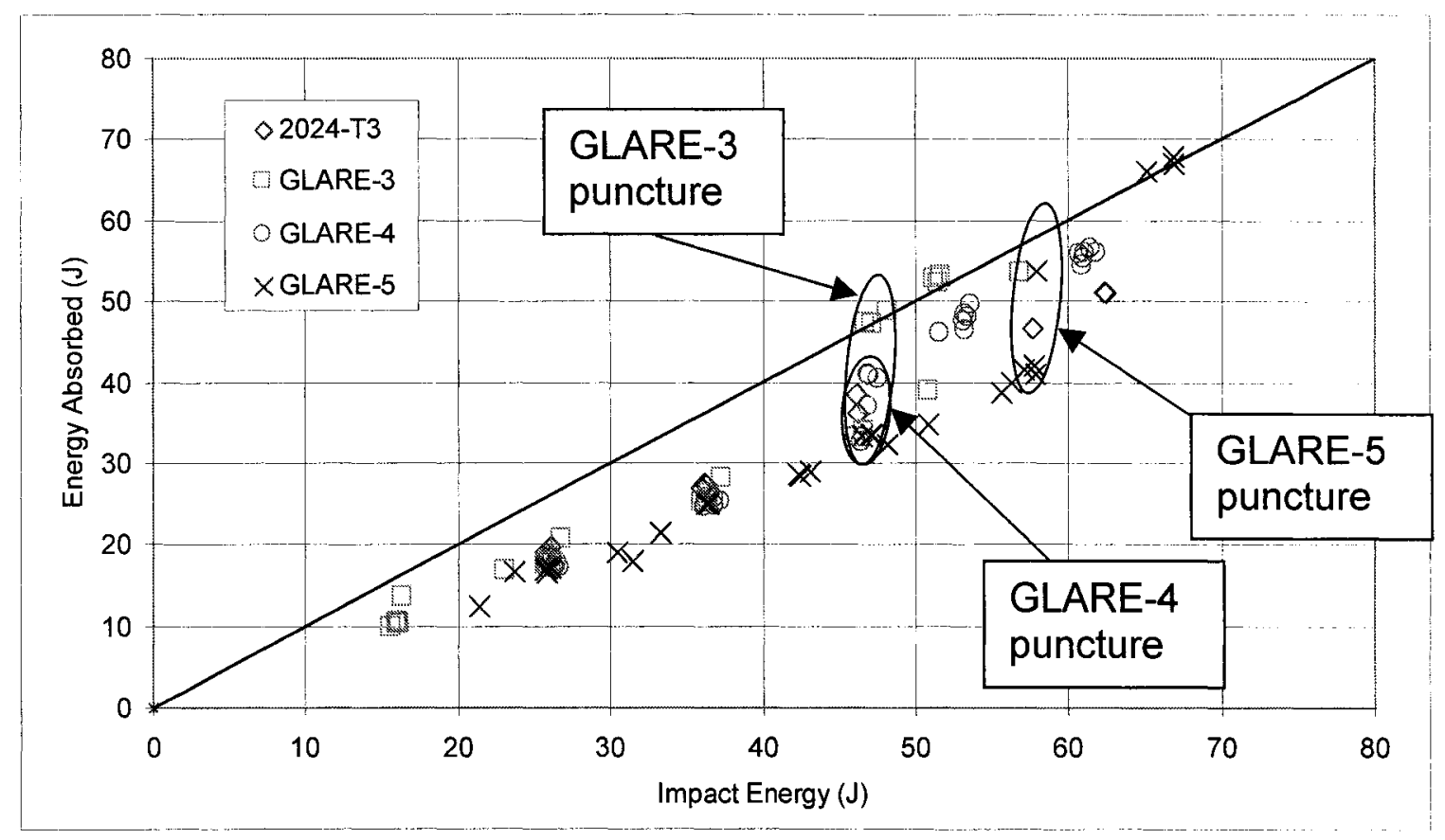

Figure 17: Absorbed energy versus impact energy for GLARE and 2024-T3.

Figure 18 shows the absorbed energy normalized by areal density as a function of the impact energy for various laminates. It can be seen that GLARE-5 absorbed the least amount of energy for its areal density, and therefore suffered the least amount of damage. This result was expected since GLARE-5 was reportedly optimized for impact loading. 


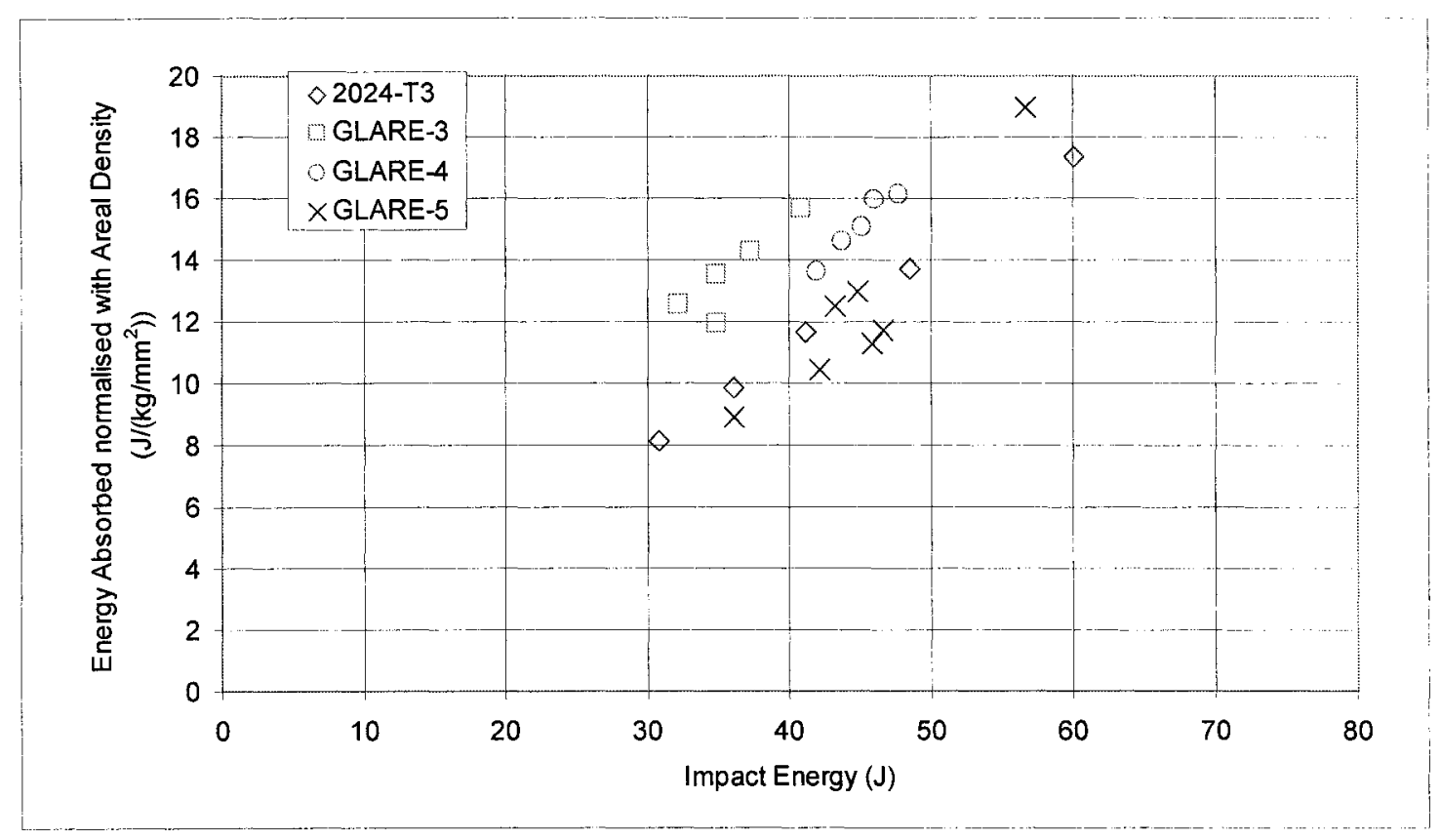

Figure 18: Normalized absorbed energy versus impact energy. Each data point in the above graph is an average of 5 tests.

\section{Peak Impact Force}

The peak impact force is shown in Figure 19 as a function of the impact velocity. The impact force varies almost linearly with the velocity of the crosshead. However, at higher velocity levels the impact force begins to level off in the GLARE-3 and GLARE-4 punctured panels. The capacity of the panels to resist the impactor was exceeded once puncture occurred. The GLARE-5 panels did not have the same levelling off of peak impact force at higher velocities. 


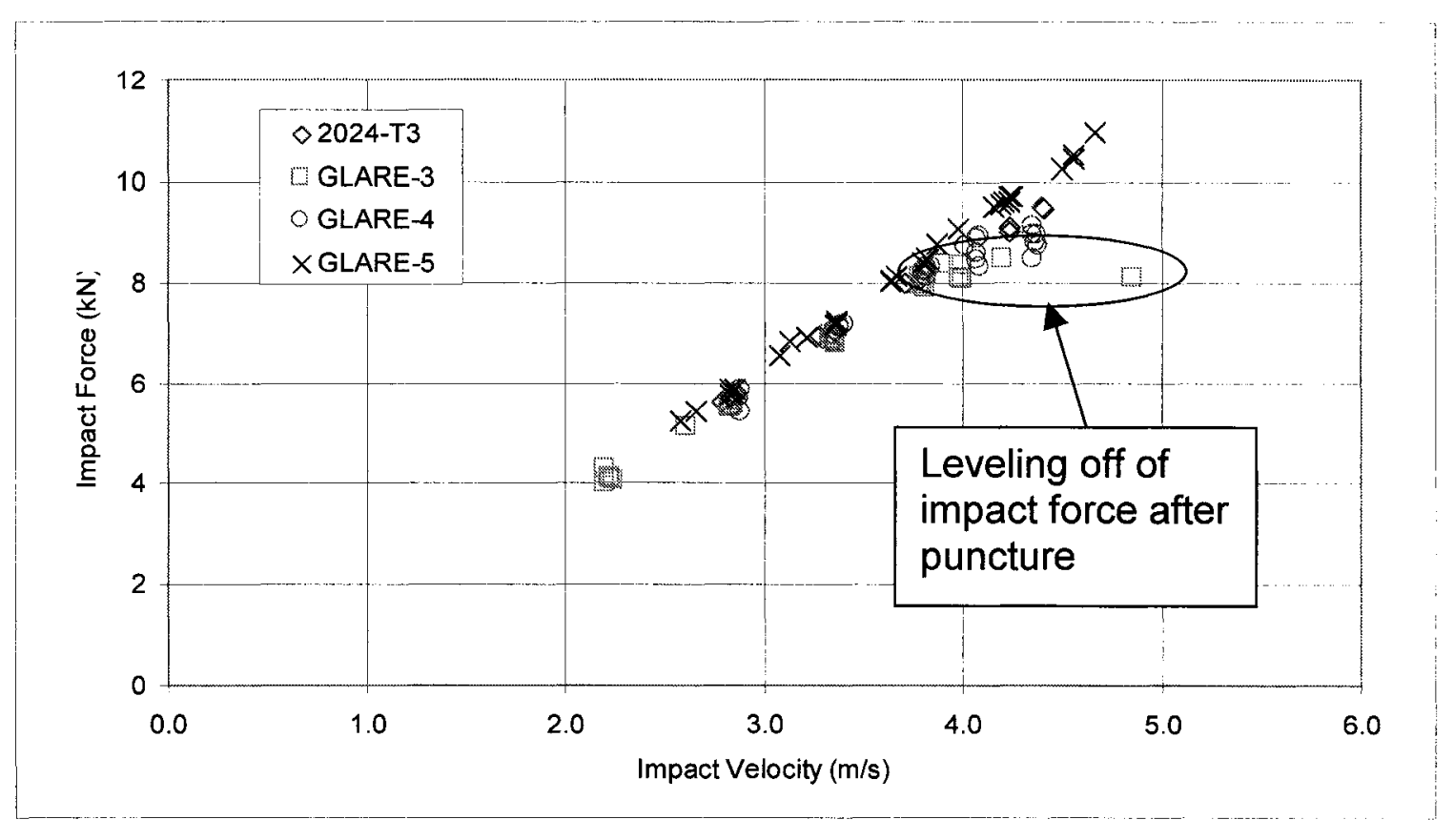

Figure 19: Impact force versus impact velocity.

Figures 20 to 23 are sample load traces for each of the materials tested. The initial cyclic variations observed in all the load traces are the result of oscillations in the panel induced by impact. The load traces for aluminum shown in Figure 20 are smooth and well defined. The shape of the impact force response curves remains consistent as the impact energy increases. However, the overall event time decreases. For GLARE-3 and GLARE-5 the load drops off sharply (Figures 21 and 22) once the panel is fully penetrated. 


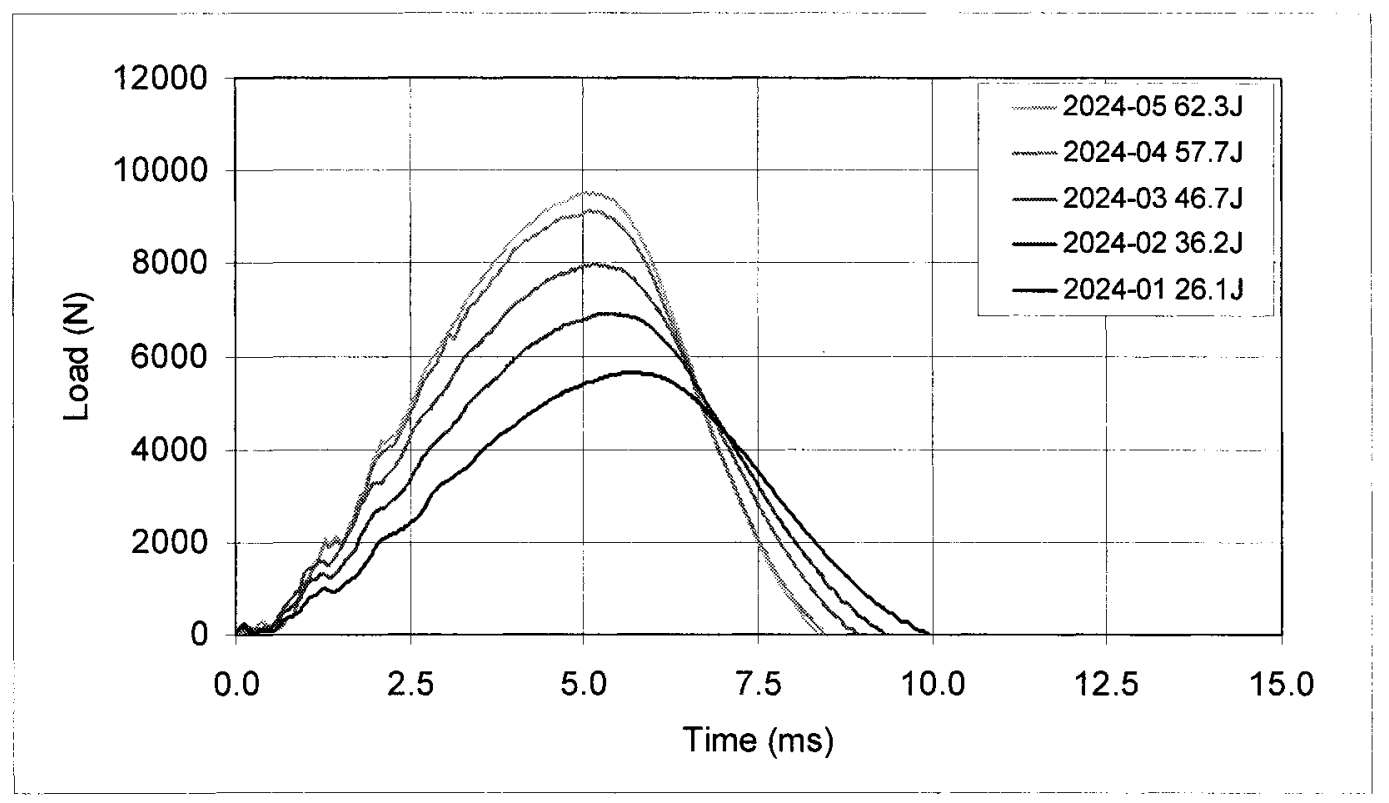

Figure 20: Aluminum 2024-T3 sample load traces.

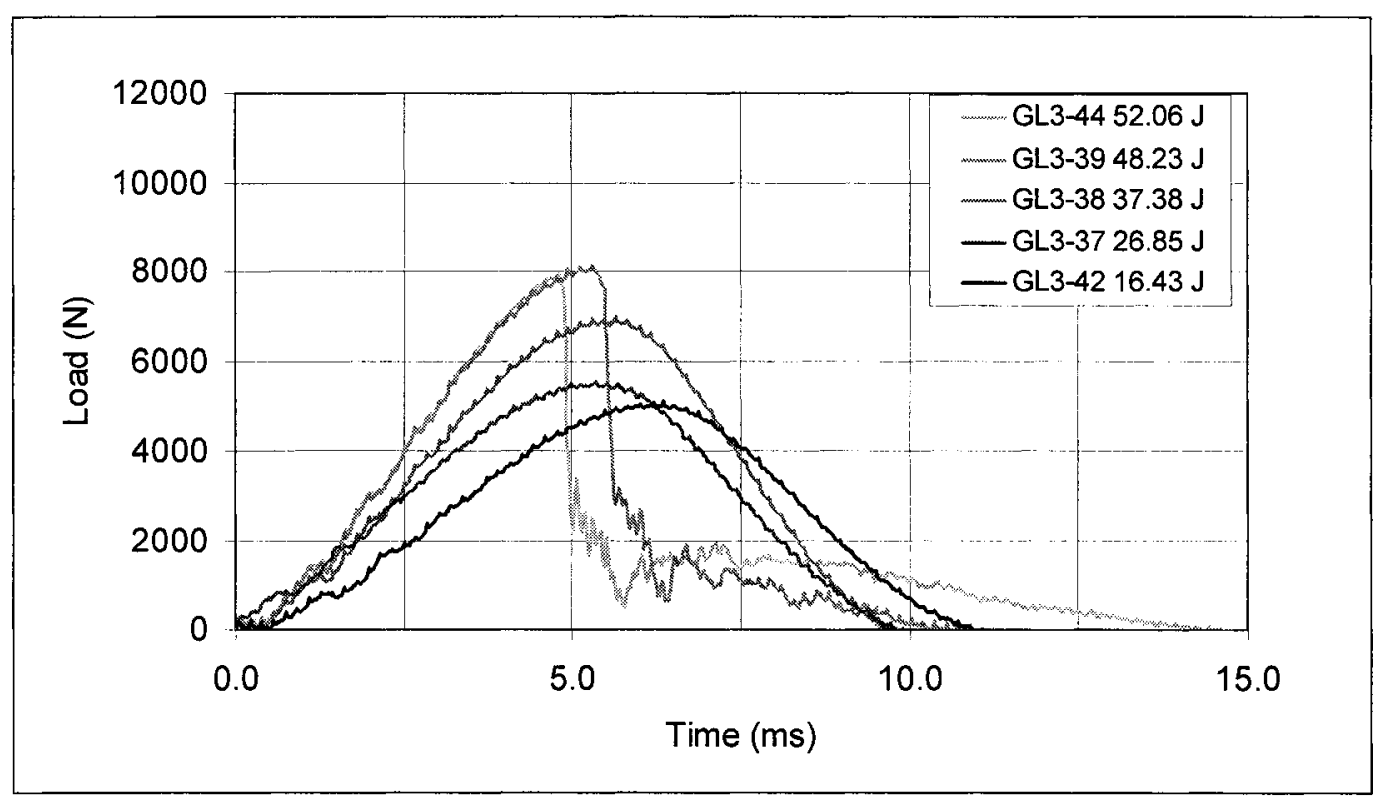

Figure 21: GLARE-3 sample load traces. 


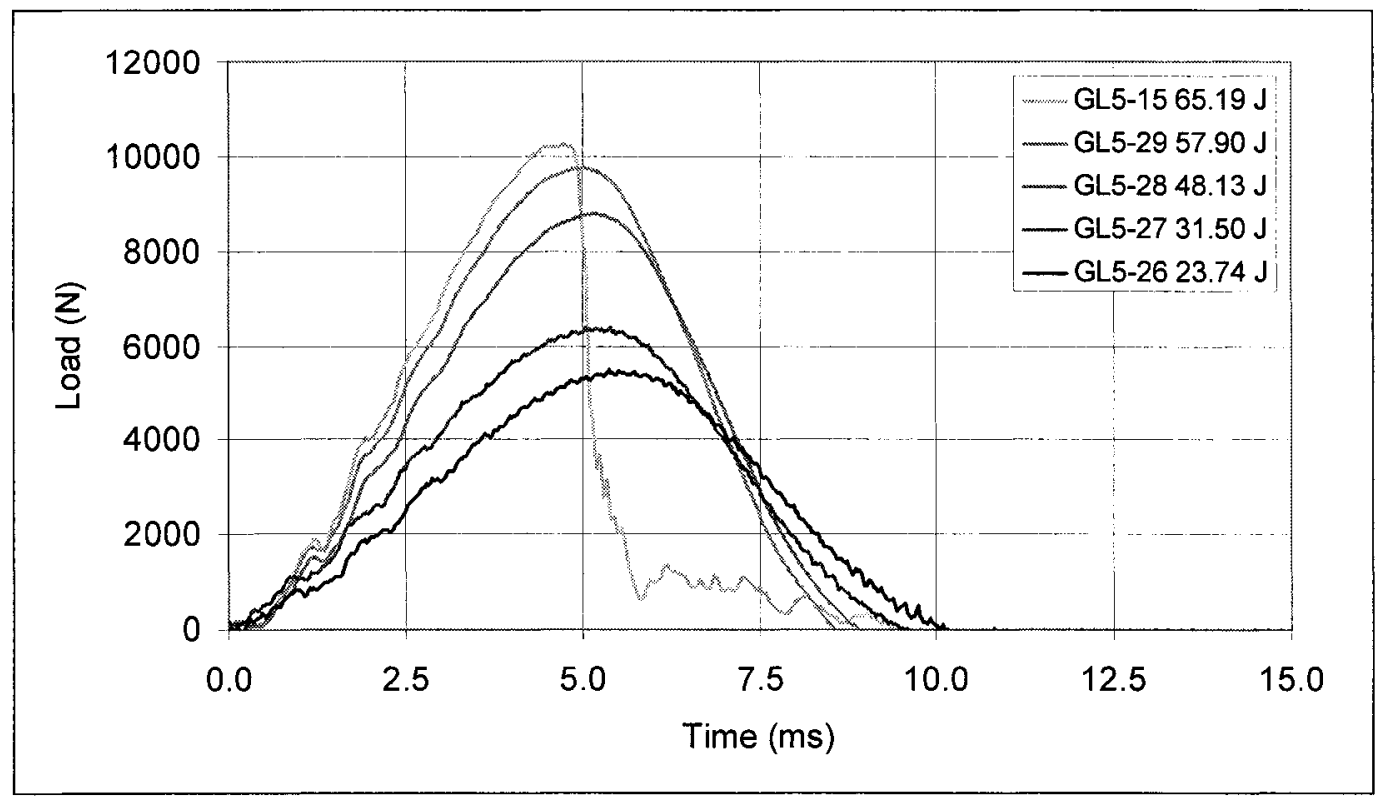

Figure 22: GLARE-5 sample load traces.

The impact force response of GLARE-4 panels shown in Figure 23 is significantly different. The load did not drop off as sharply or by as great a magnitude when the specimen was punctured (specimens GL4-24 and GL4-25). The impactinduced crack formed in a stable manner and dissipated the energy uniformly. This resulted in the crack geometry described previously. The crack always formed parallel to the major fibre direction (i.e. major loading direction). One implication of this behaviour is that a cyclic operating load would not cause the crack to grow unless the crack was at an angle to the load. This damage mode was also observed in thicker GLARE-4-3/2 laminates (Laliberté et al., 2001a). 


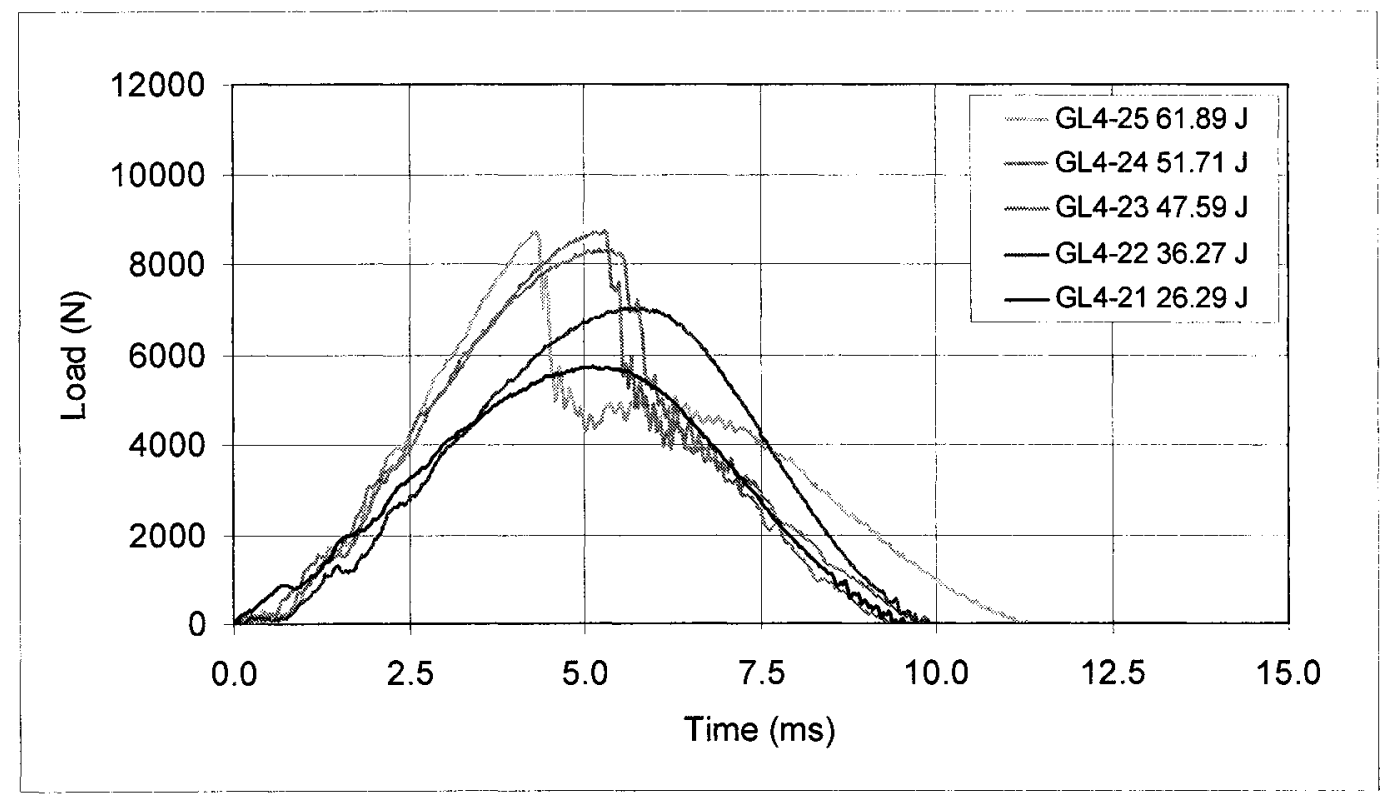

Figure 23: GLARE-4 sample load traces.

\section{Impact Strain Data}

Figure 24 shows the locations of the strain gauges relative to the impact point. All gauges were located $38.1 \mathrm{~mm}$ from the centre of the panel. Figures 25 to 27 show impact strain trace data for GLARE-3, GLARE-4 and GLARE-5. For most of the instrumented specimens strain data was collected from locations 1 and 4 and for several specimens data was collected at all locations. 


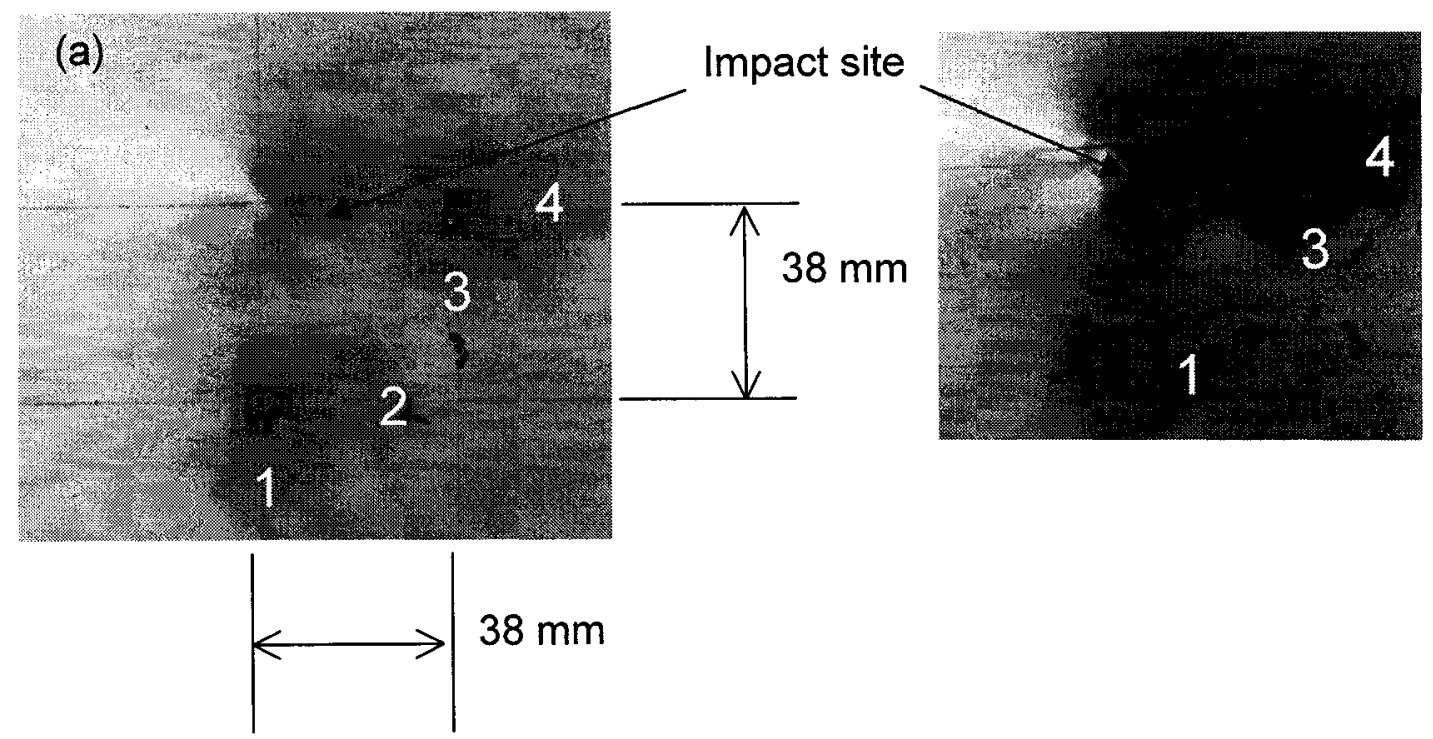

Figure 24: Strain gauge locations for four-gauge (a) and three-gauge (b) configurations.

The strain traces for GLARE-3 in Figure 25 show relatively smooth behaviour for energy levels below that required for penetration. At energy levels above the penetration threshold, the curves follow a shape similar to that of the load traces. The strain level dropped off sharply once the panel has been punctured. The peak strains measured by the gauges for each energy level are similar for both directions. This result is expected, since the GLARE- 3 has the same amount of fibres in the $0^{\circ}$ and $90^{\circ}$ directions. The curves shown in Figure 25 for the $16.4 \mathrm{~J}$ (GL3-36) energy level are skewed and smoothed because the strain gauge amplifiers were set with excessive noise filtering. 

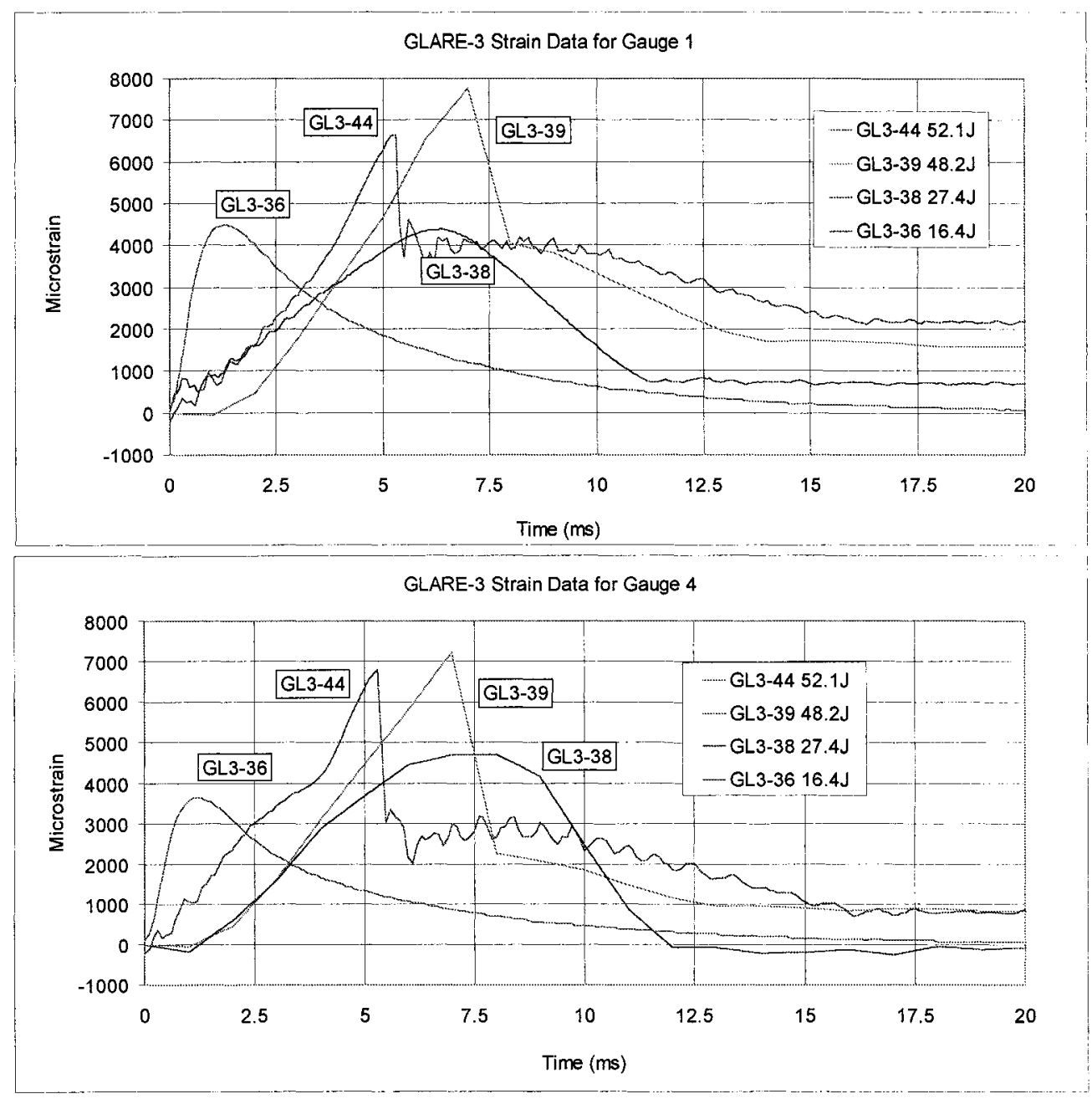

Figure 25: GLARE-3 impact strain traces for locations 1 and 4.

Unlike the GLARE-3 strain curves, the peak values for gauges 1 and 4 are significantly different for the GLARE-4 impacted panels. There are more than twice as many fibres oriented parallel to gauge $1\left(0^{\circ}\right)$ than there are for gauge $4\left(90^{\circ}\right)$. As can be seen in Figure 26, the strains recorded by gauge 4 are approximately twice as high as the values recorded by gauge 1 . This indicates that the panels are twice as stiff in the direction of gauge $1\left(0^{\circ}\right)$ than in the direction of gauge $4\left(90^{\circ}\right)$. After fracture, strain measured by gauge 1 drops off sharply. However, the strain measured by gauge 4 does not drop off after fracture. The fibres parallel to gauge 4 did not fracture and thus continued to carry loads when the crack formed. 

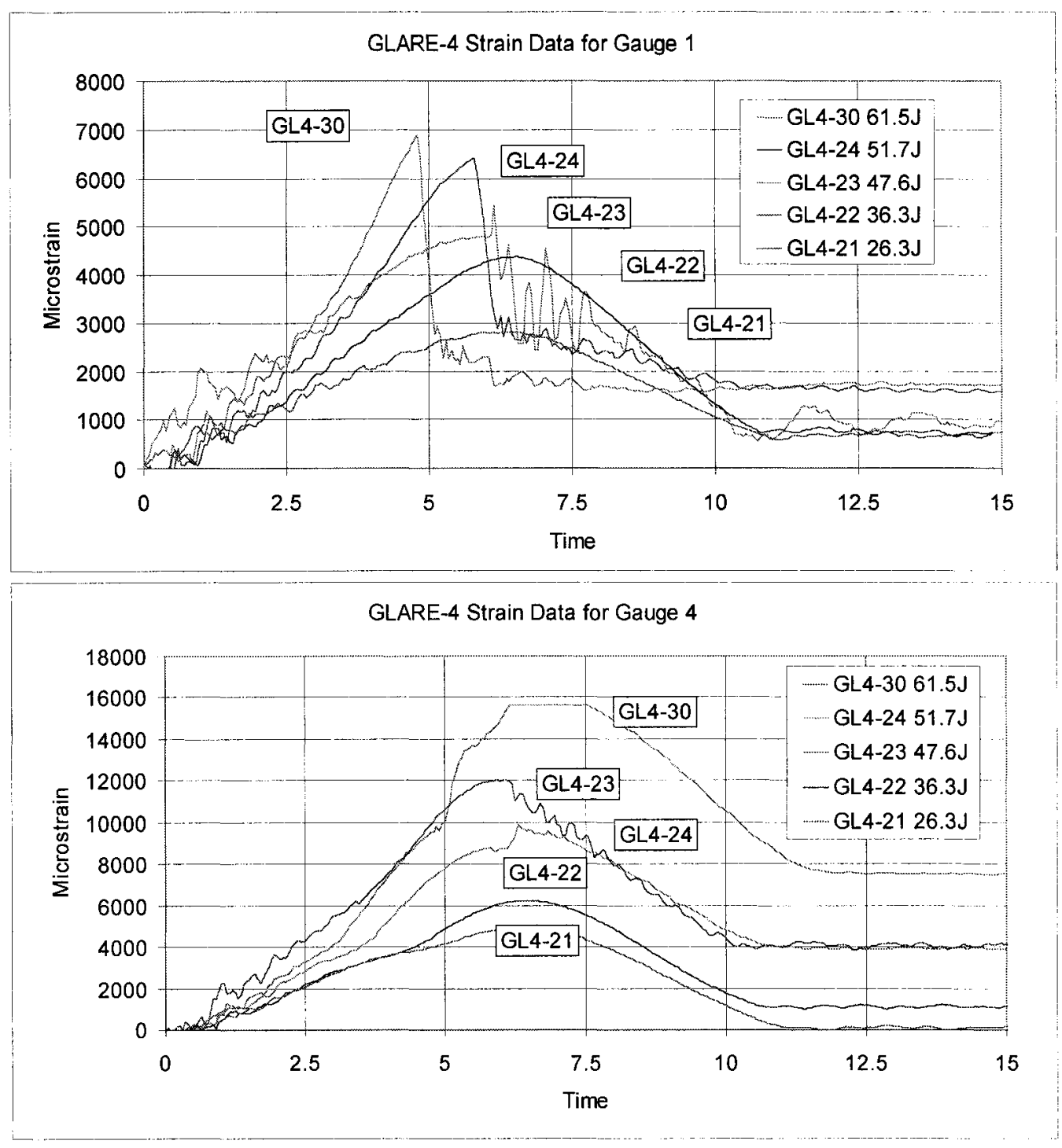

Figure 26: GLARE-4 impact strain traces for locations 1 and 4.

The GLARE-5-2/1 strain traces shown in Figure 27 are smooth, like the GLARE3 and GLARE-4 traces. Only one GLARE-5 specimen failed during this portion of the testing, GL5-30. The strain exhibited the same sharp drop at punctures as was observed for the GLARE-3 panels. The strain values for gauge 1 and gauge 4 are nearly identical for all the load traces as was also observed for GLARE-3. 

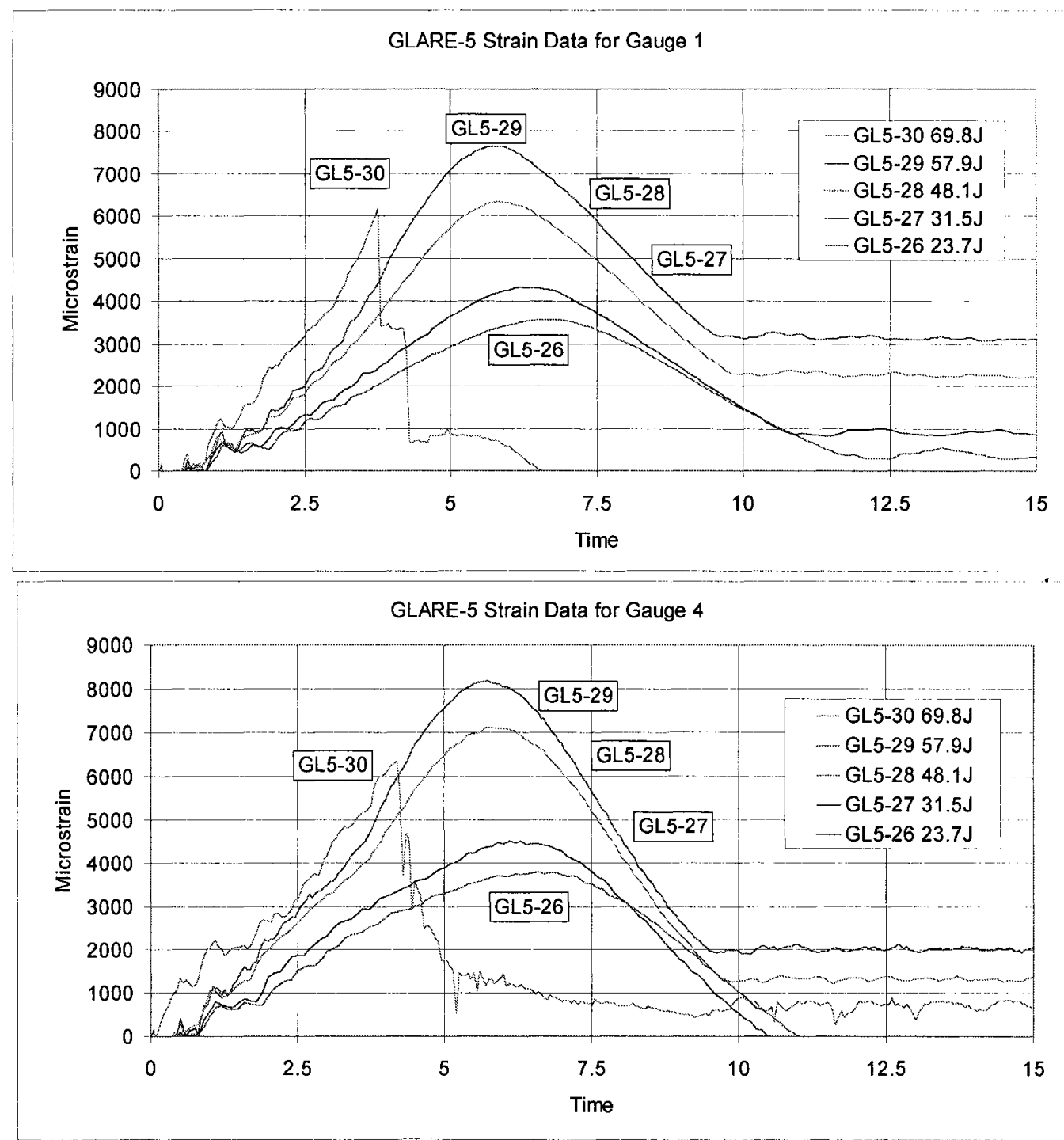

Figure 27: GLARE-5 impact strain traces for locations 1 and 4.

\section{Impact Damage Inspection}

Internal impact damage was visualized using the penetrant enhanced x-ray technique developed at IAR-SMPL (Fahr et al., 1999). Sample x-rays of the internal damage are shown in Figure 28. The effect of increasing impact energy is clearly shown in the figure as the extent of the damaged zone also increases. The small white circles in the centre of the images are the holes drilled to provide a penetrant path to the damage. 


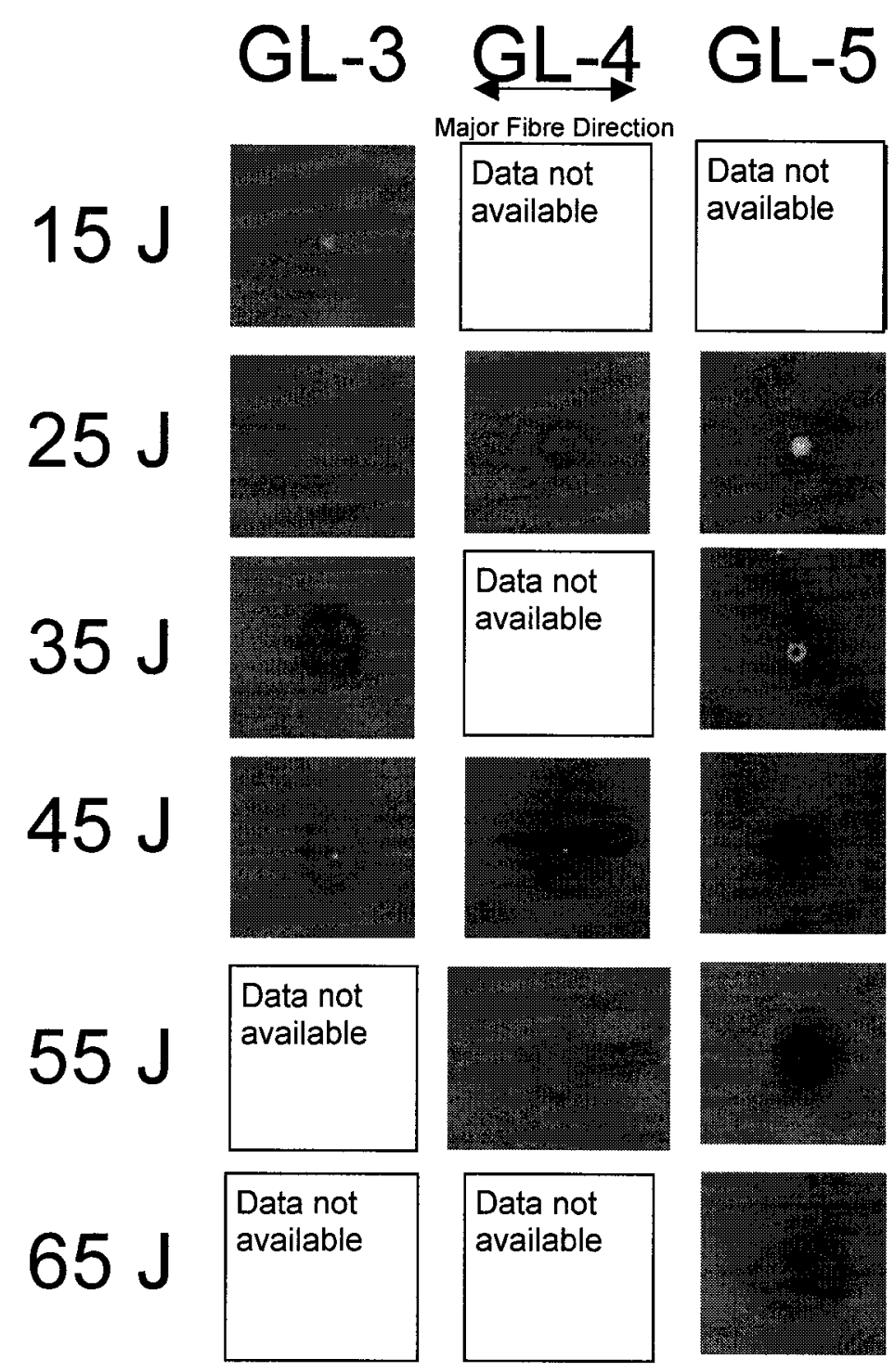

Figure 28: Penetrant enhanced x-ray images of impact damage.

Sample punctured panels were also photographed using visible light to record the damage patterns present in the panels as shown in Figures 29 to 31 . The damage observed for GLARE-3 and GLARE-5 was similar. Cracks that formed did not follow a preferential direction during growth. However, as stated earlier, the single crack formed in the GLARE-4 panels was always parallel to the major fibre direction. 


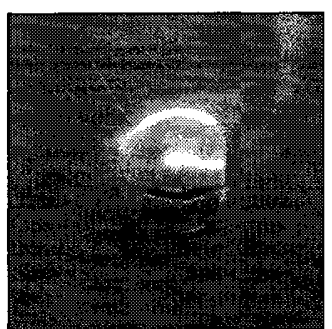

$47 \mathrm{~J}$

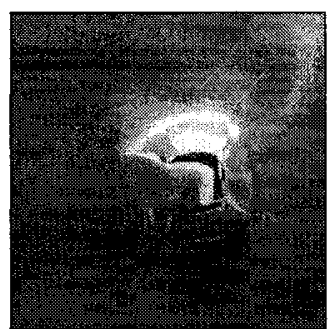

$52 \mathrm{~J}$

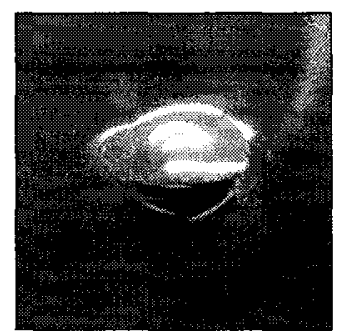

$52 \mathrm{~J}$

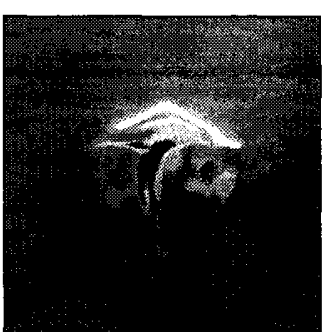

$51 \mathrm{~J}$

Figure 29: Puncture damage in GLARE-3.

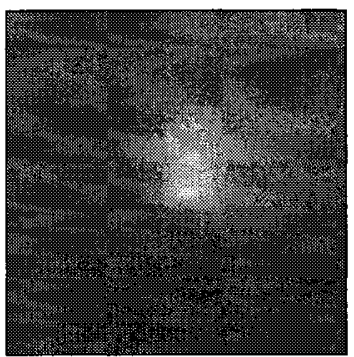

$47 \mathrm{~J}$

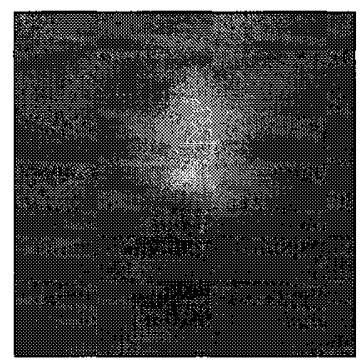

$54 \mathrm{~J}$

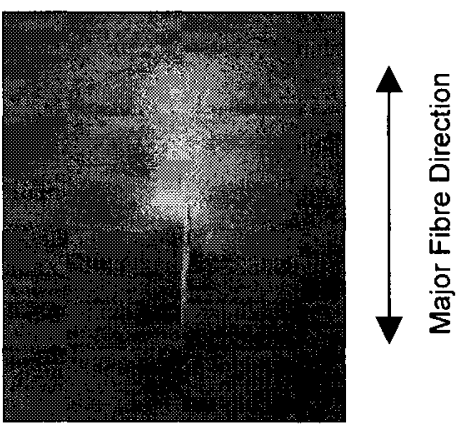

$61 \mathrm{~J}$

Figure 30: Puncture damage in GLARE-4.

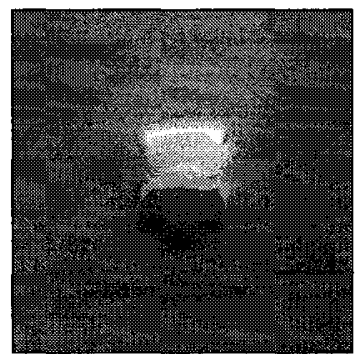

$58 \mathrm{~J}$

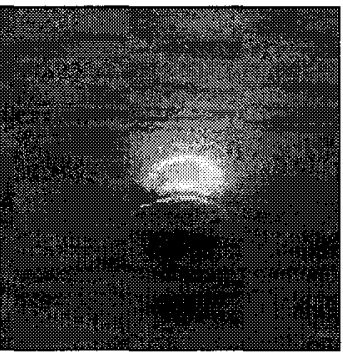

$67 \mathrm{~J}$

Figure 31: Puncture damage in GLARE-5.

Damage in the specimens was also examined using a destructive inspection technique. These results were used to determine the efficacy of the penetrant enhanced xray process. Seven impacted GLARE-3 and GLARE-5 specimens, which had previously been x-rayed, were cold-mounted in epoxy and then polished to reveal the internal damage. The specimens were cut from the impacted panels using a slow speed saw to 
prevent the introduction of additional damage. A further four GLARE-4 specimens that had not been $\mathrm{x}$-rayed were inspected. Figures 32 to 34 show unimpacted samples of GLARE-3, GLARE-4 and GLARE-5, provided for reference. As can be seen in these figures, there is no evidence of delamination from the mounting and polishing operations. Table 8 summarizes the delamination size measurements that were taken from both the $\mathrm{x}$ rays and the polished specimens.

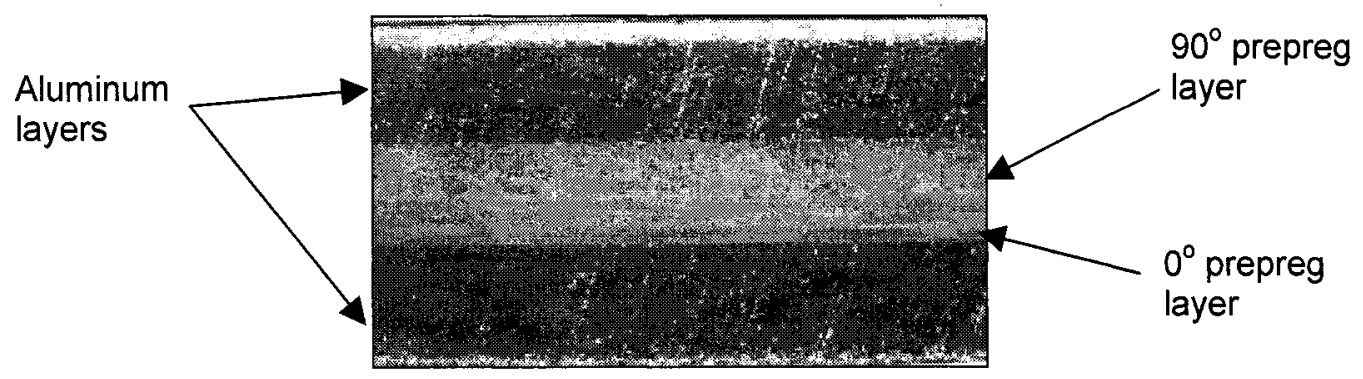

Figure 32: Cross-section of an unimpacted GLARE-3 specimen.

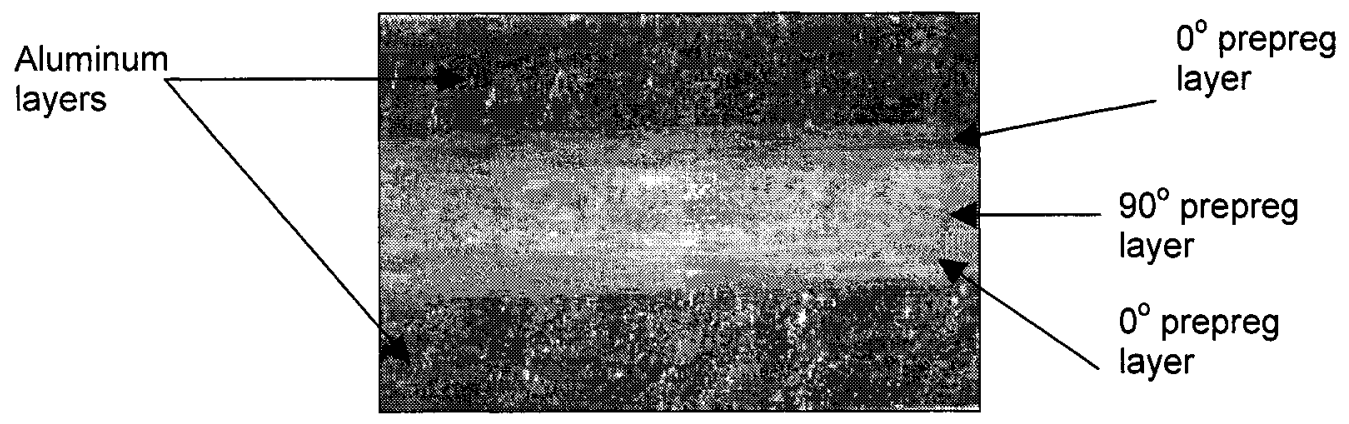

Figure 33: Cross-section of an unimpacted GLARE-4 specimen.

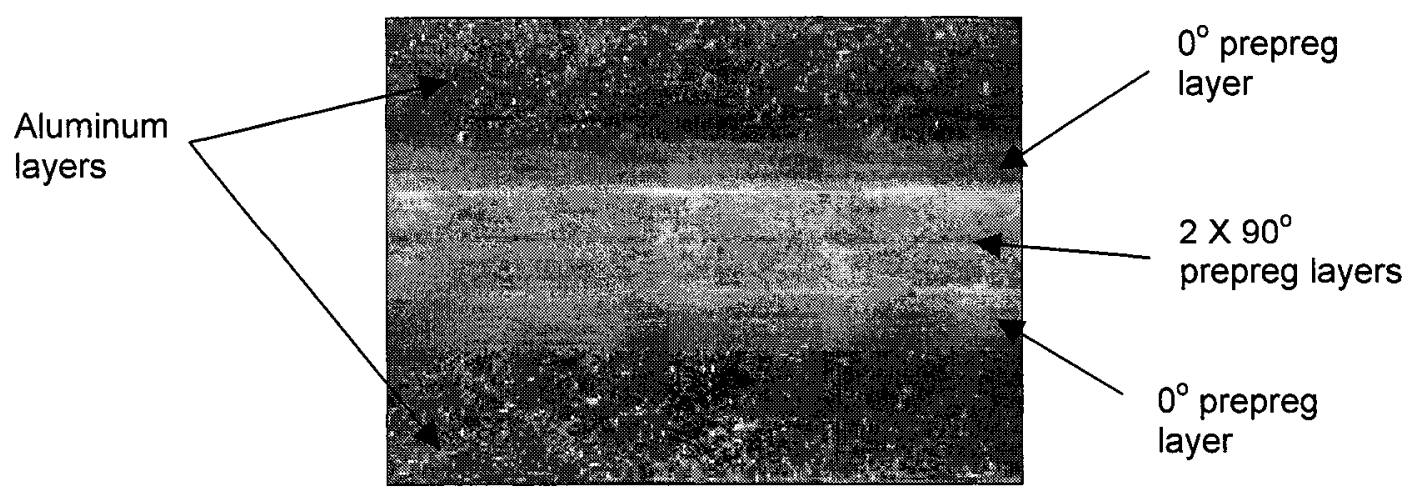

Figure 34: Cross-section of an unimpacted GLARE-5 specimen. 
Table 8: Delamination measurements from the $x$-rays and cross-sections.

\begin{tabular}{|c|c|c|c|c|}
\hline \multirow[t]{2}{*}{ Sample } & \multirow{2}{*}{$\begin{array}{c}E_{i m p} \\
(J)\end{array}$} & \multicolumn{2}{|c|}{$\begin{array}{l}\text { Delamination on x-ray } \\
(\mathrm{mm})\end{array}$} & \multirow{2}{*}{$\begin{array}{l}\text { Delamination in } 0^{\circ} \\
\text { direction }(\mathrm{mm})\end{array}$} \\
\hline & & $0^{\circ}$ & $90^{\circ}$ & \\
\hline $3-31$ & 15.73 & \multicolumn{2}{|c|}{ X-ray showed no delam } & 21.0 \\
\hline $3-32$ & 26.19 & \multicolumn{2}{|c|}{ X-ray showed no delam } & 19.5 \\
\hline $3-33$ & 36.42 & $\mathrm{~N} / \mathrm{A}$ & 16.0 & 27.5 \\
\hline $3-34$ & 46.72 & $\overline{N / A}$ & 17.0 & 28.0 \\
\hline $4-16$ & 26.44 & \multirow{4}{*}{\multicolumn{2}{|c|}{$\begin{array}{l}\text { No x-rays taken, information } \\
\text { provided for reference }\end{array}$}} & 8.0 \\
\hline $4-27$ & 36.88 & & & 10.5 \\
\hline $4-08$ & 47.03 & & & 16.0 \\
\hline $4-19$ & 53.27 & & & 21.0 \\
\hline $5-11$ & 25.82 & \multicolumn{2}{|c|}{ X-ray showed no delam } & 13.5 \\
\hline $5-12$ & 36.47 & \multicolumn{2}{|c|}{ X-ray showed no delam } & 22.0 \\
\hline $5-04$ & 57.74 & \multicolumn{2}{|c|}{ X-ray showed no delam } & 26.0 \\
\hline
\end{tabular}

Matrix cracking was observed in all of the specimens as indicated in Table 9. Delamination damage was also observed in all of the impacted specimens. However, as indicated in Table 9 this damage was not visible in all of the x-rays. Only GLARE-4 specimens containing through-cracks were selected for destructive inspection. It was in these specimens that fibre fracture was observed. No x-rays were available for these GLARE-4 specimens. Complete results of the cross-sectioning are presented in Appendix E. Typical results are shown below.

Table 9: Summary of observed damage in the cross-sectioned GLARE panels.

\begin{tabular}{|c|c|c|c|c|c|c|c|}
\hline \multirow{2}{*}{ Sample } & \multirow{2}{*}{$\frac{E_{i m p}}{(\mathrm{~J})}$} & \multicolumn{2}{|c|}{$\begin{array}{c}\text { Delamination } \\
\text { Visible }\end{array}$} & \multirow{2}{*}{$\begin{array}{l}\text { Matrix } \\
\text { Cracking } \\
\text { Visible }\end{array}$} & \multirow{2}{*}{$\begin{array}{c}\text { Fibre } \\
\text { Fracture } \\
\text { Visible }\end{array}$} & \multirow{2}{*}{$\begin{array}{c}\text { Fibre } \\
\text { Bridging } \\
\text { Visible }\end{array}$} & \multirow{2}{*}{$\begin{array}{c}\text { Aluminum } \\
\text { Fracture } \\
\text { Visible }\end{array}$} \\
\hline & & $\begin{array}{l}\ln X- \\
\text { Sect. }\end{array}$ & $\begin{array}{l}\operatorname{In} X- \\
\text { Ray }\end{array}$ & & & & \\
\hline $3-31$ & 15.73 & Yes & No & Yes & No & Yes & No \\
\hline $3-32$ & 26.19 & Yes & No & Yes & No & Yes & No \\
\hline $3-33$ & 36.42 & Yes & Yes & Yes & No & Yes & No \\
\hline 3-34 & 46.72 & Yes & Yes & Yes & No & Yes & No \\
\hline $4-16$ & 26.44 & Yes & $\mathrm{N} / \mathrm{A}$ & Yes & No & Yes & No \\
\hline $4-27$ & 36.88 & Yes & N/A & Yes & Yes & Yes & Yes \\
\hline $4-08$ & 47.03 & Yes & N/A & Yes & Yes & Yes & Yes \\
\hline 4-19 & 53.27 & Yes & N/A & Yes & Yes & Yes & Yes \\
\hline $5-11$ & 25.82 & Yes & No & Yes & No & Yes & No \\
\hline $5-12$ & 36.47 & Yes & No & Yes & No & Yes & No \\
\hline $5-04$ & 57.74 & Yes & Yes & Yes & No & Yes & No \\
\hline
\end{tabular}


Figures 35 to 46 show the typical damage modes observed in the cross-sections of the GLARE specimens. The x-ray of sample GLARE-3-31 (15.73 J), shown in Figure 35a, did not indicate the presence of delamination damage. However, the cross-section, Figure $35 \mathrm{~b}$, revealed delamination damage throughout the impacted area. The prepreg layers near the impacted surface of the panel were intact and adhering to the aluminum. This prevented adequate flow of the penetrant to the damage.
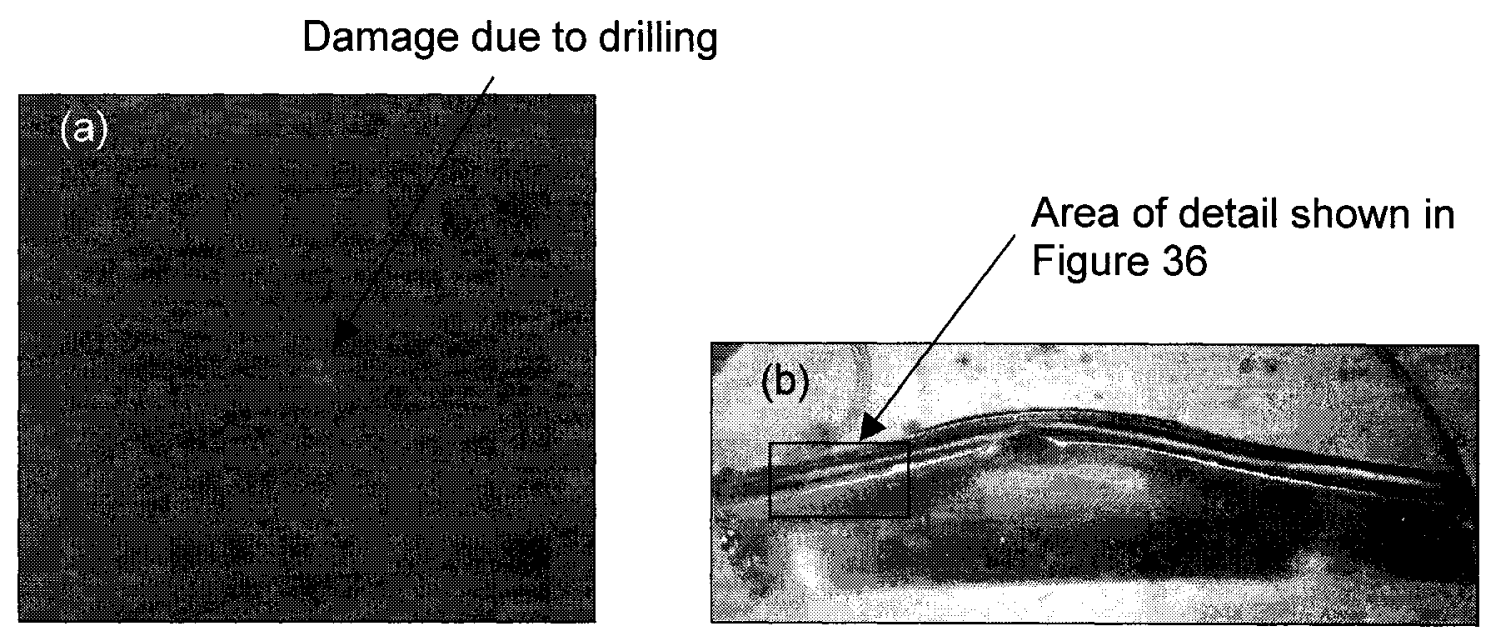

Figure 35: Sample GLARE-3-31 (15.73 J) (a) x-ray and (b) cross-section.

The GLARE-3-31 specimen shown in Figures 36 and 37 developed matrix cracking from the impact. Also, some of the individual fibres separated from the rest of the prepreg layer, with some adhesive left on the aluminum surface (Figure 36). This fibre-bridging was typical of impacted GLARE specimens.

Fibres

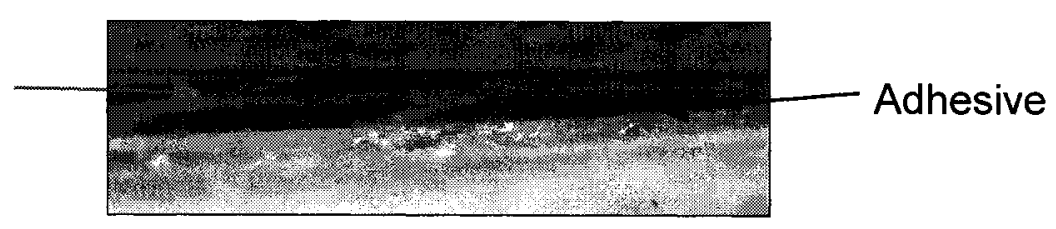

Figure 36: GLARE-3-31 (15.73 J) fibre delamination. 
The x-ray of GLARE-3-33 (36.42 J) in Figure 37a, revealed delamination damage from the impact. In this case the cross-section (Figure 37b) agreed with the x-ray. The sample also had matrix cracking (Figure 38) that is not visible in the x-ray. This cracking occurred between the fibres of the $90^{\circ}$ layer and no damage is apparent in the $0^{\circ}$ layer.
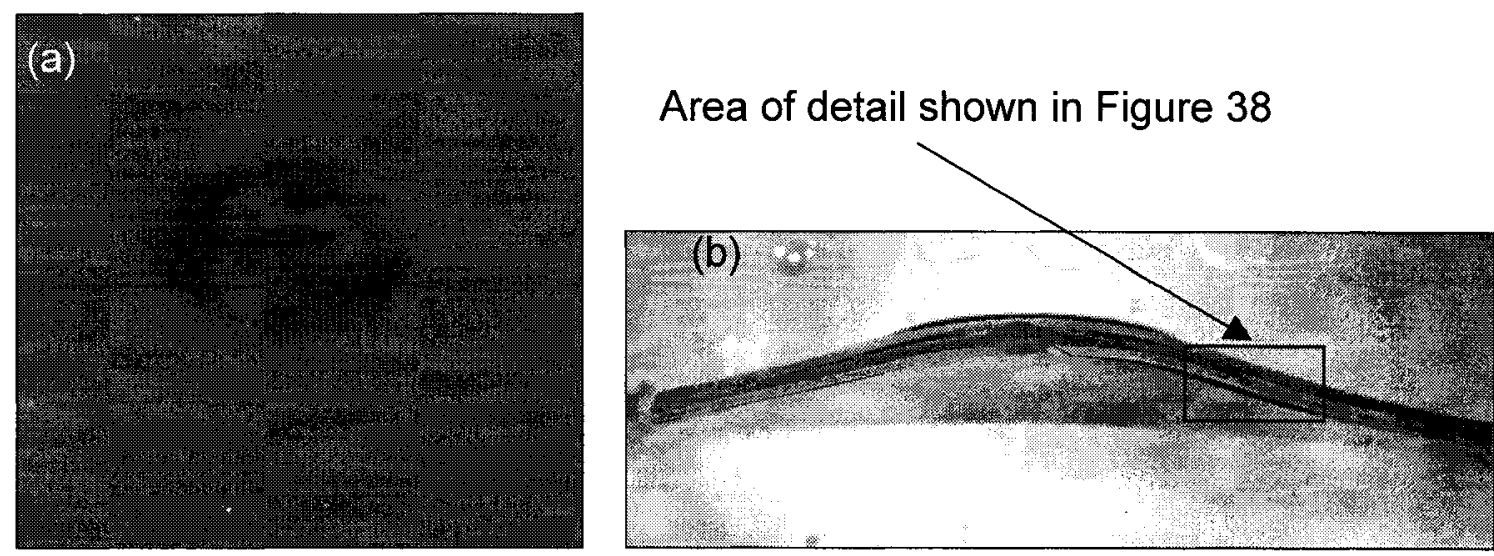

Figure 37: GLARE-3-33 (36.42 J) (a) x-ray and (b) cross-section.

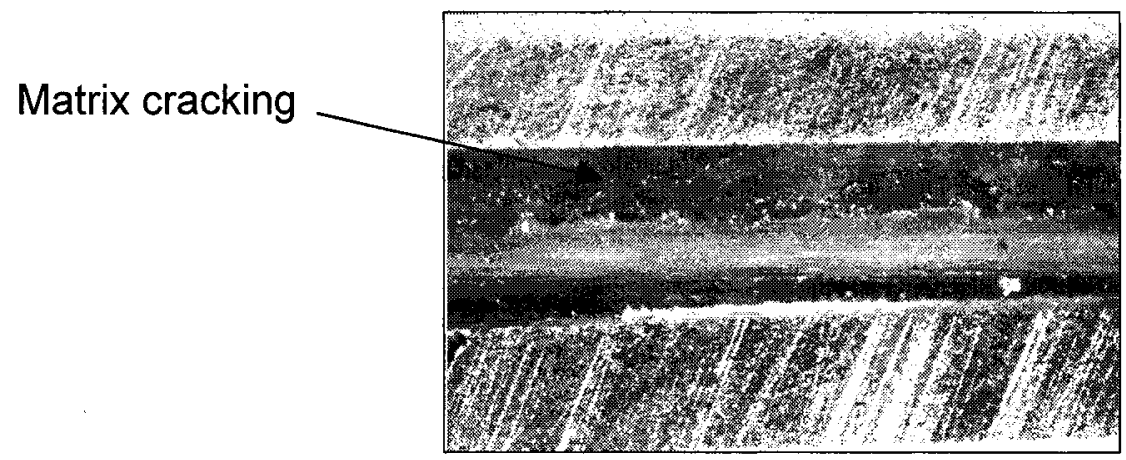

Figure 38: Matrix cracking in sample GLARE-3-33 in the $90^{\circ}$ layer.

Sample GLARE-4-08 (47.03 J) shown in Figure 39, was impacted with approximately the same energy level as sample GLARE-3-34 (46.72 J). However, the GLARE-4 sample developed a through-crack in addition to the delamination damage as shown in Figure 40. There was also matrix cracking as in GLARE-3-34. The fibres in the $0^{\circ}$ direction were fractured when the through-crack formed. The panel suffered extensive delamination (Figure 40a) and matrix cracking (Figures 40b and 41). The 
offset between the two halves of the crack occurred after the specimen was cut from the impacted panel; there was a release of stored elastic energy. It was not possible to determine if this release of energy could have increased the size of the delaminated region in these specimens.

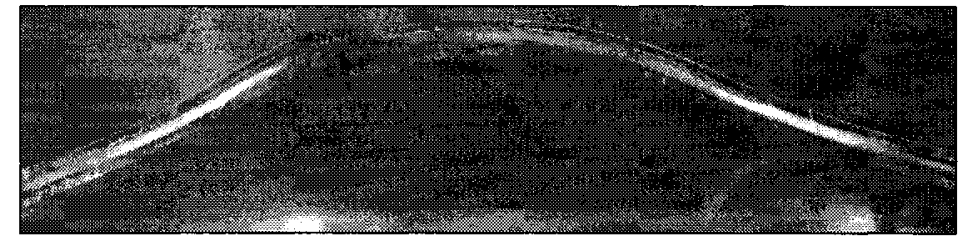

Figure 39: GLARE-4-08 (47.03 J) cross-section.

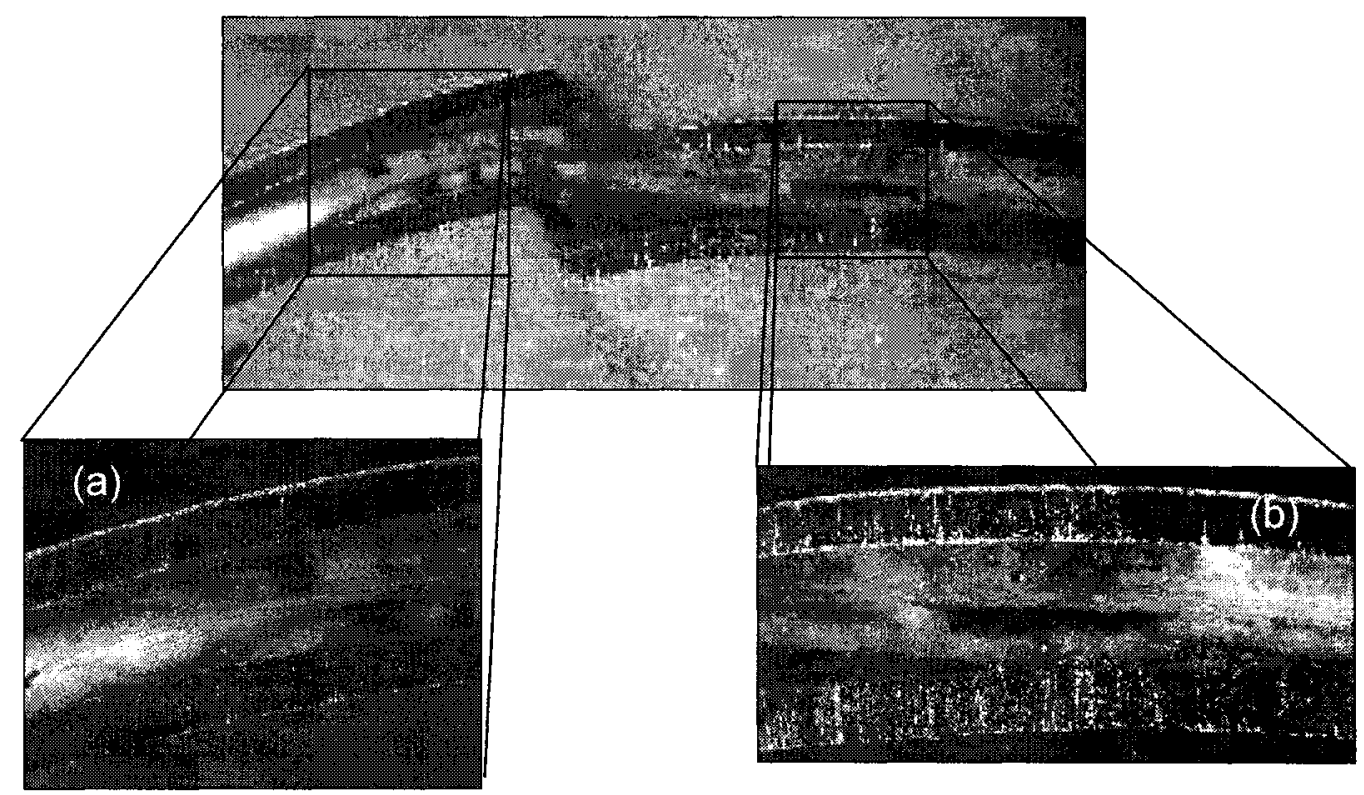

Figure 40: Through-crack in GLARE-4-08 (47.03 J).

Matrix cracking

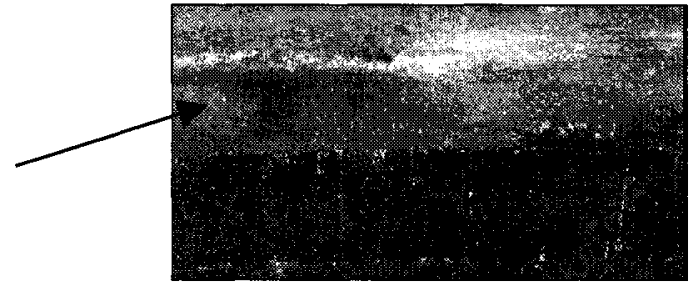

Figure 41: Matrix cracking in GLARE-4-08 (47.03 J) 
Sample GLARE-5-04 (57.74 J) showed considerable delamination damage (Figures $42 a$ and $42 b$ ). In this case the x-ray (Figure 42a) and the cross-section (Figure $42 \mathrm{~b})$ agreed. Where the dent starts to flatten out, there is significant delamination (Figures 43a and 43b). This sample also contained matrix cracks (Figure 44).
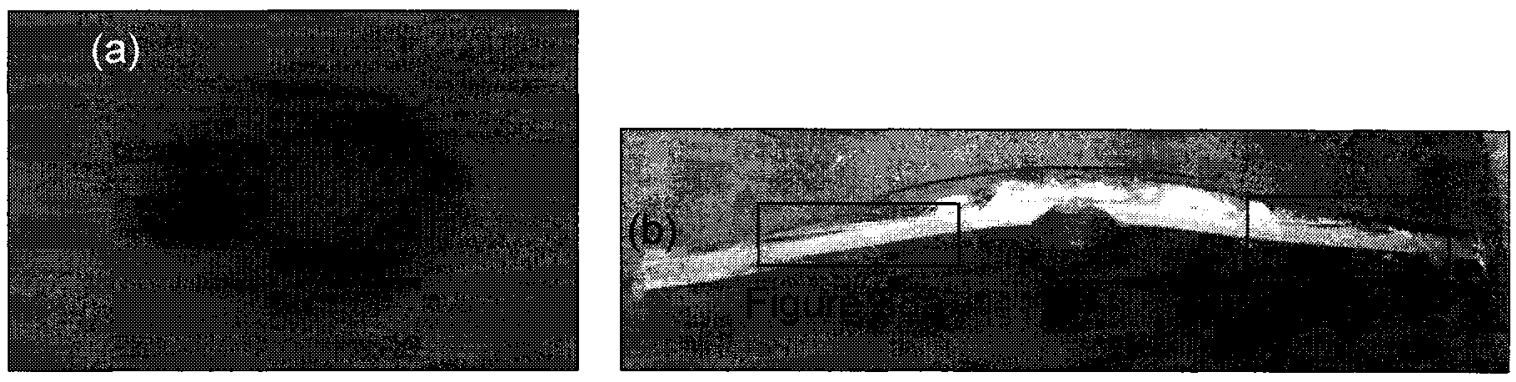

Figure 42: GLARE-5-04 (55 J) (a) x-ray and (b) cross-section.
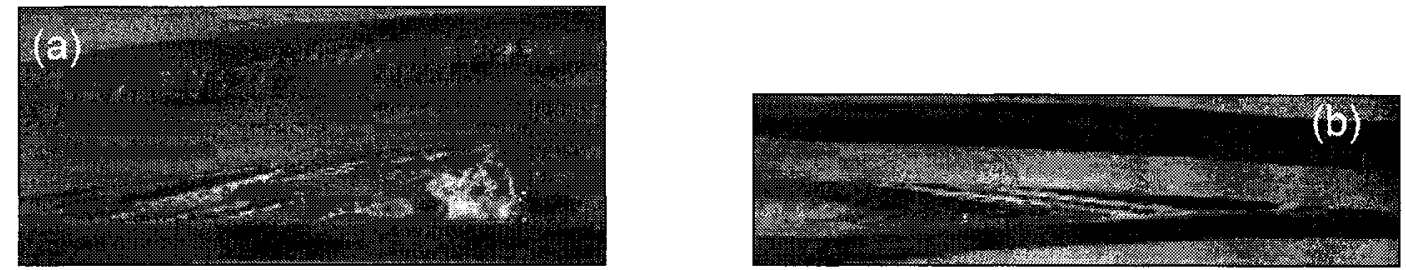

Figure 43: Delamination and fibre-bridging in GLARE-5-04 on (a) left and (b) right of dent.

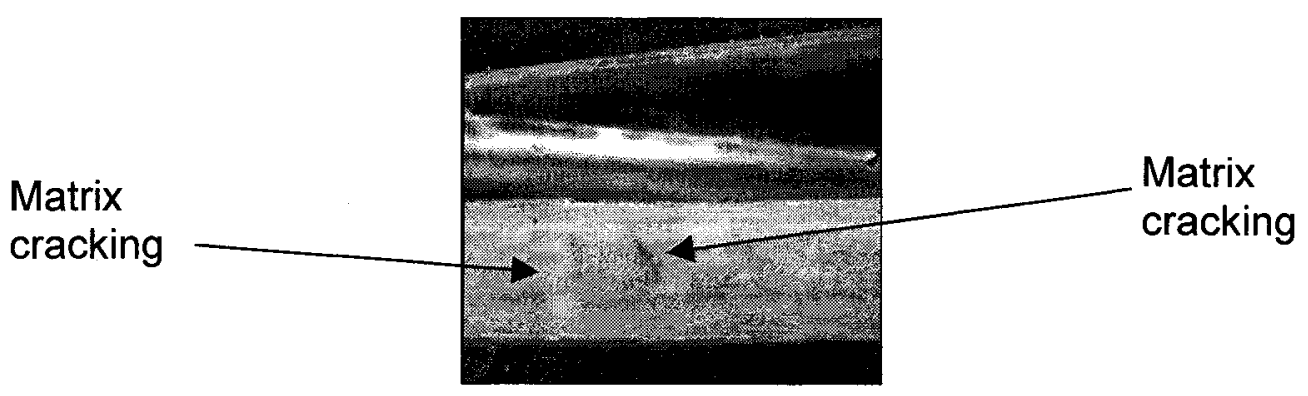

Figure 44: Matrix cracking in GLARE-5-04.

Sample GLARE-5-12 (36.47J) shown in Figure 45 developed delamination damage that was not apparent in the x-rays. This specimen provides a clear example of the type of matrix cracks that were typical of all the specimens that were inspected (Figure 46). 

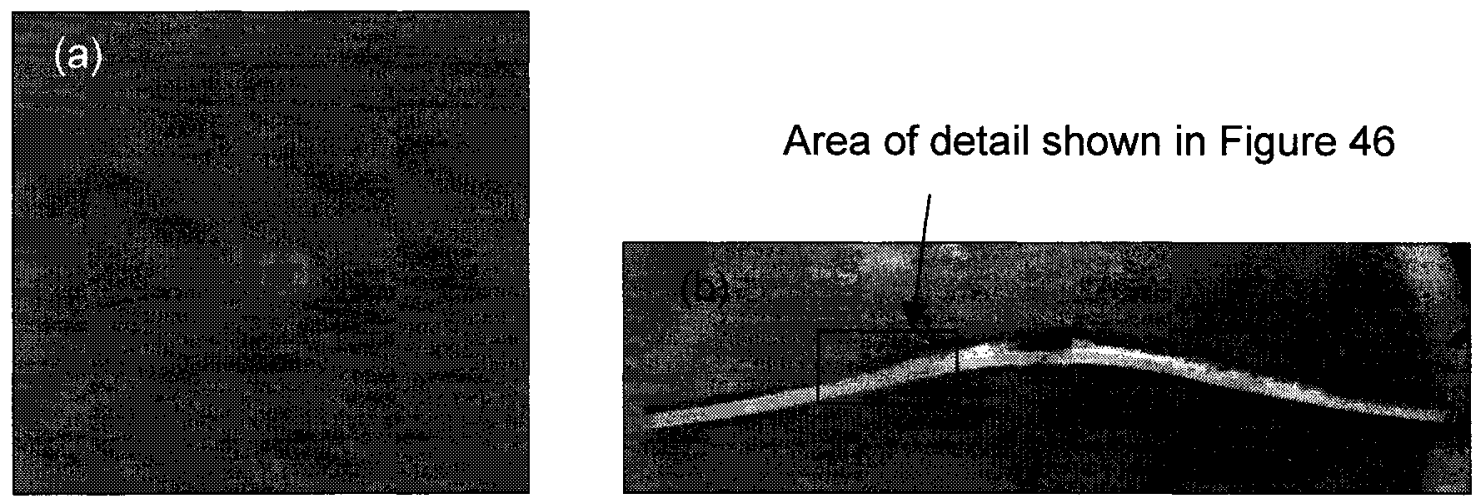

Figure 45: GLARE-5-12 (35 J) (a) x-ray and (b) cross-section.

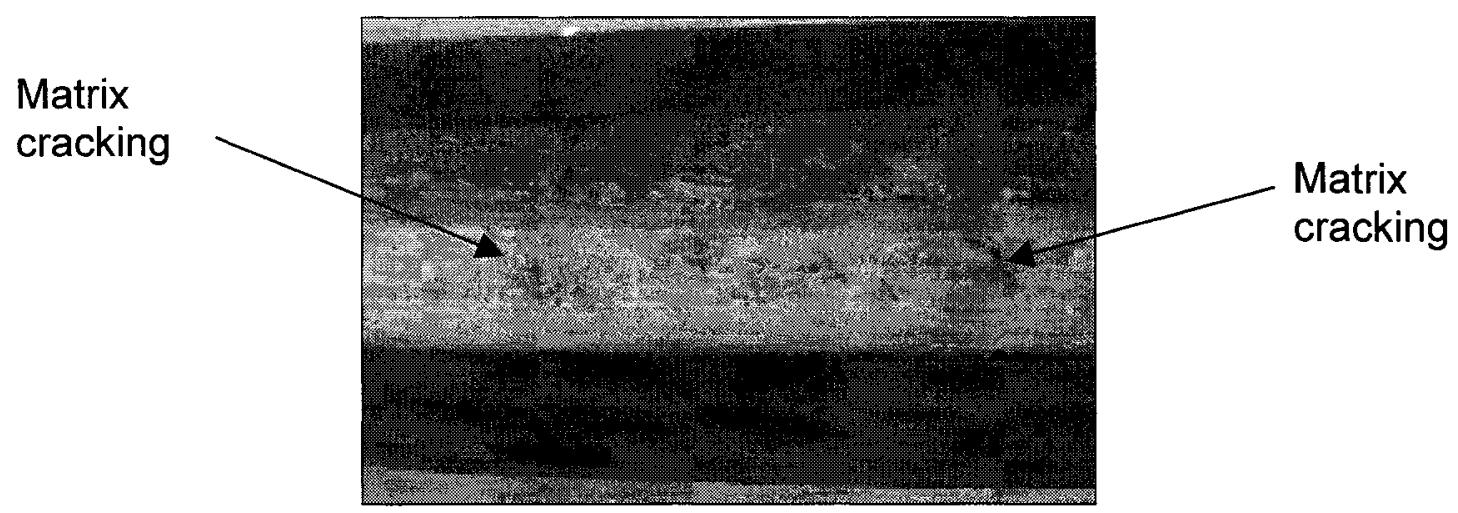

Figure 46: GLARE-5-12 matrix cracking and top $90^{\circ}$ layer adhesive failure.

Interlaminar shear forces between the aluminum and prepreg layers are responsible for the onset of delamination damage. As the impactor rebounds, the plastically deformed aluminum layers pull away from one another, causing regions of delamination to open up around the dent. Mode I delamination dominates the propagation of damage in impacted GLARE panels (Vlot, 1993b). However, initiation of the damage is controlled by mode II fracture strengths. This initial damage provides the initiation point for the development of mode I delamination. 
Table 10 summarizes the key results of the impact testing of GLARE-3, GLARE4 and GLARE-5. Aluminum 2024-T3 is not presented in this table since puncture did not occur in any of these specimens. The GLARE-5 laminates required higher energy for the onset of initial damage than the other two GLARE varieties. This material also showed higher energy for the onset of complete puncture.

A large number of experiments (162 total) were required to generate the impact characterization results described in the preceding sections. Future FML materials should be screened using impact simulations to reduce the testing requirements

Table 10: Summary of impact test results for GLARE laminates.

\begin{tabular}{|l||c|c|c|c|}
\cline { 3 - 5 } \multicolumn{1}{c|}{} & \multirow{2}{*}{ Units } & \multicolumn{3}{c|}{ Material } \\
\cline { 5 - 5 } \multicolumn{1}{c|}{} & & GLARE-3 & GLARE-4 & GLARE-5 \\
\hline Areal density & $\mathbf{k g} / \mathbf{m}^{2}$ & 2.168 & 2.418 & 2.688 \\
\hline Impact force to initiate internal damage & $\mathbf{k N}$ & 5.5 & 5.8 & 8.5 \\
\hline Energy of initiation of internal damage & $\mathrm{J}$ & 25 & 25 & 45 \\
\hline Peak strain at initiation (X-dir gauge 4) & $\mu \varepsilon$ & 4500 & 4500 & 7050 \\
\hline Peak strain at initiation (Y-dir gauge 1) & $\mu \varepsilon$ & 4200 & 2800 & 6400 \\
\hline Impact force to initiate partial puncture & $\mathbf{k N}$ & & 7.1 & 9.6 \\
\hline Energy of initiation of partial puncture & $\mathrm{J}$ & \multirow{2}{*}{ No partial } & 35 & 58 \\
\hline Peak strain at initiation (X-dir gauge 4) & $\mu \varepsilon$ & puncture & 12000 & 8200 \\
\hline Peak strain at initiation (Y-dir gauge 1) & $\mu \varepsilon$ & & 5400 & 7800 \\
\hline Impact force for complete puncture & $\mathbf{k N}$ & 7.9 & 8.2 & 10.3 \\
\hline Initiation energy for complete puncture & $\mathbf{J}$ & 46 & 51 & 65 \\
\hline Peak strain at puncture (X-dir gauge 4) & $\mu \varepsilon$ & 4800 & 10000 & 6300 \\
\hline Peak strain at puncture (Y-dir gauge 1) & $\mu \varepsilon$ & 7800 & 6500 & 6300 \\
\hline
\end{tabular}




\section{CHAPTER 3}

\section{FML IMPACT MODELLING}

\section{METHODOLOGY}

Based on the results of the low-velocity impact tests described in the previous Chapter, it was decided to develop a methodology to model low-velocity impacts on FML panels. This Chapter presents an overview of the planned development of this methodology. A review of modelling techniques that have previously been applied to FMLs is first presented. This review revealed the capabilities and limitations of existing modelling techniques. Next, a conceptual overview of the proposed modelling methodology and its components is provided in Section 3.2. Finally, a description of some of the key features of the explicit finite element code LS-DYNA is presented in Section 3.3. This code was chosen to model low-velocity impact in the present work. Subsequent Chapters will describe the implementation of each component of the methodology, culminating with its application to predict the impact response of GLARE presented in Chapter 7. 


\subsection{Review of Modelling of FMLs}

Efforts have been made by researchers over the last decade to model the impact response of FMLs using either analytical or finite element methods. Table 11 presents a summary of recent modelling efforts and also lists their applications, capabilities and limitations. Several of the more important and potentially useful methods will be discussed in greater detail. However, none of these models is comprehensive. For example, some do not account for delamination while others neglect the loss of stiffness through the formation of microcracks in the prepreg. The experimental results presented in the previous Chapter showed that both of these damage modes occur in impacted FML panels. Therefore, a representation of these damage modes is required to predict the impact response of FMLs. Finally, a review of delamination prediction models for FMLs and composites will be presented. These delamination methods were not all developed specifically for impact simulations.

Table 11: Summary of modelling techniques applied to FMLs.

\begin{tabular}{|l|l|l|l|l|}
\hline \multicolumn{1}{|c|}{ Reference } & \multicolumn{1}{c|}{ Application } & \multicolumn{1}{c|}{ Type } & \multicolumn{1}{c|}{ Capabilities } & \multicolumn{1}{c|}{ Limitations } \\
\hline $\begin{array}{l}\text { Hurez et al., } \\
1993\end{array}$ & $\begin{array}{l}\text { Static loading } \\
\text { simulations }\end{array}$ & FEA & $\begin{array}{l}\text { Damage in the } \\
\text { prepreg, }\end{array}$ & No delamination \\
\hline $\begin{array}{l}\text { Hashagen et } \\
\text { al., 1995 }\end{array}$ & $\begin{array}{l}\text { Static loading } \\
\text { simulations }\end{array}$ & FEA & $\begin{array}{l}\text { Elastic-plastic } \\
\text { behaviour of aluminum }\end{array}$ & No delamination \\
\hline $\begin{array}{l}\text { Hashagen et } \\
\text { al., 1999 }\end{array}$ & $\begin{array}{l}\text { Delamination } \\
\text { fatigue }\end{array}$ & FEA & Delamination included & $\begin{array}{l}\text { No plasticity, } \\
\text { prepreg elastic up } \\
\text { until failure, }\end{array}$ \\
\hline Vlot, 1998b & Impact & $\begin{array}{l}\text { Non-linear } \\
\text { Analytical }\end{array}$ & $\begin{array}{l}\text { Elastic plastic } \\
\text { behaviour of aluminum }\end{array}$ & $\begin{array}{l}\text { No delamination, } \\
\text { assumed elastic } \\
\text { prepreg }\end{array}$ \\
\hline Vlot, 1993a & Impact & $\begin{array}{l}\text { Linear } \\
\text { Analytical }\end{array}$ & $\begin{array}{l}\text { Can model contact } \\
\text { forces }\end{array}$ & $\begin{array}{l}\text { No delamination, } \\
\text { assumed elastic } \\
\text { prepreg }\end{array}$ \\
\hline Sun et al., 1993 & Impact & $\begin{array}{l}\text { Non-linear } \\
\text { Analytical }\end{array}$ & $\begin{array}{l}\text { Can model contact } \\
\text { forces, can model dent } \\
\text { depth }\end{array}$ & $\begin{array}{l}\text { No delamination, } \\
\text { assumed elastic } \\
\text { prepreg }\end{array}$ \\
\hline
\end{tabular}




\subsubsection{Finite Element Modelling of FMLs}

In 1993, Hurez et al. described an elastic-plastic model incorporating damage for static loading cases. A membrane-bending finite element was employed that included transverse stresses. Each layer in the laminate was then modelled either as elastic-plastic with strain hardening (metal) or transversely isotropic with damage (prepreg). The behaviour of the aluminum layers was modelled using the following threshold for the onset of plasticity:

$$
\bar{\sigma}-\sigma_{y c u r r e n t}=0
$$

where $\bar{\sigma}=\sqrt{3 J_{2}}$ is the equivalent von Mises stress and ( $\left.\sigma_{y \text { current }}\right)$ is the current yield stress taking into account isotropic strain hardening. Incorporating a damage prediction for the prepreg layers into the model is somewhat more problematic. Hurez et al. based their approach upon the elementary ply damage mechanics theory developed by Ladevèze and Le Dantec (1992) for use with composite materials. This theory is described in greater detail in Section 6.1 covering continuum damage mechanics for FMLs.

Hashagen et al. (1995) described a geometrically and materially non-linear thickshell element that was developed for FMLs. This element employs 16 nodes at the top and bottom surfaces. The von Mises isotropic yield criterion is employed for the aluminum layers. The prepreg layers are modelled using the Hoffman yield criterion. This criterion employs terms that vary linearly with the stress so that differences in tensile and compressive response can be properly described. Several benchmark tests from literature were modelled by Hashagen et al. (1995) using their shell element. The benchmark results agreed with those obtained using conventional thin-shell elements. 
The main shortfall of the previous two models is the lack of delamination damage in the predictions. Hashagen et al. (1999) described a technique for modelling delamination in spliced FML panels. Spliced FMLs have been designed to reduce the requirement for mechanically fastened (i.e. riveted) joints in large diameter aircraft fuselages. The splice (Figure 47) overcomes the limitations placed on the production of FML panels by the available width of aluminum sheets. The aluminum layers are reinforced with continuous prepreg layers, resulting in an efficient structural joint.

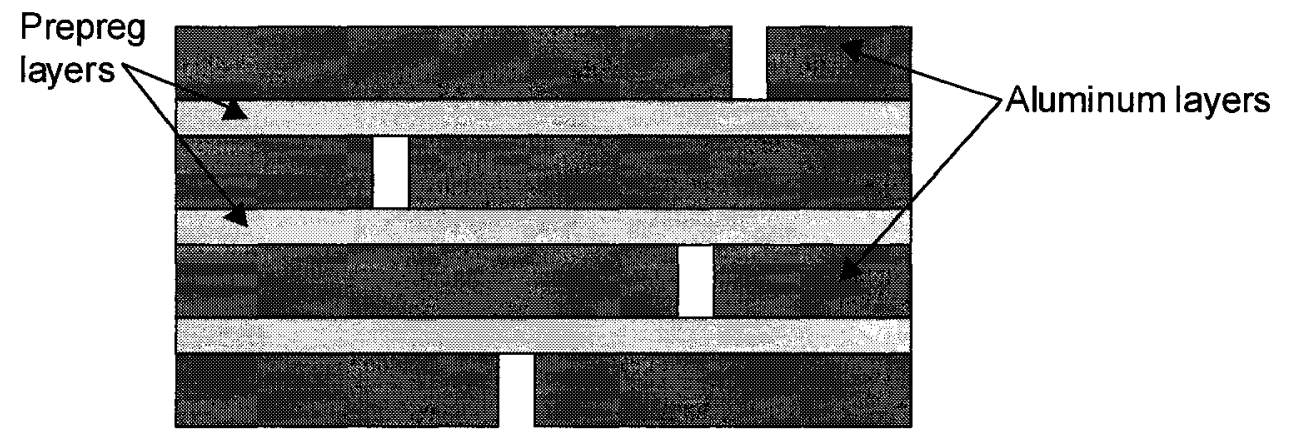

Figure 47: A basic FML splice concept (not to scale).

Hashagen et al. (1999) used this model to predict delamination growth in spliced panels tested previously by de Vries et al. (1999). Once again, Hashagen et al. used thick-shell elements to model the laminates. A layer of interface elements was inserted between the layers of aluminum and prepreg. A Hoffman-like delamination model was introduced that used a yield function to predict the failure of the interface. This approach assumed propagation of delamination from an initial flaw. It was also assumed that the delamination was dominated by mode II crack growth. With this assumption, the numerical results agreed well with experiments for double-sided splice joints. However, there were some differences between the results for single-sided, asymmetrical splices. There was significant out-of-plane bending experienced by this configuration. This 
resulted in mixed-mode delamination where the mode I contribution was no longer negligible. Due to this geometrically non-linear behaviour, the model could not accurately predict delamination growth in the single-sided splice.

\subsubsection{Analytical Modelling of Impact on FMLs}

In addition to finite element methods there are also several closed-form analytical techniques that have been applied to impact in FMLs. Vlot (1996 and 1998) developed a model to describe the deflection of a GLARE panel under low-velocity impact loading. One of the key assumptions was that the deflection-energy curves are approximately equal for quasi-static and low-velocity impact loading. At low strain levels the deformation of the panel is dominated by bending. Higher order non-linear terms that relate to the stretching of the material must be taken into account for larger deflections. In Equations 6 to 8, Vlot employed the Von Kármán relationships for the assumption of small strains and moderate deflection levels in a circular plate.

$$
\begin{aligned}
& \varepsilon_{r}=\frac{d u}{d r}+\frac{1}{2}\left[\left(\frac{d u}{d r}\right)^{2}+\left(\frac{d w}{d r}\right)^{2}\right]-z \frac{d^{2} w}{d r^{2}} \\
& \varepsilon_{\theta}=\frac{u}{r}-\frac{z}{r} \frac{d w}{d r} \\
& \gamma_{r \theta}=0
\end{aligned}
$$

where, $w$ is the general out-of-plane deflection of the circular area, $u$ is the in-plane displacement, and $r, z$ and $\theta$ are the radial, out-of-plane and angular directions. The deflection shape function that Vlot employed, Equation 9, satisfies the fully clamped boundary conditions of the circular impacted area. 


$$
\xi=\left(1-\eta^{2}\right)^{2}\left(1-1.2 \eta+1.2 \eta^{2}\right)
$$

where $\xi=w / w_{o}$ is the normalized deflection and $\eta=r / a$ is the normalized relative position from the centre of the panel. In this case $w_{o}$ is the central deflection of the panel. Now, it is shown in Equations 10 to 12 that the Von Kármán relations can be rewritten in terms of normalized co-ordinates.

$$
\begin{aligned}
& \varepsilon_{r}=\frac{w_{0}^{2}}{2 a^{2}}\left(\frac{d \xi}{d \eta}\right)^{2}-\frac{z w_{0}}{a^{2}} \frac{d^{2} \xi}{d \eta^{2}} \\
& \varepsilon_{\theta}=-\frac{z w_{0}}{a^{2} \eta}\left(\frac{d \xi}{d \eta}\right) \\
& \gamma_{r \theta}=0
\end{aligned}
$$

where, $\varepsilon_{r}$ is the in-plane strain in the radial direction, $\varepsilon_{\theta}$ is the in-plane strain in the circumferential direction and $\gamma_{r \theta}$ is the in-plane shear strain. Vlot employed the effective strain and a bi-linear stress-strain curve for the aluminum alloy to calculate the elasticplastic energy by integrating over the aluminum volume of the circular plate. The effective strain in the metallic layers is given as:

$$
\bar{\varepsilon}^{2}=\frac{4}{3}\left[\varepsilon_{r}^{2}+\varepsilon_{\theta}^{2}+\varepsilon_{r} \varepsilon_{\theta}\right]
$$

The elastic-plastic energy stored in the metallic volume is given in two parts, the energy from elastic deformation and plastic deformation:

$$
\begin{aligned}
& d U_{e}=\frac{1}{2} E \bar{\varepsilon}^{2} \text { for } \bar{\varepsilon}<\frac{\sigma_{y}}{E} \\
& d U_{p}=\frac{1}{2} \alpha \bar{\varepsilon}^{2}+\left[1-\frac{1}{2} \frac{\alpha}{E}\right] \frac{\sigma_{y}}{E}-\frac{1}{2} \frac{\sigma_{y}^{2}}{E}\left[1-\frac{\alpha}{E}\right] \text { for } \bar{\varepsilon}>\frac{\sigma_{\mathrm{y}}}{\mathrm{E}}
\end{aligned}
$$


In Equation $14 \alpha$ is the central deflection of the panel. The total strain energy in the metal layers is obtained by employing the integral shown in Equation 15. The integration is then performed through a numerical summation of the strain energy for small elements over the entire plate. First, the mean radial and tangential strains are calculated from Equations 10 to 12 with the known shape of the dent.

$$
U_{p}=\int_{z} \int_{r} d U_{p} 2 \pi r d r d z
$$

Next, the strain in the prepreg layers is given in Equation 16.

$$
\varepsilon_{f}=\varepsilon_{r} \cos ^{2} \theta+\varepsilon_{\theta} \sin ^{2} \theta
$$

The key assumptions that Vlot (1996) made at this point are that the prepreg layers deform elastically and that all of the strain energy is stored in the glass-fibres. The elastic deformation assumption appeared to be valid since the fibres yield at a significantly higher stress level than the aluminum. The resulting integration required to find the total strain energy stored in the fibre layers is shown in Equation 17.

$$
\begin{aligned}
U_{f} & =\int_{V} \iint d U_{f} r d \theta d z=\int_{V} \iint \frac{1}{2} E_{f}\left[\varepsilon_{f}(\theta)\right]^{2} r d \theta d z \\
& =\int_{A} \int \frac{1}{2} \pi E_{f}\left[\frac{3}{4} \varepsilon_{r}^{2}+\varepsilon_{r} \varepsilon_{\theta}+\frac{3}{4} \varepsilon_{\theta}^{2}\right] r d r d z
\end{aligned}
$$

where $E_{f}$ is the modulus of the prepreg layer in the fibre direction.

The integral in Equation 17 is computed in the same manner as that shown in Equation 15 for the aluminum layers. The contact force between the impactor and the panel is then found to be:

$$
F=\frac{d U}{d w_{0}} \approx \frac{U\left(w_{0}+\delta\right)-U\left(w_{0}\right)}{\delta}
$$

where $U=U_{p}+U_{f}$ and $\delta$ is a small value, e.g., $0.0001 w_{0}$ as suggested by Vlot (1998). No explanation for the selection of this value was provided. This model was implemented in 
a computer program called EPIM (Elastic-Plastic Impact Model) and the results were compared to numerous experiments, first with traditional composites (Vlot, 1996) and later with FMLs (Vlot, 1998). In both comparisons the model estimated the stiffness of the panels with good agreement to the tests. The failure of the prepreg was estimated using the Tsai-Wu stress criterion. The main deficiencies identified by Vlot were that the model does not incorporate a failure criterion to determine the first failure energy level. Also, it cannot be used to estimate the permanent dent depth after impact or delamination. Another analytical approach for modelling the impact behaviour in ARALL has been developed by Sun et al. 1993 and $\mathrm{Wu}$ and Sun, 1993. A contact law for impact loading to estimate the force response of the impacted panels was proposed. They then used a series of static indentation tests to develop best-fit parameters for the relations. The contact force, $F$, is:

$$
F=k \alpha^{n}
$$

where $k$ is the contact rigidity, $\alpha$ is the indentation depth and $n$ is a power index. Next, the following method was used to account for the permanent deflection of metal panels subjected to impact.

$$
F=F_{m}\left(\frac{\alpha-\alpha_{o}}{\alpha_{m}-\alpha_{o}}\right)^{q}
$$

The above equation is used to model the unloading path of the impact event. The parameters $n$ and $q$ are typically determined from static indentation experiments. Equation 20 can be rearranged as shown in Equation 21.

$$
F=S\left(\alpha-\alpha_{o}\right)^{q}
$$


The unloading rigidity, $S$, is given in Equation 22.

$$
S=\frac{F_{m}}{\left(\alpha_{m}-\alpha_{o}\right)^{q}}
$$

It was also assumed that there is a critical indentation value, $\alpha_{p}$, below which there would be no permanent deformation as shown in Equation 23.

$$
\alpha_{o}= \begin{cases}S_{p}\left(\alpha_{m}-\alpha_{p}\right) & \alpha_{\mathrm{m}}>\alpha_{\mathrm{p}} \\ 0 & \alpha_{m} \leq \alpha_{p}\end{cases}
$$

The parameters $k, q, S_{p}$ and $\alpha_{p}$ were determined using a $19.05 \mathrm{~mm} \times 25.4 \mathrm{~mm}$ specimen loaded statically. These values are read directly from loading and unloading force deflection curves from static indentation tests. This method is very limited in its applicability and has no capability for predicting delamination or other internal damage in FMLs. It can only be applied to estimate the contact force in the impacted panel.

\subsubsection{Delamination Damage Modelling for FMLs}

Current FML delamination research is mainly focused on delamination damage associated with spliced and notched FML coupons. At present there appears to be no work published describing delamination models for application to impact in FMLs. Delamination in FMLs can be influenced by geometry and material discontinuities, and consists of mode I, II and III fracture behaviour (Figure 48). 


\section{Mode I}

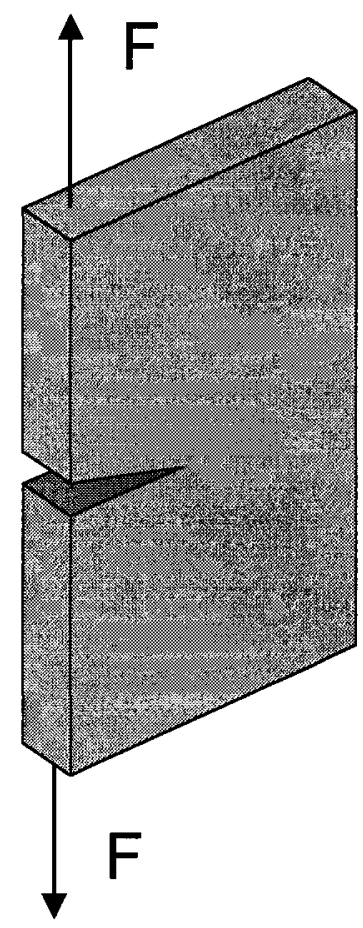

Mode II

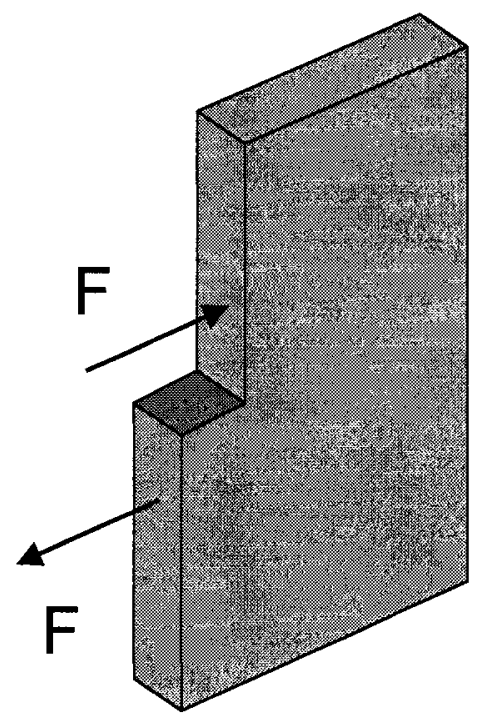

Mode III

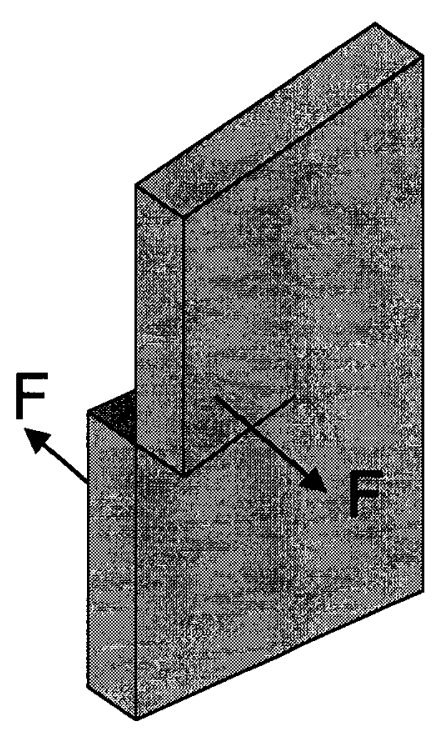

Figure 48: Schematic of fracture modes.

The main focus of this research has been delamination growth associated with the formation of fatigue cracks in the aluminum layers of FML sheets. This type of delamination is governed primarily by the mode II fracture toughness of the FML material. Table 12 summarizes current research. Initiation of delamination in GLARE was not covered in any of these methods. Lin and Kao (1996) and Guo et al. (1997) present in-depth approaches for predicting delamination growth. Other researchers have examined aspects of delamination behaviour in FMLs and their work will also be discussed in this review. 
Table 12: Summary of FML delamination research to date.

\begin{tabular}{|c|c|c|c|c|c|}
\hline Reference & Material & $\begin{array}{l}\text { Delamination } \\
\text { Type } \\
\end{array}$ & $\begin{array}{c}\text { Specimen } \\
\text { Type }\end{array}$ & $\begin{array}{l}\text { Numerical } \\
\text { Model Type }\end{array}$ & Comments \\
\hline $\begin{array}{l}\text { Hashagen } \\
\text { et al. } \\
(1999)\end{array}$ & GLARE-2 & $\begin{array}{l}\text { Delamination } \\
\text { growth in a } \\
\text { splice }\end{array}$ & Numerical only & $\begin{array}{l}\text { FE model with } \\
\text { interface } \\
\text { elements }\end{array}$ & Growth only - mode II \\
\hline $\begin{array}{l}\text { Vlot et al. } \\
\text { (1998) }\end{array}$ & $\begin{array}{l}\text { GLARE } \\
\text { and } \\
\text { ARALL }\end{array}$ & $\begin{array}{l}\text { Delamination } \\
\text { growth in } \\
\text { bonded FML } \\
\text { doublers }\end{array}$ & $\begin{array}{l}\text { Doubler } \\
\text { specimen } \\
\text { (cyclic) } \\
\text { DCB and ENF }\end{array}$ & None used & $\begin{array}{l}\text { Effect of post- } \\
\text { stretching on } \\
\text { delamination and } \\
\text { mixed mode }\end{array}$ \\
\hline $\begin{array}{l}\text { Guo et al. } \\
(1997)\end{array}$ & GLARE-2 & $\begin{array}{l}\text { Characterization } \\
\text { of delamination } \\
\text { growth }\end{array}$ & DCLS & $\begin{array}{l}\text { Strain energy } \\
\text { release rate }\end{array}$ & Growth only - Mode II \\
\hline $\begin{array}{l}\text { Lin and } \\
\text { Kao } \\
(1996)\end{array}$ & CARALL & $\begin{array}{l}\text { Characterization } \\
\text { of delamination } \\
\text { growth }\end{array}$ & SEN (cyclic) & $\begin{array}{l}\text { Strain energy } \\
\text { release rate }\end{array}$ & Growth only - mode II \\
\hline $\begin{array}{l}\text { Yeh et al. } \\
\text { (1988) }\end{array}$ & ARALL & $\begin{array}{l}\text { Fracture } \\
\text { mechanics of } \\
\text { delamination }\end{array}$ & Numerical only & $\begin{array}{l}\text { Stress } \\
\text { singularity at } \\
\text { delamination } \\
\text { tip }\end{array}$ & $\begin{array}{l}\text { Numerical only, } \\
\text { examined energy } \\
\text { release rates - Mode } \\
\text { I and Mode II } \\
\text { separately }\end{array}$ \\
\hline
\end{tabular}

Lin and Kao (1996) examined the effects of delamination on the propagation of fatigue cracks in CARALL specimens. They used a strain energy release approach to model the growth of the delamination front around the cracked region with a single-edge notched (SEN) specimen. This specimen is divided into several regions corresponding to the physical state of the panel under load. To predict the delamination they developed an expression for the effective stress intensity range $\left(\Delta K_{\text {eff }}\right)$ for the CARALL material. This model was used to predict the crack growth behaviour of CARALL sheets with an initial crack $\left(a_{0}\right)$ of $6 \mathrm{~mm}$. Experiments were employed to determine the delamination size corresponding to this initial flaw size. With a known crack growth rate for $a_{o}$, the corresponding effective stress intensity range can be found from the Paris Equation. The results of the analytical simulations compared well to experiments using SEN specimens in several different laminate configurations. This research demonstrated that the strain energy release approach for predicting delamination growth can be applied to FMLs. 
A slightly different approach was employed by Guo et al. (1997) for predicting the delamination growth behaviour in a double crack lap shear (DCLS) specimen of GLARE-2-2/1. The specimen was manufactured with an electric-discharge machined slit in each of the two aluminum layers. Cracks propagate through the aluminum layers to the fibre core. The damage then grows in the form of a delamination front along the interface between the aluminum and fibre layers. Guo et al. found that the compliance of the DCLS specimen increased linearly with the delamination size. They also observed that the growth of the delamination was predominately controlled by mode II behaviour, with some mode I influence. The energy release rate method was chosen to predict the delamination growth behaviour. Once this expression for the energy release rate was developed, a series of fatigue tests with the DCLS specimen for several stress ratios $(\mathrm{R}=0.1,0.3$ and 0.5$)$ was performed. The tests showed good agreement between the experimental data and the energy release rate approximations.

In 1988, Yeh et al. examined the fracture mechanics of delamination in ARALL laminates. Singular finite elements were used to model the delamination crack tip. This allowed the determination of the stress intensity factors and energy release rates for mode I and mode II delamination. It was found that mode II was the dominant form of delamination growth for the DCLS configuration. The stress intensity factors and energy release rates were independent of the size of the delamination. The predictions were compared to the experimental results of Awerbuch (1987) with good agreement.

Hashagen et al. (1999) carried out numerical investigations of the delamination behaviour of spliced FMLs based on the characterization experiments carried out by Vries et al. (1999) as described in Section 3.1.1. 


\subsubsection{Delamination Modelling for Composites}

There are also numerous models and approaches in literature for the prediction of delamination initiation in fibre-reinforced composites. Several of these models are reviewed below. These methods are different from the ones described previously in that they predict the initiation of delamination damage, not its growth.

Brewer and Lagace (1988) developed the Quadratic Delamination Criterion (Equation 24) for estimating the initiation of delamination damage in laminated composites. This model uses an average stress criterion to compare the calculated out-ofplane interlaminar stresses to the appropriate material strength parameters.

$$
\left(\frac{\bar{\sigma}_{13}}{s_{13}}\right)^{2}+\left(\frac{\bar{\sigma}_{23}}{s_{23}}\right)^{2}+\left(\frac{\bar{\sigma}_{33}^{t}}{s_{33}^{t}}\right)^{2}+\left(\frac{\bar{\sigma}_{33}^{c}}{s_{33}^{c}}\right)^{2}=1
$$

The superscripts $t$ and $c$ in the above equation denote tensile and compressive stresses.

Another strength of materials approach was developed by Choi and Chang (1992). Their criterion assumes that delamination initiates from matrix cracks within the layers. They predict the occurrence of a critical matrix crack with a criterion of the form:

$$
\left(\frac{\bar{\sigma}_{22}}{s_{22}}\right)_{n}^{2}+\left(\frac{\bar{\sigma}_{23}}{s_{23}}\right)_{n}^{2} \geq 1
$$

where the subscript $n$ refers to the $n^{\text {th }}$ ply layer in the specimen. When the critical matrix crack size in a given layer has been reached, Choi and Chang proposed the following delamination criterion:

$$
D_{a}\left[\left(\frac{\bar{\sigma}_{23}}{s_{23}}\right)_{m}^{2}+\left(\frac{\bar{\sigma}_{13}}{s_{13}}\right)_{m}^{2}+\left(\frac{\bar{\sigma}_{22}}{s_{22}}\right)_{m}^{2}\right] \geq 1
$$

where the value $D_{a}$ is an empirical constant determined through experiments. 
A method for predicting the failure of an interface based upon a strength-ofmaterials approach was described by Chang and Springer (1987). This formula, shown in Equation 27, compares the normal and shear stresses applied to the interface with the interlaminar normal and shear strength parameters $\left(s_{n}\right.$ and $\left.s_{s}\right)$. The strength parameters are obtained from either manufacturer's data or interface strength tests.

$\left(\frac{\left|\sigma_{n}\right|}{s_{n}}\right)^{2}+\left(\frac{\left|\sigma_{s}\right|}{s_{s}}\right)^{2} \geq d^{2}$

if $d<1$ then there is no failure

if $d \geq 1$ then there is complete failure

\subsection{Proposed Modelling Methodology}

The experimental testing described in Chapter 2 demonstrated the impact response of GLARE under specific conditions. However, these experiments were limited to only three types of GLARE, with only a single boundary condition. In service these materials could be subjected to many types of impact events. As well, the lay-up and boundary conditions could vary from location to location on an aircraft. The number of experiments required to adequately characterize the response of these materials would be large. Accurate impact damage simulations could reduce this testing requirement.

The review presented in the previous section showed that there is, as yet, no practical methodology for predicting impact induced damage in FMLs that incorporates all of the important damage modes. Such a methodology must be able to predict not only the measured response (absorbed energy, force and dent depth) but also the geometry of the damage developed in the panel. However, some aspects of the previously developed 
models can be applied to the problem of predicting impact damage in FMLs. For example, finite element methods offer advantages over analytical techniques. Finite element methods can be used to model many types of geometries and boundary conditions by changing the input parameters. As well, finite element models can be scaled up to simulate entire components and structures. Multi-layered models can be constructed using finite elements with a damageable interface between the layers. Some commercial finite element codes, such as LS-DYNA, also make it possible to implement user-defined material models directly as part of the code. The individual materials that compose the GLARE laminates can thus be characterized separately before incorporation in the finite element model. The analytical models presented previously, required characterization of each new laminate type. As well, analytical models are usually developed for a specific geometry and loading condition and this limits their usefulness. However, analytical models are generally computationally efficient for simple problems.

The proposed damage modelling methodology requires four components to predict the impact response of the FML panels as shown in Figure 49. The aluminum material model must be able to accurately predict the elastic and plastic response of the metal layers under impact loading. The next component is the damage model for the prepreg layers. This model is required to capture the effect of matrix cracking on the impact response. The final two components, the delamination initiation and postdelamination models, are closely related. Without an accurate initiation model, delamination damage may be predicted too early, too late or not at all in the impact simulations. The post-delamination behaviour model is required to predict the effects of the fibre-bridging that was observed in the experiments described in Section 2.2.4. 


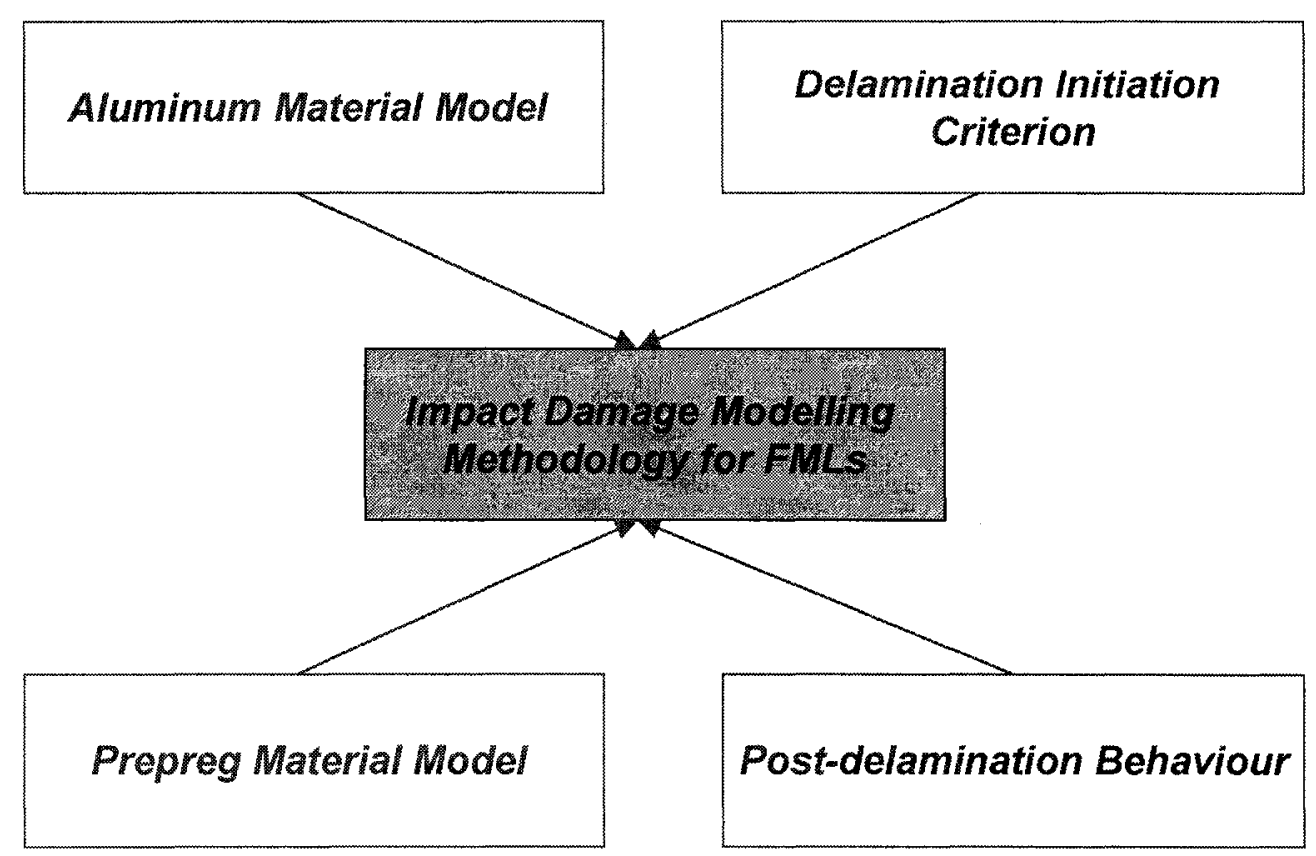

Figure 49: Required components of the FML damage modelling methodology.

Matrix plasticity and strain-rate sensitivity were shown to have a minor effect on the response of FMLs subjected to low-velocity impact (Vlot, 1993b). These effects will not be incorporated at this time. Also, the current objective is to model FMLs below the puncture threshold. Since fibre fracture was only observed in punctured specimens, a prediction for this damage mode will not be employed as part of this methodology.

Following the development and validation of the individual components, the damage modelling methodology was used to model low-velocity impacts on GLARE as described in Chapter 7. These simulations used energy levels identical to those employed for the experiments described in Chapter 2. Figure 50 shows schematically the relationship between the various tasks completed as part of the development of this methodology. As indicated in the flowchart, some of the required components already exist in the explicit finite element code LS-DYNA. 


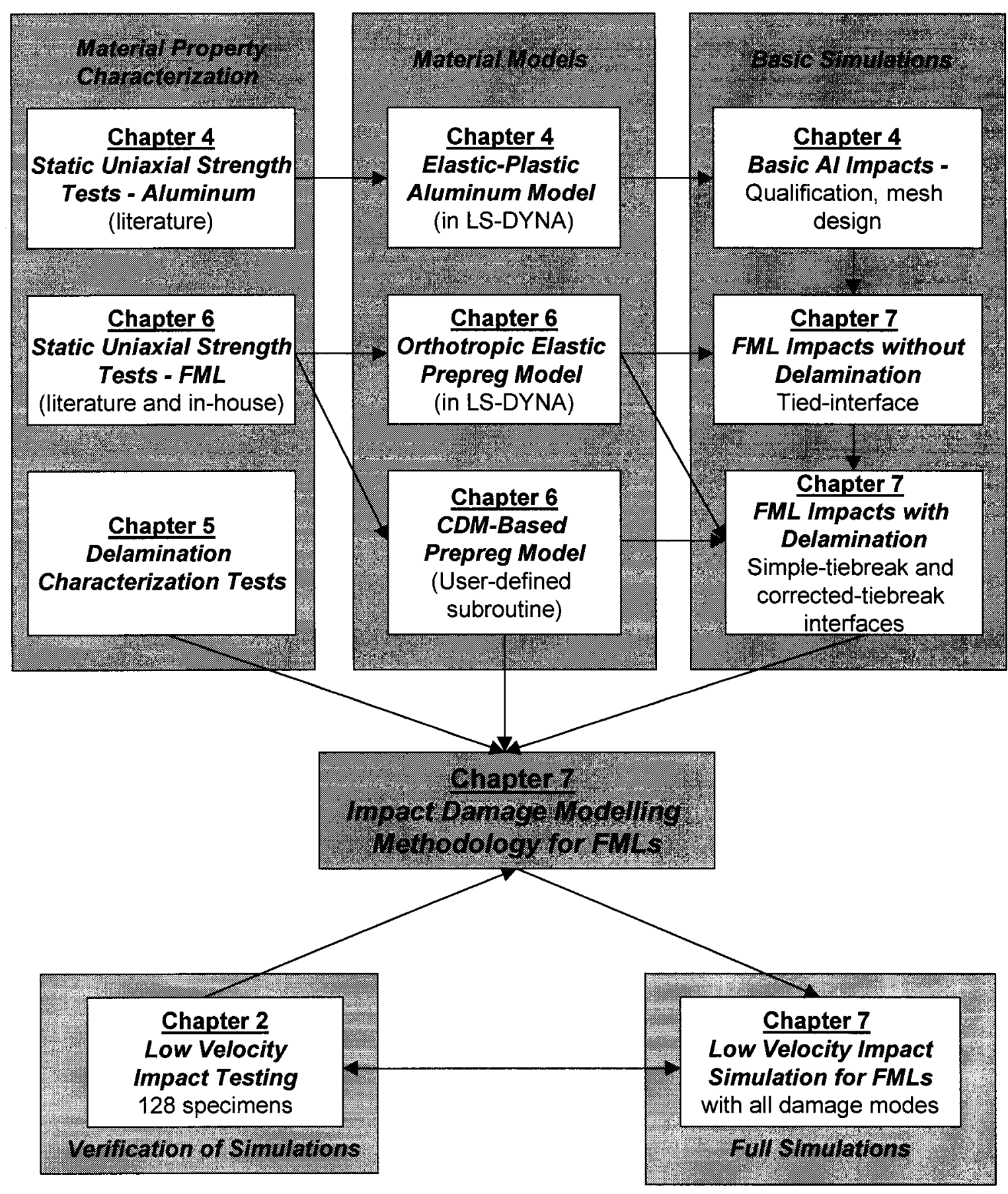

Figure 50: Modelling methodology components and task relationships. 


\subsection{Overview of the LS-DYNA Explicit}

\section{Finite Element Code}

To develop a practical methodology for modelling the damage formed in FMLs, a commercial finite element code was used instead of developing a stand-alone, single purpose program. One commercial code that is well suited for modelling impact and non-linear problems is LS-DYNA. This code is a general purpose, vectorized, explicit finite element program. It has the capability to model $2 \mathrm{D}$ and $3 \mathrm{D}$ problems with large deformations. LS-DYNA was developed at the Livermore National Laboratory (Hallquist, 1998) for explosives and crashworthiness simulations. Its applicability has expanded to include low-velocity impact and metal forming among other applications. This code has been successfully applied to model the behaviour of composites under impact by other researchers (van Hoof, 1999, Hung, 1995, Majeed, 1994, Williams and Vaziri, 1995 and Williams and Vaziri, 2001).

The central difference method is used to solve the equations of motion with respect to time. This explicit finite element method uses time steps rather than load steps. Each time step must be smaller than a critical value to ensure the stability of the solution. The critical time step is a function of a key dimension of the element such as the length of the shortest side, and the speed of sound in the material. The sound wave should not cross the element in one time step. The smallest time step calculated from all of the elements is used as the critical time step for the integration. This time step is recalculated with each subsequent solution of equations. 
There are three types of elements that were used in this thesis and each has distinct properties. The four-node thin-shell (Belytschko-Tsay) elements require the least amount of CPU time and are useful for simulating thin panels. The eight-node thickshells are actually a hybrid of thin-shells and solid-brick elements. However, because they are 3D they are particularly useful in modelling multi-layered thin panels with discrete interlaminar interfaces. Eight-node solid-brick elements are also useful in modelling multi-layered panels but require larger amounts of CPU time. They are not suited for thin panels unless a large number of elements is used to keep the aspect ratio below 10:1. Each element type is discussed in more detail below.

Another important feature of LS-DYNA is its use of contact algorithms to model the interfaces between parts. These can be tied-, tiebreak- or sliding-interfaces. Static and kinetic coefficients of friction can also be defined for the interface. All of these types of interface are used in this thesis. Version 950e of LS-DYNA was employed in this project and, unlike older versions, it makes use of keywords to activate the different features. Table 13 gives the keywords and presents how they are referred to in this thesis. Each of these features is discussed in greater detail in the sections to follow.

Table 13: Important features of LS-DYNA used in the simulation of GLARE and aluminum subjected to low-velocity impact.

\begin{tabular}{|l|c|}
\hline \multicolumn{1}{|c|}{ Keyword } & Referred to as: \\
\hline${ }^{*}$ MAT_ELASTIC & elastic (type 1) \\
\hline${ }^{*}$ MAT_ORTHOTROPIC_ELASTIC & orthotropic-elastic (type 2) \\
\hline${ }^{*}$ MAT_RIGID & rigid (type 20) \\
\hline${ }^{*}$ MAT_PIECEWISE_LINEAR_PLASTICITY & elastic-plastic (type 24) \\
\hline${ }^{*}$ MAT_USER_DEFINED_MATERIAL_MODELS & UMAT \\
\hline *CONTACT_TIED_SURFACE_TO_SURFACE & tied-interface \\
\hline${ }^{*}$ CONTACT_TIEBREAK_SURFACE_TO_SURFACE & tiebreak-interface \\
\hline${ }^{*}$ CONTACT_AUTOMATIC_SURFACE_TO_SURFACE & \\
\hline${ }^{*}$ ELEMENT_TSHELL & thick-shell \\
\hline "ELEMENT_SHELL & thin-shell \\
\hline *ELEMENT_SOLID & solid-brick \\
\hline
\end{tabular}


As with any finite element code, it is important to use consistent units for all the input parameters. The basic units that are selected by the user should be on the same order of magnitude as the basic dimensions of the structure under analysis. Table 14 lists the units for each of the major parameters used in this thesis.

Table 14: Units used in LS-DYNA simulations.

\begin{tabular}{|l|l|}
\hline Length & millimeter $(\mathrm{mm})$ \\
\hline Time & millisecond $(\mathrm{ms})$ \\
\hline Mass & kilogram $(\mathrm{kg})$ \\
\hline Force & kiloNewton $(\mathrm{kN})$ \\
\hline Stress & $\mathrm{kN} / \mathrm{mm}^{2}=\mathrm{GPa}$ \\
\hline Velocity & $\mathrm{mm} / \mathrm{ms}=\mathrm{m} / \mathrm{s}$ \\
\hline Acceleration & $\mathrm{mm} / \mathrm{ms}^{2}$ \\
\hline Energy & $\left(\left(\mathrm{kg}{ }^{\star} \mathrm{mm}\right) / \mathrm{ms}^{2}\right)^{\star} \mathrm{mm}=\mathrm{J}$ \\
\hline Density & $\mathrm{kg} / \mathrm{mm}^{3}$ \\
\hline
\end{tabular}

\subsubsection{Thin-Shell Elements}

The Belytschko-Lin-Tsay (Belytschko et al. 1984) element is the default thinshell formulation implemented in LS-DYNA. This element, which is based upon a combined co-rotational and velocity-strain formulation, was developed to optimize computational efficiency. An embedded element coordinate system is defined in terms of the reference surface of the element (Hallquist, 1998). This coordinate system deforms with the shell element. With this formulation the element strain is restricted to small values but the out-of-plane deformations are not. The velocity-strain displacement relations are also restricted to small strains. The Belytschko-Lin-Tsay element follows the Mindlin theory of plates and shells to partition the displacement of any point in the shell to a mid-surface displacement (nodal translation) and a displacement associated with the rotation of the element's fibres (nodal rotations). 
In LS-DYNA, contact interfaces are created by defining master and slave surfaces. Thin-shell elements only have one layer of nodes; therefore, nodes must be defined as being part of a master surface and a slave surface simultaneously. This can cause numerical problems that can be avoided if thick-shell elements or solid-brick elements are used.

As in most finite element codes, thin-shells in LS-DYNA can be used to represent composite plies by defining multiple integration points in the element. In most codes, the orientation and material properties of each ply can be assigned to model multi-directional laminates. In LS-DYNA one can only assign different ply angles to each integration point. It is not possible to assign different material properties to each point. This reinforces the unsuitability of the thin-shells in LS-DYNA for modelling FMLs.

\subsubsection{Thick-Shell Elements}

The thick-shell element is recommended for applications where thick-walled shell structures are to be modelled. Also, this element permits the modelling of multi-layered structures so that failure of the interface between different layers can be predicted. These elements also minimize potential problems with defining the contact interfaces that can arise when using thin-shell elements. Thick-shell elements are geometrically identical to traditional 8-node solid-bricks with four nodes on the top and bottom surfaces. Each group of four nodes can be assigned to a separate contact segment for the top and bottom surfaces of the layer. This avoids the problem of assigning one node to two different contact segments simultaneously. 
The geometry of the element is interpolated in the same manner as the eight-node solid element (Hallquist, 1998):

$$
x_{i}\left(X_{\alpha}, t\right)=x_{i}\left(X_{\alpha}(\xi, \eta, \zeta), t\right)=\sum_{j=1}^{8} \phi_{j}(\xi, \eta, \zeta) x_{i}^{j}(t)
$$

The shape functions for this element are defined as:

$$
\phi_{j}=\frac{1}{8}\left(1+\xi \xi_{j}\right)\left(1+\eta \eta_{j}\right)\left(1+\zeta \zeta_{j}\right)
$$

A schematic of this element is shown in Figure 51. The co-ordinates of the nodes take on the values of $( \pm 1, \pm 1, \pm 1)$ with $x_{i}^{j}$ as the nodal co-ordinate of the $j$ th node in the ith direction. The interpolation matrix, $N$, for the solid-like shell element is similar to that for solid elements:

$$
N(\xi, \eta, \zeta)=\left[\begin{array}{cccccccc}
\phi_{1} & 0 & 0 & \phi_{2} & 0 & \ldots & 0 & 0 \\
0 & \phi_{1} & 0 & 0 & \phi_{2} & \ldots & \phi_{8} & 0 \\
0 & 0 & \phi_{1} & 0 & 0 & \ldots & 0 & \phi_{8}
\end{array}\right]
$$

The stress vector, $\sigma$, for the element is given by:

$$
\sigma^{t}=\left(\sigma_{x x}, \sigma_{y y}, \sigma_{z z}, \sigma_{x y}, \sigma_{y z}, \sigma_{z x}\right)
$$

Finally, the $6 \times 24$ strain-displacement matrix, $B$, is expressed as: 


$$
B=\left[\begin{array}{ccc}
\frac{\partial}{\partial x} & 0 & 0 \\
0 & \frac{\partial}{\partial y} & 0 \\
0 & 0 & \frac{\partial}{\partial z} \\
\frac{\partial}{\partial y} & \frac{\partial}{\partial x} & 0 \\
0 & \frac{\partial}{\partial z} & \frac{\partial}{\partial y} \\
\frac{\partial}{\partial z} & 0 & \frac{\partial}{\partial x}
\end{array}\right] N
$$

Next the terms within the strain-displacement matrix are found as:

$$
\left[\begin{array}{l}
\frac{\partial \phi_{i}}{\partial \xi} \\
\frac{\partial \phi_{i}}{\partial \eta} \\
\frac{\partial \phi_{i}}{\partial \zeta}
\end{array}\right]=\left[\begin{array}{lll}
\frac{\partial x}{\partial \xi} & \frac{\partial y}{\partial \xi} & \frac{\partial z}{\partial \xi} \\
\frac{\partial x}{\partial \eta} & \frac{\partial y}{\partial \eta} & \frac{\partial z}{\partial \eta} \\
\frac{\partial x}{\partial \zeta} & \frac{\partial y}{\partial \zeta} & \frac{\partial z}{\partial \zeta}
\end{array}\right]\left[\begin{array}{c}
\frac{\partial \phi_{i}}{\partial x} \\
\frac{\partial \phi_{i}}{\partial y} \\
\frac{\partial \phi_{i}}{\partial z}
\end{array}\right]=J\left[\begin{array}{c}
\frac{\partial \phi_{i}}{\partial x} \\
\frac{\partial \phi_{i}}{\partial y} \\
\frac{\partial \phi_{i}}{\partial z}
\end{array}\right]
$$

As with typical finite element methods we must then invert the Jacobian matrix, $J$, and solve for the desired terms to give:

$$
\left[\begin{array}{l}
\frac{\partial \phi_{i}}{\partial x} \\
\frac{\partial \phi_{i}}{\partial y} \\
\frac{\partial \phi_{i}}{\partial z}
\end{array}\right]=J^{-1}\left[\begin{array}{c}
\frac{\partial \phi_{i}}{\partial \xi} \\
\frac{\partial \phi_{i}}{\partial \eta} \\
\frac{\partial \phi_{i}}{\partial \zeta}
\end{array}\right]
$$




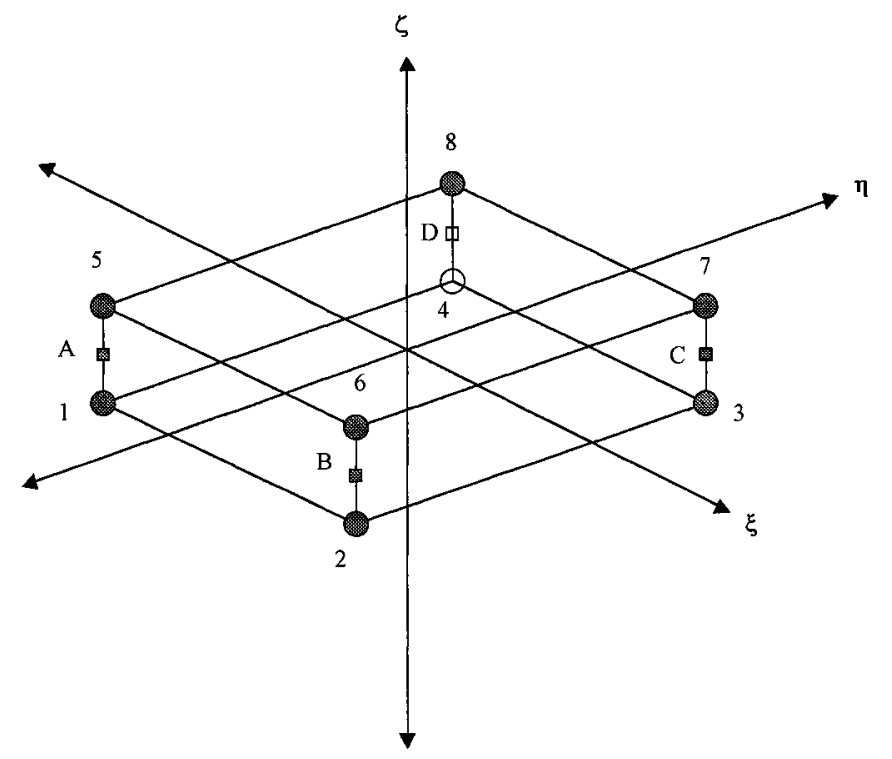

Figure 51: Nodal locations for the LS-DYNA thick-shell element.

A reference surface is located midway between the upper and lower surfaces of the element and is constructed in a manner identical to the Belytschko-Lin-Tsay thin-shell element in LS-DYNA (Belytschko et al. 1984). A local co-ordinate system is constructed for the reference surface that deforms with the element. Similar to other elements that use single-point integration in LS-DYNA, thick-shell elements require the use of hourglass control. This will be discussed in greater detail in Section 3.3.4.

\subsubsection{Solid-Brick Elements}

The basic formulation of the standard LS-DYNA solid-brick element is similar to that of the thick-shell element as described in Equations (28-34). The main difference is that this element does not use a reference surface. The element uses a single-point integration scheme that significantly reduces computation time over fully-integrated elements (Hallquist, 1998). However, this reduced integration scheme creates the requirement for hourglass control. 


\subsubsection{Hourglass Control}

Hourglass control is necessary whenever element formulations are employed that are not fully integrated, as is the case with the thick-shells and solid-bricks in LS-DYNA. Hourglass modes are zero-energy deformation modes that have a period much shorter than the structural response period. These modes are orthogonal to the strain calculations in the element and as such there are no internal forces to resist the deformations (Flanagan and Belytschko, 1981). They arise due to the use of single point integration in the finite elements.

There are two methods for resisting the formation of these modes in LS-DYNA. The first, referred to as the "stiffness method", employs a small elastic stiffness that resists the anomalous modes. The stiffness method has been identified as being suitable for low-velocity simulations. This method slows, stops and undoes the hourglass deformation modes. In large deformation problems, this method may over-stiffen the model. The second method, known as the "viscosity method", is the default hourglass control method in LS-DYNA. With this method, the hourglass deformations are slowed and stopped but not undone. However, the viscosity method is recommended in highvelocity problems with large deformation and was used in this thesis.

Despite the problems caused by hourglassing modes, single point integration can result in substantial computational savings. For example, compared with full integration, single-point integration reduces computational time by a factor of 16 for strain and nodal force computations and by a factor of 8 for stresses (Hallquist, 1998). The computational cost of implementing hourglass control measures is minor compared to the cost of using fully-integrated elements in explicit finite element analysis (Hallquist, 1998). 


\subsubsection{Interface Models}

The LS-DYNA code incorporates four distinct methods of modelling the contact between two surfaces. Hallquist (1998) described these algorithms. The first method of contact definition is referred to as the "kinematic constraint method". This algorithm is currently only employed in tied-interfaces, as in the interface between the prepreg and the aluminum layers of GLARE. In this method the displacements of the slave nodes are tied to the master surface through a global transformation of the nodal displacements. This effectively eliminates the normal component of displacement for these nodes. Momentum is conserved by imposing impact and release constraints as described by Hughes et al. (1976). The kinematic constraint method is used for both the tied- and tiebreak-interfaces in LS-DYNA.

The second method of contact modelling is the "penalty method". The interpenetration of the surfaces in contact is resisted by inserting springs between the surfaces. The springs resist penetration and force the surfaces apart. This method of contact simulation is used in surface-to-surface contact with friction, as takes place between the impactor and the panels.

The remaining two contact algorithms are the "surface to surface constraint method" and the "distributed parameter method". Both are used in very limited applications. For example, the distributed parameter method is used to model only sliding in fluid-structure or gas-structure interaction problems.

These contact algorithms are used to model the interfaces between parts in several ways. Groups of elements can be connected using a tied-interface that constrains rotations and displacements of the slave nodes with the master surface. It is also possible 
to model a sliding interface between two surfaces with or without friction. This type of interface can be used to model the contact between the impactor and the panels.

It is possible to model the failure and propagation of interlaminar failures in LSDYNA using tiebreak contact based on the kinematic constraint method. The failure of the interface is predicted using the relationship developed by Chang and Springer (1986) as presented in Equation 27 of Section 3.1.4. Once the nodes are released, contact between the interfaces is accounted for using the penalty method. Tiebreak contact has been used to successfully model the failure of interfaces between layers of composites (van Hoof, 1999 and Hung, 1995). Post-failure separation of the two surfaces can be modelled with a user-defined piecewise-linear stress-displacement curve. This could be used to simulate the effect of fibre bridging on the delamination growth. The basic, uncorrected interface is referred to as the simple-tiebreak interface in this thesis. The term corrected-tiebreak interface indicates the use of the stress-displacement curve. 


\subsubsection{Material Models}

The elastic-plastic behaviour of metals is simulated in LS-DYNA using the elastic-plastic (type 24) material model in LS-DYNA. The stress-strain curve for the material is divided into discrete linear portions and entered into the code. The prepreg layers can be represented using the standard LS-DYNA orthotropic-elastic (type 2) material. This model is appropriate only for elastic deformations and does not take into account plasticity or damage effects. The constitutive matrix in terms of the material axes is (Hallquist, 1998):

$$
C_{l}^{-1}=\left[\begin{array}{cccccc}
\frac{1}{E_{11}} & -\frac{v_{21}}{E_{22}} & -\frac{v_{31}}{E_{33}} & 0 & 0 & 0 \\
-\frac{v_{12}}{E_{11}} & \frac{1}{E_{22}} & -\frac{v_{32}}{E_{33}} & 0 & 0 & 0 \\
-\frac{v_{13}}{E_{11}} & -\frac{\nu_{23}}{E_{22}} & \frac{1}{E_{33}} & 0 & 0 & 0 \\
0 & 0 & 0 & \frac{1}{G_{12}} & 0 & 0 \\
0 & 0 & 0 & 0 & \frac{1}{G_{23}} & 0 \\
0 & 0 & 0 & 0 & 0 & \frac{1}{G_{31}}
\end{array}\right]
$$

The stress and strain matrices are related to each other as:

$$
S=C E=T^{t} C_{l} T E
$$

where $T$ is the transformation matrix to transform the global co-ordinates into the local material system and $T^{t}$ is the transpose of this matrix. For the plane-stress condition the relationship for the incremental stress update is:

$$
\sigma_{l}^{n+1}=\sigma_{l}^{n}+\Delta \sigma_{l}^{n+1 / 2}
$$

with 


$$
\Delta \sigma_{l}^{n+1 / 2}=\left[\begin{array}{c}
\sigma_{11} \\
\sigma_{22} \\
\sigma_{12} \\
\sigma_{23} \\
\sigma_{31}
\end{array}\right]=\left[\begin{array}{ccccc}
\frac{E_{11}}{1-v_{12} v_{21}} & \frac{v_{12} E_{22}}{1-v_{12} v_{21}} & 0 & 0 & 0 \\
\frac{v_{12} E_{22}}{1-v_{12} v_{21}} & \frac{E_{22}}{1-v_{12} v_{21}} & 0 & 0 & 0 \\
0 & 0 & G_{12} & 0 & 0 \\
0 & 0 & 0 & G_{23} & 0 \\
0 & 0 & 0 & 0 & G_{31}
\end{array}\right]\left[\begin{array}{c}
\dot{\varepsilon}_{11} \\
\dot{\varepsilon}_{22} \\
\dot{\varepsilon}_{12} \\
\dot{\varepsilon}_{23} \\
\dot{\varepsilon}_{31}
\end{array}\right]_{l}
$$

The out-of-plane strain, $\varepsilon_{33}$, needs to be calculated as part of the material model.

For the elastic case this strain is simply:

$$
\varepsilon_{3}=\frac{C_{12}}{C_{33}} \varepsilon_{1}-\frac{C_{23}}{C_{33}} \varepsilon_{2}
$$

with $C_{13}, C_{23}$ and $C_{33}$ as follows:

$$
\begin{aligned}
& C_{13}=\frac{E_{1}\left(v_{32}+v_{21} v_{23}\right)}{1-v_{12} v_{21}-v_{23} v_{32}-v_{13} v_{31}-2 v_{12} v_{23} v_{31}} \\
& C_{23}=\frac{E_{2}\left(v_{32}+v_{12} v_{21}\right)}{1-v_{12} v_{21}-v_{23} v_{32}-v_{13} v_{31}-2 v_{12} v_{23} v_{31}} \\
& C_{33}=\frac{E_{1}\left(1-v_{12} v_{21}\right)}{1-v_{12} v_{21}-v_{23} v_{32}-v_{13} v_{31}-2 v_{12} v_{23} v_{31}}
\end{aligned}
$$

In addition to the standard material models that are part of LS-DYNA, it is also possible to incorporate user-defined subroutines (UMATs) directly into the program. The UMAT is written in FORTRAN and can be activated by the user with a keyword command in the input deck. Element stress and strain data are passed to the subroutine from the main program. The UMAT is then employed to update these values. The software also has the capability to store history variables that can be used to record damage levels or simply as step counters. 


\subsubsection{Application of LS-DYNA to Model Impact Damage in FMLs}

To summarize, LS-DYNA was selected to model the impact response of FML panels. First, simulations of monolithic aluminum panels subjected to low-velocity impact were conducted to compare the performance using thin-shell, thick-shell and solid-brick elements. Additionally, mesh sensitivity, boundary conditions, geometry effects and the sensitivity of the model to the material input parameters were examined.

Using the selected element type and the basic mesh geometry, a series of lowvelocity impact simulations of GLARE was conducted. The existing elastic-plastic (type 24) model was used for the aluminum layers. As observed through the destructive inspections, damage develops within the prepreg layers following impact. None of the standard material models in LS-DYNA could be applied to thick-shell elements to predict the effect of the development of damage on the impact response of the panels. Therefore, a user-defined material subroutine (UMAT) was developed to model the prepreg material. The user-defined material model was compared with the orthotropic-elastic (type 2) material model in LS-DYNA.

The interface between the aluminum and prepreg layers was modelled using the tied- and simple-tiebreak and corrected-tiebreak interfaces. The application of a postfailure correction to model the effects of fibre-bridging was also examined. All of the simulation results were compared to the experimental impact results from Chapter 2 . 


\section{CHAPTER 4}

\section{SIMULATIONS OF LOW-}

\section{VELOCITY IMPACT ON}

\section{ALUMINUM}

The elastic-plastic material (type 24) model in LS-DYNA is a simple representation of the development of plastic strain in an isotropic material. In order to qualify LS-DYNA to model low-velocity impact and to determine the basic input parameters, a series of simulations were conducted with 2024-T3 aluminum. In this Chapter, overviews of the material properties and the effect of mesh density variations are presented first. Next, a description of the preliminary results is provided with a comparison to the experimental impact data from Chapter 2. Lastly, a series of aluminum simulations with the final mesh geometry using thin-shells, thick-shells and solid-brick elements is presented. 


\subsection{Material Properties}

The material properties for the aluminum layers came from the sheet manufacturer, ALCOA. These properties are summarized in Table 15. The aluminum alloy layers were modelled using the manufacturer's stress-strain curve for 2024-T3 aluminum as shown in Figure 52.

Table 15: Summary of baseline material properties used for 2024-T3.

\begin{tabular}{|c|c|c|}
\hline Property & Value & Unit \\
\hline \hline Young's Modulus & 72.39 & $\mathrm{GPa}$ \\
\hline Yield Stress & 285 & $\mathrm{MPa}$ \\
\hline Mass Density & $2.643 \times 10^{-6}$ & $\mathrm{~kg} / \mathrm{mm}^{3}$ \\
\hline Poisson's Ratio & 0.33 & - \\
\hline
\end{tabular}

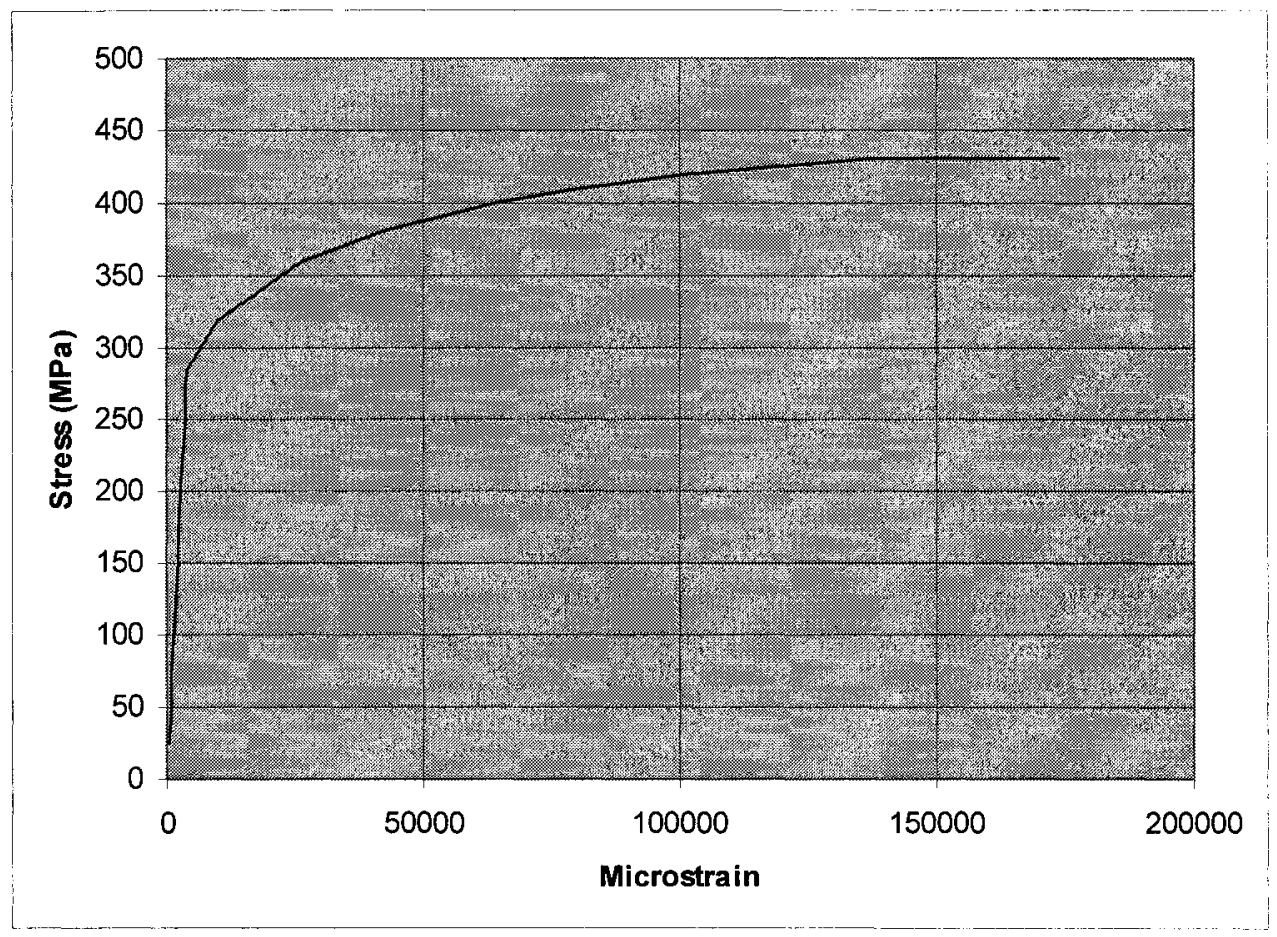

Figure 52: Manufacturer's stress-strain data for aluminum 2024-T3 sheet. 


\subsection{Mesh Density and Geometry}

The simulated geometry corresponded to the experimental set-up described in Section 2.2.3. Only the $203.2 \mathrm{~mm}$ diameter unsupported region of the impacted panel was modelled. The edges of the panel were either simply-supported to allow rotation or clamped to restrain all degrees of freedom. The aluminum panel thickness was 1.016 $\mathrm{mm}$, which is the same as the aluminum panels used in the experiments. The number of elements and nodes was varied to study the effect of mesh density on the simulation results. The geometry and meshes were prepared using IDEAS, a 3-D solid modelling package, and FEMB, a third-party FEA pre- and post-processor. The basic geometry of the finite element simulation is shown in Figure 53.

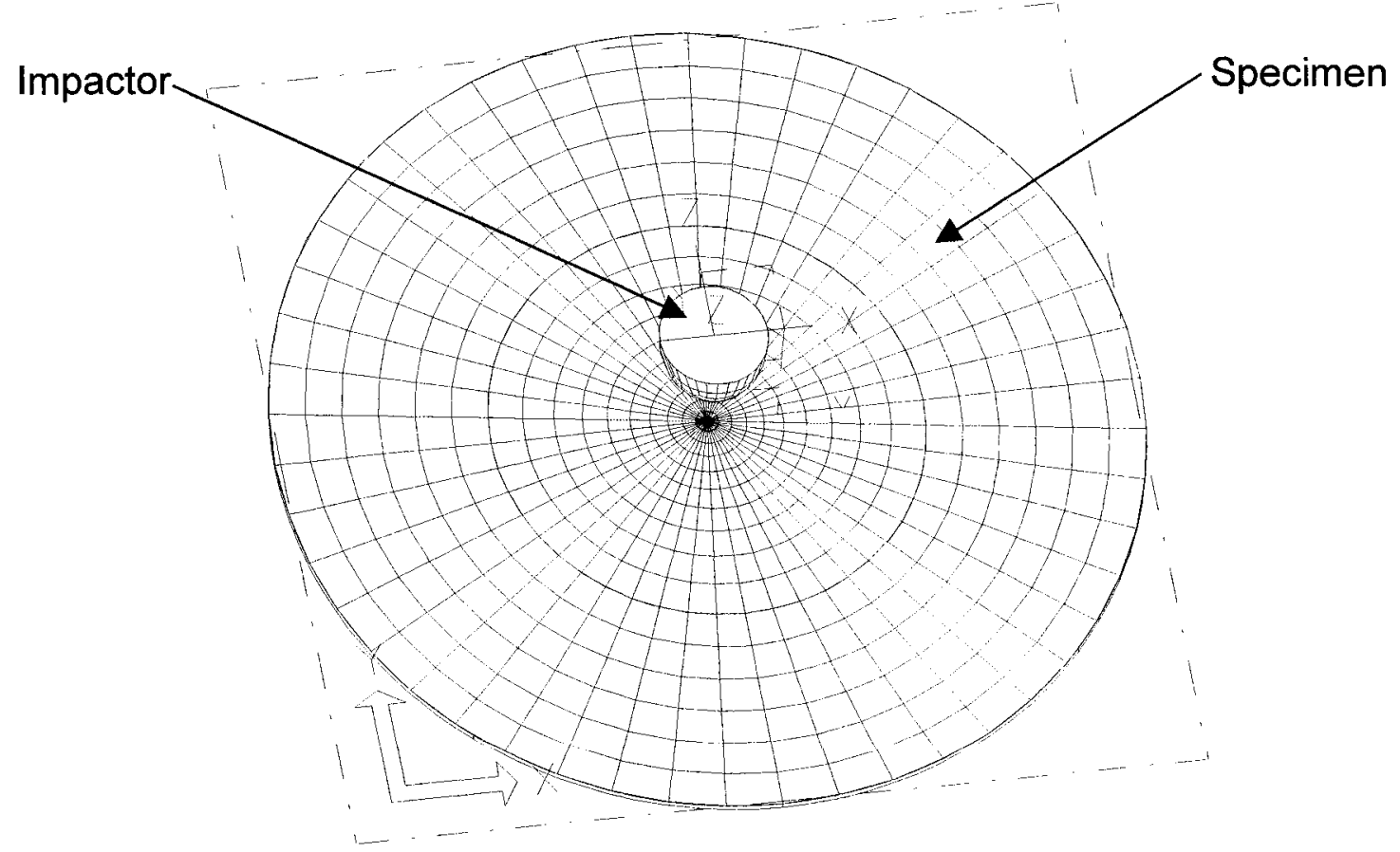

Figure 53: Basic impact geometry for the preliminary impact simulations. 
The $25.4 \mathrm{~mm}$ diameter hemispherical end of the impactor was modelled using 100 shell elements and rigid material (type 20). In LS-DYNA no stresses or deformations are calculated for rigid materials. However, some properties are still required for the determination of contact restoring forces. For this reason the modulus (200 GPa) and Poisson's ratio (0.33) of common steel were used. The mass of the drop weight hammer $(6.45 \mathrm{~kg})$ was incorporated into the model using discrete mass elements placed at each node of the hemispherical impactor. An initial velocity was applied to give the required kinetic energy for each impact event.

\subsection{Simulated Events}

A total of five energy levels were simulated with aluminum ( 25 to $65 \mathrm{~J})$. All simulations were performed on an SGI Indigo2 workstation with the UNIX operating system. There were also four different mesh geometries as follows:

1. "Rough" mesh - an unoptimized mesh, developed using IDEAS.

2. "Low" density mesh - developed with the element boundaries arranged radially and circumferentially. A bias was employed to increase the local density of the mesh at the point of impact.

3. "Medium" density mesh - developed with the element boundaries arranged radially and circumferentially. This mesh geometry was tested with simplysupported and clamped boundary conditions.

4. "High" density mesh - developed with the element boundaries arranged radially and circumferentially. 
The meshes are shown schematically in Figures 54 and 55. The "rough" edge-seeded mesh shown in Figure 54 was generated by specifying the number of elements along the edge of the panel. The mesh shown in Figure 55 possesses degenerated quadrilateral elements at the centre that my cause higher local stiffness.

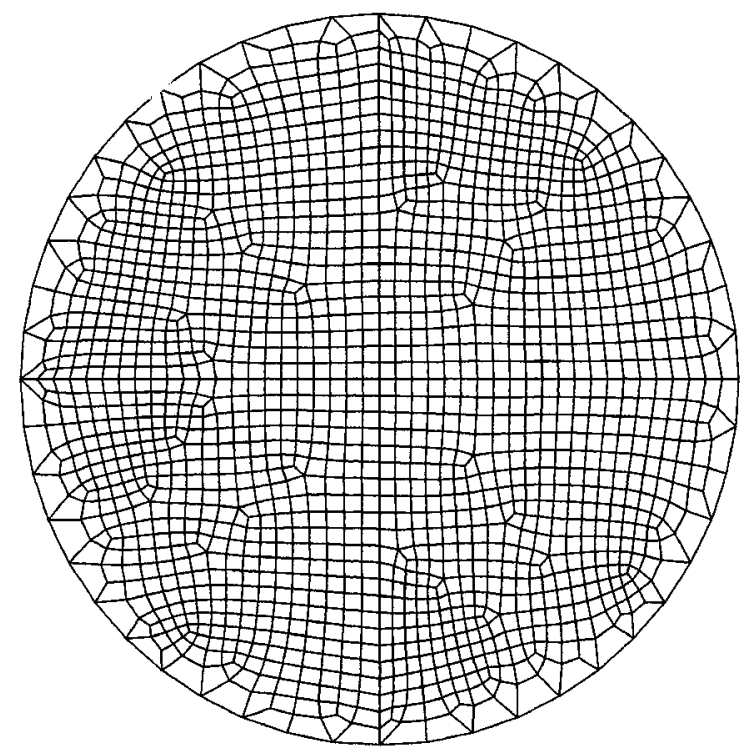

Figure 54: Edge-seeded "rough" mesh.

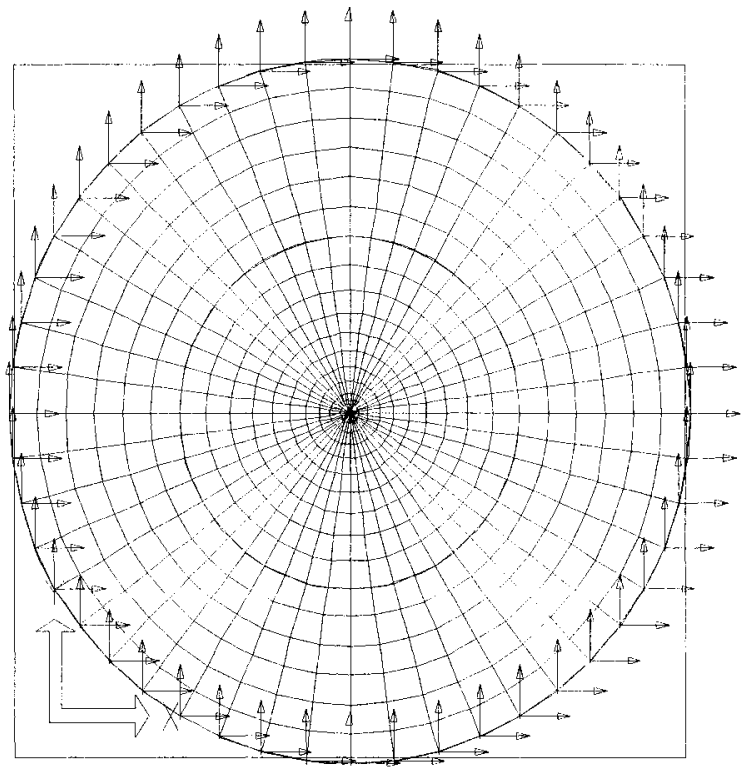

Figure 55: Basic mesh used for "low," "medium" and "high" mesh density simulations. 
The characteristics of the simulations are summarized in Table 16. The boundary conditions were changed for the "medium" density mesh to examine boundary condition effects on impact response. For each of the mesh densities a total of five energy levels were simulated. These corresponded to the different energy levels that were used in the experiments as described in Section 2.2.

Table 16: Simulation matrix for simple aluminum impact events.

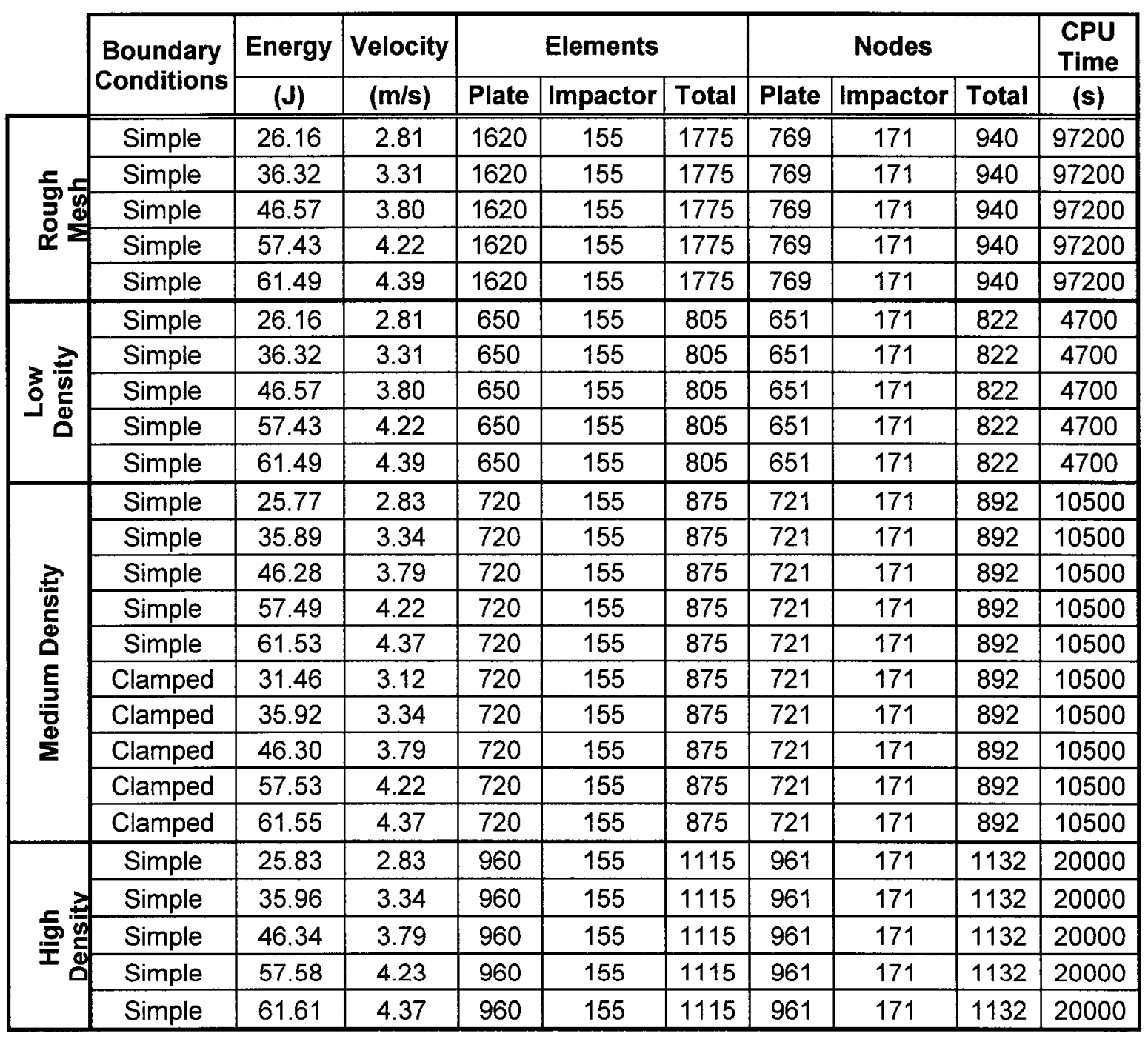




\subsection{Preliminary Simulation Results}

Figures 56 to 58 summarize the results of the aluminum impact simulations. As can be seen in Figure 56, the simulations predicted consistently lower absorbed energy than was measured in the experiments. However, all of the simulations predicted approximately the same values for absorbed energy, peak force and dent depth. Thus, mesh density and boundary conditions had little effect on the outcome of the simulations. Complete tabulated results are provided in Appendix E.

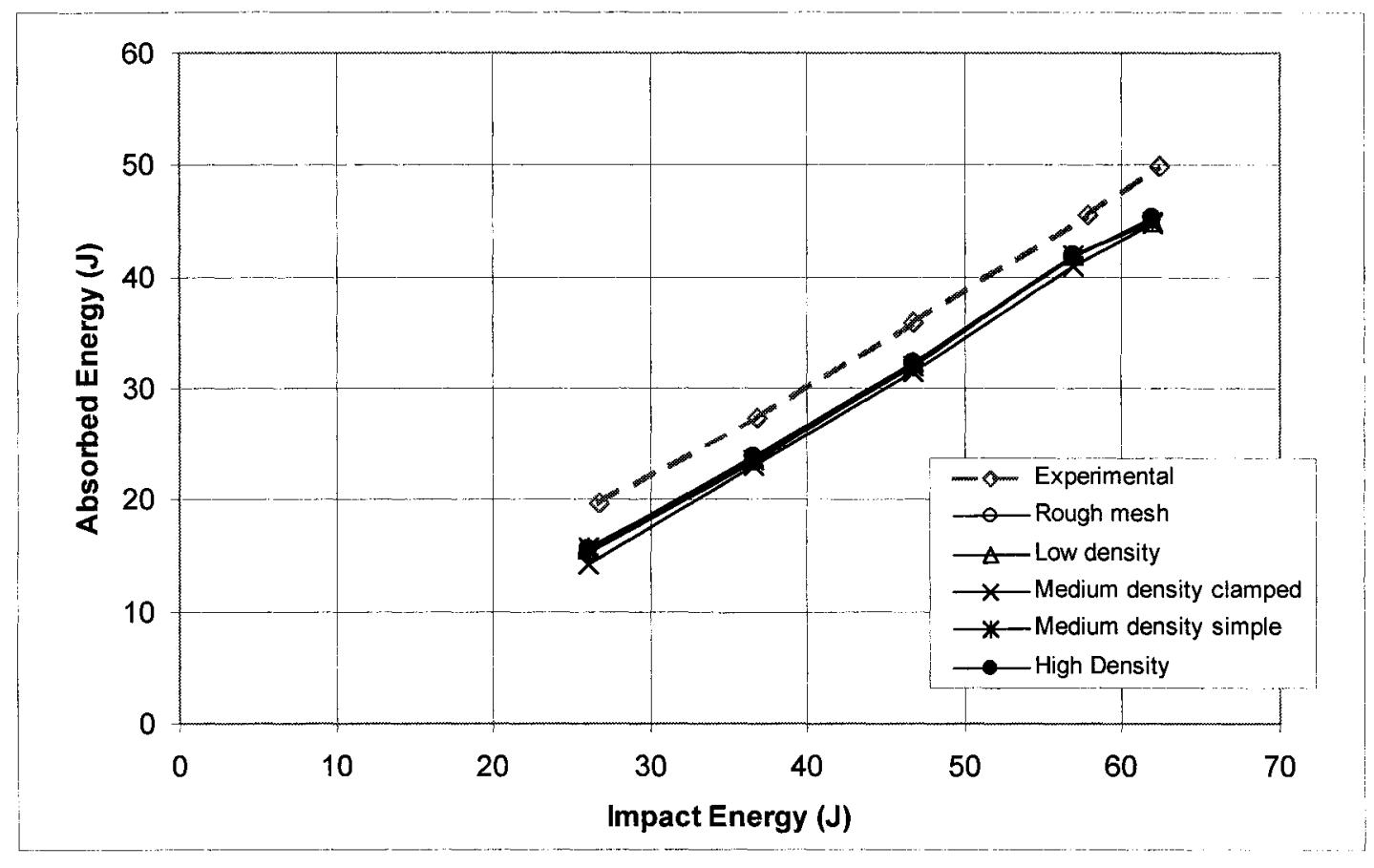

Figure 56: Absorbed energy versus impact energy for aluminum low-velocity impact experiments and simulations. 


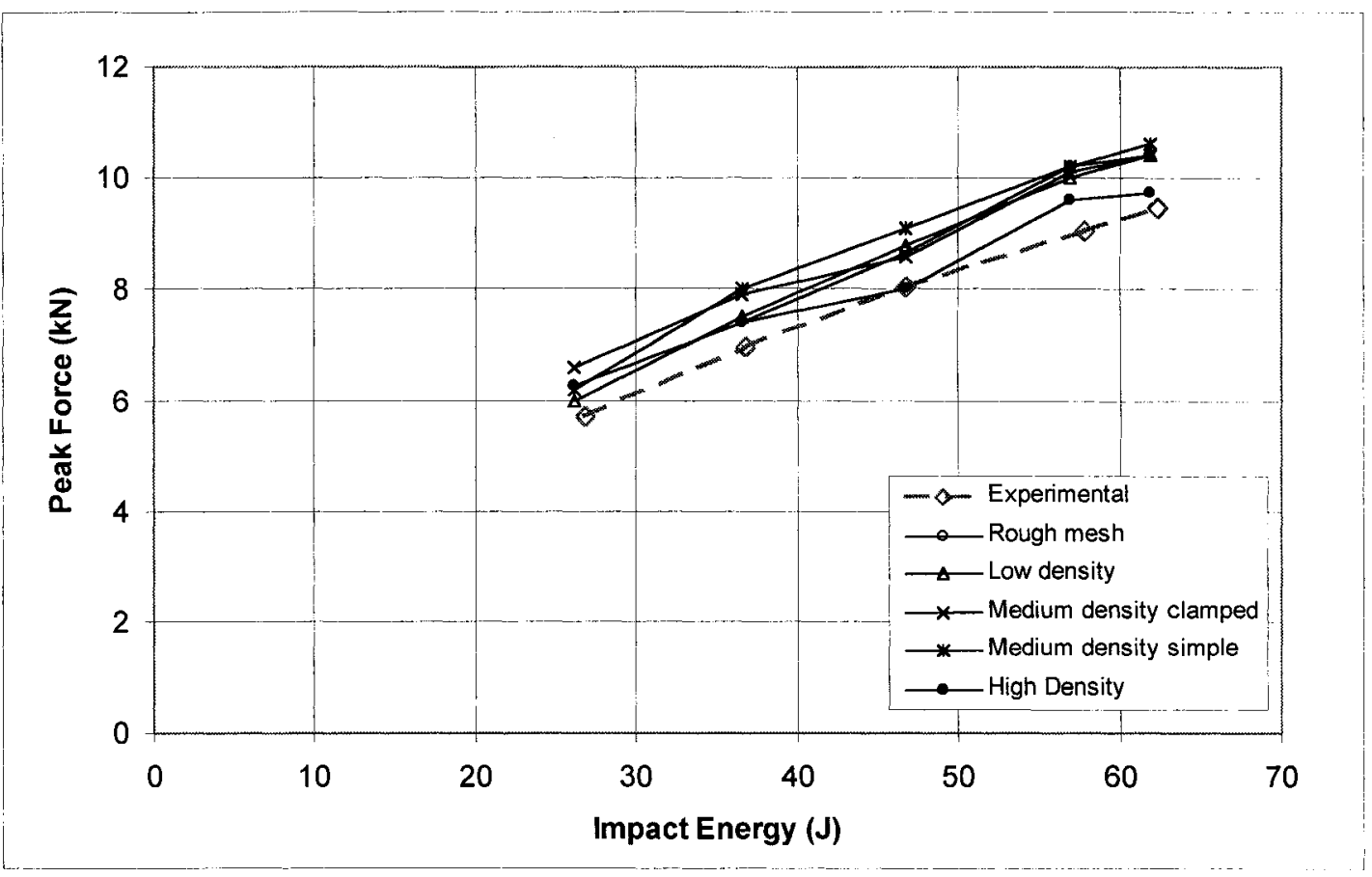

Figure 57: Peak force versus impact energy for aluminum low-velocity impact experiments and simulations.

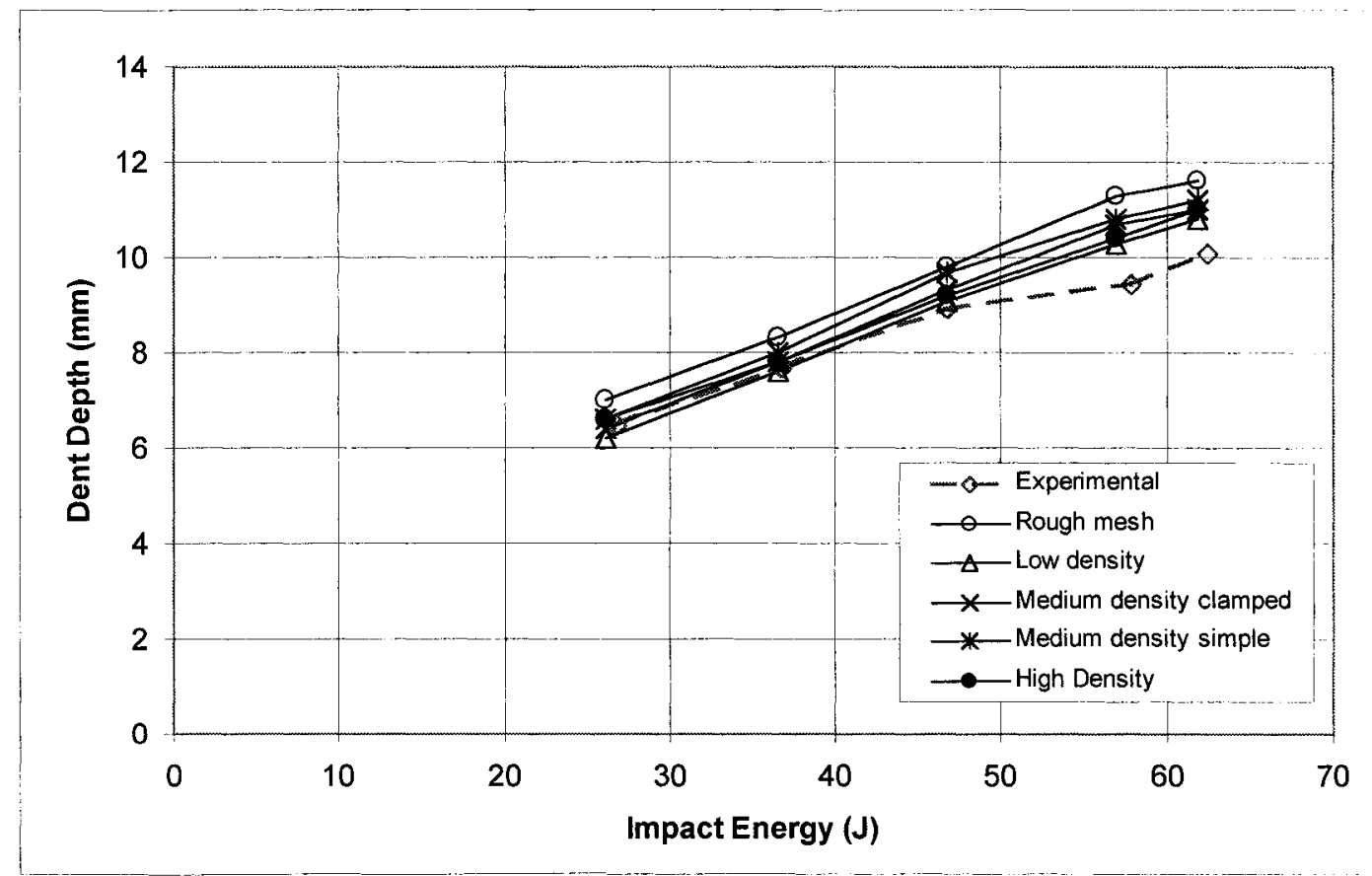

Figure 58: Dent depth versus impact energy for aluminum low-velocity impact experiments and simulations. 
The effect of increasing the number of elements on the CPU time is presented graphically in Figure 59. As can be seen, there is an approximately linear relationship between the number of elements and the $\mathrm{CPU}$ time required for the simulation. There is a decreasing dependence of the percent difference in predicted absorbed energy with increasing impact energy. However, there is no obvious relationship between the percent difference in absorbed energy and the number of elements. The difference in absorbed energy is calculated relative to the experimental values. Therefore, the amount of elements used in this study does not have an effect on the accuracy of the aluminum impact simulations.

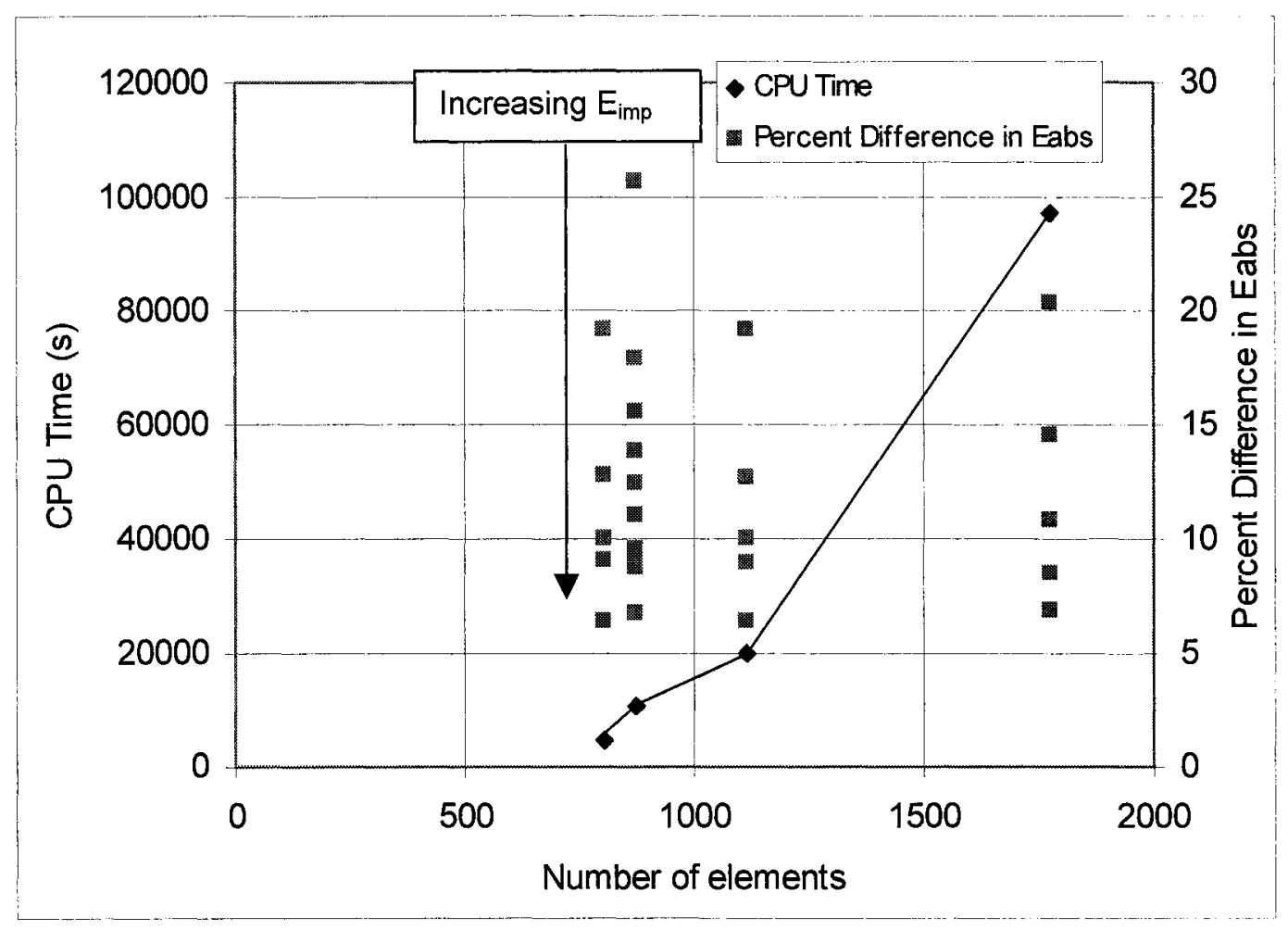

Figure 59: CPU time versus number of elements for preliminary simulations. 


\subsection{Comparison to Experimental Data}

The results of the aluminum simulations demonstrated the validity of the boundary condition assumptions made for the impact models. They also demonstrated the effect of the mesh density on the results, and provided familiarization training with LS-DYNA.

There was no effect of varying the mesh densities on the accuracy of the solution. However, there was a slight effect from the boundary condition. It was observed that the clamped panels absorbed less energy than the simply-supported panels. The clamped boundary condition does not allow as much deflection at the specimen edge as the simply-supported boundary condition. The overall stiffness of the specimen is increased and less elastic and plastic strain energy is absorbed.

There was a discrepancy of up to $20 \%$ between the simulated absorbed energy and the experimental values. The boundary conditions could be responsible for part of the discrepancy. To assume that the boundary is either clamped or simply-supported may not be accurate. As the panel is deformed during the impact event, there is some evidence that it slides out of the clamping fixture at the edges. Scrape marks are present at the periphery of the circular unsupported region in the impacted specimens described in Chapter 2. This would result in a boundary that is neither clamped nor simply-supported. However, for the present study a clamped or fully-fixed boundary condition will be assumed. 


\subsection{Final Simulations of Aluminum}

Following the conclusion of the preliminary modelling, a new mesh geometry was chosen for the final aluminum and GLARE simulations. This mesh was developed to eliminate the degenerated quadrilateral elements at the point of impact present in the radial meshes as shown in Figure 55.

\subsubsection{Simulation Parameters}

The mesh configuration (Figure 60) incorporates a region of equal sized square elements at the centre. This area was sized to correspond to the region of influence of the impactor observed in the experiments. For all of the simulations the impactor was modelled using thin-shell elements with the rigid material model (type 20) as shown in Figure 61. Friction between the steel impactor and the aluminum was assumed in the simulation. Literature values of 0.8 for the static coefficient and 0.5 for the kinetic coefficients of friction were employed (Herzberg, 1993).

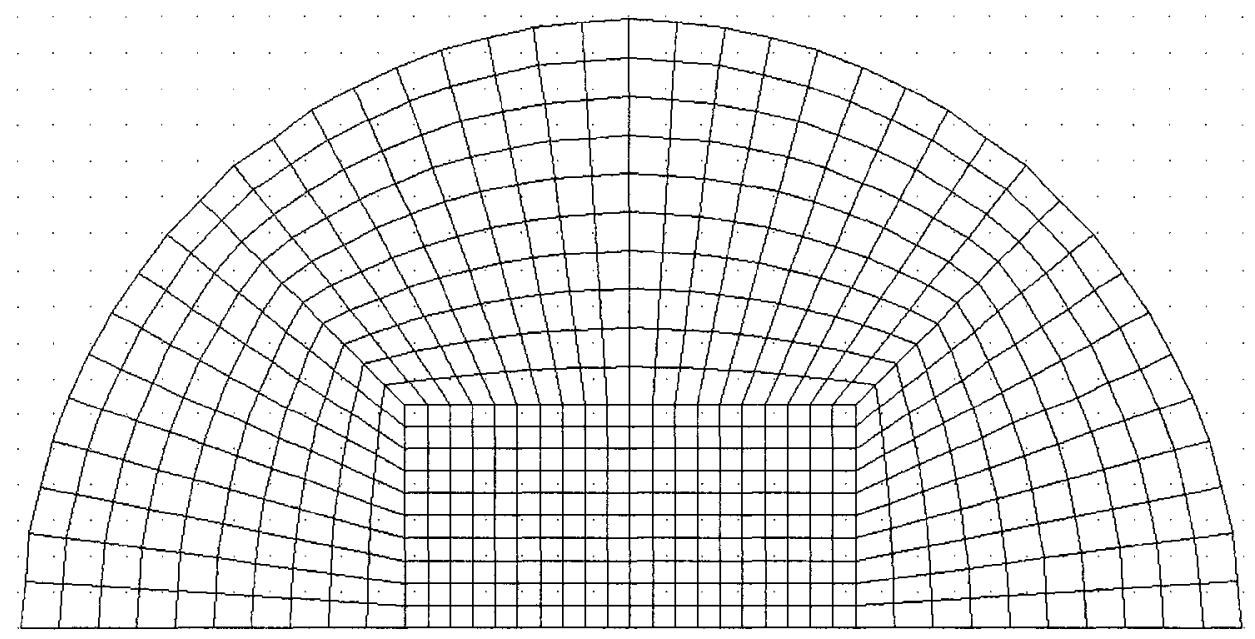

Figure 60: Half-symmetry mesh geometry. 

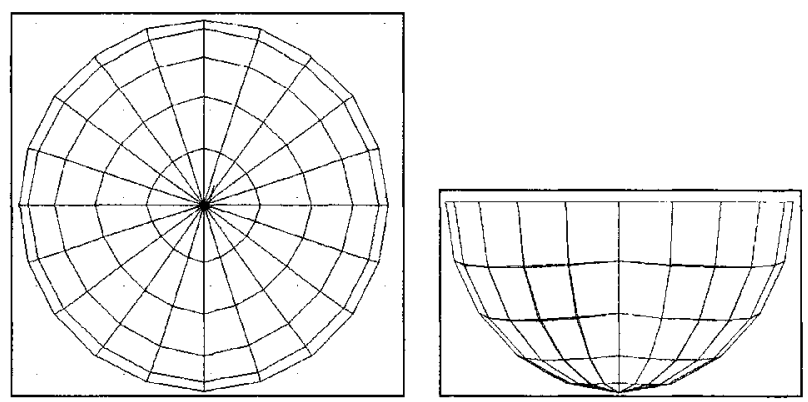

Figure 61: Impactor model geometry and mesh.

A description of the thin-shell, thick-shell and solid-brick element formulations can be found in Section 3.3. For the solid-brick simulations the maximum aspect ratio was approximately $8: 1$. The results of the simulations will be compared to each other and also to the experimental results. The basic aluminum properties employed for the finite element simulations were presented in Section 4.1. A detailed summary of the simulations carried out using monolithic 2024-T3 is presented in Table 17. This table also contains the final CPU times and number of cycles for each simulation. These simulations were performed on an Intel-based PC (750 MHz) running Redhat Linux version 7.1 with version $950 \mathrm{~d}$ of LS-DYNA.

Table 17: Summary of final 2024-T3 simulation parameters.

\begin{tabular}{|c|c|c|c|c|c|c|c|}
\hline ID Number & $\begin{array}{c}\text { Element } \\
\text { Type }\end{array}$ & $\begin{array}{c}\text { Energy } \\
\text { Level }\end{array}$ & $\begin{array}{c}\text { \# of } \\
\text { Elements }\end{array}$ & $\begin{array}{c}\text { \# of } \\
\text { Nodes }\end{array}$ & $\begin{array}{c}\text { CPU } \\
\text { Time } \\
(\mathbf{s})\end{array}$ & $\begin{array}{c}\text { Clock } \\
\text { Time }\end{array}$ & $\begin{array}{c}\text { CPU } \\
\text { Cycles }\end{array}$ \\
\hline 1-AL-PW-TN-25 & Thin & 25 & 1300 & 1342 & 425 & 850 & 31251 \\
\hline 2-AL-PW-TN-35 & Thin & 35 & 1300 & 1342 & 424 & 852 & 31250 \\
\hline 3-AL-PW-TN-45 & Thin & 45 & 1300 & 1342 & 426 & 1295 & 31239 \\
\hline 4-AL-PW-TN-55 & Thin & 55 & 1300 & 1342 & 431 & 1305 & 31220 \\
\hline 5-AL-PW-TK-25 & Thick & 25 & 1401 & 1342 & 3794 & 79901 & 120901 \\
\hline 6-AL-PW-TK-35 & Thick & 35 & 1401 & 1342 & 3840 & 15247 & 122387 \\
\hline 7-AL-PW-TK-45 & Thick & 45 & 1401 & 1342 & 3905 & 15702 & 123364 \\
\hline 8-AL-PW-TK-55 & Thick & 55 & 1401 & 1342 & 3911 & 15707 & 123234 \\
\hline 9-AL-PW-SO-25 & Solid & 25 & 1401 & 1342 & 2361 & 7330 & 164655 \\
\hline 10-AL-PW-SO-35 & Solid & 35 & 1401 & 1342 & 2455 & 7527 & 169068 \\
\hline 11-AL-PW-SO-45 & Solid & 45 & 1401 & 1342 & 5131 & 15408 & 172040 \\
\hline 12-AL-PW-SO-55 & Solid & 55 & 1401 & 1342 & 2358 & 8002 & 173615 \\
\hline
\end{tabular}




\subsubsection{Material Property Sensitivity}

Using the mesh geometry described in Section 4.6.1, three additional simulations were conducted to examine the effect of the aluminum properties on the predicted response of the aluminum panels. Figure 62 contains two additional stress-strain curves from the ASM handbook and MIL-HDBK-5H (ASM, 1990 and DoD, 1998) that were used to investigate the sensitivity of the impact simulations to the yield stress. Each of these sources is considered "correct"; yet as seen in the figure, there are differences between the stress-strain curves.

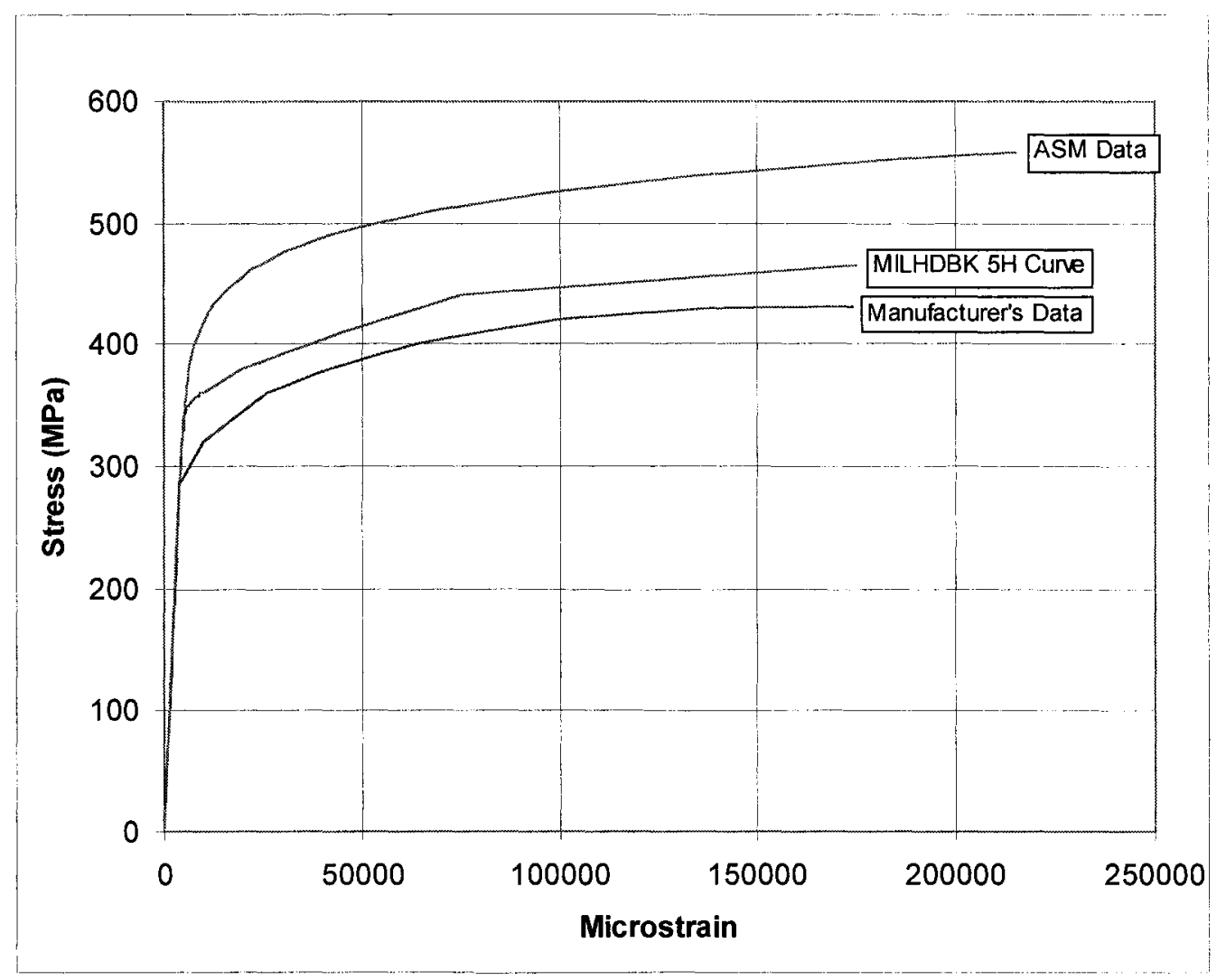

Figure 62: Stress-strain curves for 2024-T3 from various sources. 
The results of the material property sensitivity study are tabulated in Table 18 and displayed graphically in Figures 63, 64 and 65. The results show that the amount of absorbed energy increases with decreasing yield strength. This result is not surprising given that the panel simulated using the lower yield strength would suffer greater plastic deformation and thus absorb more kinetic energy. For a similar reason, the higher yield strength of the ASM material resulted in lower absorbed energy and higher peak force. The ASM panel underwent proportionally more elastic deformation than the other two panels and thus sustained a higher peak impact force.

This study revealed the effect of using material property data from a variety of sources. Due to the potential variations in the predicted impact results, it is important to use the manufacturer's data when it is available. Alternatively, tensile tests could be used to provide the required data. In all of the impact simulations in this thesis, the manufacturer's property data will be used.

Table 18: Material sensitivity study results.

\begin{tabular}{|l|c|c|c|c|}
\cline { 2 - 5 } \multicolumn{1}{c|}{} & Yield Strength & Absorbed Energy & Peak Force & Dent Depth \\
\cline { 2 - 5 } \multicolumn{1}{c|}{} & (MPa) & $(\mathbf{J})$ & $(\mathbf{k N})$ & (mm) \\
\hline ASM & 420 & 10.4 & 7.88 & 4.6 \\
\hline MILHDBK-5H & 345 & 12.4 & 7.17 & 5.4 \\
\hline Manufacturer & 285 & 14.4 & 6.89 & 6.1 \\
\hline Experimental & Not applicable & 18.8 & 5.70 & 6.2 \\
\hline
\end{tabular}




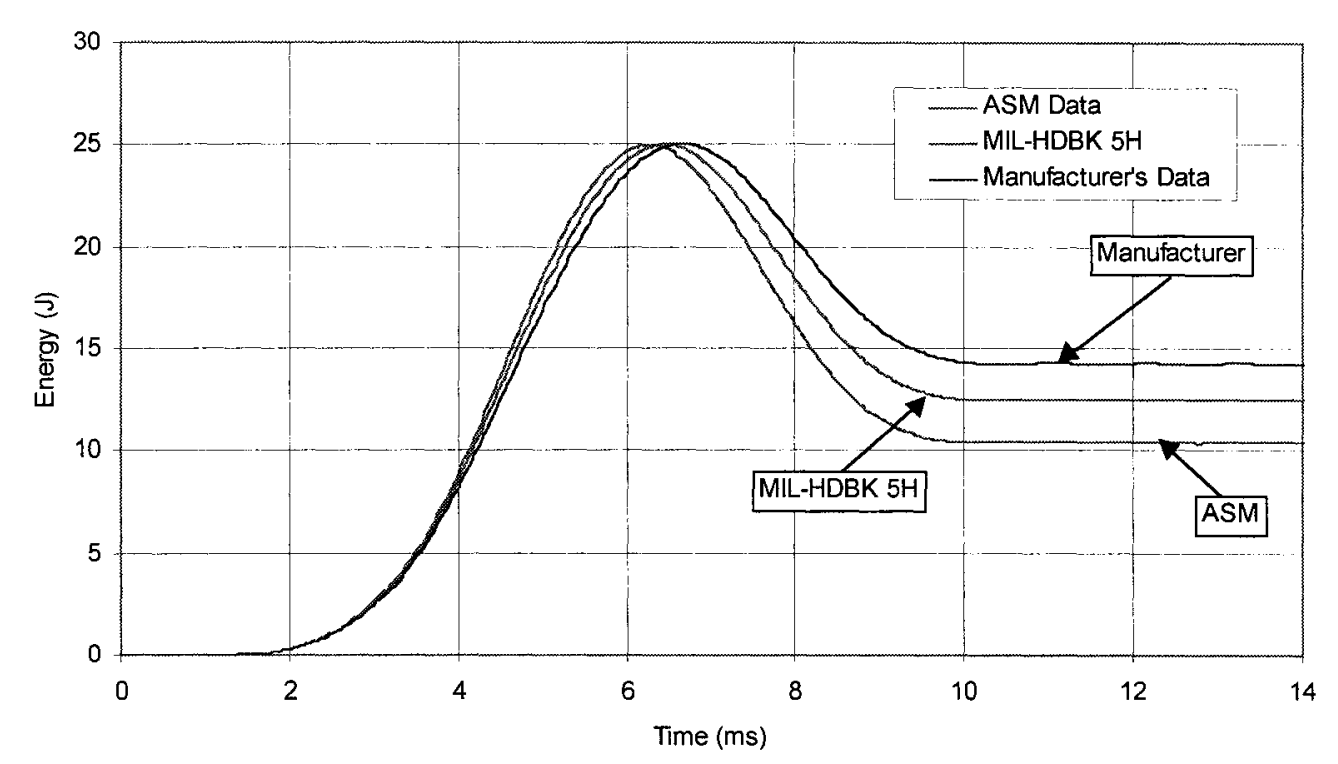

Figure 63: Impact energy results from material sensitivity study.

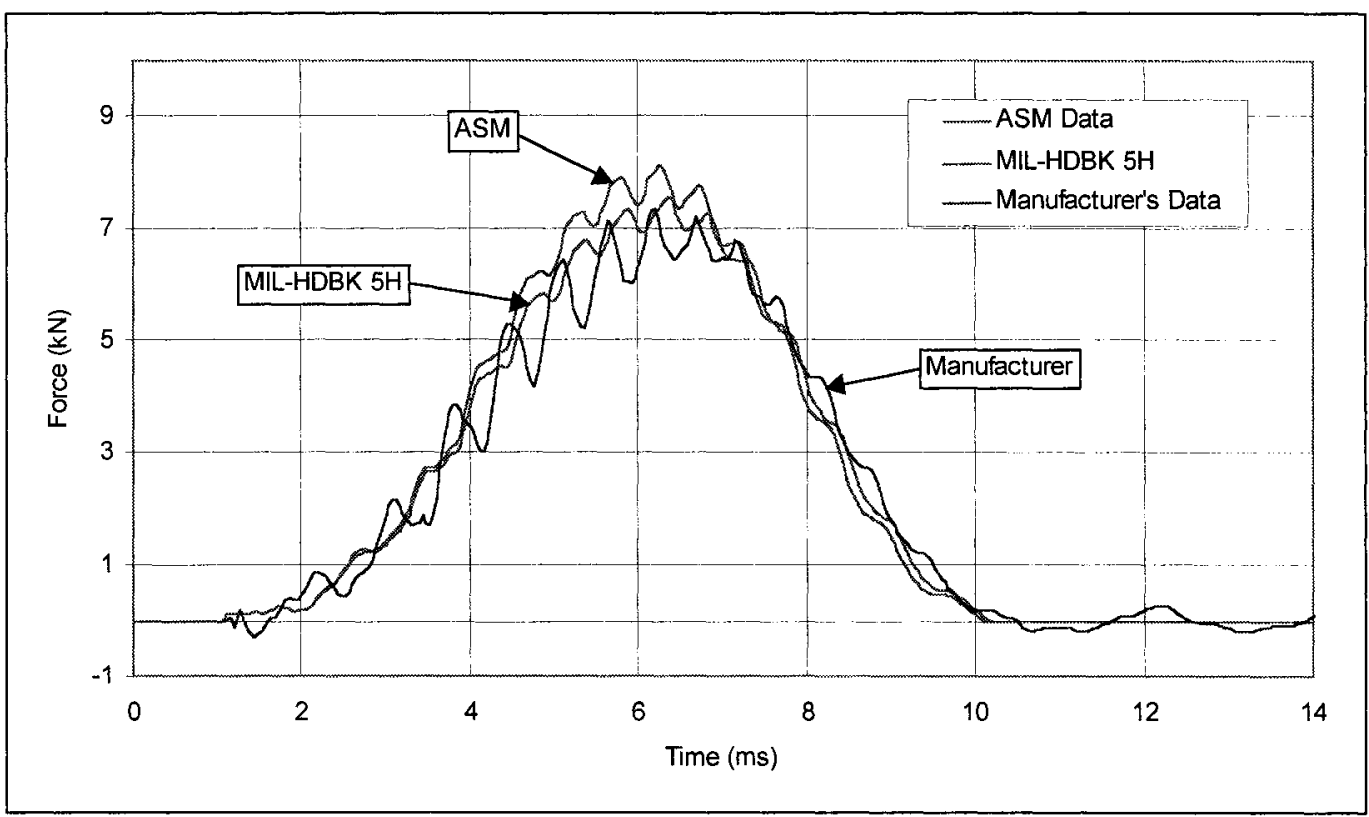

Figure 64: Peak impact force from material sensitivity study. 


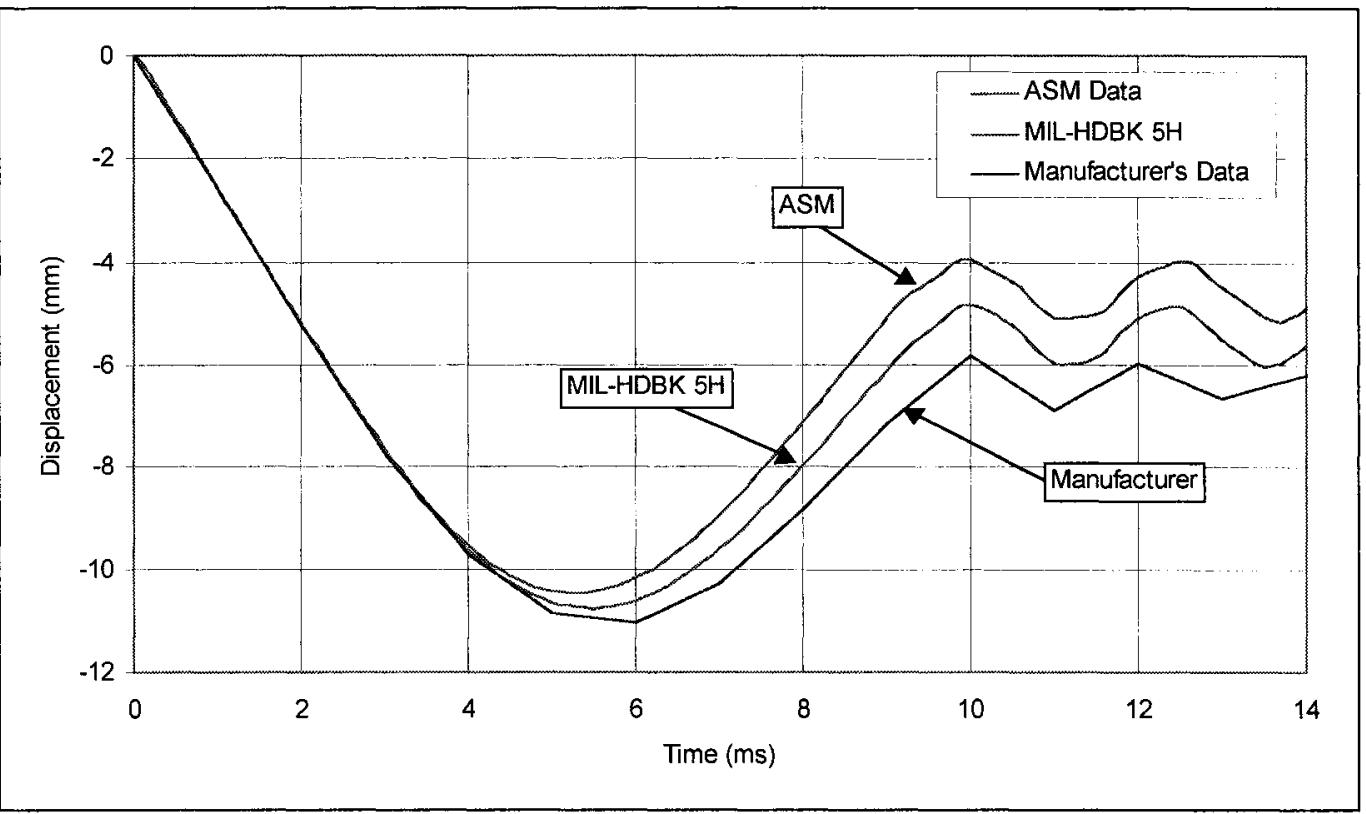

Figure 65: Dent depth results from material property sensitivity study.

\subsubsection{Results for the Final 2024-T3 Simulations}

The results of the impact simulations of aluminum are plotted versus the impact energy in Figures 66, 67 and 68. Complete tabulated results can be found in Appendix F. The final 2024-T3 simulation results were similar to the preliminary results described in Section 4.4. The amount of energy absorbed in the simulations was consistently less than that recorded in the experiments. This consistent deviation indicates a fundamental difference between the simulations and the experiments. The most likely sources of this error are the mechanical losses present in the drop tower impact experiments.

The predicted impact forces were higher than those measured in the experiments. The sources of this difference are the same as those that caused the difference in absorbed energy. Using rigid elements for the impactor and a perfectly fixed boundary condition for the specimen neglects the compliance of the drop tower support structure. The 
remainder of the drop tower structure was not modelled since it is the response of the aluminum panel that was of interest. The production of a fully detailed impact model could be a suitable follow-on project to study the losses in the structure.

The results obtained for the permanent deflection are interesting in that they show good agreement between the experiments and all the simulations except for the solidbrick simulations. The solid-brick simulations predicted permanent deflections $1.7 \mathrm{~mm}$ lower than those predicted using the other element types. Therefore, it was decided to use only thick-shell elements because of the advantages when modelling layered structures. These advantages include the designation of contact interfaces and the ability to model thin multi-layered structures. These results also confirmed the applicability of LS-DYNA to problems of low-velocity impact.

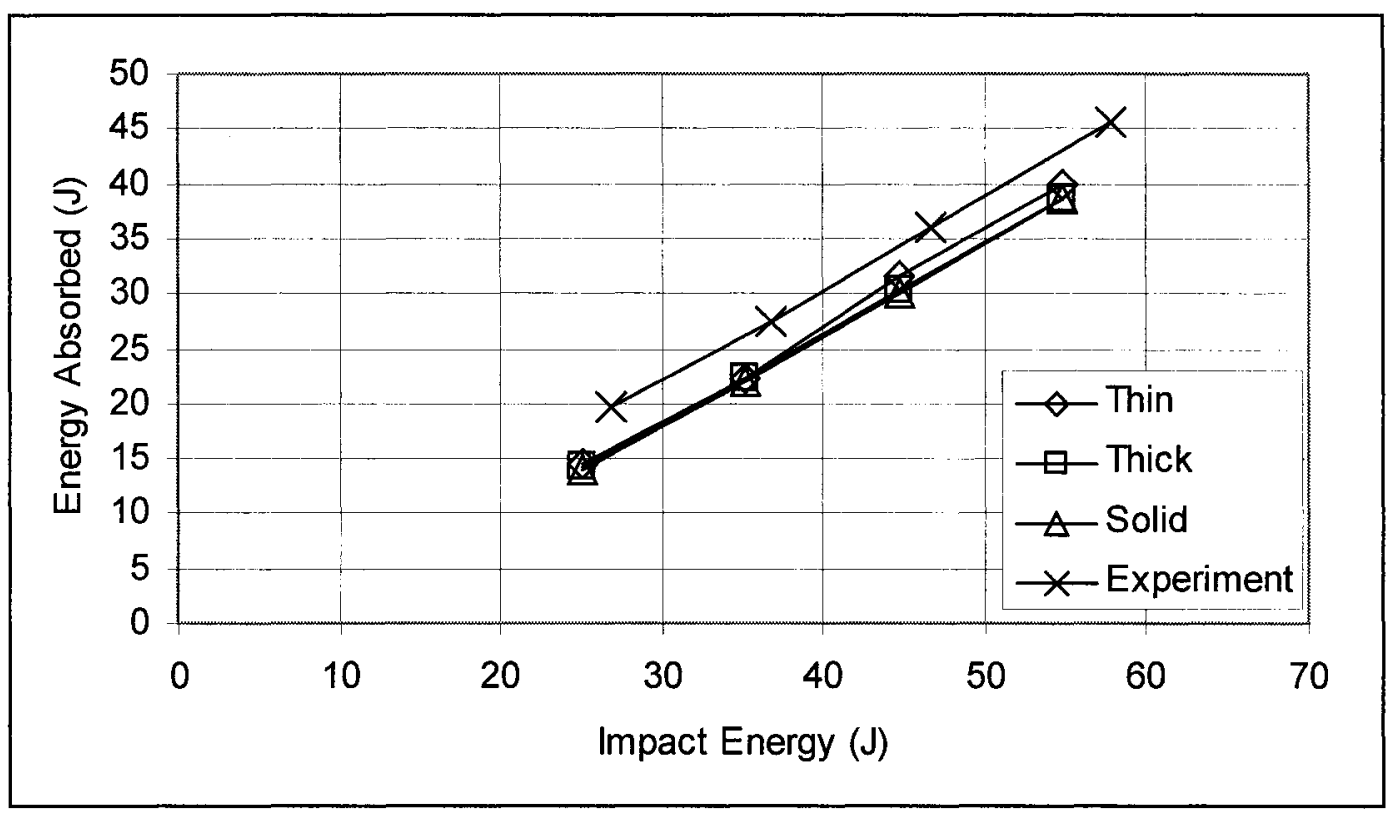

Figure 66: Absorbed Energy versus Impact Energy for the final impact simulations. 


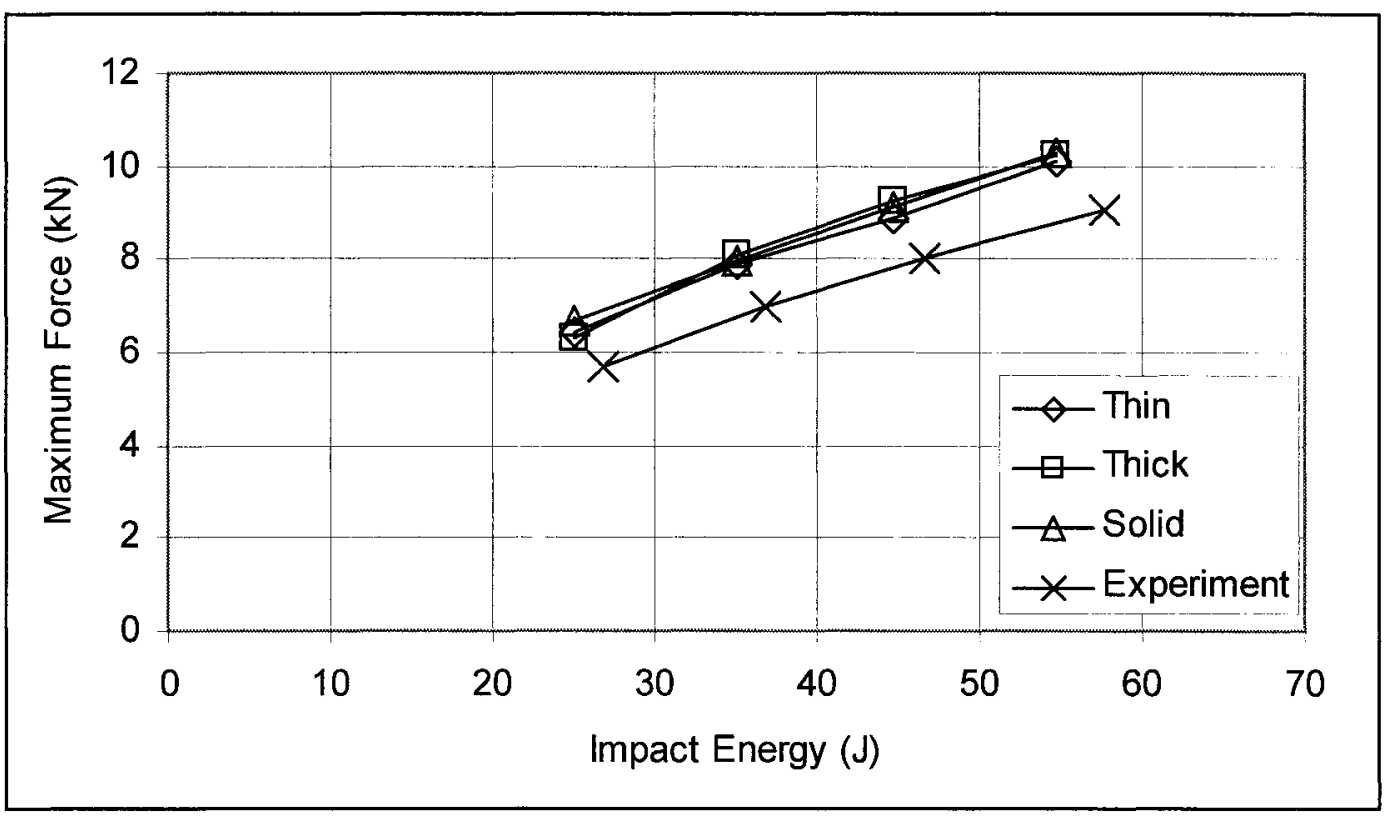

Figure 67: Maximum Force versus Impact Energy for the final simulations.

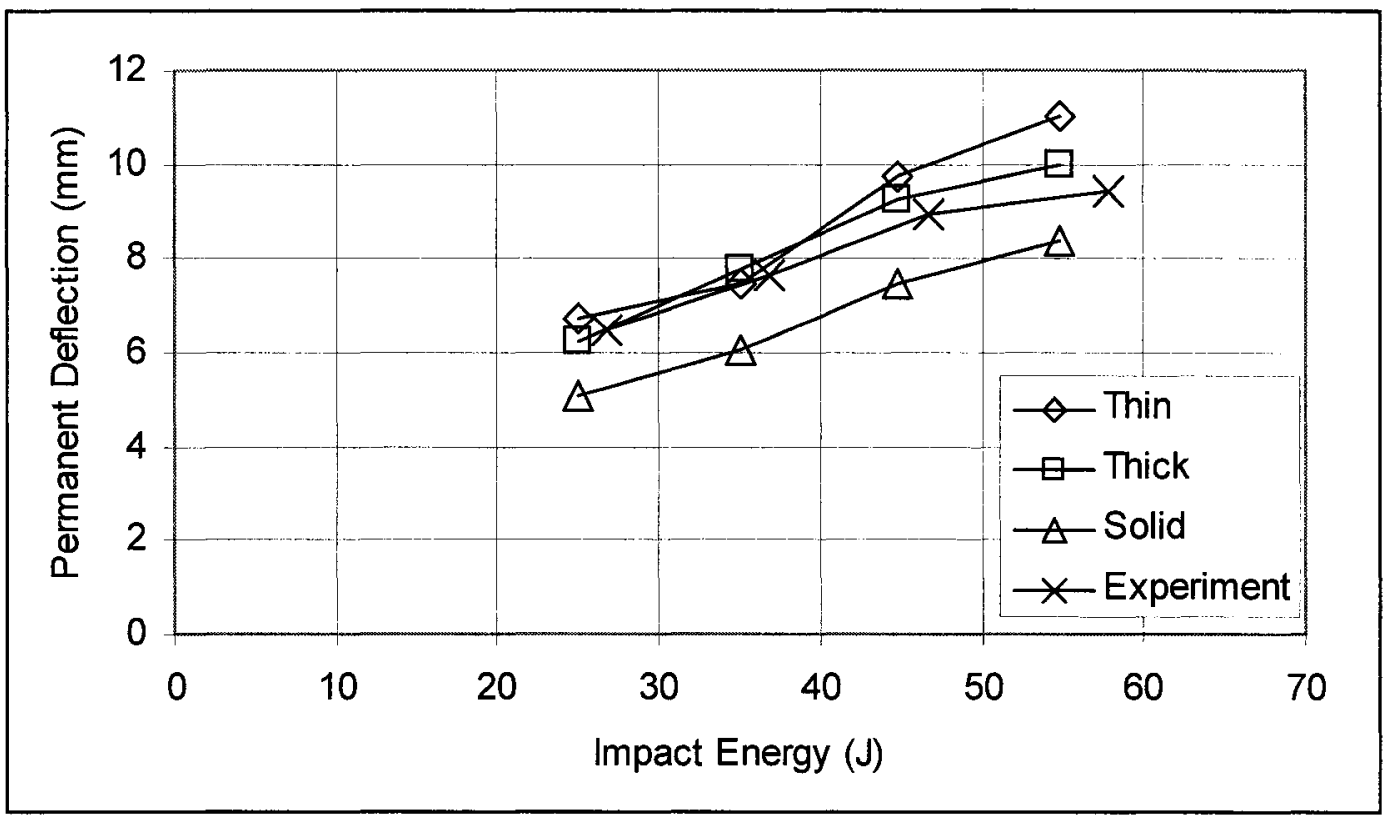

Figure 68: Permanent Deflection versus Impact Energy for the final impact simulations. 


\section{CHAPTER 5}

\section{DELAMINATION DAMAGE}

\section{CHARACTERIZATION}

As described in Section 3.2, one of the components required to successfully simulate the damage formed in GLARE laminates is an accurate model of delamination initiation and growth in the panels. Manufacturer's strength data can be used for delamination initiation with the tiebreak-interface in LS-DYNA. However, experiments are required to characterize the post-delamination behaviour. Section 5.1 presents a review of delamination characterization techniques for FMLs. The results of a series of double cantilever beam (DCB) tests are then presented in Section 5.2. 


\subsection{Review of Delamination}

\section{Characterization of FMLs}

Mode I delamination damage has been identified as an important damage mode in impacted FMLs. Two groups of researchers have characterized delamination damage in FMLs previously. Vries et al. (1999) conducted an experimental investigation into the delamination behaviour of spliced fibre-metal-laminates. This study was part of the BRITE-EURAM-III project investigating concepts for next-generation European passenger aircraft. They examined several splice concepts and subjected test coupons to cyclic loading to measure the resistance to delamination growth, R. Two reasons were given for using this parameter, based on strain energy release, over others based on tensile and interlaminar strength:

1. Mixed mode delamination is easy to characterize with the single resistance parameter as opposed to two critical shear and tensile stresses.

2. Neither the stress concentration at the delamination tip nor the distribution of stresses over the thickness of the laminate have to be considered.

It was found that the delamination growth rates are independent of the delamination size.

Vlot and van Ingen (1998) studied delamination in unidirectional ARALL-2, ARALL-3 and GLARE-1 under static and dynamic loading. Each of the laminates was tested in the as-cured condition and in a post-stretched state ( $0.4 \%$ permanent stretch). As previously discussed, in as-cured FMLs there is a residual tensile stress-state in the aluminum layers. This can lead to reduced fatigue resistance in the panels. Some panels 
of each laminate were also subjected to a second curing cycle following the stretching operation to simulate the final bonding step for an airframe component. Double cantilever beam (DCB) tests and end notch flexure (ENF) tests were used to study the $G_{I C}$ and $G_{I I C}$ energy release rate, respectively. The dynamic tests were conducted with a specimen that simulated the edge of a bonded FML doubler. This configuration is seen in aircraft that employ bonded doublers around doors and windows.

The post-stretch operation had a positive influence on the mode I static delamination resistance in ARALL and no influence on the mode II delamination resistance. In GLARE laminates the post-stretching had no influence on the mode I static delamination resistance and only a small positive effect on the mode II resistance. It was also found that the GLARE panels exhibited significantly higher overall delamination resistance than ARALL under static conditions. Equations based on classical beam bending theory combined with rotation of the two beams at the delamination tip in the DCB specimen were developed to calculate the energy release rates. A series of DCB tests using the specimen shown in Figure 69 were carried out.
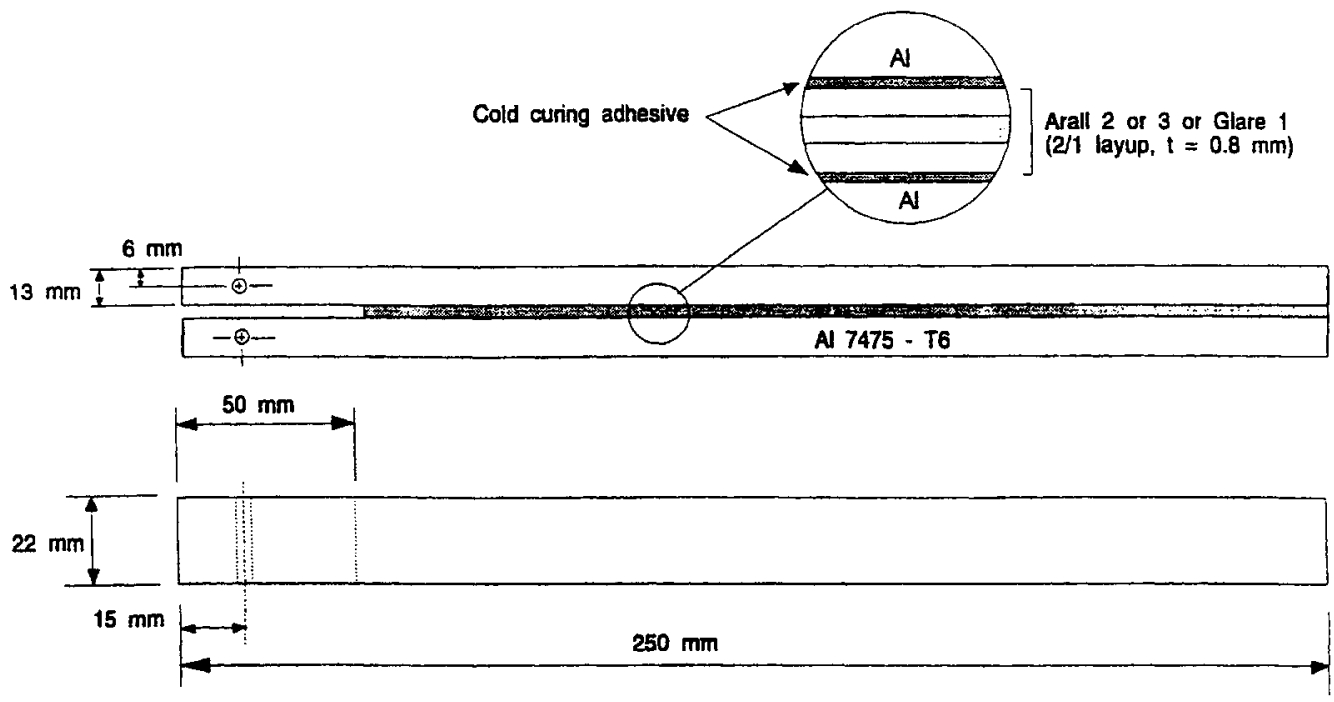

Figure 69: DCB specimen (reproduced from Vlot and Ingen, 1998). 
Aluminum beams of the same composition as the aluminum used in the fibremetal lay-up of GLARE were used to support the FML coupon. The DCB specimens were loaded at constant displacement until delamination occurred. The displacement was stopped immediately and the crack length was then marked on the specimen edge. This process resulted in a load-displacement curve for each specimen.

The test method used by Vlot and van Ingen is similar to the composite DCB test methods discussed by Hashemi et al. (1989), Whitney (1989) and Russell and Street (1983). However, Vlot and van Ingen did not employ the same basic loading method. When loading the specimens, the crack was allowed to propagate for a set interval before unloading. However, the other researchers used an initial crosshead displacement that was held during the growth of the delamination. When the propagation of delamination ceased, the load was removed. No reason for the difference in loading method was indicated. 


\subsection{DCB Characterization of GLARE}

To support the development of the damage modelling methodology, a series of DCB tests was conducted using several types of GLARE. These tests were intended to provide crack opening stress versus displacement data for the LS-DYNA impact model. Coupons of GLARE-3, -4 and -5 were selected for the DCB tests; four samples of each for $0^{\circ}$ fibre orientation and four GLARE- 5 coupons in the $90^{\circ}$ fibre orientation (Table 19).

Table 19: Experimental test matrix.

\begin{tabular}{|c|c|}
\hline Material Type & $\begin{array}{c}\text { Number } \\
\text { Tested }\end{array}$ \\
\hline GLARE-3-2/1 & 4 \\
\hline GLARE-4-2/1 & 4 \\
\hline GLARE-5-2/1 $\left(0^{\circ}\right)$ & 4 \\
\hline GLARE-5-2/1 $\left(90^{\circ}\right)$ & 4 \\
\hline
\end{tabular}

\subsubsection{Preparation of Test Specimens}

The test article design was based upon that of Vlot and van Ingen (1998) as shown in Figure 70. To ensure that a crack would initiate in the GLARE coupon rather than the bond line between the coupon and the aluminum bar, a notch was introduced using a 0.15 $\mathrm{mm}$ thick diamond saw blade. The notch was protected with Teflon tape to ensure that adhesive would not overflow into the notch. The room temperature-cure adhesive known as DP-420 from $3 \mathrm{M}$ was used to bond the specimens to the adherends. 


\subsubsection{Test Procedure}

The specimens were prepared as shown in Figure 70. Each specimen was tested using an MTS hydraulic load frame under displacement control through pins inserted in the holes near the end of the specimen. They were then loaded quasi-statically at a rate of $5 \mathrm{~mm} / \mathrm{min}$ under displacement control until the first evidence of cracking occurred on the load curve. This crack length was measured and the load was reduced to zero. Each specimen was again loaded until the crack began to grow again. The crack was allowed to grow to another arrest point. This procedure was repeated until a final overall crack length was reached.

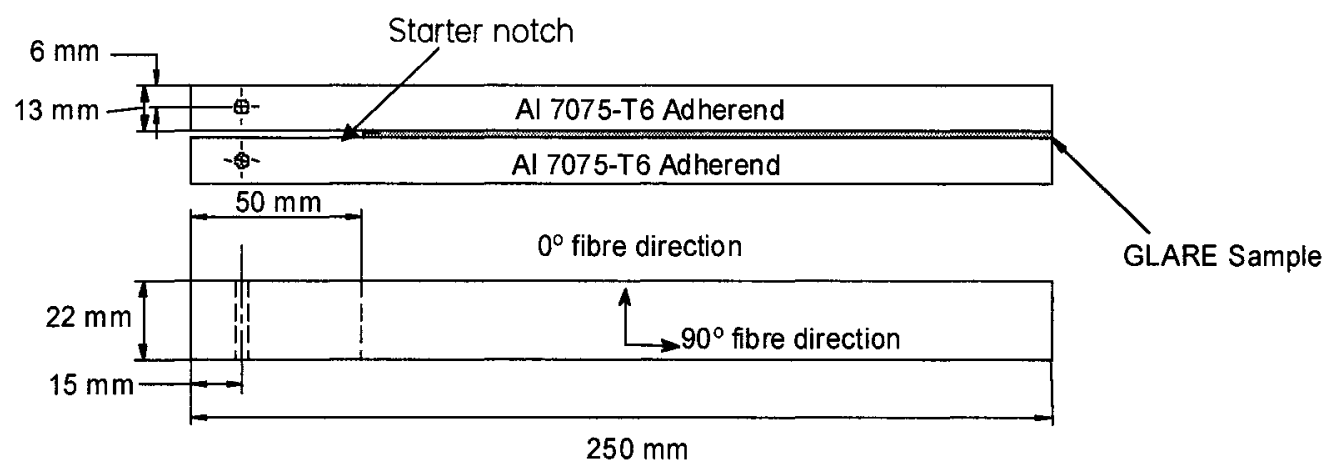

Figure 70: GLARE DCB specimen configuration.

\subsubsection{Delamination Test Results}

A sample load versus crosshead displacement curve is shown in Figure 71. This particular load-displacement curve was considered acceptable since a total of 7 crack length measurements were possible. Figure 72 shows an unacceptable crack growth curve from a GLARE-3 specimen. Only two measurements were possible using this specimen and then the specimen failed in the bondline between the laminate and the aluminum bar. The delamination failed to propagate any further. 


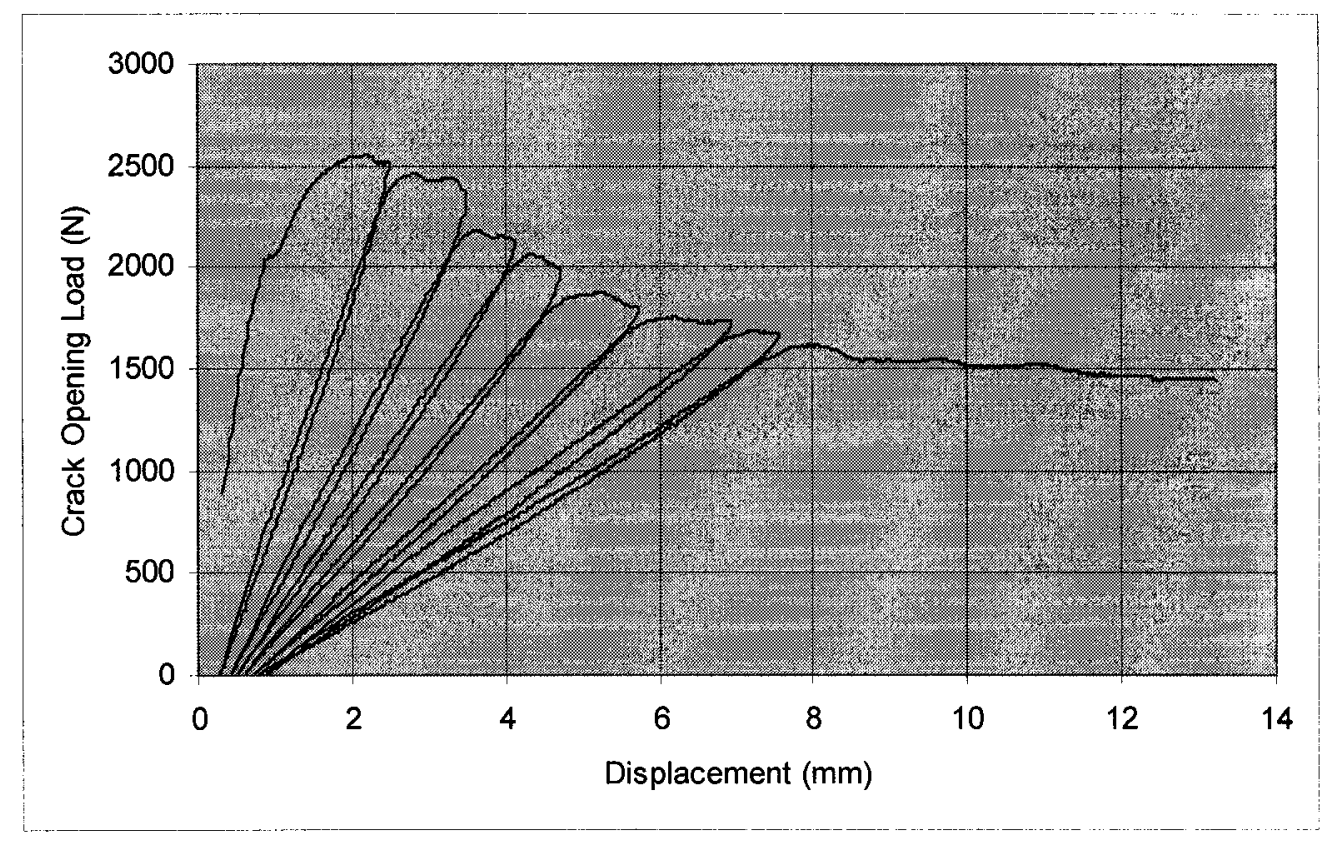

Figure 71: Acceptable load curve from a GLARE-5 DCB test.

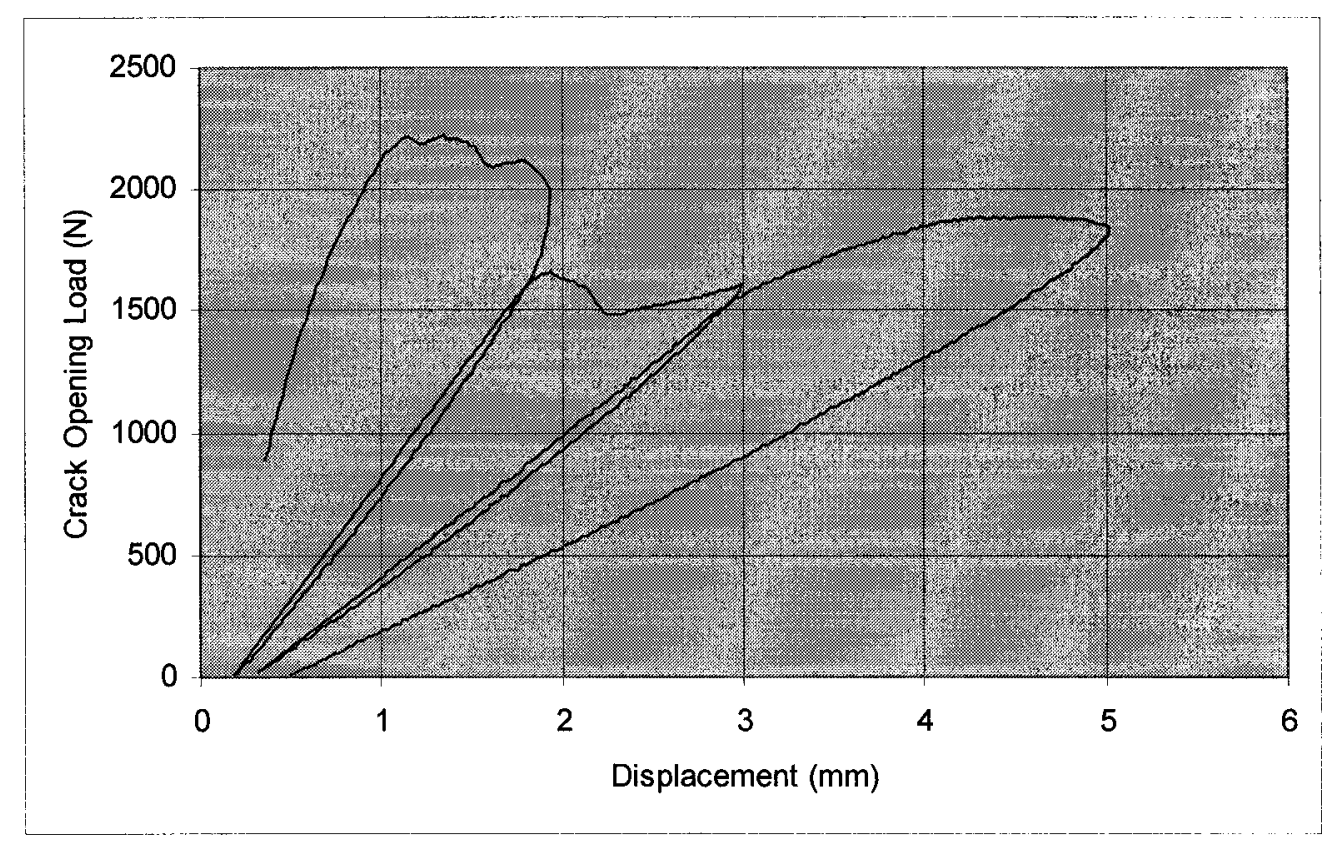

\section{Figure 72: Unacceptable load curve from a GLARE-3 DCB test.}

Extensive fibre bridging was noted during examinations of the fracture surface of the specimens (Figure 73 and Figure 74a). The delamination cracks propagated in prepreg layers with the fibre oriented parallel to the delamination direction. Each of the 
three types of GLARE exhibited the same behaviour. This indicates that the "path of least resistance" through the prepreg layer is parallel to the fibres rather than perpendicular. The fibres pull away from the matrix and produce the fracture surface shown in Figure 73.
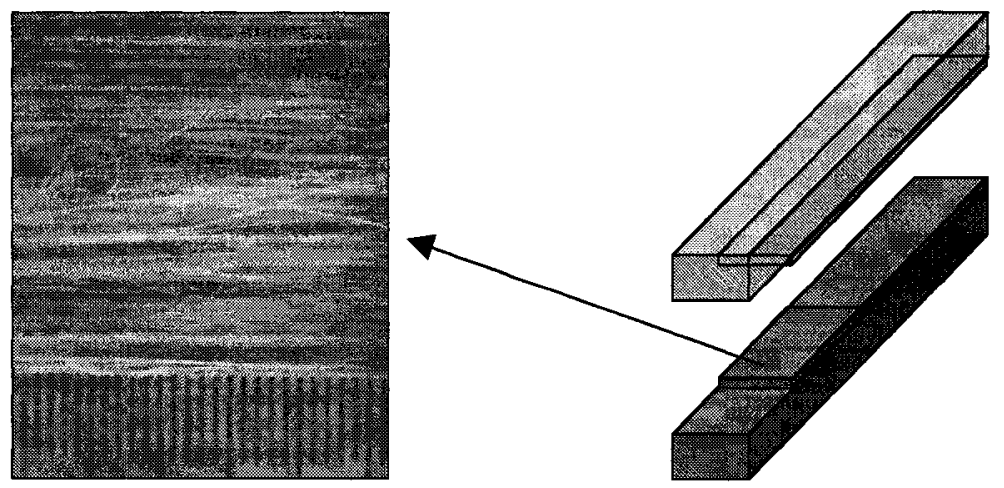

Figure 73: Optical micrograph of the failure surface in a typical DCB specimen.

Although the geometry was different between the DCB specimens and the impacted specimens, the damage modes were similar. Therefore the DCB results can be applied to the impact simulations. A comparison between the DCB and impact specimen delamination fractures is shown in Figure 74. As can be seen in this figure the fibre bridging in both types of specimens are similar.

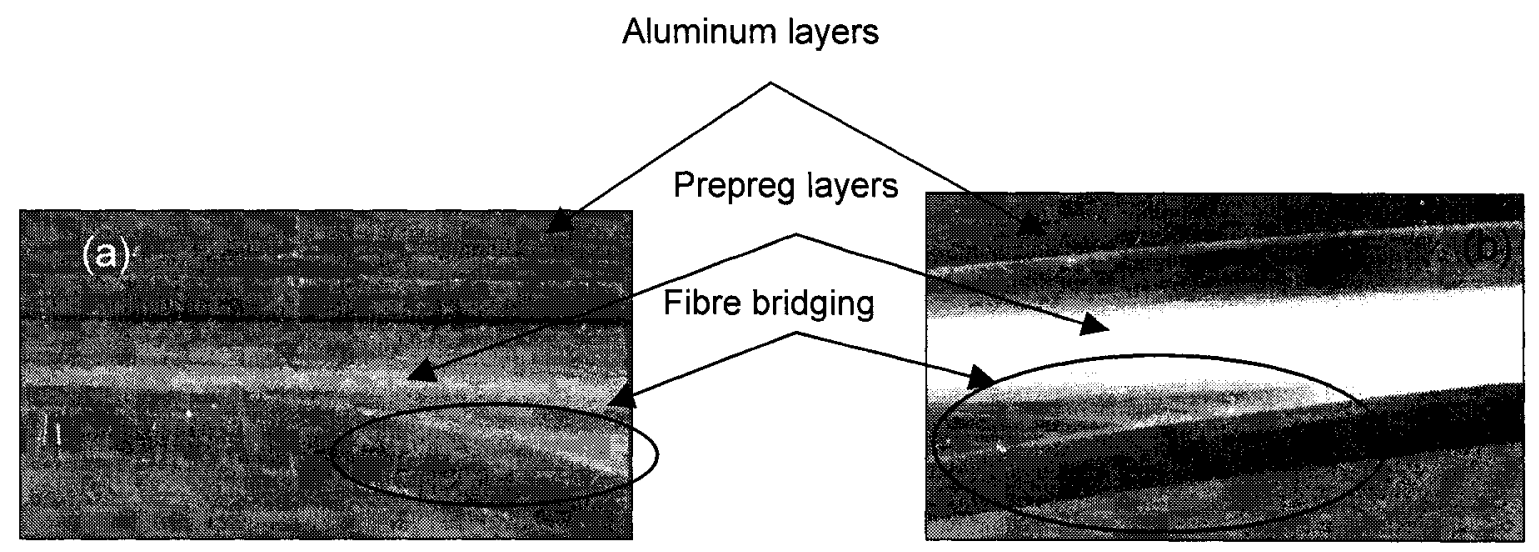

Figure 74: (a) Fibre bridging in a typical FML DCB specimen and (b) in an impacted FML specimen. 


\subsubsection{Application of the DCB Test Results in LS-DYNA}

As described in Section 3.3.5 it is possible to incorporate a piecewise linear stressdisplacement curve for modelling ply separation in LS-DYNA during delamination failure. Upon more detailed examination of the DCB test data it was determined that the load-displacement curves generated during the testing could not be directly incorporated into LS-DYNA. LS-DYNA requires the stress at the interface rather than the overall load, as was generated by the DCB tests. It was not possible to convert the loaddisplacement data from the experiments into stress-displacement curves. These tests, however, did provide useful information into the overall delamination behaviour in the laminates. A new specimen may be required to provide the appropriate data. A finite element package specifically suited for modelling the stress singularity at the tip of the delamination could also be used to determine the stresses for a given displacement. 


\section{CHAPTER 6}

\section{DEVELOPMENT OF A CDM-}

\section{BASED SUBROUTINE}

This chapter describes the development of a user-defined subroutine for the prepreg layers in the GLARE laminates. A review of continuum damage mechanics $(\mathrm{CDM})$ is presented in Section 6.1. Continuum damage mechanics is a branch of materials science that has been widely used to predict the degradation and failure of engineering materials in many applications. The application of one CDM-based approach within LS-DYNA is discussed in Section 6.2. The validation of the model using coupon simulations is given in Sections 6.3 and 6.4. 


\subsection{Review of Continuum Damage}

\section{Mechanics for Composites and FMLs}

In his seminal work, Kachanov (1958) described the cumulative effect of the deterioration of a material through the formation of microcracks by using a field variable. A dimensionless scalar field variable $\varphi$ is used to describe the state of the material. In an undamaged material this quantity is $\varphi=1$ and in a completely damaged material it is $\varphi=0$. As this theory was originally applied to creep-rupture, Kachanov developed a law in which $\varphi$ varied with time at elevated temperatures. The complementary quantity, $d=1-\varphi$, was defined to denote the level of damage or deterioration.

This theory was later expanded and applied to numerous other physical situations. These include fatigue damage, thermomechanical fatigue and damage in composite materials. Continuum damage mechanics can be applied to both the lamina and the interface between the laminae in composites. Lemaitre $(1987,1992)$ summarizes the original development of Kachanov's theory and subsequent additions and improvements. To illustrate the basic concept of the damage variable, Figure 75 shows a simple uniaxial specimen under a tensile load, $F$, in the normal direction, $\vec{n}$. The large volume is referred to as the Representative Volume Element (RVE) and is sized to represent an average of the microprocesses, but must also be small enough that high gradients in the material are not excessively smoothed out. This scale is approximately $1 \mathrm{~mm}^{3}$ for composites (Lemaitre, 1992). Within the specimen there is a plane perpendicular to $\vec{n}$.

Within the element there is $\delta S$, which is the area of intersection between the plane and the 
RVE. There is also have $\delta S_{D x}$, which is the effective area of intersection between all microcracks and cavities that lie in $\delta S$.

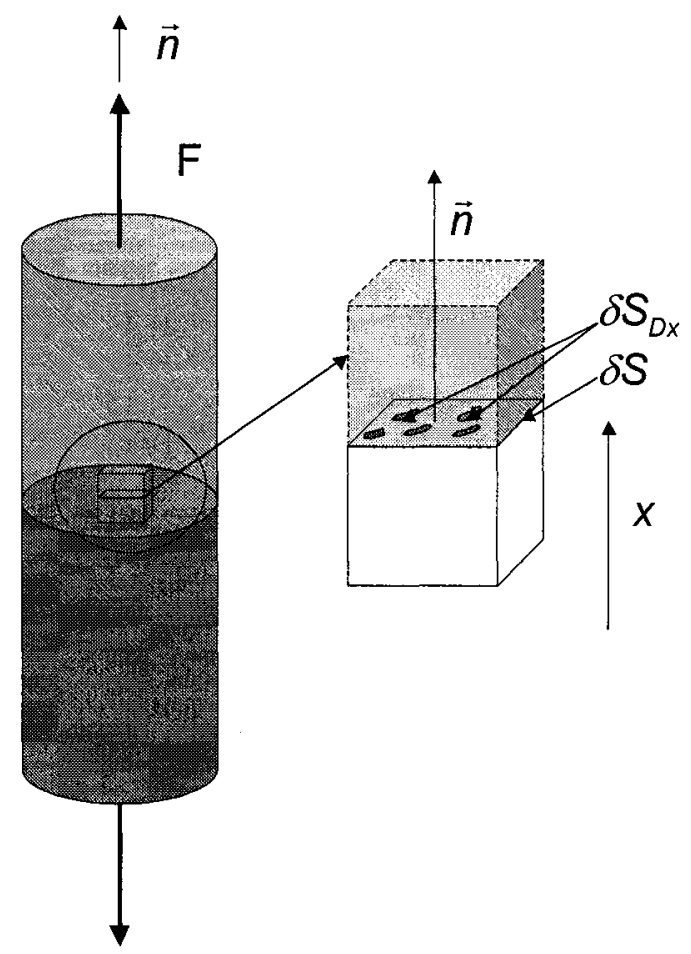

Figure 75: Representation of the micro-meso scale definition of damage (adapted from Kachanov, 1958 and Lemaitre, 1992).

There is an amount of damage associated with the RVE in the direction of $\vec{n}$ and at the point $x$ that is given in Equation 41 .

$$
d(\vec{n}, x)=\frac{\delta S_{d_{x}}}{\delta S}
$$

The final failure plane is that which contains the most damage. This is found by examining all planes along the $x$ direction as shown in Equation 42 .

$$
d_{(\bar{n})}=\operatorname{Max}_{(x)}\left[\mathrm{d}_{(\bar{n}, x)}\right]
$$

Thus, the co-ordinate $x$ disappears and the final relation for the damage is given by Equation 43. 


$$
d(M, \vec{n})=\frac{\delta S_{d}}{\delta S}
$$

The value of $d$ is bounded by 0 and 1 , where 0 represents completely undamaged material and 1 represents completely failed material. In reality the specimen will fail before the value of $d$ reaches 1 through a process of material instability that leads to rupture at the microscopic level.

There is typically a critical value of damage, $d_{c}$, that determines when failure will occur. In a purely brittle fracture the damage will be $d_{c} \sim 0$ and in a pure ductile fracture it will be $d_{c} \sim 1$. This amount of damage can be related to a critical value of the effective stress, $\sigma_{\infty}$, in the material as follows in Equation 44 .

$$
\tilde{\sigma}=\frac{\sigma}{1-d_{c}}=\sigma_{\infty}
$$

In practice the value of $d_{c}$ is closer to 0.5 , as shown by Ladevèze and Le Dantec (1992) for fibre reinforced composites. It is possible to approximate the critical stress, $\sigma_{\infty}$, with the ultimate stress, $\sigma_{u}$, which is smaller and to arrive at an estimate for $d_{c}$ as shown in Equation 45.

$$
\left(d_{c}\right)_{e s t} \approx 1-\frac{\sigma}{\sigma_{u}}
$$

For example, in a uniaxial monotonic stress test, $\sigma$ is equal to the rupture stress of the specimen. This test can then define a value of $d_{c}$, which is then considered a material characteristic.

It has been shown (Lemaitre, 1992) that "any strain constitutive equation for a damaged material may be derived in the same way as for virgin material except that the usual stress is replaced by the effective stress." This concept of effective stress is very 
important for the application of damage mechanics. A consequence of this principle is that the elastic modulus can be measured to determine the amount of damage in a specimen. By extension, other similar material properties, such as the flexural modulus or the shear modulus, could also be measured. There is thus an influence of damage on the elastic response of the material:

$$
\varepsilon_{e}=\frac{\sigma}{E(1-d)}
$$

where $\varepsilon_{\mathrm{e}}$ is the elastic strain. For the Young's modulus, the relationship with damage is given by Equation 47 .

$$
d=1-\frac{E}{E^{o}}
$$

This allows the direct measurement of the accumulation of damage through a series of cyclic tensile loading-unloading tests.

The damage constitutive equations are determined using a thermodynamic framework, in terms of the strains used in elasticity, plasticity and viscoplasticity. Through this method the coupling between all the different physical phenomena is obtained automatically. This allows the accumulation of damage to reduce the load carrying capacity of the material, resulting in a reduction in the modulus of the material.

The thermodynamic foundations of continuum damage mechanics have been described by numerous researchers since Kachanov (Lemaitre, 1987, Chaboche, 1988, Maugin, 1992 Germain et al, 1983, Coleman and Gurtin, 1967 and Krajcinovic, 1983). Chaboche (1988) and Lemaitre (1992) stated that the damage strain energy release rate is a function of the change in free energy density as shown in Equation 48 . 


$$
\bar{Y}=\rho \frac{\partial \psi}{\partial d}
$$

Recall from Equation 46 the principle of effective stress. As shown in Equation 49, this can extended to three-dimensions.

$$
\widetilde{\sigma}_{i j}=\frac{\sigma_{i j}}{1-d}
$$

Thus the law of elasticity coupled with the damage of the material is:

$$
\sigma_{i j}=\rho \frac{\partial \psi}{\partial \varepsilon_{i j}}=a_{i j k l} \varepsilon_{k l}^{e}(1-d)
$$

with $a$ as the fourth order elasticity stiffness tensor. The overall expression for the free energy in Equation 50 was defined by Lemaitre (1992) to be:

$$
\psi=\frac{1}{\rho}\left[\frac{1}{2} a_{i j k l} \varepsilon_{i j}^{e} \varepsilon_{k l}^{e}(1-d)+R_{\infty}\left[r+\frac{1}{b} \exp (-b r)\right]+\frac{X_{\infty} \gamma}{3} \alpha_{i j} \alpha_{i j}\right]
$$

where the values of $R$ and $b$ are related to the isotropic strain hardening of the material. Also, $X_{\infty}$ and $\gamma$ are parameters characterizing the non-linear kinematic strain hardening of the material. Therefore, the associated damage variable is found by Equation 52 .

$$
\bar{Y}=\rho \frac{\partial \psi}{\partial d}=-\frac{1}{2} a_{i j k l} \varepsilon_{i j}^{e} \varepsilon_{k l}^{e}
$$

It is now necessary to find a relationship between the damage variable and the elastic strain energy of the system. To simplify the development of this relation we define the value $(Y=-\bar{Y})$. By definition the increment of elastic strain energy density is given by Equation 53.

$$
d E_{e}=\sigma_{i j} d \varepsilon_{i j}^{e}
$$

Integrating this with the law of elasticity and assuming no variation of damage will give: 


$$
E_{e}=\int a_{i j k l} \varepsilon_{k l}^{e}(1-d) d \varepsilon_{i j}^{e}=\frac{1}{2} a_{i j k l} \varepsilon_{i j}^{e} \varepsilon_{k l}^{e}(1-d)
$$

and it follows that the value of the damage variable will be given by Equation 55 .

$$
Y=\frac{E_{e}}{1-d}
$$

The damage variable can also be written as one-half the variation of the strain energy density corresponding to the variation of damage (Chaboche, 1976). Beginning with the law of elasticity with constant stress:

$$
\delta \sigma_{i j}=a_{i j k l}\left[(1-d) \delta \varepsilon_{k l}^{e}-\varepsilon_{k l}^{e} \delta d\right]=0
$$

and

$$
\delta \varepsilon_{k l}^{e}=\varepsilon_{k l}^{e} \frac{\delta d}{1-d}
$$

with the definition for strain energy density from above

$$
\left.\delta E_{e}\right|_{\sigma=c o n s t}=\sigma_{i j} \delta \varepsilon_{i j}^{e}=\sigma_{i j} \varepsilon_{i j}^{e} \frac{\delta d}{1-d}=a_{i j k l} \varepsilon_{i j}^{e}(1-d) \varepsilon_{k l}^{e} \frac{\delta d}{1-d}
$$

or

$$
\left.\frac{\delta E_{e}}{\delta d}\right|_{\sigma=c o n s t}=a_{i j k l} \varepsilon_{i j}^{e} \varepsilon_{k l}^{e}
$$

thus

$$
Y=\frac{1}{2} \frac{\delta E_{e}}{\delta d}
$$

The above relationship for the damage variable provides a link between the strain energy and damage. This relationship was used by Ladevèze and Le Dantec (1992) to describe a model for damage accumulation in an individual lamina. A version of this 
model was implemented previously by Hurez et al. (1993) as a stand-alone FEA code to model static tensile tests on GLARE-2.

Ladevèze and Le Dantec (1992) developed the relationship in Equation 61 for the damaged material strain energy, $E_{D}$.

$$
E_{D}=\frac{1}{2}\left[\begin{array}{l}
\frac{\sigma_{11}^{2}}{E_{11}^{0}}-2 \frac{v_{12}^{0}}{E_{11}^{0}} \sigma_{1} \sigma_{2}+\frac{\left\langle\sigma_{22}\right\rangle_{+}^{2}}{E_{22}^{0}\left(1-d^{\prime}\right)}+\frac{\left\langle\sigma_{22}\right\rangle_{-}^{2}}{E_{22}^{0}} \\
+\frac{\sigma_{12}^{2}}{G_{12}^{0}(1-d)}+\frac{\sigma_{13}^{2}}{G_{12}^{0}(1-d)}+\frac{\sigma_{23}^{2}}{G_{23}^{0}}
\end{array}\right]
$$

where the subscripts 1,2 and 3 denote the fibre direction, the transverse direction and the through-thickness direction, respectively. The angled brackets and the \pm subscripts indicate negative or positive conditions for $\sigma_{11}$; therefore, if $\sigma_{22}$ is positive then $\left\langle\sigma_{22}\right\rangle_{-}^{2}$ is equal to zero. This allows different compressive and tensile conditions to be modelled. When $\sigma_{22}$ is compressive (negative) the matrix cracks close and do not affect the modulus. $E_{11}$ is the modulus in the fibre direction and $E_{22}$ is the modulus in the transverse direction. The two damage variables $\left(d\right.$ and $\left.d^{\prime}\right)$ act upon the initial transverse modulus and the shear modulus, $\left(E_{22}^{0}\right.$ and $\left.G_{12}^{0}\right)$ as shown in Equation 62.

$$
G_{12}=G_{12}^{0}(1-d) \quad \text { and } \quad E_{22}=E_{22}^{0}\left(1-d^{\prime}\right)
$$

Furthermore, two dissipation variables are associated with the damage terms $d$ and $d$ :

$$
\begin{gathered}
Y_{d^{\prime}}=\left.\rho \frac{\partial \psi}{\partial d}\right|_{\bar{\sigma}, d^{\prime}}=\left.\frac{\partial E_{D}}{\partial d}\right|_{\bar{\sigma}, d^{\prime}}=\frac{1}{2} \frac{\sigma_{12}^{2}}{G_{12}^{0}(1-d)^{2}} \\
Y_{d}=\left.\rho \frac{\partial \psi}{\partial d}\right|_{\bar{\sigma}, d}=\left.\frac{\partial E_{D}}{\partial d}\right|_{\bar{\sigma}, d}=\frac{1}{2} \frac{\left\langle\sigma_{22}\right\rangle_{+}^{2}}{E_{22}^{0}\left(1-d^{\prime}\right)^{2}}
\end{gathered}
$$

where $\psi$ is the free energy density and $\bar{\sigma}$ is the effective stress as given in Equation 64 . 


$$
\bar{\sigma}=\left[\begin{array}{l}
\sigma_{11} \\
\frac{\left\langle\sigma_{22}\right\rangle}{1-d^{\prime}}+\left\langle\sigma_{22}\right\rangle_{-} \\
\frac{\sqrt{2} \sigma_{12}}{(1-d)}
\end{array}\right]
$$

Two mechanisms were identified that contribute to the development of damage: matrix micro-cracking and fibre/matrix de-bonding. The following two quantities were introduced to describe the development of the damage:

$$
\begin{aligned}
& \underline{Y}=\left(\sqrt{Y_{d}+b Y_{d^{\prime}}}\right) \\
& \underline{Y^{\prime}}=\left(\sqrt{Y_{d^{\prime}}}\right)
\end{aligned}
$$

The damage development laws developed by Ladevèze and Le Dantec (1992) follow in Equations 66 and 67:

$$
d=\frac{\left\langle\underline{Y}-Y_{0}\right\rangle_{+}}{Y_{c}}
$$

if $d<1$ and $\underline{Y}^{\prime}<Y_{S}^{\prime}$; otherwise $d=1$ and the element fails. As well:

$$
d^{\prime}=\frac{\left\langle\underline{Y}-Y_{0}^{\prime}\right\rangle_{+}}{Y_{C}^{\prime}}
$$

if $d^{\prime}<1$ and $\underline{Y}<Y_{S}^{\prime}$; otherwise $d^{\prime}=1$ and the element fails.

The parameters $Y_{c}, Y_{c}^{\prime}, Y_{0}, Y_{0}^{\prime}$ and $b$ are characteristics of the material and are determined by tension tests as well as a few loading-unloading cycles on basic glassreinforced lamina as described by Ladevèze and Le Dantec (1992). The value $Y_{S}^{\prime}$ is the brittle-damage threshold that determines the behaviour of the fibre-matrix interface in the element transverse direction. 
Property evaluation tests are listed below along with some of the parameters that they provide. It should be noted that the testing also produces additional parameters used for plasticity modelling and compressive stiffness loss, two factors not used in the present project.

1. Tension tests of $\left[0^{\circ} / 90^{\circ}\right]_{2 \mathrm{~S}}$ or $\left[0^{\circ}\right]_{8}-E_{11}^{o}, v_{12}$, rupture stress and rupture strain

2. Tension tests of $\left[45^{\circ}\right]_{8}-E_{22}^{o}, G_{12}^{o}, Y_{c}$ and $Y_{o}$

3. Tension tests of $\left[ \pm 45^{\circ}\right]_{2 s}-G_{12}^{o}$ and $Y_{c}^{\prime}$

4. Tension tests of $[ \pm 67.5]_{2 \mathrm{~s}}-b$

As presented previously in Section 3.1.1, Hurez et al. (1993) applied a version of this model to simulate GLARE-2-2/1 under static tensile loading. Using the data from these tests Hurez et al. constructed an elementary CDM-based model for GLARE-2 under tension. The results agreed well with experiments for static loading and also for threepoint bending simulations. The Hurez et al. version was used to model a very specific situation - tensile loading of GLARE-2-2/1. This was a very limited application which was not useful for a dynamic event such as low-velocity impact. Therefore, a version of the Ladevèze and Le Dantec approach will be developed and implemented in LS-DYNA as a user-defined material model. This model will be used to predict the behaviour of GLARE panels subjected to impact loads.

These CDM theories have also been extended to include the prediction of delamination damage (Allix et al., 1991, Allix and Ladevèze, 1992, Daudeville and Ladevèze, 1993, Daudeville et al., 1995, Allix and Ladevèze 1995 and Allix et al., 1998).

Another CDM-based approach that has also been employed is the MLT method (Matzenmiller et al., 1995). This has been implemented in LS-DYNA by other 
researchers (Williams and Vaziri, 1995 and van Hoof, 1999). The MLT method is applied to unidirectional laminates by examining damage modes in each axis separately. Similar to the Ladevèze and Le Dantec approach, the MLT model does not include the effects of delamination in the overall damage relations as it only deals with the individual lamina. Damage is assumed to take the form of cracks perpendicular or parallel to the fibre direction in the unidirectional lamina. Hashin's (1980) failure criteria for lamina are the basis for the failure surfaces that bound the elastic loading region. Damage variables based upon a Weibull-type function are used to modify the stiffness matrix of the lamina. This method was developed for unidirectional lamina in composite materials. As discussed by Williams and Vaziri (2001) there are several weaknesses in the model. For example, it is not possible to model a material that is elastic up to failure but then displays gradual strain-softening after the peak load.

Based on the above review the Ladevèze and Le Dantec method was selected over the MLT method since the input properties for the GLARE prepreg are readily available in literature. Additionally, the Ladevèze and Le Dantec method focuses on damage in the transverse directions such as matrix cracking. This is one of the main damage modes in GLARE panels subjected to impact below the puncture threshold. 


\subsection{Subroutine Development}

As described in the preceding review, a version of the Ladevèze and Le Dantec (1992) model was implemented by Hurez et al. (1993) for application to GLARE-2. This model was implemented as a stand-alone program and could not be extended to other geometries or materials. Therefore, it was decided to develop the Ladevèze and Le Dantec model as a user-defined material subroutine, called a UMAT, in LS-DYNA. It is evident from Equations 66 and 67 that:

$$
\underline{Y}=d Y_{c}+Y_{o}=d^{\prime} Y_{c}^{\prime}+Y_{o}^{\prime}
$$

It is then possible to isolate $d^{\prime}$ as shown in Equation 69.

$$
d^{\prime}=\frac{d Y_{c}+Y_{o}-Y_{o}^{\prime}}{Y_{c}^{\prime}}
$$

Substituting Equations 63, 65 and 69 into Equation 67 and manipulating gives:

$$
\left(d Y_{c}-Y_{o}\right)^{2}-\left[\frac{1}{2} \frac{\sigma_{12}^{2}+\sigma_{13}^{2}}{G_{12}(1-d)^{2}}+\frac{b}{2} \frac{\sigma_{22}^{2}}{\left(1-\frac{d Y_{c}+Y_{c}-Y_{o}^{\prime}}{Y_{c}^{\prime}}\right)^{2}}\right]=0
$$

To remain consistent with the Ladevèze method a check for $\underline{Y}-Y_{o}>0$ must be performed; if this condition is not met then $d=d^{\prime}=0$. Equation 70 has up to 6 roots that were identified using the symbolic solution capabilities of Mathcad. In Fortran, this equation must be solved numerically. The only valid value of $d$ is slightly negative when the stresses are zero. As the applied stresses increase, the value of this root increases. None of the other possible solutions exhibit the same behaviour and none of them 
increase with increasing applied load between 0 and 1 . Since the range of valid values for $d$ is known to be between 0 and 1, it is best to use the Bisection Method rather than Newton's Method to find the root. The Bisection Method is also simple to implement in Fortran and converges quickly. Furthermore, when the stresses reach the rupture limit of the material the root becomes complex. At this point, it is not possible to find a root in the required range, therefore, the critical damage level has been reached. When this critical damage value is reached the element has failed.

The damage variables influence the stiffness of the orthotropic material. Thus, these terms are incorporated into the calculation of the stiffness matrix terms. Recall from Equation 38 that the incremental stresses in the orthotropic material are as follows:

$$
\Delta \sigma_{l}^{n+1 / 2}=\left[\begin{array}{c}
\sigma_{11} \\
\sigma_{22} \\
\sigma_{12} \\
\sigma_{23} \\
\sigma_{31}
\end{array}\right]=\left[\begin{array}{ccccc}
\frac{E_{11}}{1-v_{12} v_{21}} & \frac{v_{12} E_{22}}{1-v_{12} v_{21}} & 0 & 0 & 0 \\
\frac{v_{12} E_{22}}{1-v_{12} v_{21}} & \frac{E_{22}}{1-v_{12} v_{21}} & 0 & 0 & 0 \\
0 & 0 & G_{12} & 0 & 0 \\
0 & 0 & 0 & G_{23} & 0 \\
0 & 0 & 0 & 0 & G_{31}
\end{array}\right]\left[\begin{array}{c}
\dot{\varepsilon}_{11} \\
\dot{\varepsilon}_{22} \\
\dot{\varepsilon}_{12} \\
\dot{\varepsilon}_{23} \\
\dot{\varepsilon}_{31}
\end{array}\right]_{l}
$$

It should also be noted that the Poisson's ratios are calculated as follows (Hallquist, 1998):

$$
\begin{aligned}
& v_{21}=\frac{E_{22}}{E_{11}} v_{12} \\
& v_{13}=\frac{E_{33}}{E_{11}} v_{31} \\
& v_{32}=\frac{E_{33}}{E_{22}} v_{23}
\end{aligned}
$$

Since the damage variables $d$ and $d^{\prime}$ modify the transverse and shear stiffnesses of the material, it is simple to include them in the stress update. 


$$
\Delta \sigma_{l}^{n+1 / 2}=\left[\begin{array}{c}
\sigma_{11} \\
\sigma_{22} \\
\sigma_{12} \\
\sigma_{23} \\
\sigma_{31}
\end{array}\right]=\left[\begin{array}{ccccc}
b_{11} & b_{12} & 0 & 0 & 0 \\
b_{12} & b_{22} & 0 & 0 & 0 \\
0 & 0 & G_{12}(1-d) & 0 & 0 \\
0 & 0 & 0 & G_{23} & 0 \\
0 & 0 & 0 & 0 & G_{31}
\end{array}\right]\left[\begin{array}{c}
\dot{\varepsilon}_{11} \\
\dot{\varepsilon}_{22} \\
\dot{\varepsilon}_{12} \\
\dot{\varepsilon}_{23} \\
\dot{\varepsilon}_{31}
\end{array}\right]_{L}
$$

With the components as follows in Equations 74, 75 and 76.

$$
\begin{aligned}
& b_{11}=\frac{E_{11}}{1-v_{12}^{2} \frac{E_{22}\left(1-d^{\prime}\right)}{E_{11}}} \\
& b_{12}=\frac{v_{12} E_{22}}{1-v_{12}^{2} \frac{E_{22}\left(1-d^{\prime}\right)}{E_{11}}} \\
& b_{22}=\frac{E_{22}\left(1-d^{\prime}\right)}{1-v_{12} v_{12}^{2} \frac{E_{22}\left(1-d^{\prime}\right)}{E_{11}}}
\end{aligned}
$$

These components are then used to update the stresses as per Equation 73.

The basic damage model was first implemented in Mathcad to test the formulation and structure of the subroutine. The calculations were performed assuming only $\sigma_{12}$ loading for the F185/S2 glass prepreg in GLARE and the IM6/914 carbon prepreg tested by Ladevèze and Le Dantec (1992). This loading would occur in a prepreg layer oriented at $45^{\circ}$. The results of these validation calculations and the input parameters are shown in Table 20. Rupture occurs at a value of $d_{c}<1$ as anticipated by Kachanov. The experimental rupture stress of IM6/914 as measured by Ladevèze and Le Dantec (1992) is also provided. As can be seen in the table, the calculated rupture stress for the IM6/914 composite agrees very well with the experimental value reported by Ladevèze and Le Dantec (1992). Ladevèze and Le Dantec also quote a predicted value of $79 \mathrm{MPa}$. No 
data was available for the shear strength of the F185/S2 glass prepreg. However, Johnson (1975) gives $41 \mathrm{MPa}$ as the in-plane shear strength of a generic unidirectional, fibreglass/epoxy composite. This value is indicated with an asterix (*) in Table 20.

Table 20: Summary of shear rupture estimates and material properties for F185/S2 and IM6/914 prepregs.

\begin{tabular}{|c|c|c|c|}
\hline Property & Units & F185/S2 & IM6/914 \\
\hline$\left(\sigma_{12}^{r}\right)_{\text {prediction }}$ & $\mathrm{MPa}$ & 45 & 83 \\
\hline$\left(\sigma_{12}^{r}\right)_{\text {experiment }}$ & $\mathrm{MPa}$ & $41^{*}$ & 82 \\
\hline$d_{c}$ & & 0.470 & 0.473 \\
\hline$Y_{o}$ & $\mathrm{MPa}^{1 / 2}$ & 0.12 & 0.15 \\
\hline$Y_{o}^{\prime}$ & $\mathrm{MPa}^{1 / 2}$ & 0.0 & 0.24 \\
\hline$Y_{c}$ & $\mathrm{MPa}^{1 / 2}$ & 2.0 & 2.77 \\
\hline$Y_{c}{ }^{\prime}$ & $\mathrm{MPa}^{1 / 2}$ & 2.3 & 3.78 \\
\hline$b$ & $\mathrm{none}$ & 3.4 & 2.5 \\
\hline$Y_{s}$ & $\mathrm{MPa}$ & 1.1 & 0.7 \\
\hline$E_{11}$ & $\mathrm{GPa}$ & 53.98 & 170 \\
\hline$E_{22}$ & $\mathrm{GPa}$ & 9.412 & 10.8 \\
\hline$E_{33}$ & $\mathrm{GPa}$ & 9.412 & \\
\hline$G_{12}$ & $\mathrm{GPa}$ & 3.310 & 5.8 \\
\hline$v_{12}$ & none & 0.330 & 0.34 \\
\hline$v_{13}$ & none & 0.058 & \\
\hline$v_{23}$ & none & 0.058 & \\
\hline & & & \\
\hline
\end{tabular}

The main assumption with this material model is that damage forms due to the inplane shear and in-plane transverse stresses. The result of this assumption is that there is no stiffness loss in the fibre direction since no damage is predicted in this direction. Rupture in the fibre direction can be predicted using a strength limit with the assumption that the deformation is elastic up until rupture. The impact experiments and subsequent damage characterization described in Section 2.2 showed that there was no fibre damage below the puncture threshold in the impacted FMLs. Damage consisted of matrix cracks, transverse cracks and delamination. Therefore, fibre damage was not incorporated into the prepreg model. One method for including these effects is to use Hashin's criteria for fibre failure as described by Matzenmiller et al. (1995). Fibre damage could be 
incorporated provided the appropriate strength parameters were measured or available in literature. This would not be difficult, but was not required in the present application.

When the damage level predicted by the model in an element reaches the critical value the values of $d$ and $d^{\prime}$ are immediately set to 1 and the element fails. The element can also fail if the numerical limits in Equations 66 and 67 are exceeded. Two options are available for handling the failed element. The first is to use the built-in element deletion capability of LS-DYNA to completely remove the element from the mesh. If this approach is used, the element is not included in any further stress calculations and load carrying capacity in all directions is lost. However, this is not realistic; in fact, when compressive loads are applied, matrix cracks would close and load would continue to be transmitted. An additional problem with deleting the elements is that the aluminum layers that had been separated by the prepreg layer can then come into contact. This situation, of course, does not occur in the experiments. Even after delamination and cracking have occurred in the prepreg layers, they still carry loads and keep the aluminum layers separate. Furthermore, in LS-DYNA it is impractical to establish multiple contact definitions for layers that might come into contact if the elements between them fail.

Another method for handling the failed element is to set the stresses in the tensile 22 and shear 12 directions to zero. This is performed over 100 time steps to prevent a stress wave from the sudden loss of stiffness. Stresses in the tensile and compressive 11, 22 and 33 directions are still calculated. This allows the element to continue to carry compressive loads in all directions and tensile loads in the fibre direction. This technique was incorporated into the UMAT. 


\subsection{Validation of Elastic Model}

A pair of coupon simulations were conducted using the CDM-based user-defined subroutine with damage deactivated and the standard orthotropic-elastic (type 2) model. The purpose of these simulations was to validate the portions of the code not related to the calculation of damage in the prepreg material. The formulation of the elastic portions is identical to the definition of the orthotropic-elastic (type 2) material in LS-DYNA. The coupon stresses and strains predicted by the user-defined material model (UMAT) should be identical to values predicted using the orthotropic-elastic (type 2) material model. The coupon used for these simulations is shown in Figure 76.

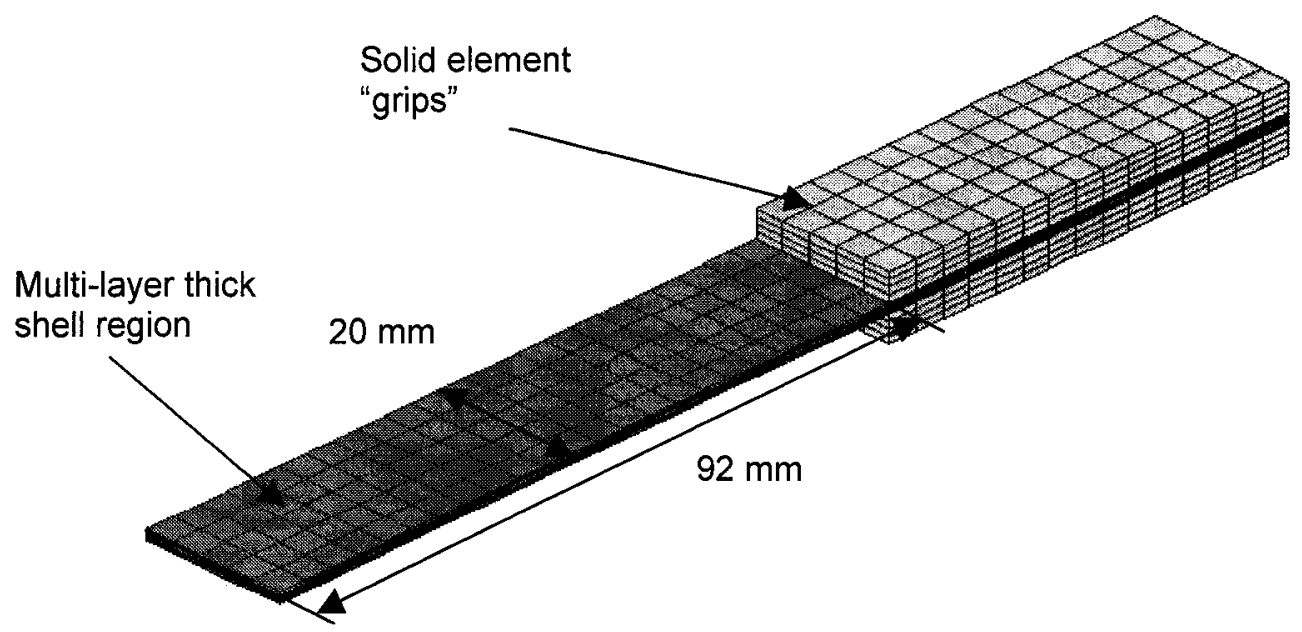

Figure 76: Prepreg elastic response verification tensile coupon (half-geometry).

The first simulation used the standard LS-DYNA orthotropic-elastic material model and the second used the UMAT with the damage calculation portions deactivated. The lay-up of the coupon was chosen as $\left[15^{\circ}\right]_{8}$. The elastic properties of the prepreg material are summarized in Table 21. The stress-strain response of the two simulations is shown in Figure 77. There is very good agreement between the two material models. 
Table 21: Elastic properties of the F185/S2 prepreg material.

\begin{tabular}{|c|c|c|}
\hline Property & Units & Value \\
\hline$E_{11}$ & $\mathrm{GPa}$ & 53.98 \\
\hline$E_{22}$ & $\mathrm{GPa}$ & 9.412 \\
\hline$E_{33}$ & $\mathrm{GPa}$ & 9.412 \\
\hline $\mathrm{G}_{12}$ & $\mathrm{GPa}$ & 3.310 \\
\hline$v_{12}$ & none & 0.330 \\
\hline$v_{13}$ & none & 0.058 \\
\hline$v_{23}$ & none & 0.058 \\
\hline
\end{tabular}

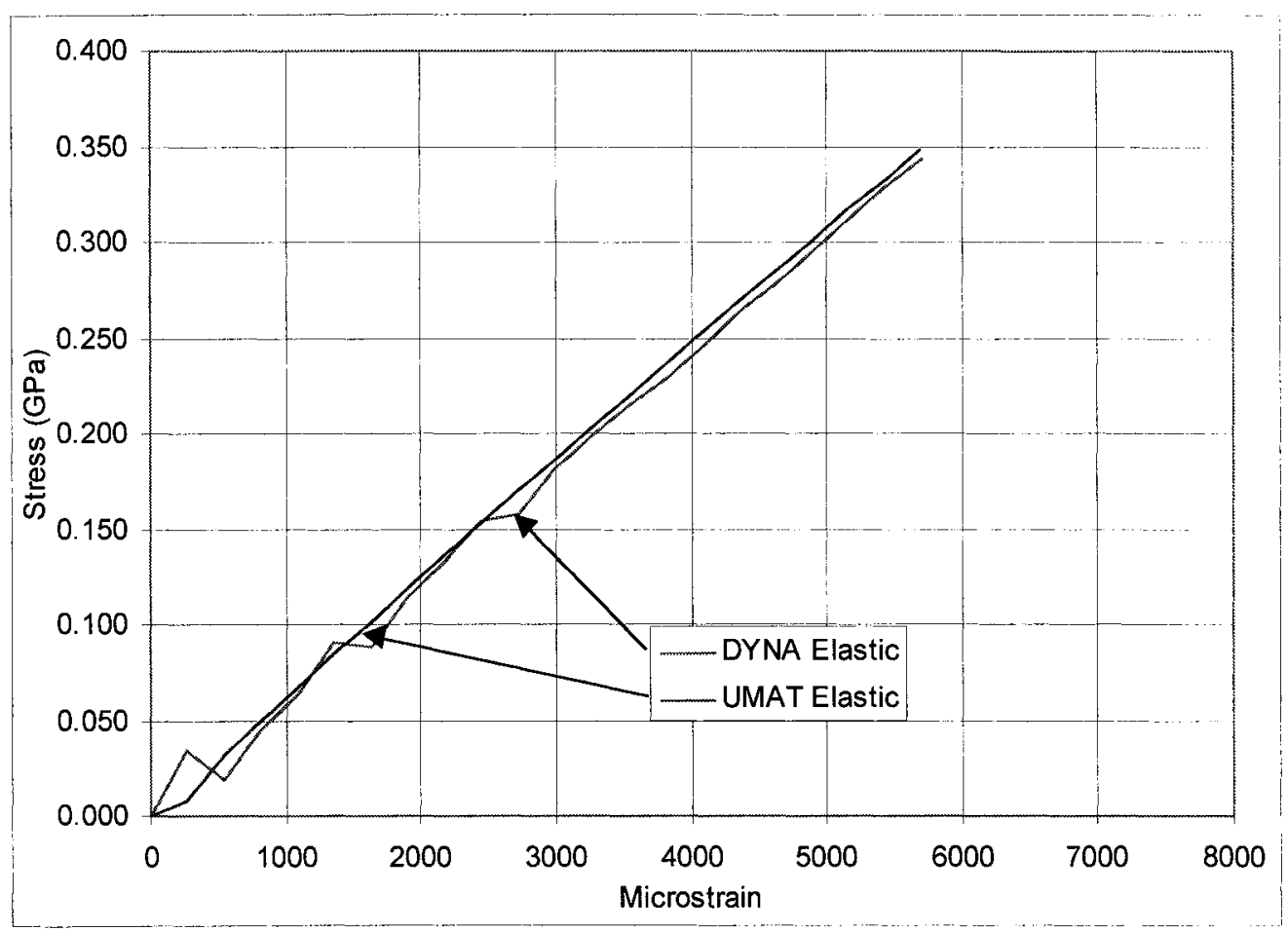

Figure 77: Comparison of simulated stress-strain response for prepreg coupon using the orthotropic-elastic model and the UMAT without damage. 


\subsection{Validation of the Damage Model}

This section describes the results of finite element simulations and experiments carried out on GLARE coupons. The purpose of these tests was to verify the UMAT prior to implementing it for impact simulations. A series of static tensile tests was conducted using various types of GLARE-3. These tests were conducted to provide data to support other ongoing tasks on the FML Project at IAR-SMPL. As will be described below, some of these data were used for the CDM-based model validation. Validating the damage portions of the CDM-based model prior to application to impact simulations was necessary to eliminate the possibility of problems with the model.

\subsubsection{Static Tensile Testing of GLARE}

Coupons of GLARE-3-3/2, GLARE-3-4/3 and GLARE-3-5/4 were subjected to static tensile loading to measure their tensile properties. The test methodology and specimen design were conducted in accordance with the FML tensile test method described by Mattousch (1993). Specimens were cut from large GLARE panels to the dimensions shown in Figure 78. 


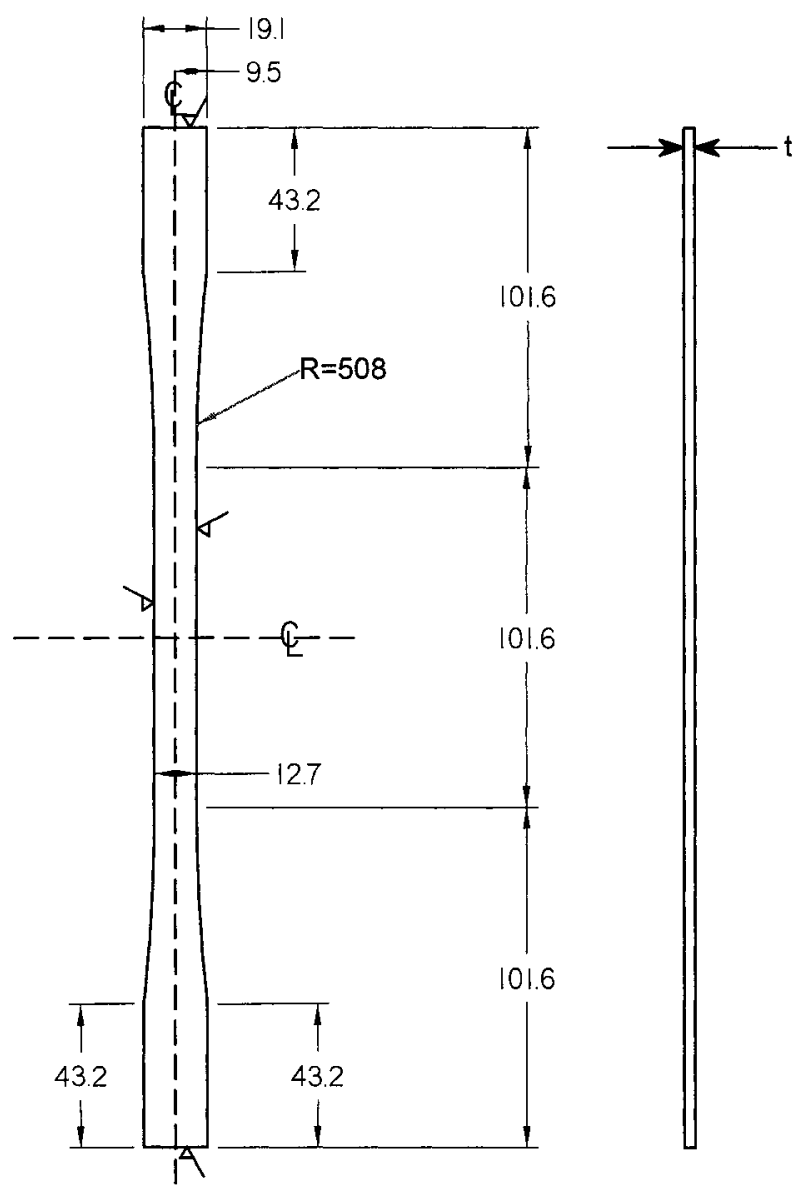

Figure 78: Static tensile strength coupon.

Specimens were tested in an Instron load frame at IAR-SMPL with a loading rate of $2 \mathrm{~mm} / \mathrm{min}$. Displacement data was collected using an extensometer attached to the centre of the specimen. Results from the tests are summarized in Table 22. These results are to be published in an IAR-SMPL laboratory memorandum at a future date.

Table 22: Summary of static tensile results for the GLARE-3 test coupons. Each data point is an average of 5 tests.

\begin{tabular}{|l|c|c|c|c|}
\hline \multirow{2}{*}{ Specimen ID } & Modulus & Yield Stress & \% Elongation & UTS \\
\cline { 5 - 5 } & $\mathbf{M P a}$ & $\mathbf{M P a}$ & at Failure & $\mathbf{M P a}$ \\
\hline $\mathrm{GL}-3-3 / 20^{\circ}$ & 55443 & 304 & 4.5 & 698 \\
\hline $\mathrm{GL}-3-3 / 215^{\circ}$ & 53198 & 270 & 6.0 & 521 \\
\hline $\mathrm{GL}-3-4 / 30^{\circ}$ & 56458 & 307 & 4.2 & 705 \\
\hline $\mathrm{GL}-3-4 / 315^{\circ}$ & 53417 & 269 & 5.9 & 526 \\
\hline $\mathrm{GL}-3-5 / 40^{\circ}$ & 51930 & 296 & 4.5 & 713 \\
\hline $\mathrm{GL}-3-5 / 415^{\circ}$ & 51534 & 261 & 6.4 & 532 \\
\hline
\end{tabular}


The GLARE-3-3/2 data were then used to validate the CDM-based damage model through comparisons with coupon simulations in LS-DYNA. Typical measured stressstrain curves for the $0^{\circ}$ and $15^{\circ}$ directions are shown in Figure 79.

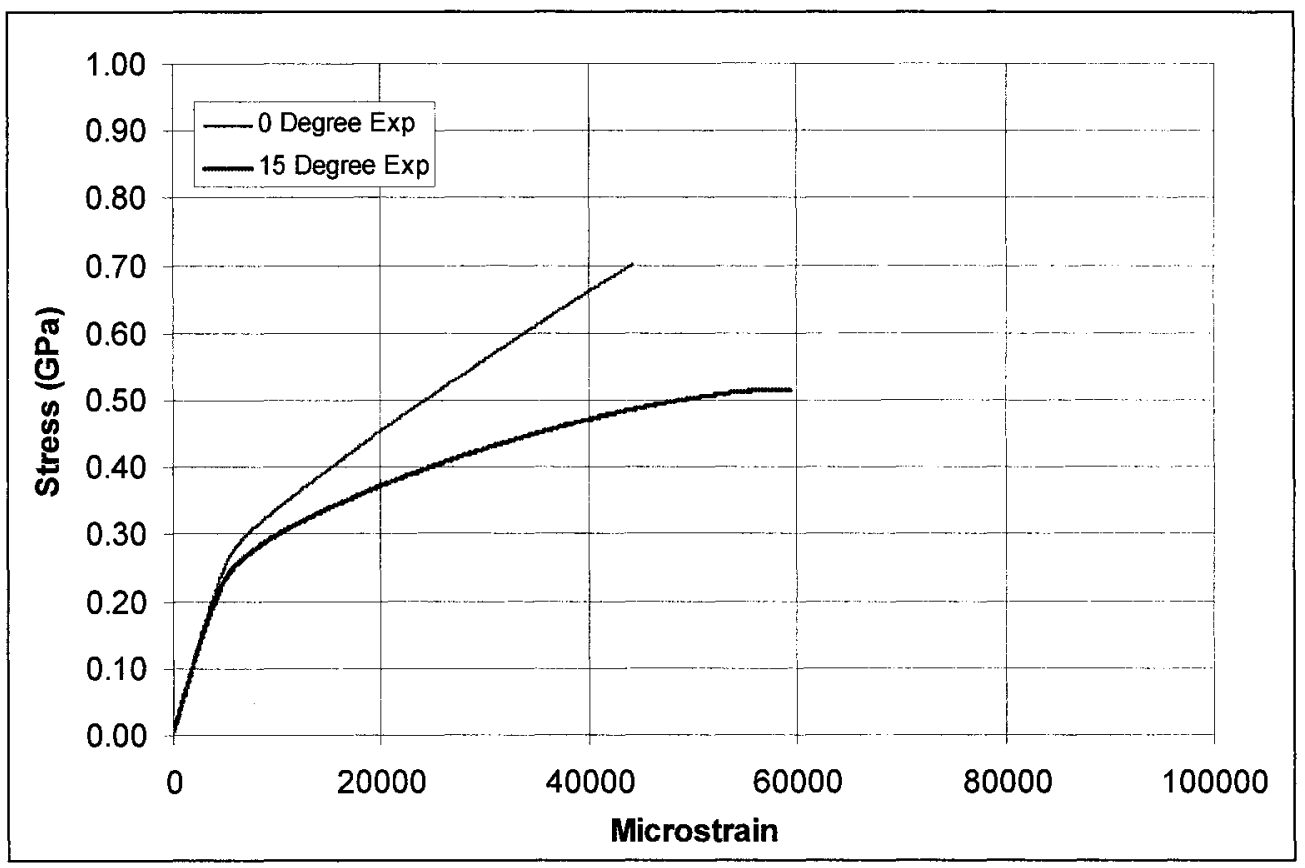

Figure 79: Typical GLARE-3-3/2 measured stress-strain curves for $0^{\circ}$ and $15^{\circ}$.

\subsubsection{Tensile Simulations of GLARE in LS-DYNA}

Simulations were developed to provide results for comparison to those obtained from the experiments described in Section 6.4.1. Two simulated geometries were prepared. The low-density mesh coupon geometry is shown in Figure 80. This coupon has straight edges and was suggested by Ladevèze and Le Dantec (1992) for validating the CDM theory. The high-density mesh coupon is shown in Figure 81. This coupon represented the experimental configuration shown in Figure 78. As can be seen in these two figures, the high-density coupon is 10 elements wide in the test section, whereas the low-density coupon is only 5 elements wide. Both simulated coupons consisted of a test 
region and "grips" that transfer the loads into the test region. Half-symmetry was used to reduce computational requirements. A complete list of the coupon simulations carried out is presented in Table 23 .

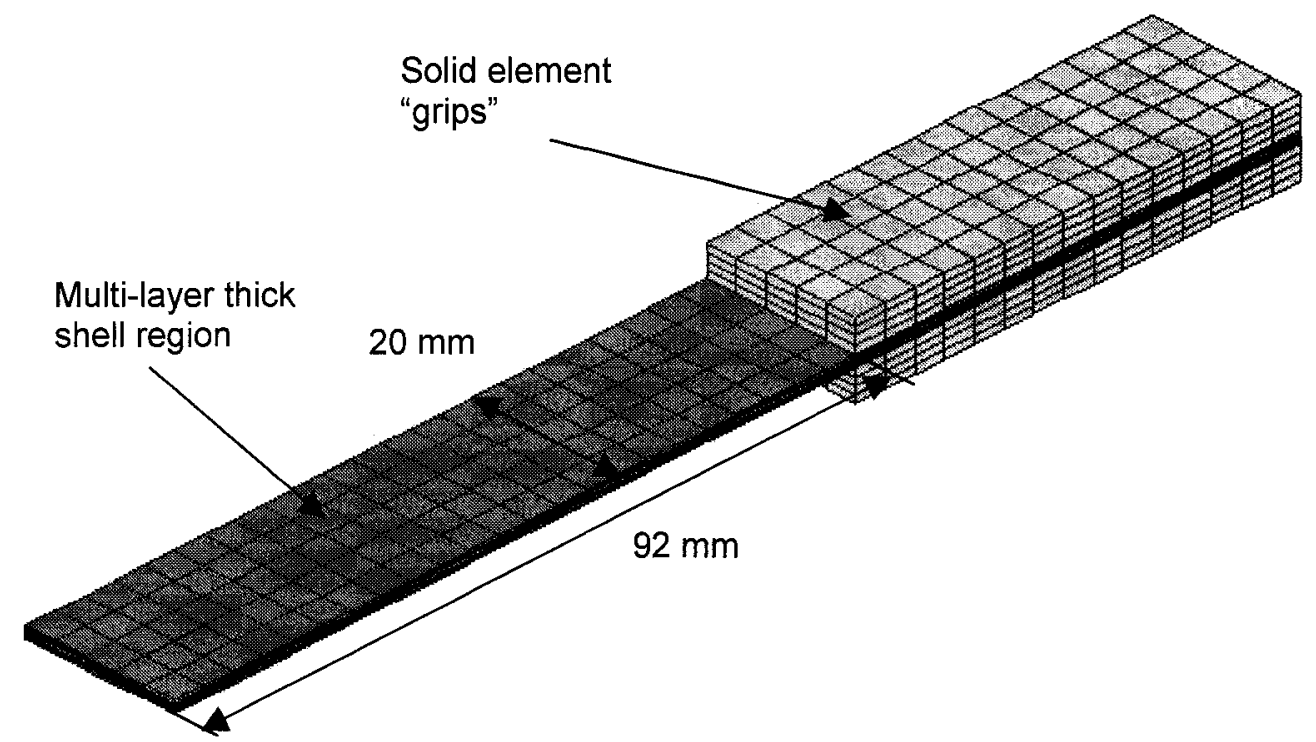

Figure 80: Low-density mesh GLARE coupon geometry.

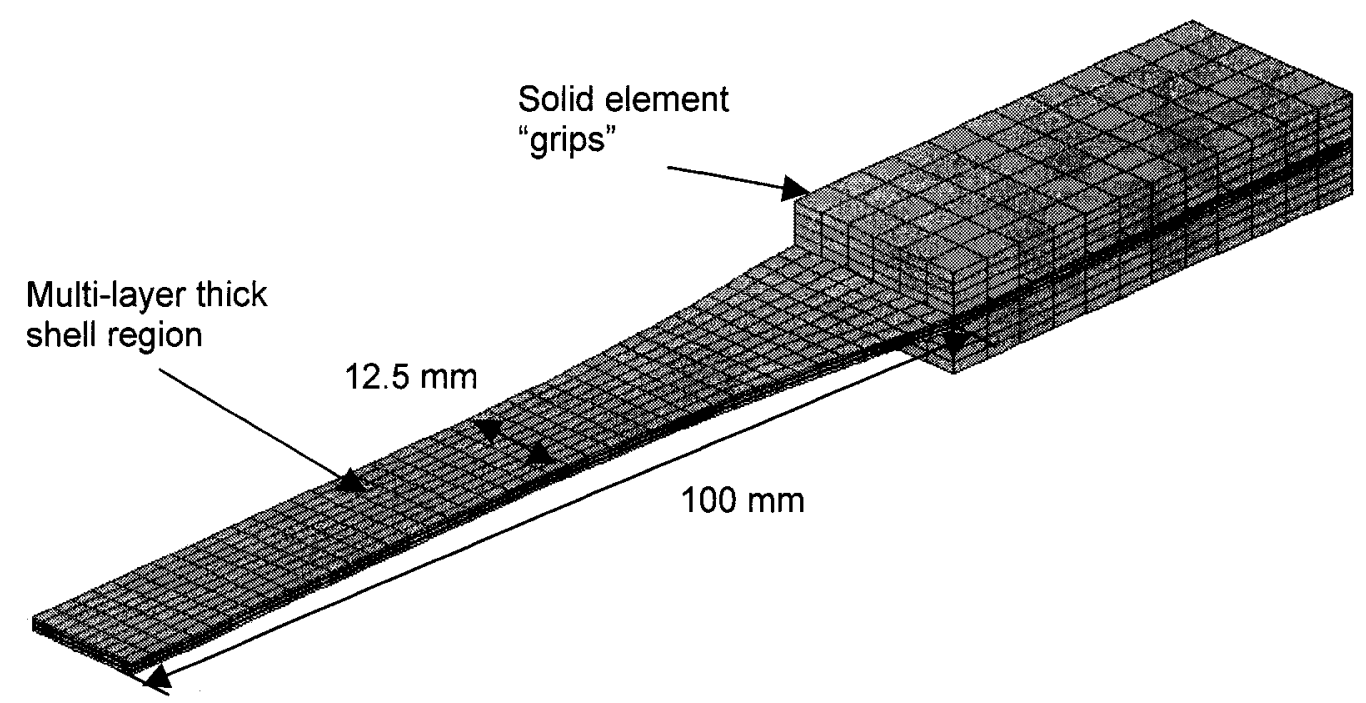

Figure 81: High-density mesh GLARE coupon geometry. 
Table 23: List of prepreg and GLARE-3-3/2 coupon simulations.

\begin{tabular}{|c|c|l|c|}
\hline SIM ID & Material Model & \multicolumn{1}{|c|}{ Lay-up } & $\begin{array}{c}\text { Mesh } \\
\text { Density }\end{array}$ \\
\hline 1 & Orthotropic-elastic & {$\left[2024 /\left(0^{\circ} / 90^{\circ}\right) / 2024 /\left(90^{\circ} / 0^{\circ}\right) / 2024\right]$} & Low \\
\hline 2 & Orthotropic-elastic & {$\left[2024 /\left(15^{\circ} / 105^{\circ}\right) / 2024 /\left(105^{\circ} / 15^{\circ}\right) / 2024\right]$} & Low \\
\hline 3 & UMAT & {$\left[2024 /\left(0^{\circ} / 90^{\circ}\right) / 2024 /\left(90^{\circ} / 0^{\circ}\right) / 2024\right]$} & Low \\
\hline 4 & UMAT & {$\left[2024 /\left(15^{\circ} / 105^{\circ}\right) / 2024 /\left(105^{\circ} / 15^{\circ}\right) / 2024\right]$} & Low \\
\hline 5 & UMAT & {$\left[2024 /\left(0^{\circ} / 90^{\circ}\right) / 2024 /\left(90^{\circ} / 0^{\circ}\right) / 2024\right]$} & High \\
\hline 6 & UMAT & {$\left[2024 /\left(15^{\circ} / 105^{\circ}\right) / 2024 /\left(105^{\circ} / 15^{\circ}\right) / 2024\right]$} & High \\
\hline
\end{tabular}

These simulations used prepreg properties published by Hurez et al. (1993) as listed in Table 24. The aluminum properties were presented earlier in Section 4.1. The aluminum layers were $0.3 \mathrm{~mm}$ thick and the prepreg layers were $0.125 \mathrm{~mm}$ thick. The coupons were composed of 7 layers of thick-shell elements.

Table 24: Summary of prepreg constituent properties.

\begin{tabular}{|c|c|c|}
\hline Property & Units & Value \\
\hline \hline$Y_{o}$ & $\mathrm{MPa}^{1 / 2}$ & 0.12 \\
\hline$Y_{o}^{\prime}$ & $\mathrm{MPa}^{1 / 2}$ & 0.00 \\
\hline$Y_{c}$ & $\mathrm{MPa}^{1 / 2}$ & 2.00 \\
\hline$Y_{c}^{\prime}$ & $\mathrm{MPa}^{1 / 2}$ & 2.30 \\
\hline$b$ & $\mathrm{none}$ & 3.40 \\
\hline$Y_{s}$ & $\mathrm{MPa}$ & 1.10 \\
\hline$E_{11}$ & $\mathrm{GPa}$ & 53.98 \\
\hline$E_{22}$ & $\mathrm{GPa}$ & 9.412 \\
\hline$E_{33}$ & $\mathrm{GPa}$ & 9.412 \\
\hline$G_{12}$ & $\mathrm{GPa}$ & 3.310 \\
\hline$v_{12}$ & none & 0.330 \\
\hline$v_{13}$ & none & 0.058 \\
\hline$v_{23}$ & none & 0.058 \\
\hline
\end{tabular}

Thick-shell elements were used to represent the GLARE coupons and solid-bricks were used for the "grip" load introduction components. No delamination was modelled in these simulations. The orthotropic-elastic simulations (1 and 2) modelled the prepreg material as elastic without damage. The UMAT (3-6) simulations used the CDM-based prepreg model. In all cases the aluminum was modelled using the elastic-plastic model 
described previously. The simulations were not carried to complete rupture of the specimens since the material model did not incorporate fibre fracture.

The two coupons were selected to study the effect of mesh density on the results. The effect of this increase in mesh density on the stress-strain response is shown in Figure 82. Two pairs of stress-strain curves are shown in this figure. The first pair labelled " 3 " and " 5 " have the material oriented at $0^{\circ}$ with respect to the loading direction. Simulation 3 uses the low-density coupon and simulation 5 uses the high-density coupon. The second pair of simulations had the material oriented at $15^{\circ}$ with respect to the loading direction. Simulation 4 used the low-density coupon and simulation 6 used the highdensity coupon.

For the lower density mesh used in simulation 4 , there are drops in the stressstrain curve near the end of the simulation. These occur when one of the elements fails and its load carrying capacity is eliminated. Since there are only a few elements across the width of the specimen, a significant portion of the strength of the coupon is lost suddenly. The higher density mesh used in simulations 5 and 6 did not show the same effect and the stress-strain curve is somewhat smoother. The stress drops were not observed in the $0^{\circ}$ simulations for either mesh density. 


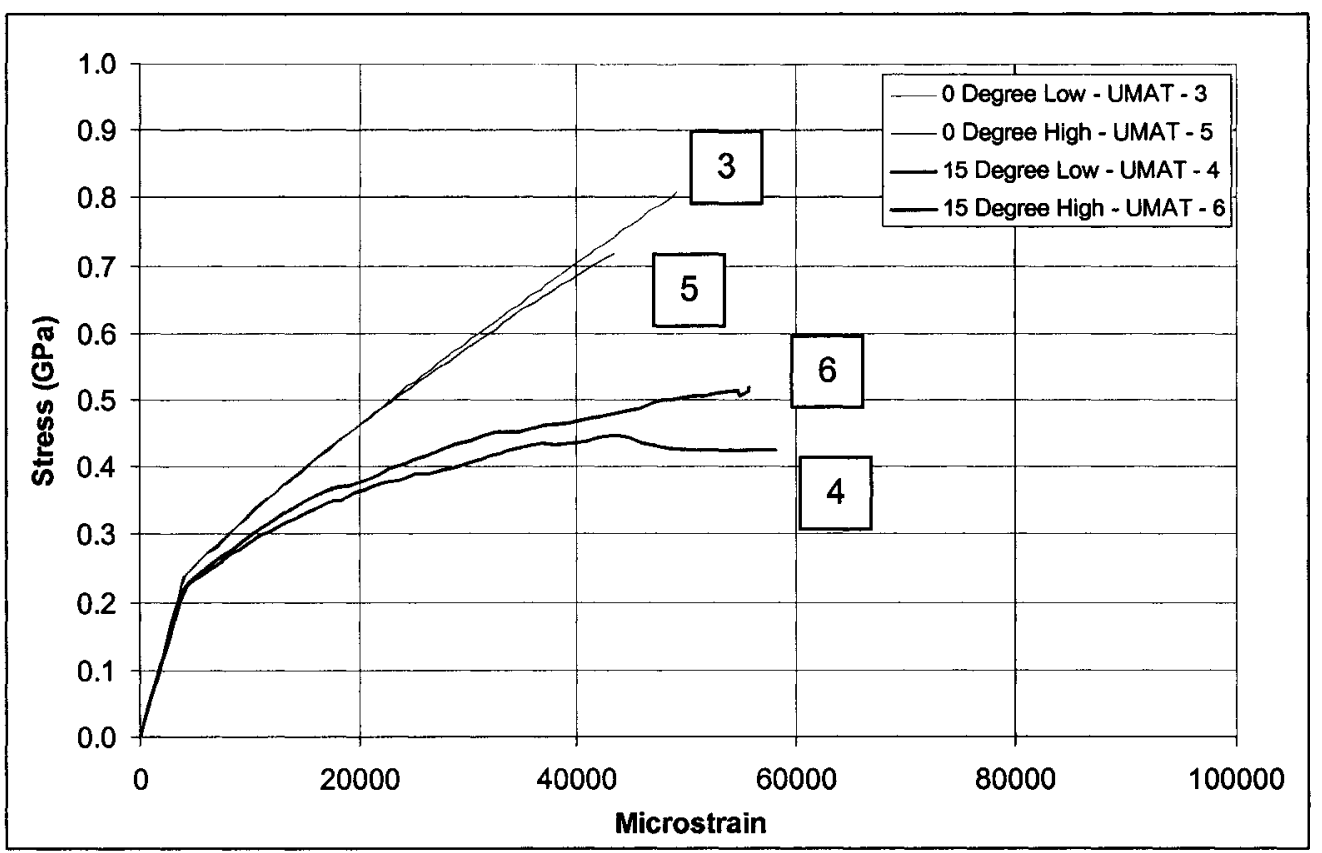

Figure 82: Effect of mesh density on the stress-strain response of the GLARE coupons.

As shown in Figure 83, there was good agreement between the experimental stress-strain measurements and the simulated results for GLARE-3-3/2 in the $0^{\circ}$ direction. The graph compares the results of the experiment to simulations using the low- (3) and high- (5) density meshes with the UMAT, and low-density mesh using the UMAT without damage for the prepreg (1). As would be expected, the orthotropic-elastic simulation (1) was stiffer than the experiment for the $0^{\circ}$ case. The orthotropic-elastic model does not account for any stiffness degradation in the prepreg.

For the $0^{\circ}$ cases ( 3 and 5 ) with damage, the results show slightly reduced stiffness compared to the $0^{\circ}$ orthotropic-elastic simulation (1). In the $0^{\circ}$ fibre-direction, the UMAT calculates no stiffness losses and only stiffness losses in the $90^{\circ}$ fibre layers are calculated. Again, in practice there would be some damage formed by fibre fracture and also matrix deformation in the $0^{\circ}$ plies, that is not accounted for in the model. 


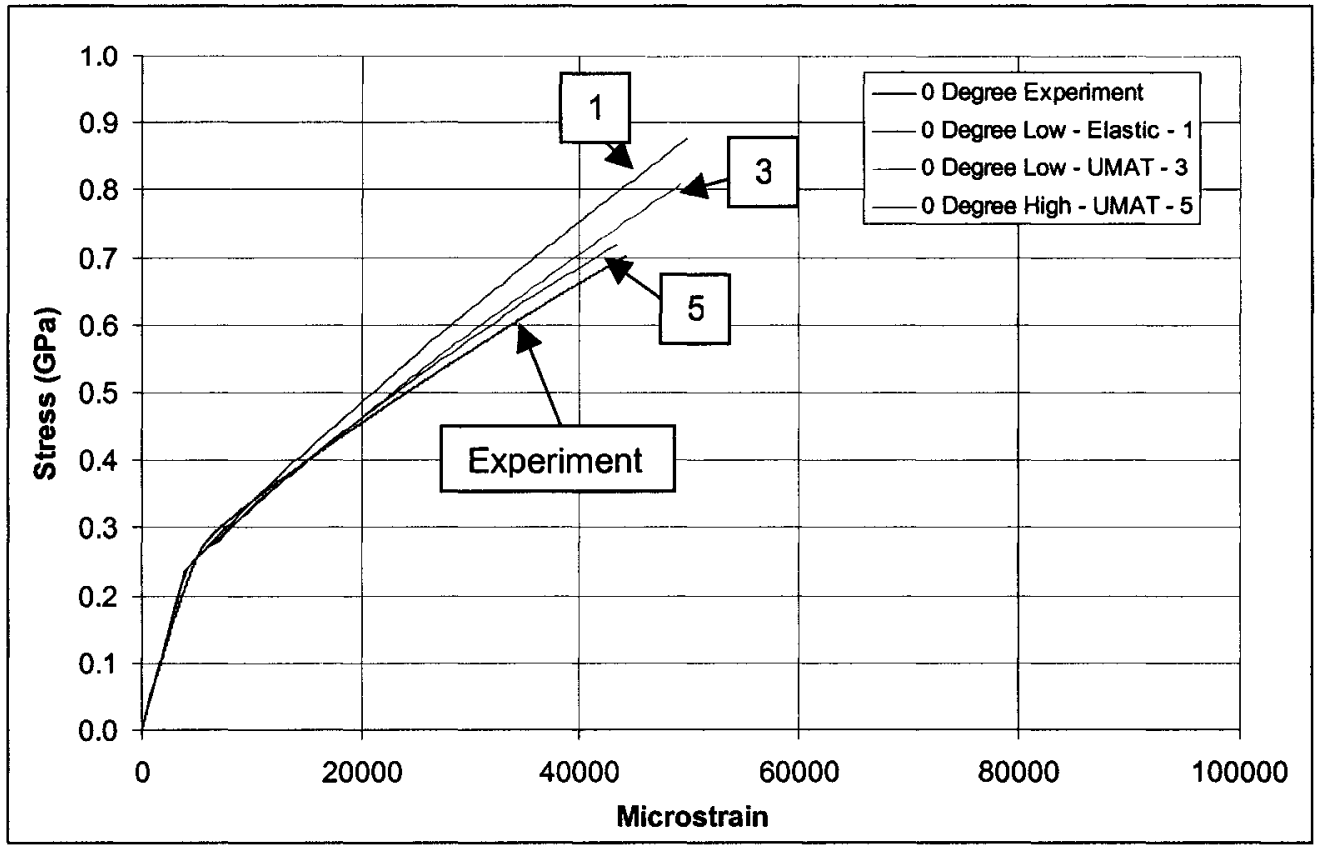

Figure 83: Comparison between simulated stress-strain response and experimental data for $0^{\circ}$ GLARE-3-3/2.

For the off-axis $15^{\circ}$ cases ( 4 and 6 ) the results obtained with the UMAT model are closer to the experimental results as shown in Figure 84. However, there is more strength loss predicted by the UMAT model for simulation 4 than was observed in the experiments. This is the effect of the lower mesh density as described above. The simulation results for the high-density mesh (6) show very good agreement with the experiments. 


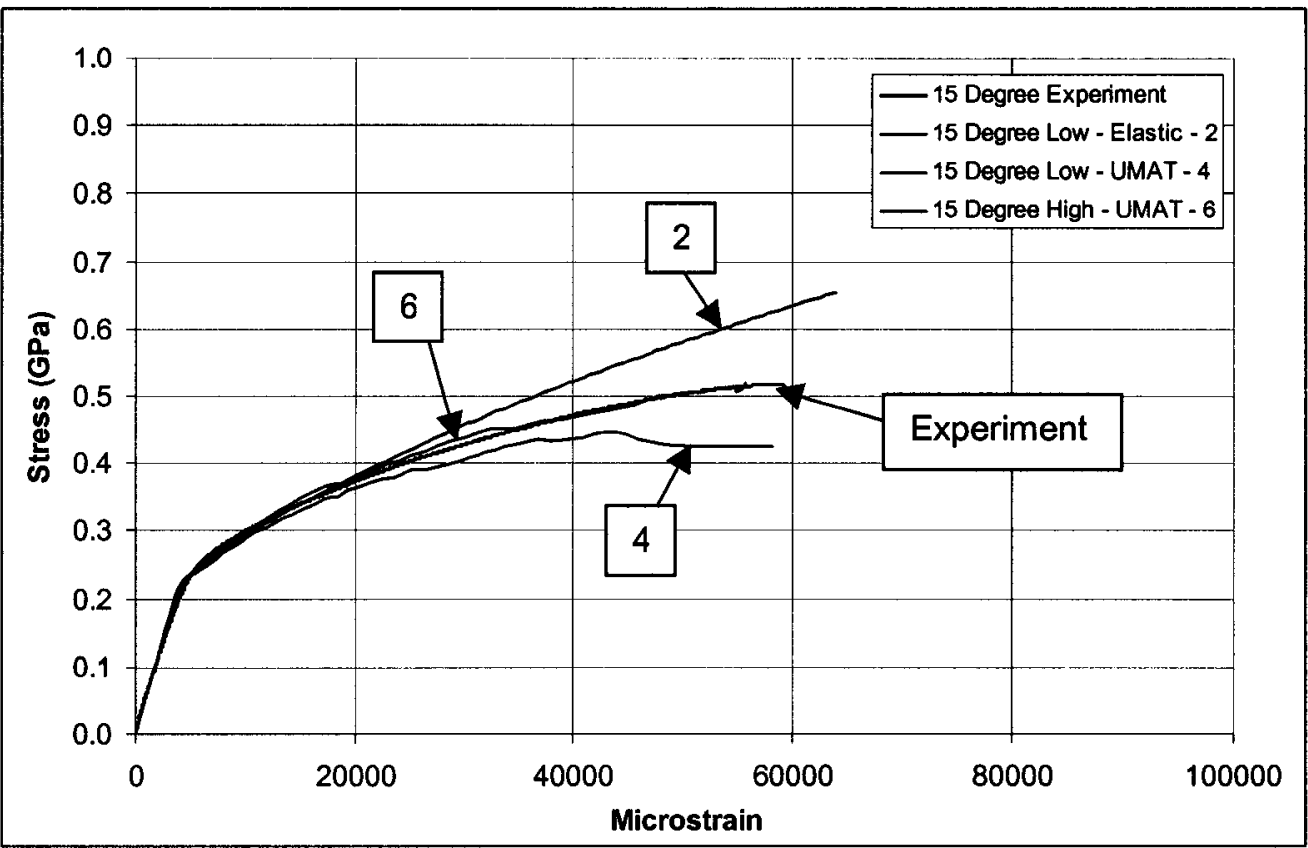

Figure 84: Comparison between simulated response and experimental data for $1^{\circ}$ GLARE-3-3/2 loaded in tension.

The GLARE coupon simulations successfully demonstrated the application of a CDM-based model to predict the tensile response of GLARE. The UMAT was then applied to model the behaviour of the GLARE prepreg material in complete impact simulations. 


\section{CHAPTER 7}

\section{SIMULATION OF LOW-}

\section{VELOCITY IMPACTS ON}

\section{GLARE LAMINATES}

Based upon the success of the UMAT simulations in predicting the tensile response of GLARE, the modelling methodology was applied to low-velocity impact simulations. These simulations duplicate the experiments presented in Chapter 2. The effects of several parameters were studied including mesh density, interface type and the type of material model. Section 7.1 describes the application of the damage modelling methodology and how the individual components from Chapters 4,5 and 6 were combined. Sections 7.2, 7.3 and 7.4 discuss details of the input parameters, mesh sensitivity and simulation results. 


\subsection{Application of the FML Damage Modelling Methodology}

The components of the FML damage modelling methodology presented in Chapters 4, 5 and 6 were assembled to model the impact response of GLARE coupons. The most important part of the methodology is the CDM-based prepreg model. The properties described by Ladevèze and Le Dantec (1992) and Hurez et al. (1993) were used in this material model. The material model was implemented in LS-DYNA as described in Section 6.2. The normal and shear strength properties of the interface were provided in the manufacturer's data sheets for the adhesive. It was not possible to employ the data presented in Section 5.2.4 to model the post-delamination response. Therefore, the correction applied to the interface was a linear stress-displacement curve. The aluminum layers were modelled using the elastic-plastic (type 24) material model in LS-DYNA with material properties as presented in Section 4.1.

The components of the modelling methodology were applied as follows:

1. Elastic-plastic aluminum, orthotropic elastic prepreg and tied-interface,

2. Elastic-plastic aluminum, orthotropic elastic prepreg and uncorrected tiebreakinterface,

3. Elastic-plastic aluminum, orthotropic elastic prepreg and corrected-tiebreak interface, and

4. Elastic-plastic aluminum, UMAT prepreg and simple-tiebreak interface. 


\subsection{GLARE Simulation Input Parameters}

The geometry of the simulations was identical to that used for the final aluminum simulations described in Chapter 4. For reference, the mesh geometry is repeated in Figure 85. The aluminum layers were modelled with the elastic-plastic (type 24) material model using properties presented previously in Chapter 3 and repeated for reference in Table 25 and Figure 86. The prepreg layers were modelled using either the orthotropicelastic (type 2) material (Section 3.3.6) or the UMAT (Chapter 6). For reference, the prepreg properties are repeated in Table 26. All of the layers of the simulations were modelled using thick-shell elements.

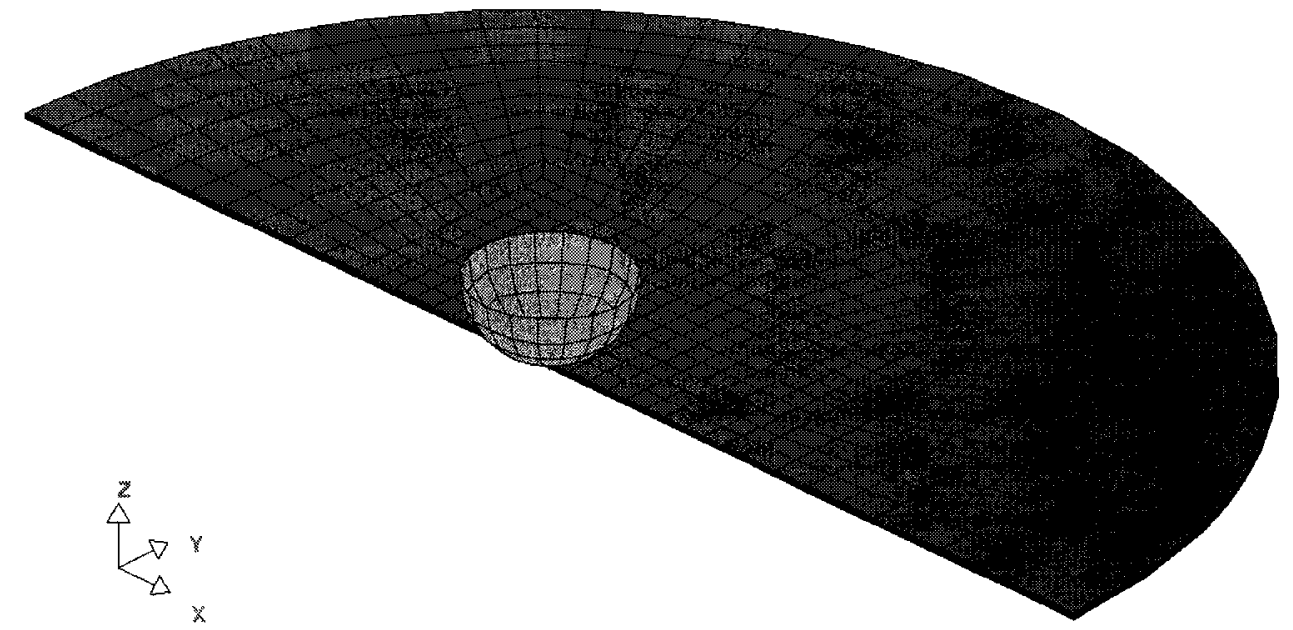

Figure 85: Mesh geometry for GLARE impact simulations.

Table 25: Aluminum material properties.

\begin{tabular}{|c|c|c|}
\hline Property & Value & Unit \\
\hline \hline Young's Modulus & 72.39 & $\mathrm{GPa}$ \\
\hline Yield Stress & 285 & $\mathrm{MPa}$ \\
\hline Mass Density & $2.643 \times 10^{-6}$ & $\mathrm{Kg} / \mathrm{mm}^{3}$ \\
\hline Poisson's Ratio & 0.33 & - \\
\hline
\end{tabular}




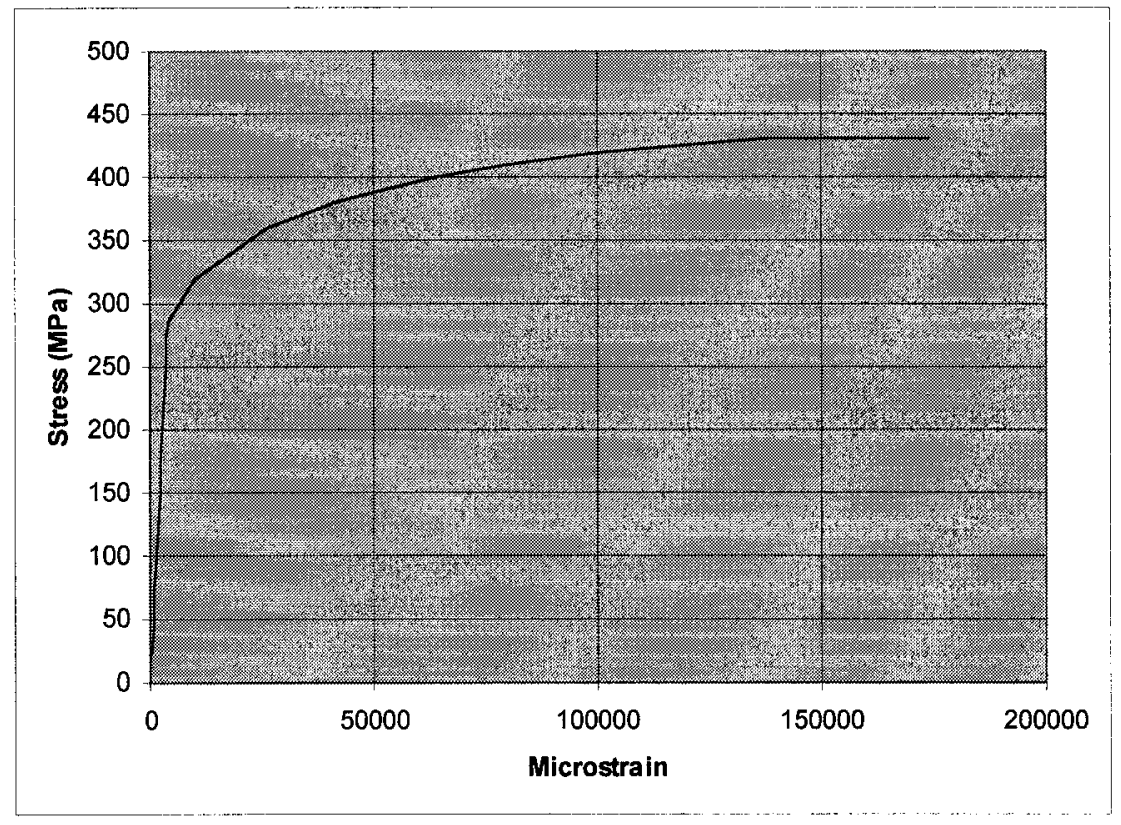

Figure 86: Stress-strain curve for aluminum 2024-T3.

Table 26: Prepreg material input properties.

\begin{tabular}{|c|c|c|}
\hline Property & Units & Value \\
\hline$Y_{0}$ & $\mathrm{MPa}^{1 / 2}$ & 0.12 \\
\hline$Y_{0}^{\prime}$ & $\mathrm{MPa}^{1 / 2}$ & 0.00 \\
\hline$Y_{c}$ & $\mathrm{MPa}^{1 / 2}$ & 2.00 \\
\hline$Y_{c}^{\prime}$ & $\mathrm{MPa}^{1 / 2}$ & 2.30 \\
\hline$b$ & none & 3.40 \\
\hline$Y_{s}$ & $\mathrm{MPa}^{1 / 2}$ & 1.10 \\
\hline$Y_{r}$ & $\mathrm{MPa}$ & 2.00 \\
\hline$E_{11}$ & $\mathrm{GPa}$ & 53.98 \\
\hline$E_{22}$ & $\mathrm{GPa}$ & 9.412 \\
\hline$E_{33}$ & $\mathrm{GPa}$ & 9.412 \\
\hline$G_{12}$ & $\mathrm{GPa}$ & 3.310 \\
\hline$V_{12}$ & none & 0.330 \\
\hline$V_{13}$ & none & 0.058 \\
\hline$V_{23}$ & none & 0.058 \\
\hline
\end{tabular}

Simulations were carried out with three different interface modelling techniques. These models were discussed in detail in Section 3.3.5. To review, the tied-interface connects the layers together without delamination. Next, the simple-tiebreak interface uses the tiebreak model with the manufacturer's shear and normal interface strengths of $35 \mathrm{MPa}$ and $48 \mathrm{MPa}$. Finally, the corrected-tiebreak interface method uses the tiebreak 
model with a stress-displacement correction curve to model the post-failure behaviour. This curve defines the delamination opening resistance stress as a function of the separation distance between the nodes at the contact interface. The simplest model is a linear decrease in opening stress as the separation distance increases. The curve starts at the normal strength of the adhesive and decreases linearly to zero over a distance of 1.5 $\mathrm{mm}$. This distance was chosen based on the destructive inspections presented in Chapter 2. The scale of the delamination openings was on the order of 1 to $2 \mathrm{~mm}$. The assumed stress-displacement curve is shown in Figure 87.

It was found, for both the simple- and corrected-tiebreak interface simulations with elastic prepreg, that the manufacturer's properties were too high to allow interface failure. The values were lowered until delamination occurred in the specimens. This occurred with property values at approximately 0.025 of the quoted strengths. As described in Section 5.2.4, the application of the DCB data to correct this response was unsuccessful. When the UMAT was used with the simple-tiebreak interface, the unchanged manufacturer's properties were successfully employed to predict delamination. 


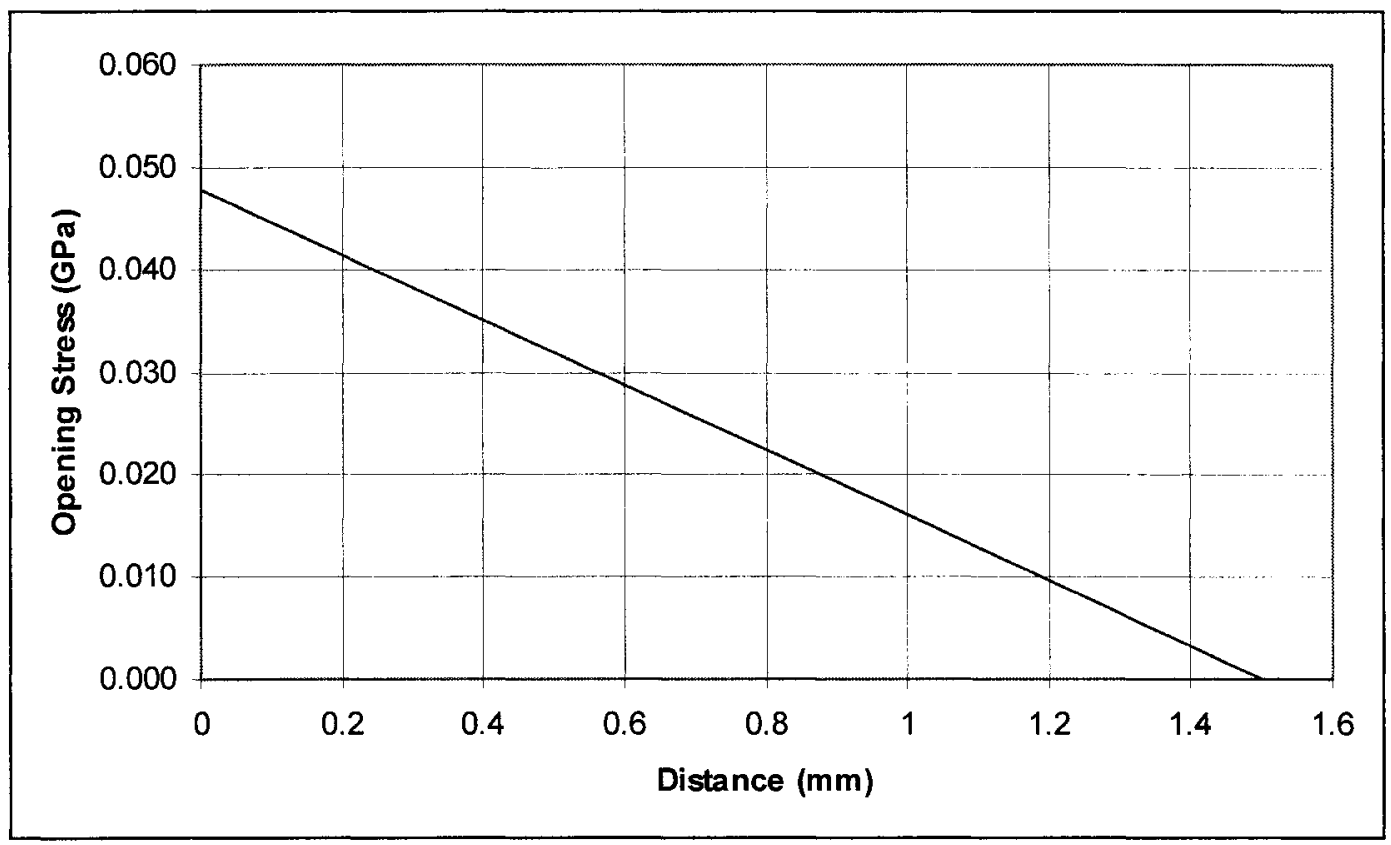

Figure 87: Stress-displacement correction curve.

A total of 31 simulations were carried out as shown in Table 27. This table summarizes the material models, interface types, element types, impact energy levels and simulation ID numbers. 
Table 27: Summary of GLARE low-velocity impact simulations.

\begin{tabular}{|c|c|c|c|c|c|}
\hline Simulation ID & Material & $\begin{array}{c}\text { Element } \\
\text { Type }\end{array}$ & $\begin{array}{c}\text { Energy } \\
\text { Level }\end{array}$ & Material Model & Interface Type \\
\hline 1 & GL-3-2/1 & Thick & 25 & Piecewise Linear/ Orthotropic & Tied \\
\hline 2 & GL-3-2/1 & Thick & 35 & Piecewise Linear/ Orthotropic & Tied \\
\hline 3 & GL-3-2/1 & Thick & 45 & Piecewise Linear/ Orthotropic & Tied \\
\hline 4 & GL-3-2/1 & Thick & 55 & Piecewise Linear/ Orthotropic & Tied \\
\hline 5 & GL-4-2/1 & Thick & 25 & Piecewise Linear/ Orthotropic & Tied \\
\hline 6 & GL-4-2/1 & Thick & 35 & Piecewise Linear/ Orthotropic & Tied \\
\hline 7 & GL-4-2/1 & Thick & 45 & Piecewise Linear/Orthotropic & Tied \\
\hline 8 & GL-4-2/1 & Thick & 55 & Piecewise Linear/ Orthotropic & Tied \\
\hline 9 & GL-5-2/1 & Thick & 25 & Piecewise Linear/ Orthotropic & Tied \\
\hline 10 & GL-5-2/1 & Thick & 35 & Piecewise Linear/ Orthotropic & Tied \\
\hline 11 & GL-5-2/1 & Thick & 45 & Piecewise Linear/ Orthotropic & Tied \\
\hline 12 & GL-5-2/1 & Thick & 55 & Piecewise Linear/ Orthotropic & Tied \\
\hline 13 & GL-3-2/1 & Thick & 25 & Piecewise Linear/ Orthotropic & Simple \\
\hline 14 & GL-3-2/1 & Thick & 35 & Piecewise Linear/ Orthotropic & Simple \\
\hline 15 & GL-3-2/1 & Thick & 55 & Piecewise Linear/ Orthotropic & Simple \\
\hline 16 & $G L-4-2 / 1$ & Thick & 35 & Piecewise Linear/ Orthotropic & Simple \\
\hline 17 & $\mathrm{GL}-4-2 / 1$ & Thick & 45 & Piecewise Linear/ Orthotropic & Simple \\
\hline 18 & $G L-4-2 / 1$ & Thick & 55 & Piecewise Linear/ Orthotropic & Simple \\
\hline 19 & $G L-3-2 / 1$ & Thick & 25 & Piecewise Linear/ Orthotropic & Corrected \\
\hline 20 & GL-3-2/1 & Thick & 35 & Piecewise Linear/ Orthotropic & Corrected \\
\hline 21 & GL-3-2/1 & Thick & 45 & Piecewise Linear/ Orthotropic & Corrected \\
\hline 22 & GL-3-2/1 & Thick & 25 & Piecewise Linear /UMAT & Simple \\
\hline 23 & GL-3-2/1 & Thick & 35 & Piecewise Linear /UMAT & Simple \\
\hline 24 & GL-3-2/1 & Thick & 45 & Piecewise Linear/UMAT & Simple \\
\hline 25 & $G L-4-2 / 1$ & Thick & 35 & Piecewise Linear/UMAT & Simple \\
\hline 26 & $G L-4-2 / 1$ & Thick & 45 & Piecewise Linear/UMAT & Simple \\
\hline 27 & $G L-4-2 / 1$ & Thick & 55 & Piecewise Linear/UMAT & Simple \\
\hline 28 & GL-5-2/1 & Thick & 35 & Piecewise Linear/UMAT & Simple \\
\hline 29 & GL-5-2/1 & Thick & 45 & Piecewise Linear/UMAT & Simple \\
\hline 30 & GL-5-2/1 & Thick & 55 & Piecewise Linear/UMAT & Simple \\
\hline 31 & GL-5-2/1 & Thick & 65 & Piecewise Linear/UMAT & Simple \\
\hline
\end{tabular}




\subsection{Mesh Sensitivity}

A mesh density study was conducted using GLARE-3-2/1 simulations with three mesh density levels. These simulations used the UMAT material model for the prepreg with the simple-tiebreak interface model for initiation of delamination. The basic simulation parameters are presented in Table 28. The remaining parameters were presented in the previous section in Figures 85 to 87 and Tables 25 and 26.

Table 28: Summary of GLARE mesh density simulation parameters.

\begin{tabular}{|c|c|c|c|c|}
\hline \multirow{2}{*}{ Run ID } & \multirow{2}{*}{ \# of Elements } & \multirow{2}{*}{ \# of Nodes } & \multirow{2}{*}{ \# of Cycles } & CPU Time \\
\cline { 4 - 5 } & & 3437 & 270979 & $(\mathbf{s})$ \\
\hline 1-low & 1636 & 5229 & 270609 & 17644 \\
\hline 2-medium & 2500 & 11389 & 273158 & 27644 \\
\hline 3-high & 5500 & & 68716 \\
\hline
\end{tabular}

As can be seen in Figure 88, there was little effect of mesh density on the predicted levels of absorbed energy. The agreement between the three mesh densities was also apparent from the force versus time curves plotted in Figure 89. It is only for the deflection curves in Figure 90 that some difference in the response of the three panels is observed. The low-density mesh shows less permanent deflection than the mediumand high-density meshes. 


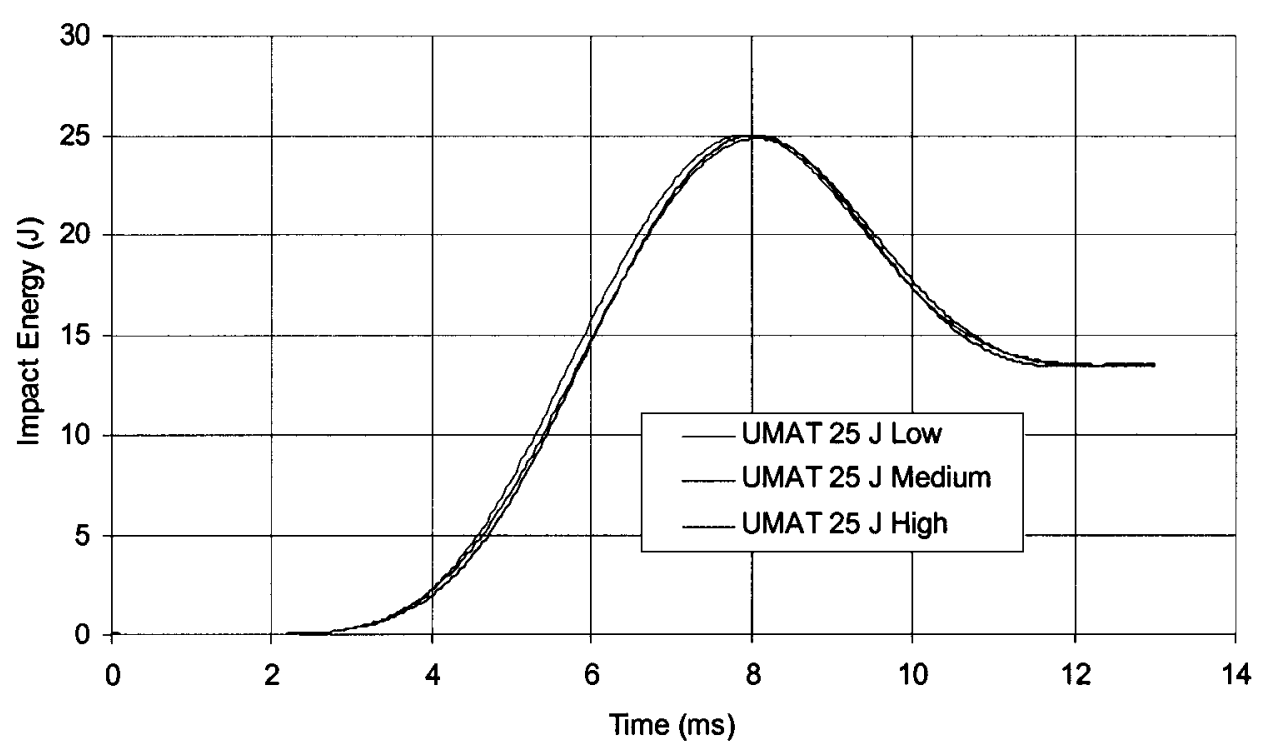

Figure 88: Impact energy versus time for three different mesh densities of a GLARE-3 impact simulation.

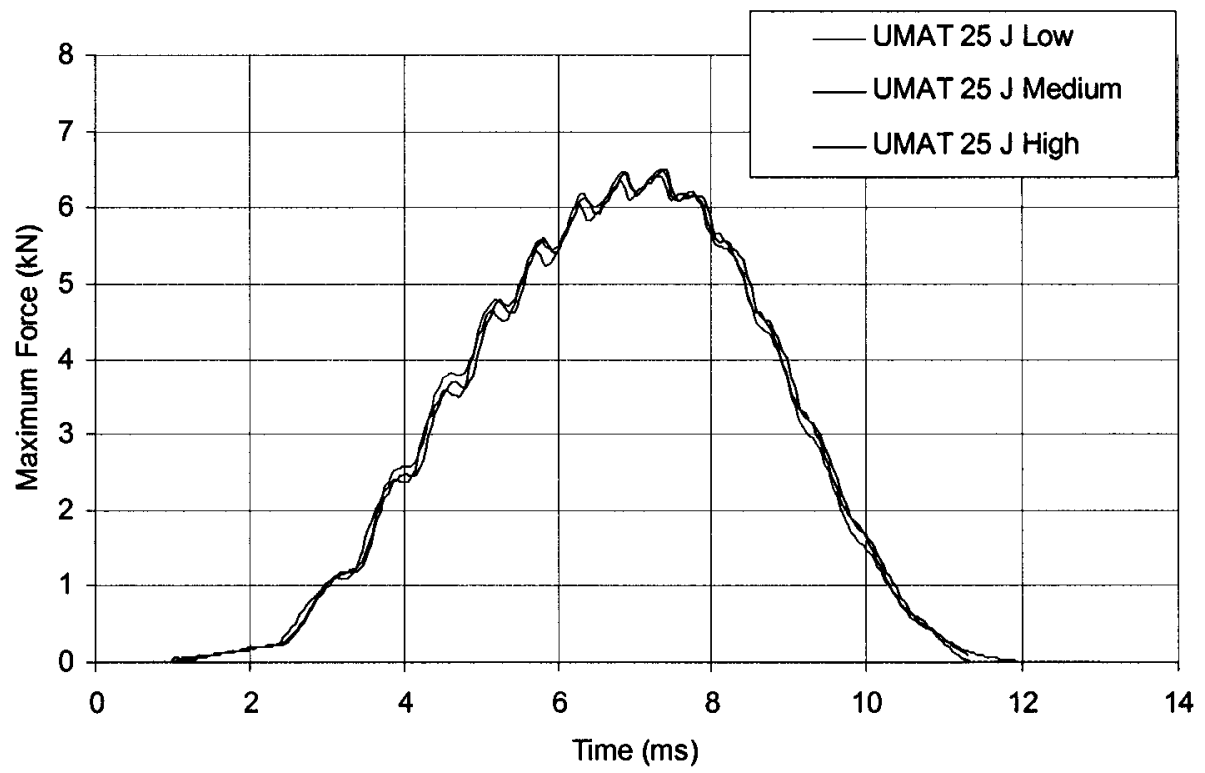

Figure 89: Impact force versus time for three different mesh densities of a GLARE-3 impact simulation. 


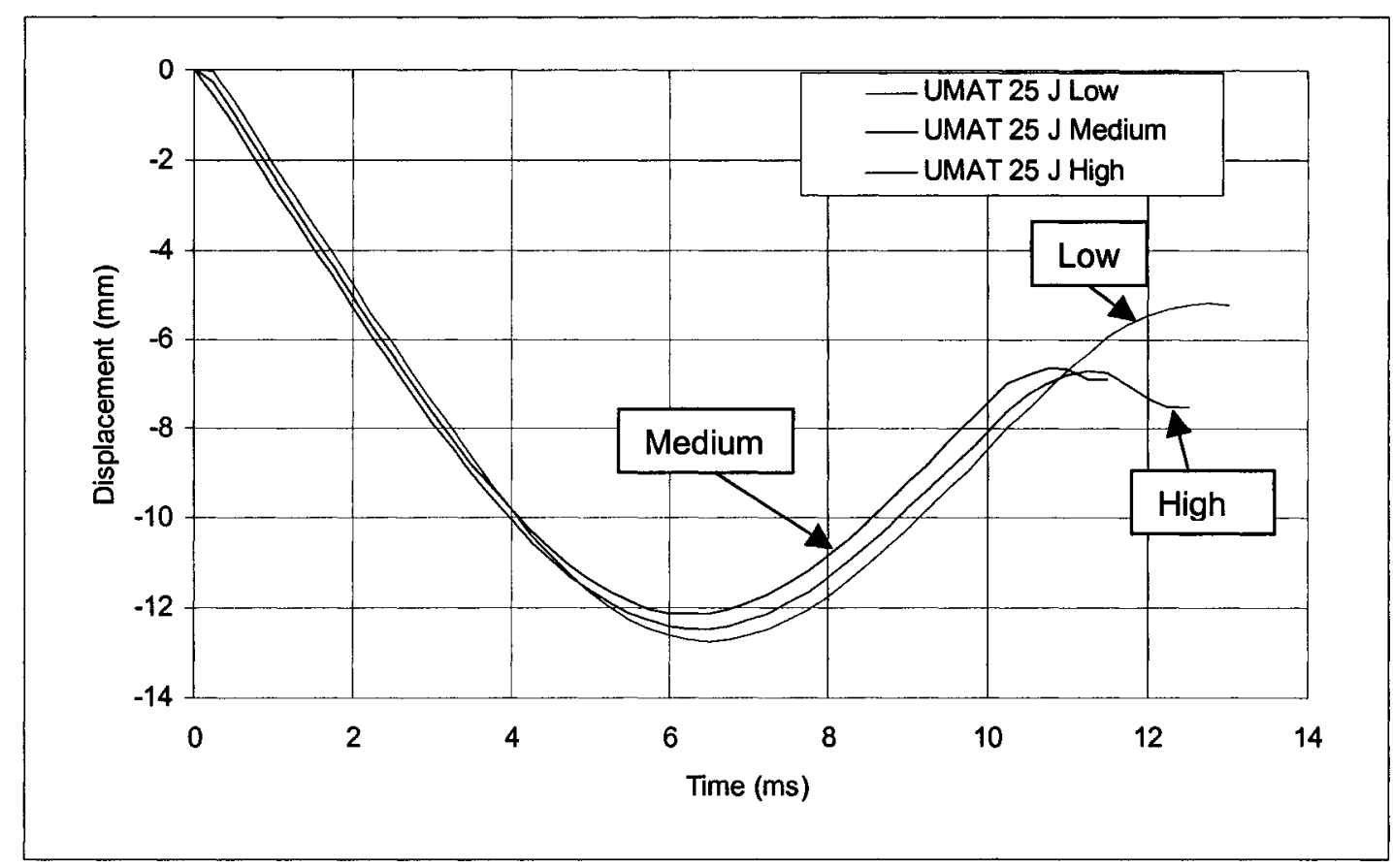

Figure 90: Dent depth versus time for three different mesh densities with GLARE-3.

The predicted damaged panel geometries are shown in Figure 91. The mediumand high-density meshes give similar predicted damage geometries. However, the lowdensity mesh showed larger amounts of delamination damage. The smaller number of nodes in the low-density simulation is likely the cause of the increased delamination. There are fewer nodes to be connected by the simple-tiebreak interface delamination criterion. A large area of delamination is created when one node is released after the failure criterion is reached. As the number of nodes decreases, the size of the delaminated region increases when a single node is released. Another effect of larger elements is that the damage is distributed through fewer elements. Therefore, a single element would have to accumulate a greater amount of damage before failing. Based on this study, the medium density mesh was selected for the remainder of the GLARE impact simulations. 

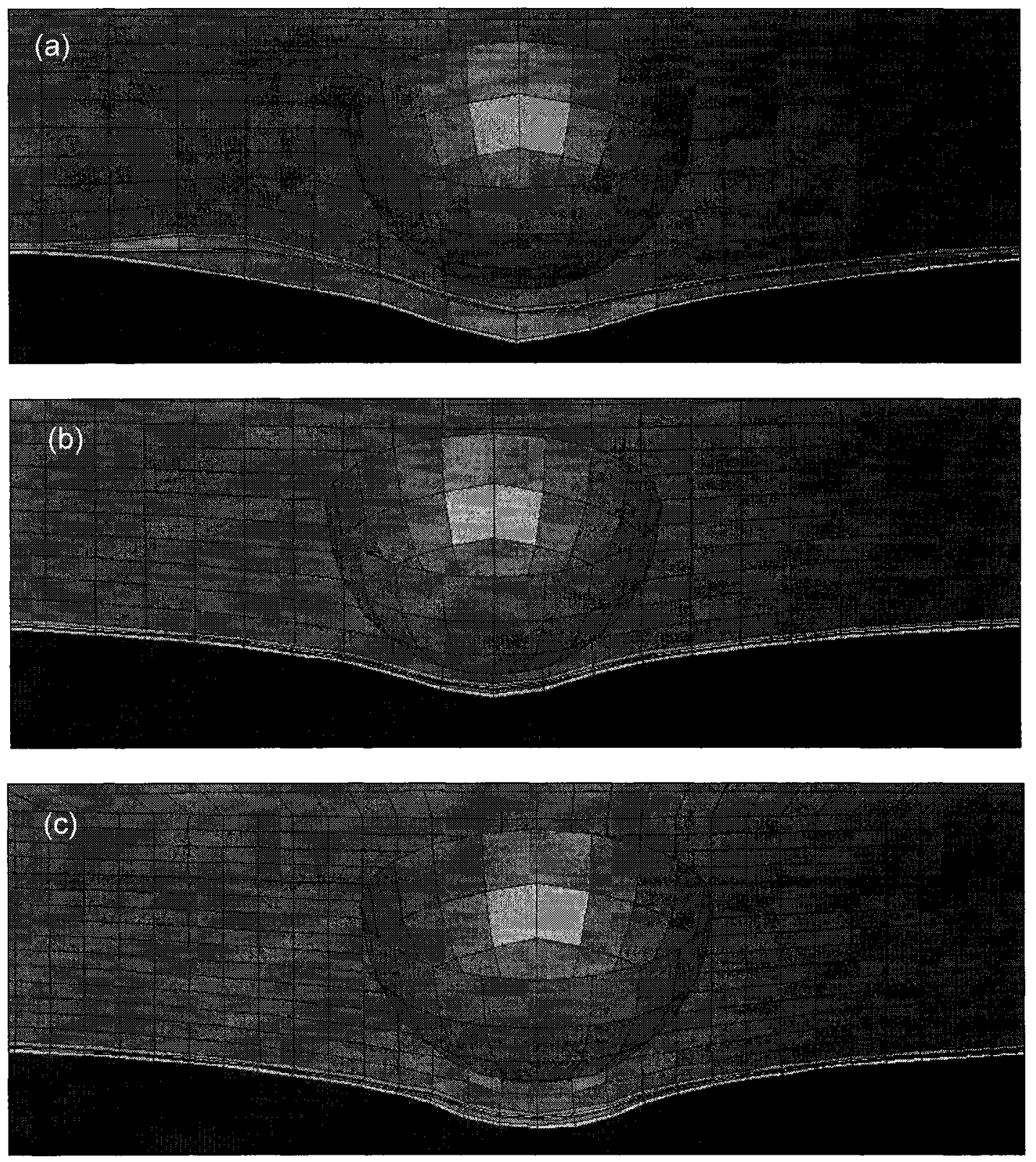

Figure 91: Predicted impacted panel geometries for (a) low-, (b) medium- and (c) high-density meshes. 


\subsection{Simulation Results}

The measured and predicted absorbed energy, dent depth and peak force results are presented in the following sections. These damage comparisons include maps of the delamination damage in the simulations and experiments. A complete tabulation of the simulation results is provided in Appendix G. Note that the steps near the end of each set of experimental data are due to puncture damage developing in some of the impacted panels.

\subsubsection{Absorbed Energy}

Typical energy versus time plots from the simulations and experiments conducted using GLARE-3 impacted at $25 \mathrm{~J}$ are shown in Figure 92. The simulations performed using the orthotropic-elastic prepreg (type 2) predicted lower absorbed energies than was measured in the experiments. However, the simulation that used the UMAT showed a definite improvement in the predicted amount of absorbed energy. One possible source of the remaining difference between the predictions and the experiments is the method by which the energy is calculated by the drop tower software. The experimental impact energy is calculated directly from the impact force (Equations 1-4). Thus, the experimental energy would include any system losses. Further differences could also result from the experimental boundary condition that is between simply-supported and clamped. 


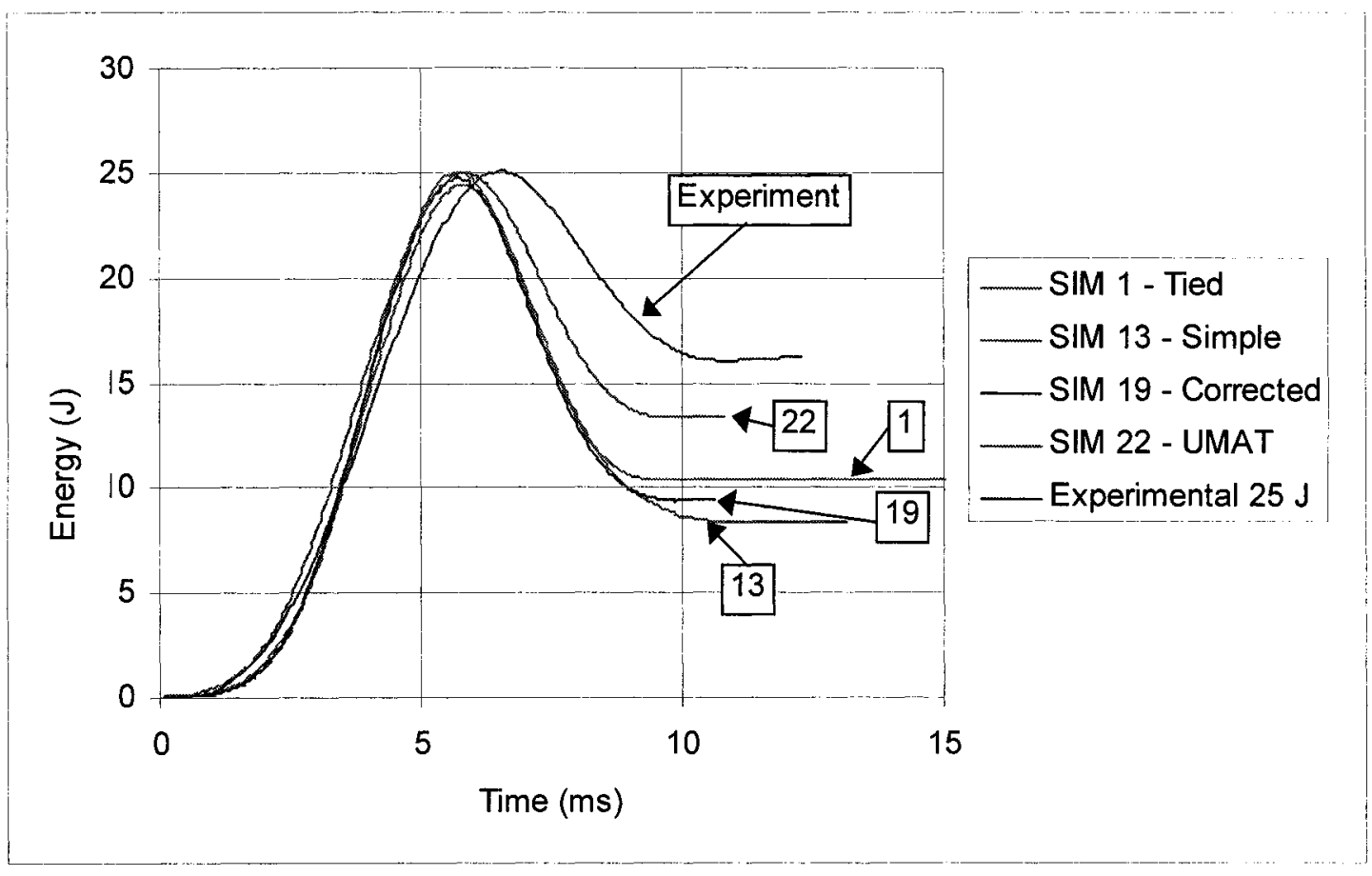

Figure 92: Typical absorbed energy versus time curves for simulations and experiments with GLARE-3 impacted at $25 \mathrm{~J}$.

Figures 93 to 95 summarize the results of all of the simulations for GLARE-3, GLARE-4 and GLARE-5 and compare the values with the experiments. However, the predicted values were still lower than the experimental values. A similar discrepancy was observed for the aluminum simulations (Chapter 4). The lack of fixture losses could also be affecting the GLARE simulations. The simple-tiebreak interface simulations showed the lowest absorbed energy of all types. This is due to the rebound of the prepreg layers, an action that releases stored elastic energy from these layers. 


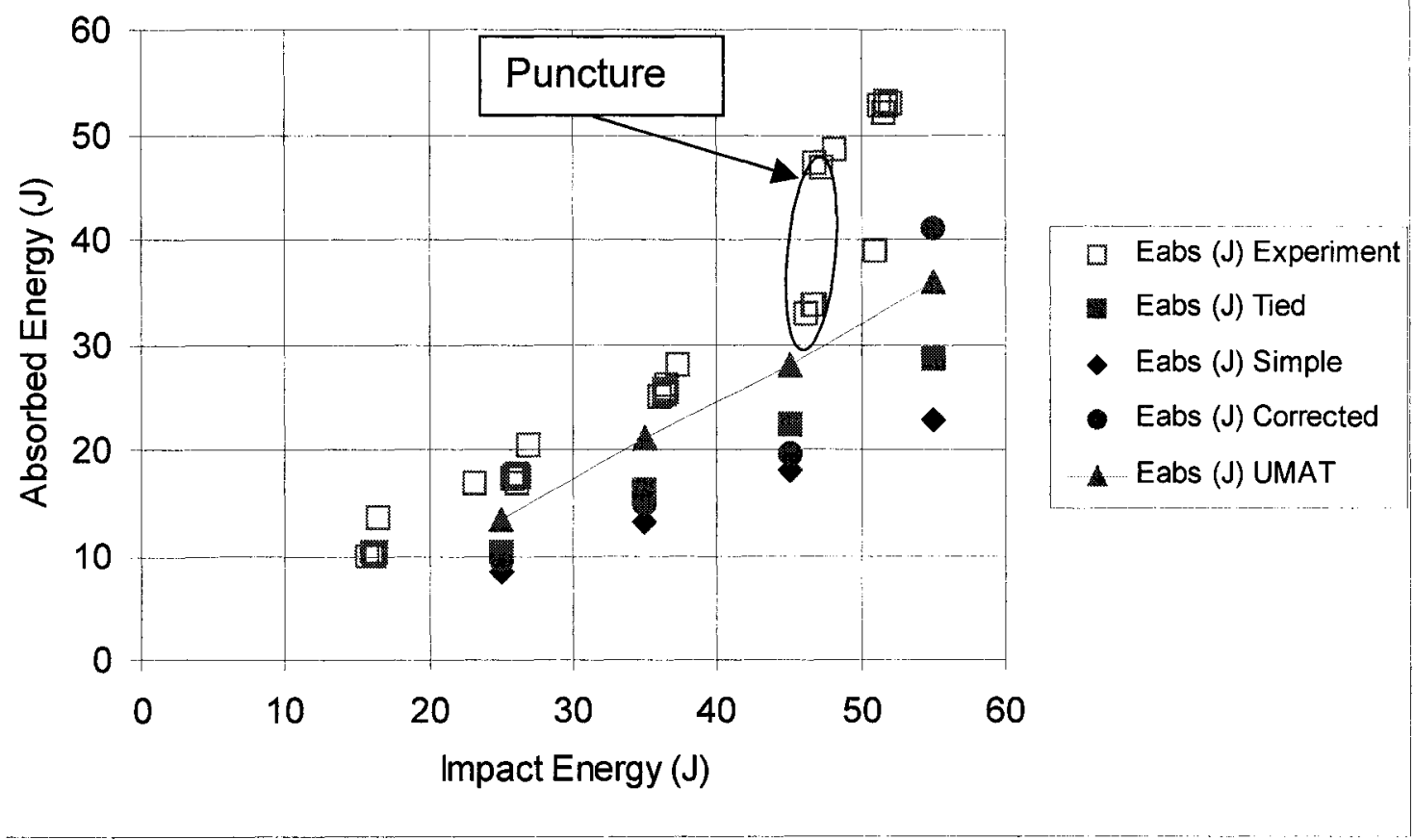

Figure 93: Absorbed Energy versus Impact Energy for GLARE-3.

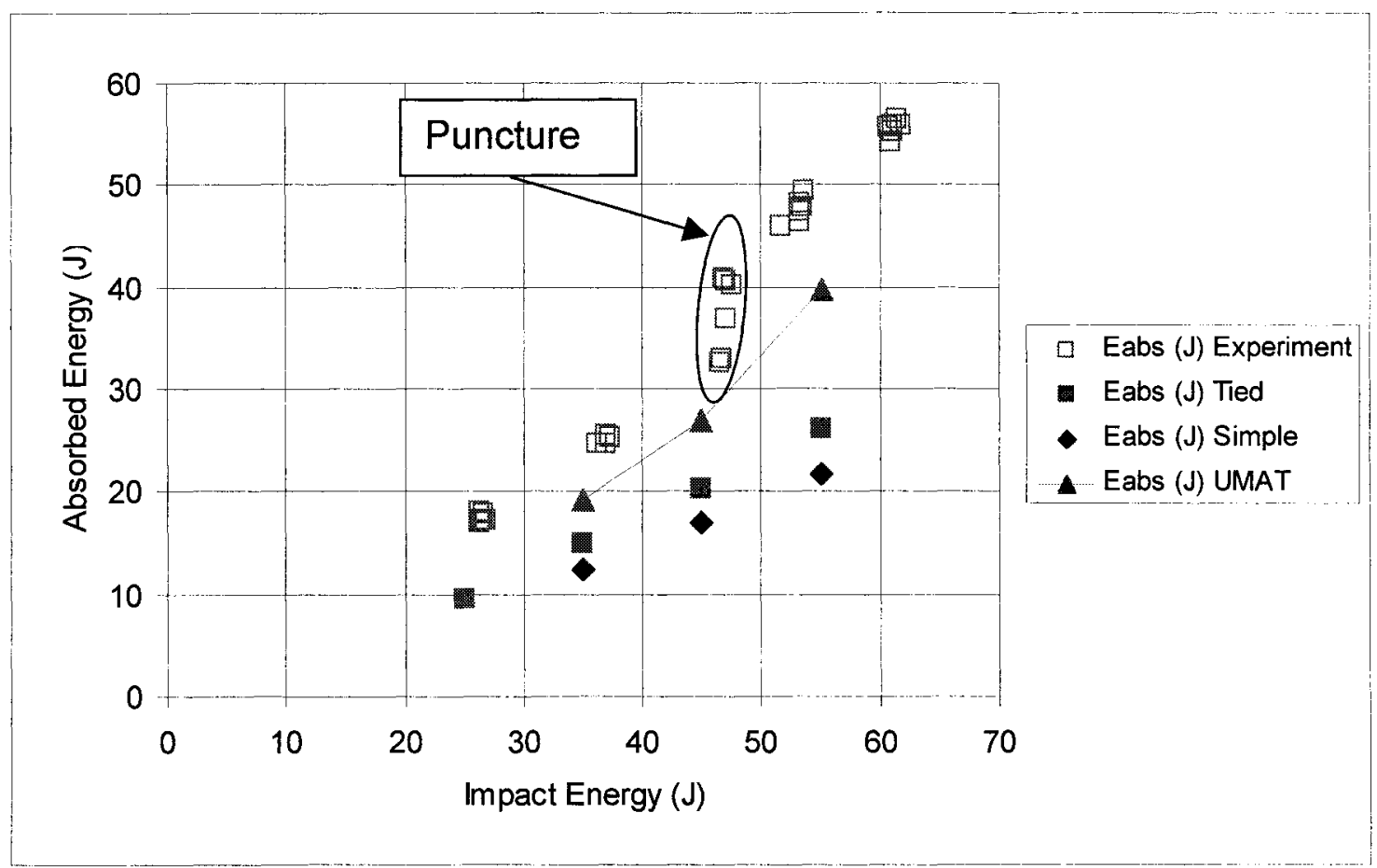

Figure 94: Absorbed Energy versus Impact Energy for GLARE-4. 


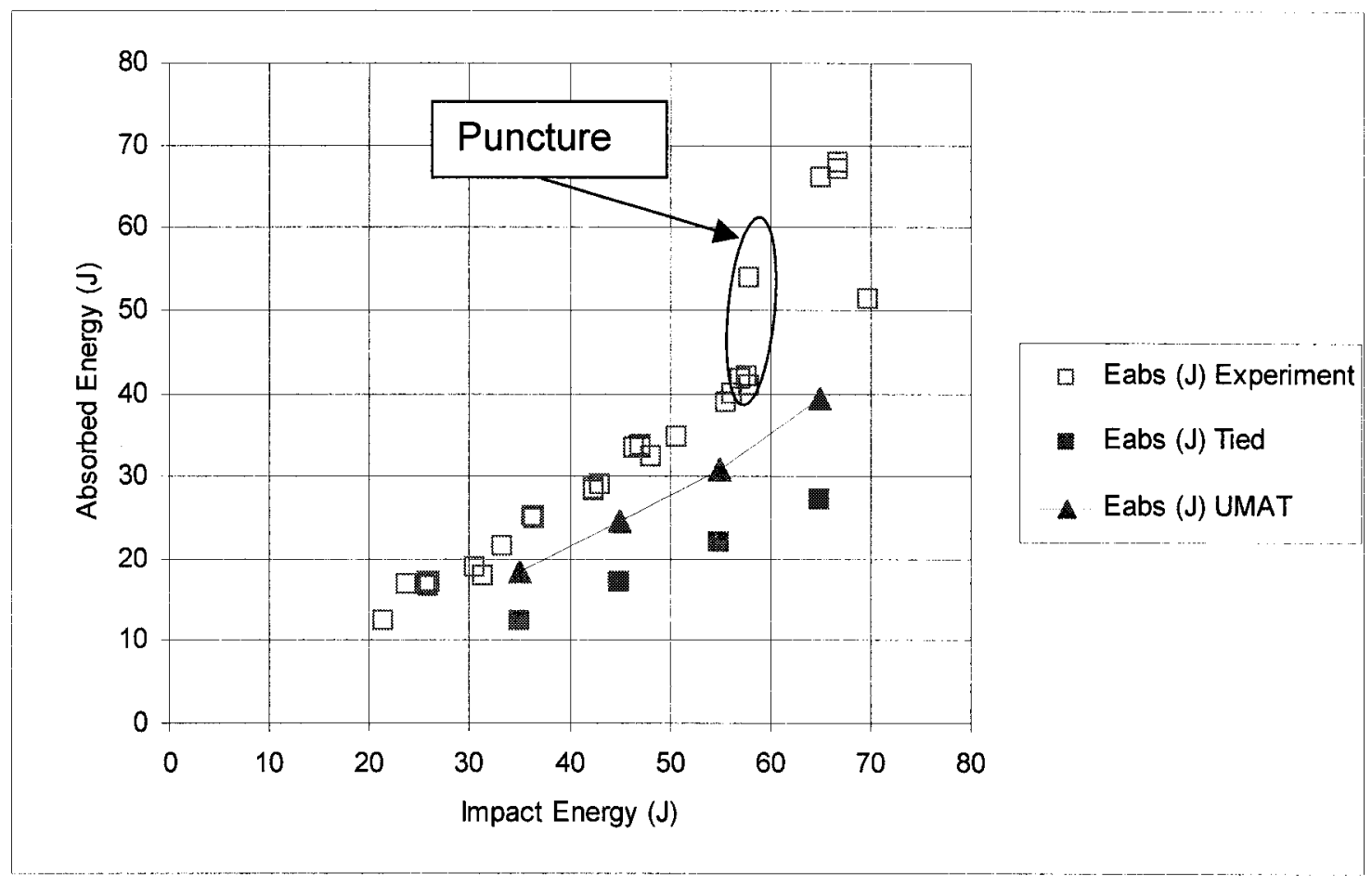

Figure 95: Absorbed Energy versus Impact Energy for GLARE-5.

\subsubsection{Permanent Dent Depth}

A plot of the typical displacements measured for GLARE-3-2/1 impacted at $25 \mathrm{~J}$ is shown in Figure 96. Note that the experimental displacement curve is calculated as per Equations 1-4 from the impact force data by the drop tower software. The experimental set-up did not include equipment to measure the displacement of the panel during impact. However, the dent depths from the simulations are direct measurements of nodal displacements at the point of impact. The UMAT simulations predict higher dent depths than the other simulations. As shown below, the permanent dent depth measurements from the experiments agree favourably with the UMAT simulations. 


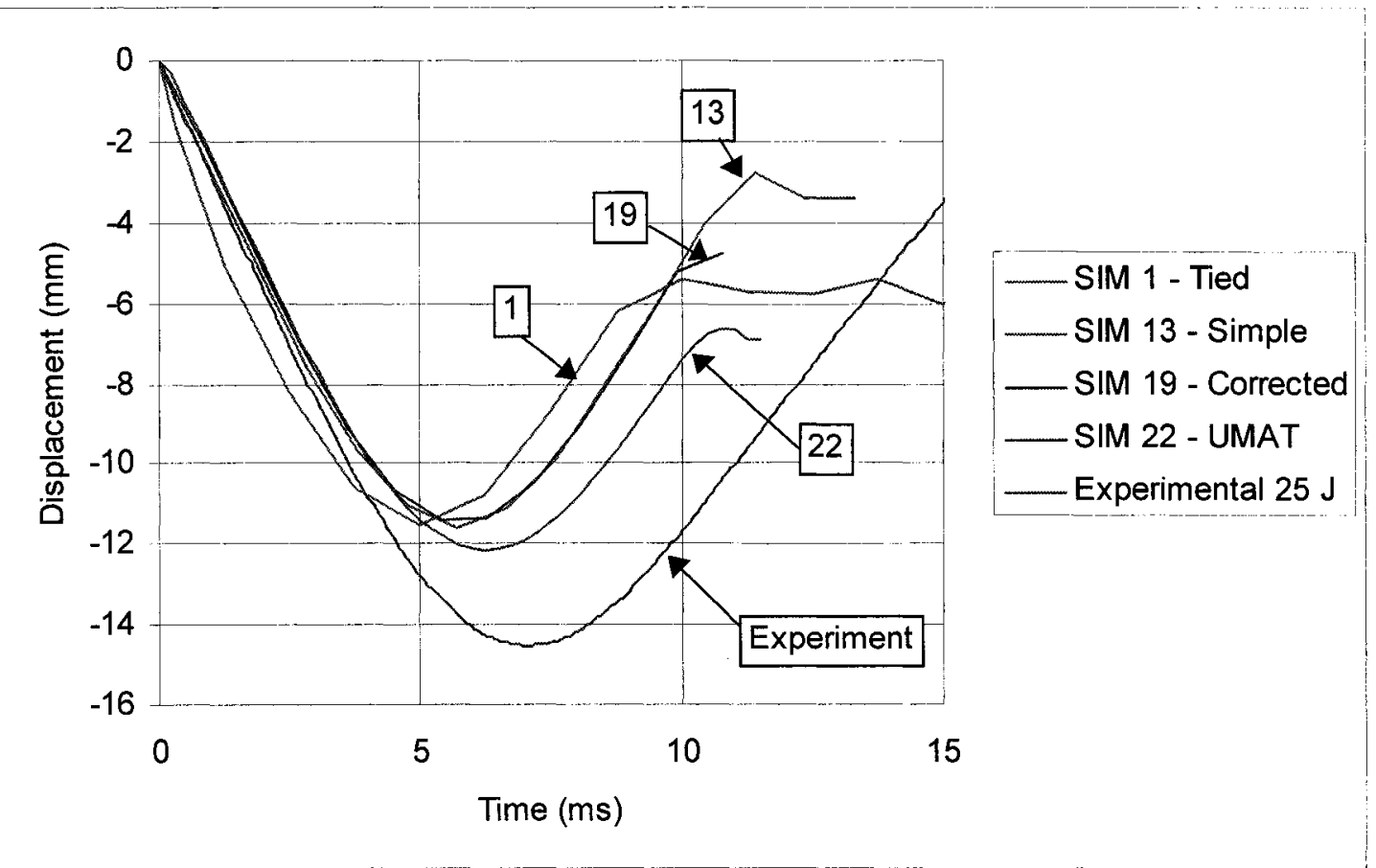

Figure 96: Typical dent depth versus time plots for GLARE-3-2/1 impacted with $25 \mathrm{~J}$.

The effects of including the UMAT subroutine on the predictions of permanent dent depth are shown in Figure 97 to 99 . The simple-tiebreak interface dent depth results were significantly lower than the other simulation results and the experimental values. The permanent dent depth from the experiments was measured directly from the panels using a vernier depth gauge. 


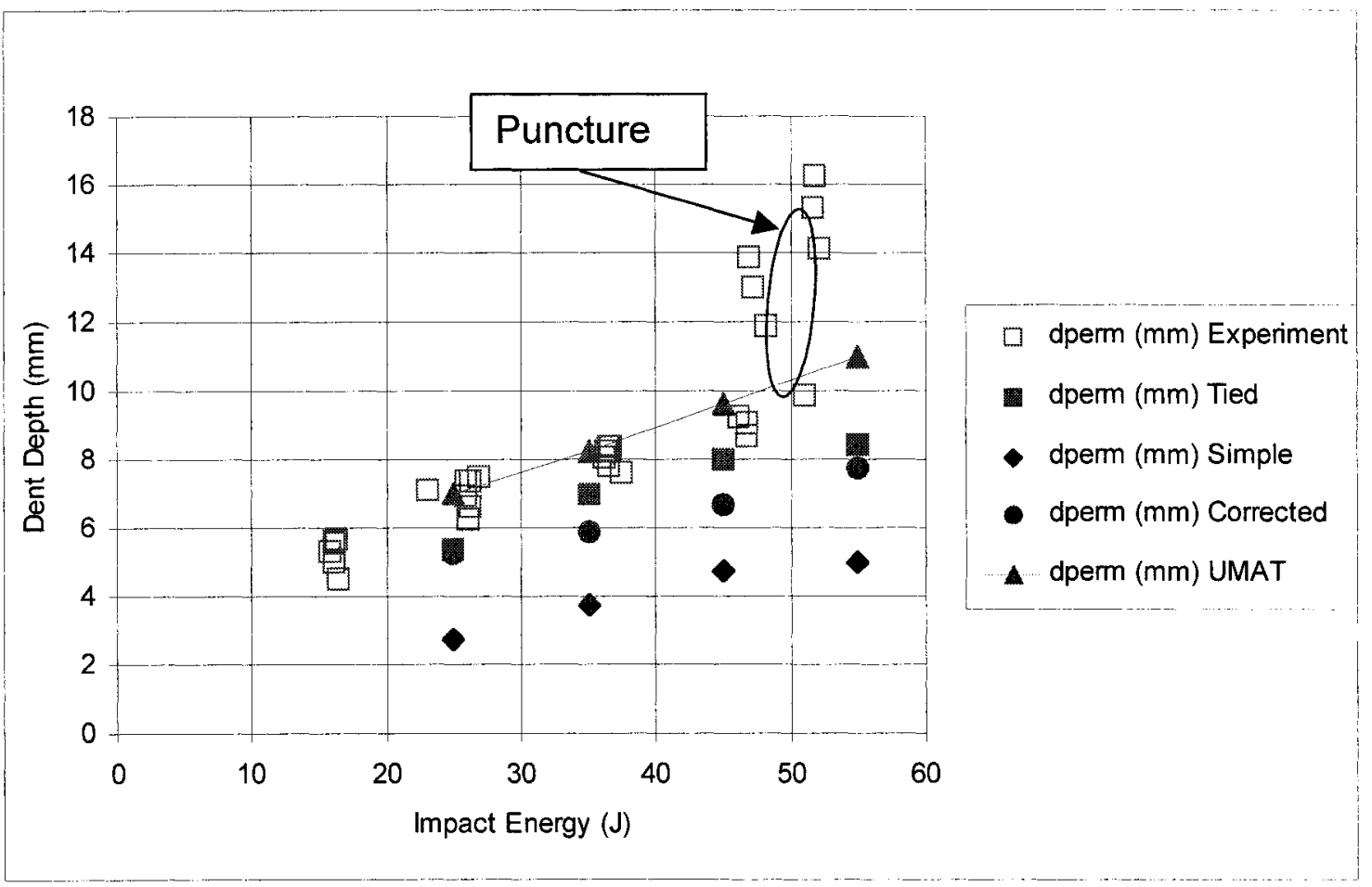

Figure 97: Dent Depth versus Impact Energy for GLARE-3.

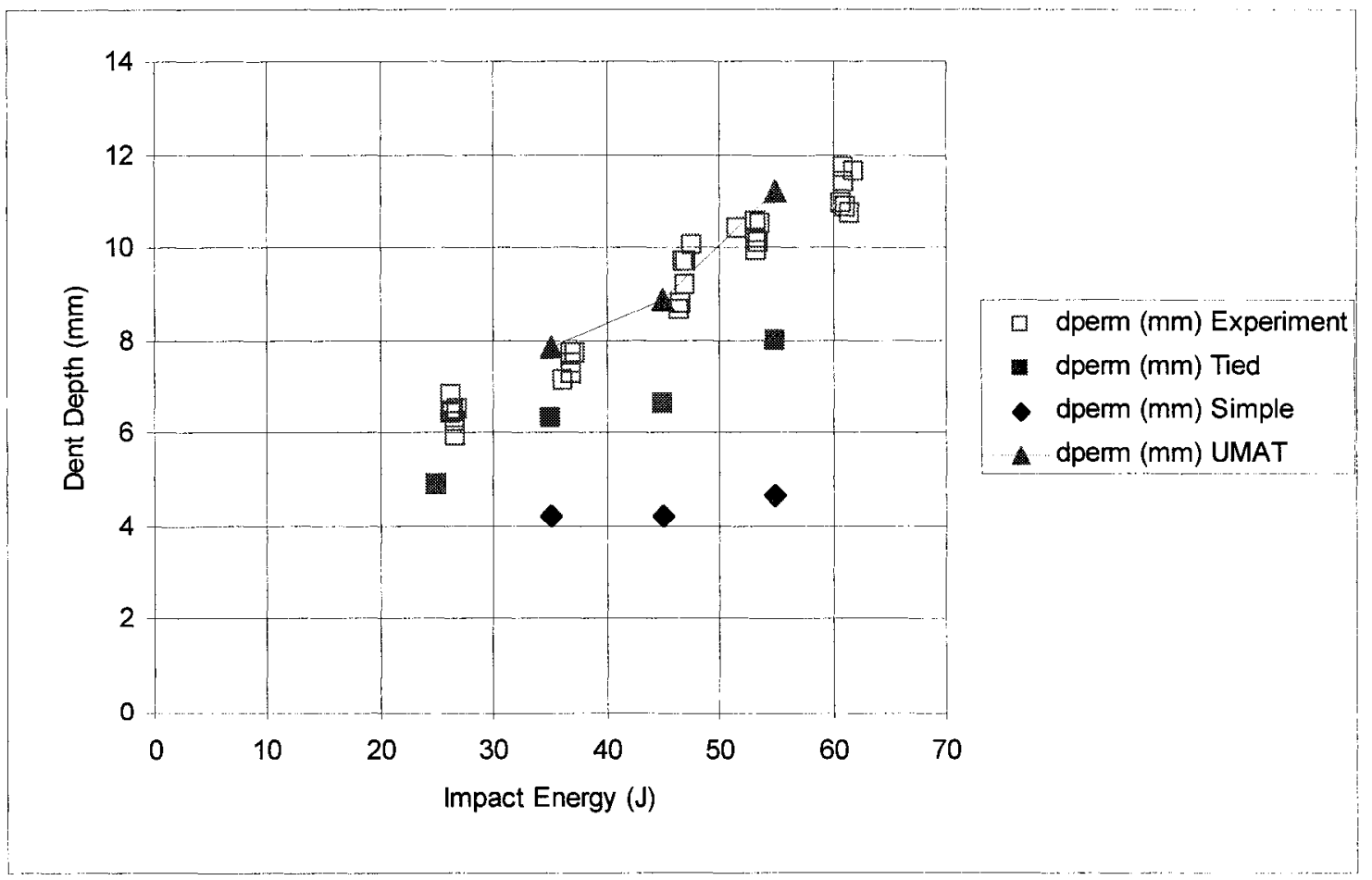

Figure 98: Dent Depth versus Impact Energy for GLARE-4. 


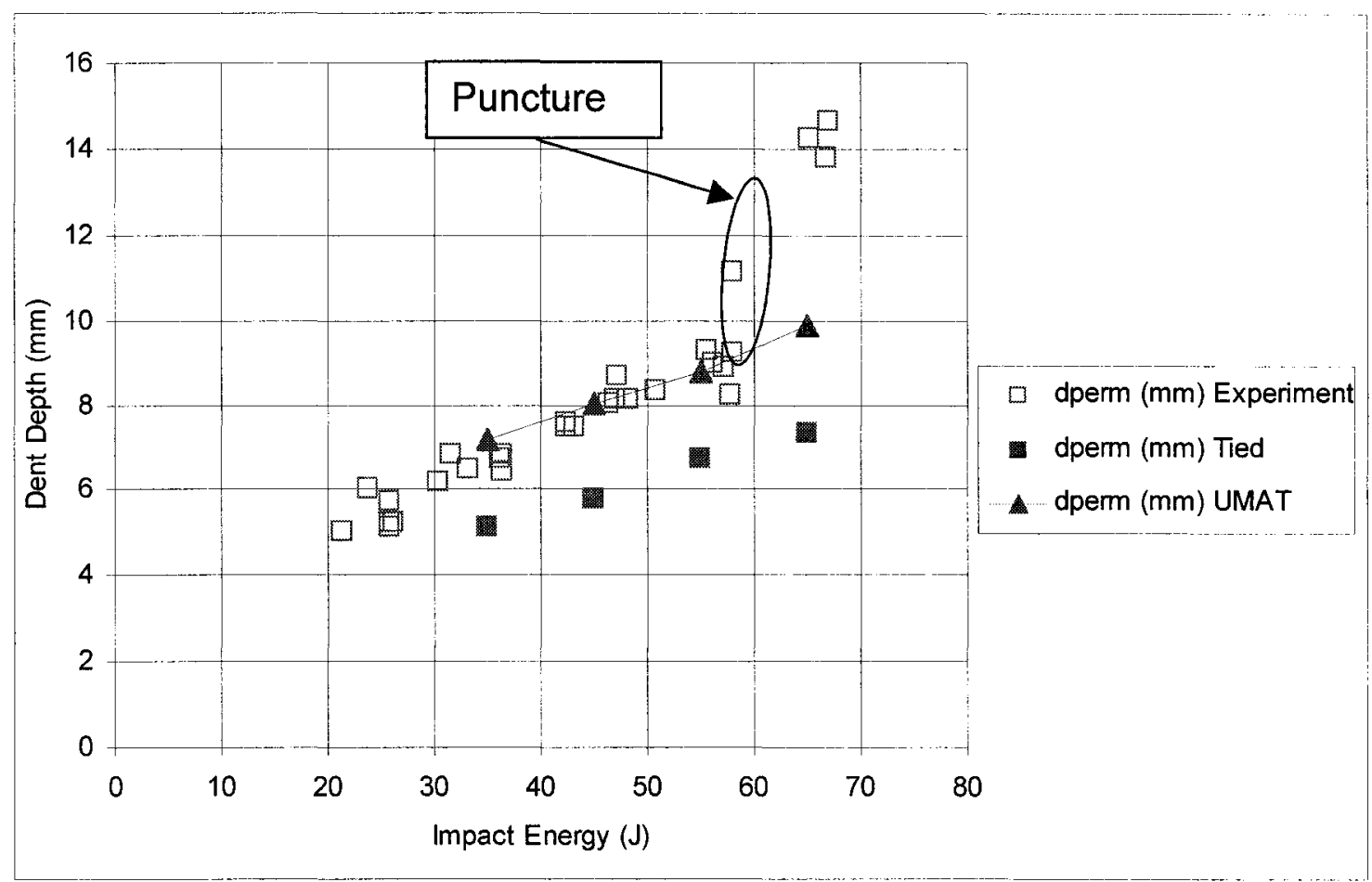

Figure 99: Dent Depth versus Impact Energy for GLARE-5.

\subsubsection{Peak Impact Force}

The last comparison between the GLARE simulation results and the experiments is the peak impact force. This is the peak force measured between the specimen and the impactor during impact. Plots of the typical impact force versus time measurements for the simulations and experiments with GLARE-3-2/1 impacted at $25 \mathrm{~J}$ are shown in Figure 100. The simulations conducted using the UMAT model gave a lower peak impact force than the other simulations. However, the forces were still higher than those measured during the experiments. The experiments showed less vibrations than the simulations. Damping in the structure of the crosshead, where the load is measured, could be the cause of this effect. 


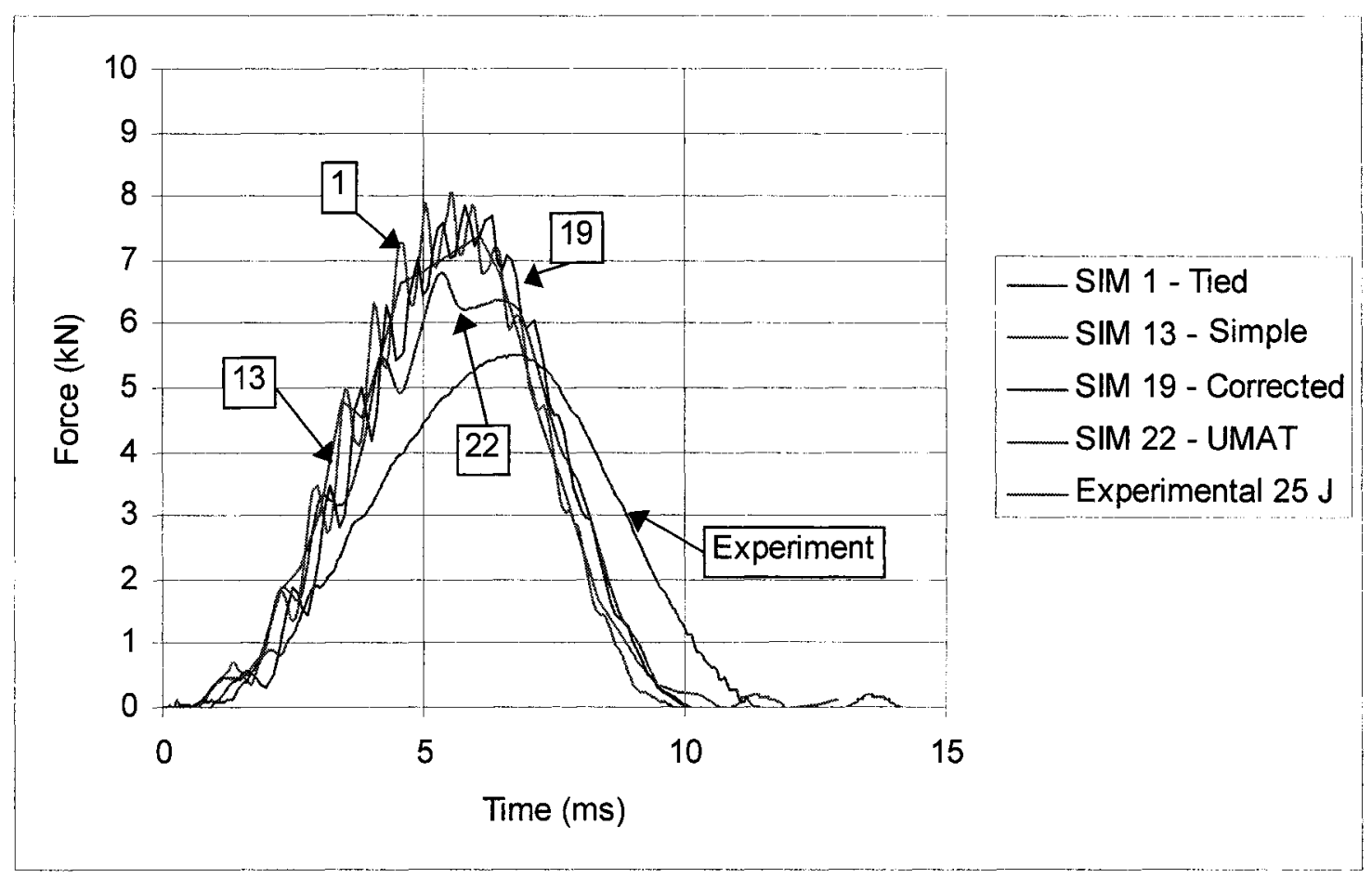

Figure 100: Typical impact force versus time plots for GLARE-3-2/1 impacted at $25 \mathrm{~J}$.

The tied-, simple-tiebreak, and corrected-tiebreak interface models, shown in Figures 101 to 103 , all over-predict the peak impact force to approximately the same degree. These models use an elastic model for the prepreg layers. In the UMAT model, the prepreg layer loses stiffness as it accumulates damage and the panel is not able to resist the penetration of the impactor to the same degree as it does with the elastic material model. While the UMAT-based simulations still over-predict the impact force, they are an improvement over the other three techniques. 


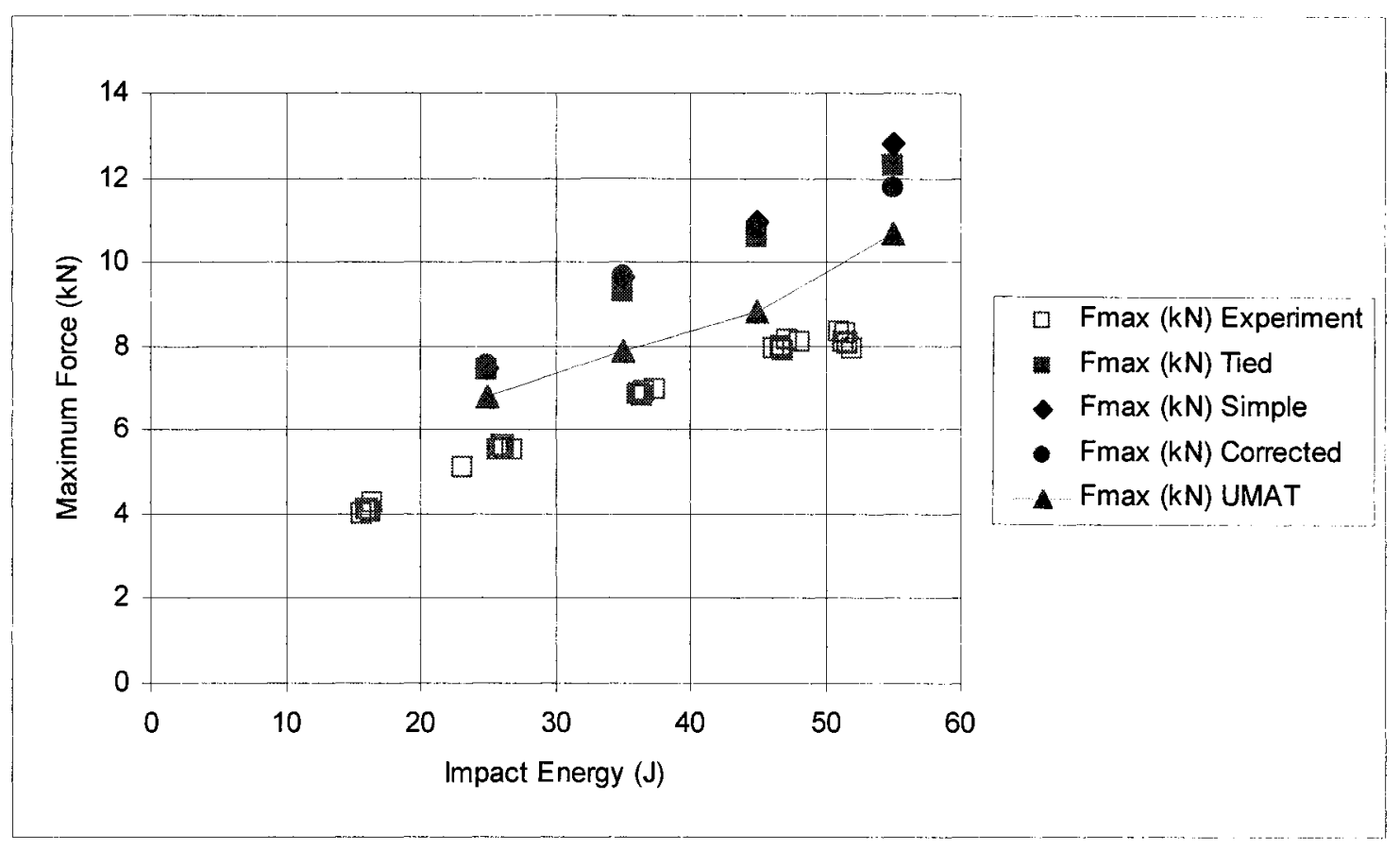

Figure 101: Maximum Force versus Impact Energy for GLARE-3.

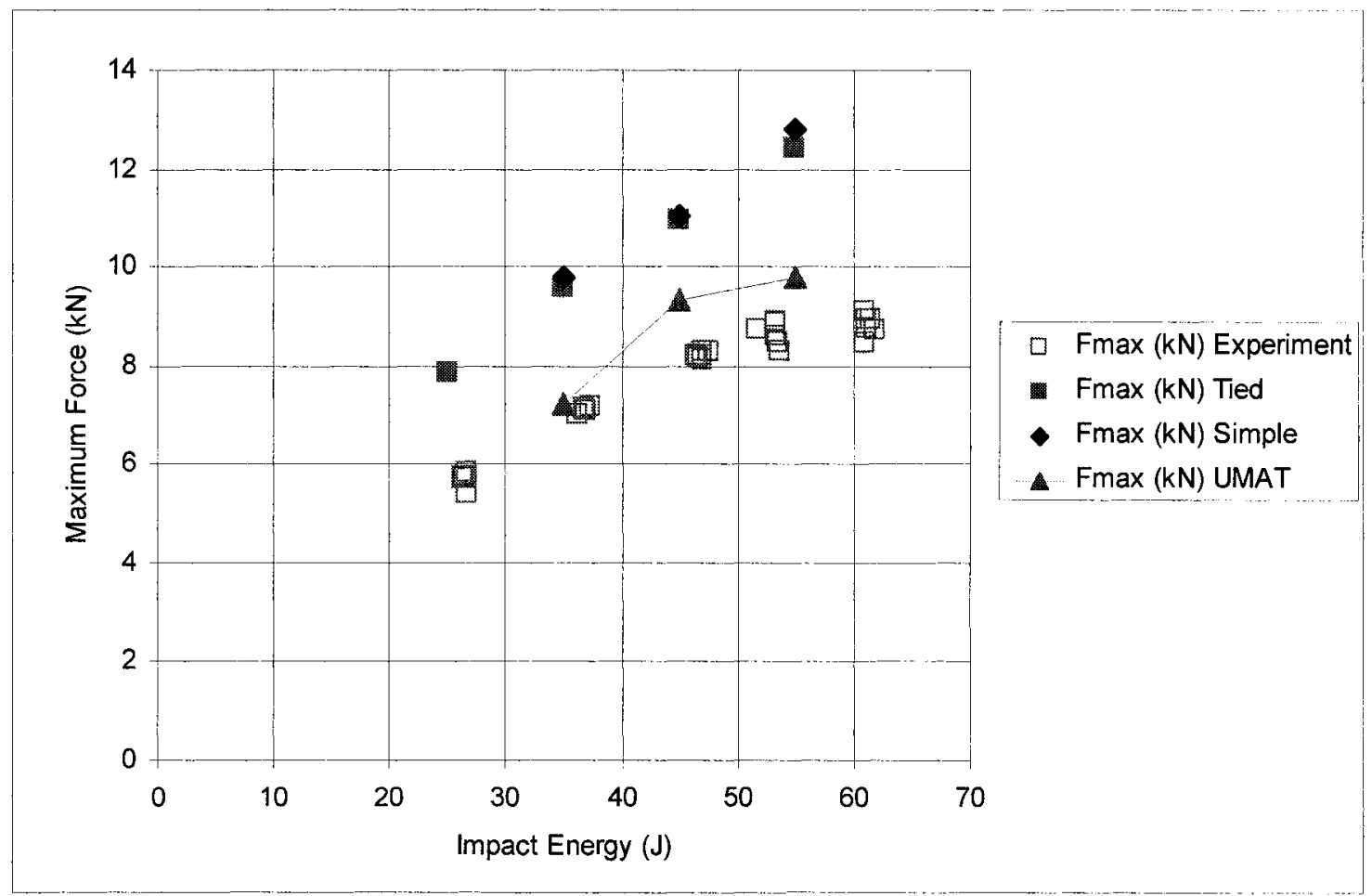

Figure 102: Maximum Force versus Impact Energy for GLARE-4. 


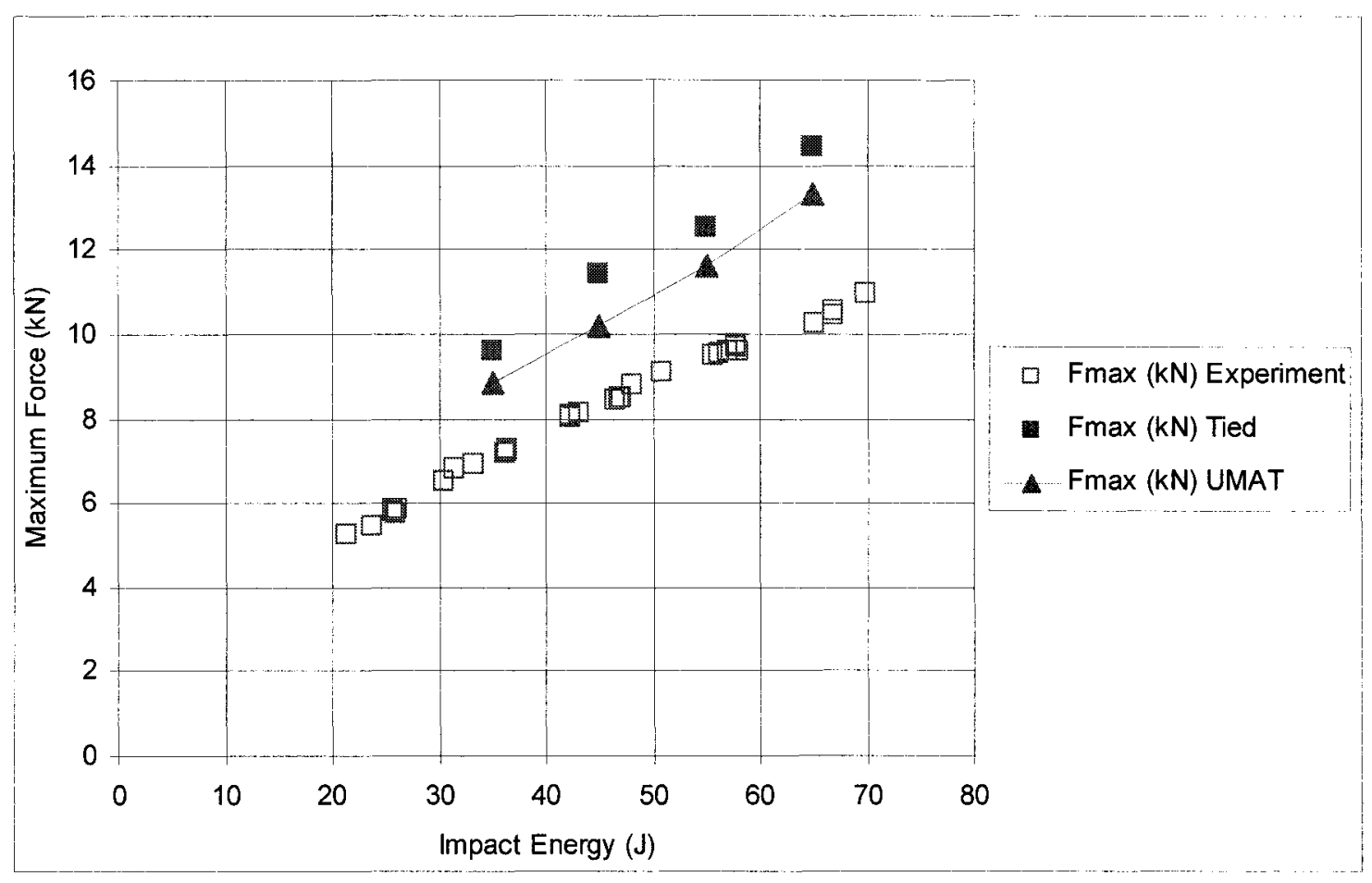

Figure 103: Maximum Force versus Impact Energy for GLARE-5.

\subsubsection{Predicted Damage Geometry}

Figure 104 shows delamination damage recorded during the experimental impact testing of GLARE-3. Images of the typical predicted delamination damage for each type of simulation are shown in Figure 105a-d. Figure 105a shows the typical damage geometry predicted using the tied-interface. There is no delamination predicted using this interface model.

As shown in Figure 105b, using the simple-tiebreak interface with an elastic prepreg resulted in the largest difference in predicted geometry. These simulations were first attempted using the manufacturer's adhesive property data $\left(s_{n}=48 \mathrm{MPa}\right.$ and $s_{s}=30$ $\mathrm{MPa}$ ). However, it was found that no delamination damage was predicted in the panels using these values. Reduced strengths were simulated until it was found that a value of 
approximately 0.025 of that suggested by the manufacturer resulted in the formation of delamination damage.

As described in Section 3.3.5, a correction curve can be used to modify the separation behaviour of the layers in LS-DYNA. An image of the typical correctedtiebreak interface delamination damage is shown in Figure 105c. This correction did improve the predictions of the damage geometry but did not improve the predicted response of the panels. Figure $105 \mathrm{~d}$ shows the typical geometry predicted using the simple-tiebreak interface model with the UMAT material model. The damage geometry is closer to the experiments compared to the simple-tiebreak interface model and the predicted response of the panels is improved as well.

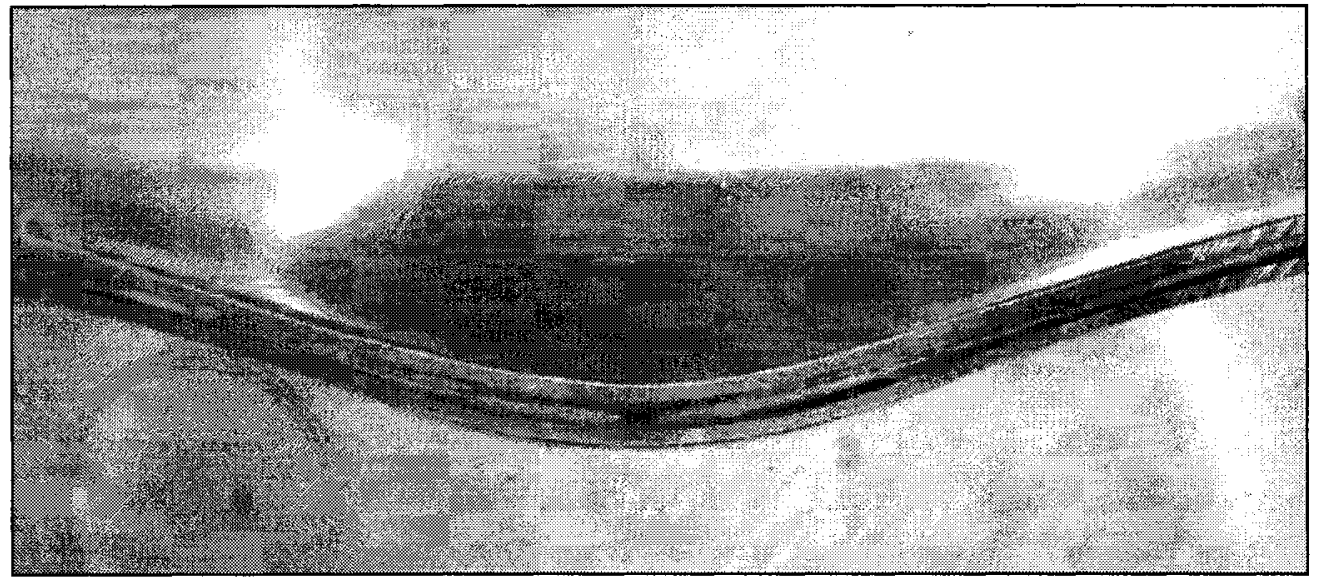

Figure 104: Delamination damage in GLARE-3 impacted at $\sim 45 \mathrm{~J}$. 


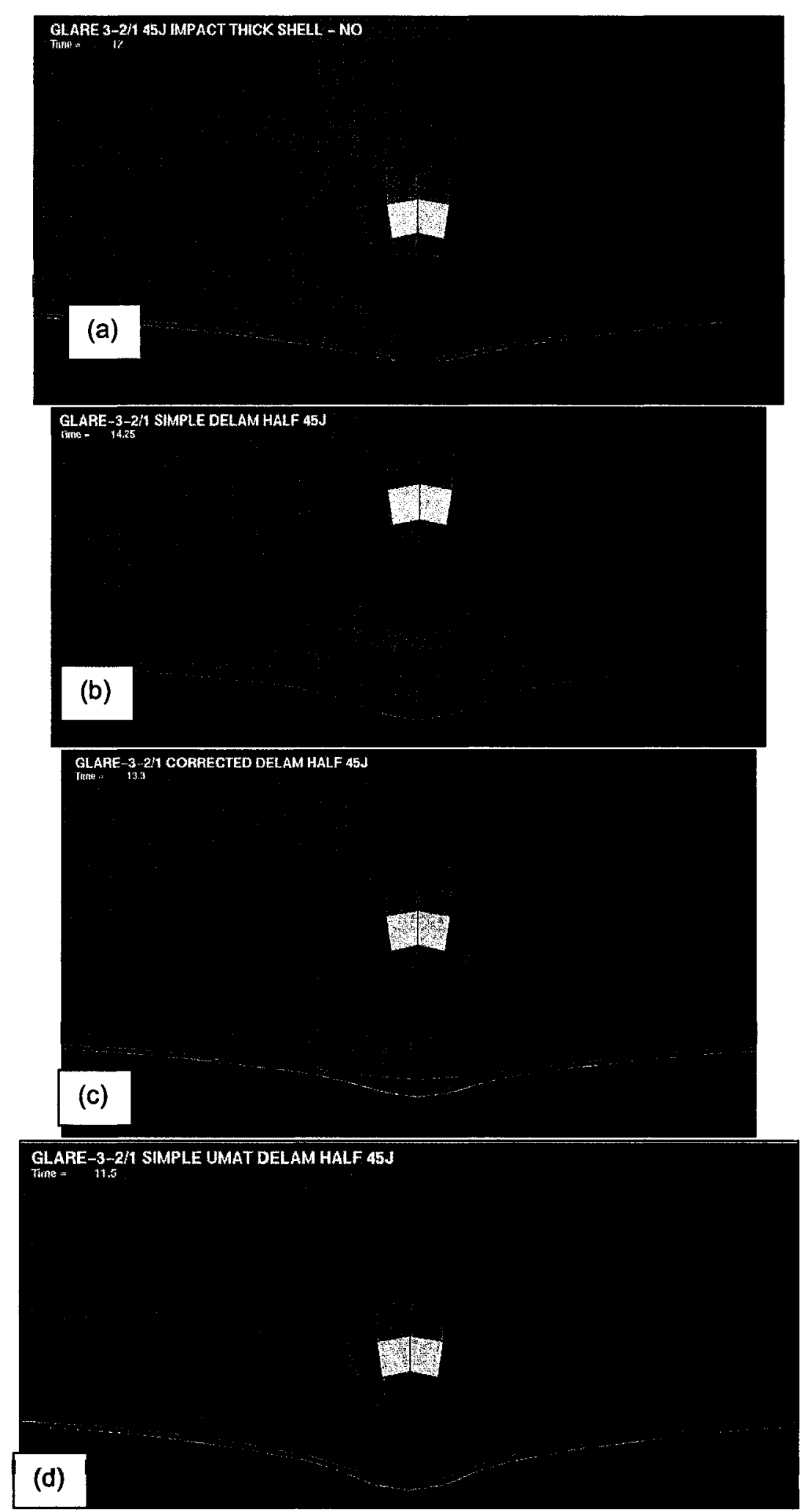

Figure 105: Typical delamination damage predicted using the (a) tiedinterface, (b) simple-tiebreak interface, (c) corrected-tiebreak interface and (d) UMAT/simple-tiebreak interface models. 
Figures 106 to 113 contain maps of the delamination damage predicted in the simulations with comparisons to the damage observed in the $\mathrm{x}$-rays and the destructive inspections. The damage maps were created by examining the geometry of the final simulations and locating each delaminated element. Note that each small square in the damage maps is $3.75 \mathrm{~mm} \times 3.75 \mathrm{~mm}$. The $\mathrm{x}$-rays are shown in the same scale as the damage maps. The bars across each image are the delamination width measured from the destructive inspections. "Upper" refers to delamination between the upper (impacted) aluminum layer and the prepreg and "lower" refers to delamination between the lower aluminum layer and the prepreg.

Both the simple-tiebreak interface and corrected-tiebreak interface simulations over-predicted the amount and extent of the delamination damage for the GLARE-3. In general the predicted delamination damage from the UMAT simulations agreed well with the damage patterns from the experiments.

The GLARE-4 damage patterns show that the delamination damage is more extensive in one direction than the other. This is a consequence of the 70/30 split between the fibres in the $0^{\circ}$ and $90^{\circ}$ directions. The same effect was noted in the $\mathrm{x}$-rays of the delaminated GLARE-4 specimens. This difference in the amount of fibres ultimately leads to fracture perpendicular to the weaker fibre direction when the impact load is high enough.

The predicted GLARE-5 damage geometries also agreed very well with the experiments. As shown in Figures 111 to 113, the shape of the predicted delamination is oval in shape. The same shape was observed in the impacted test coupons. 

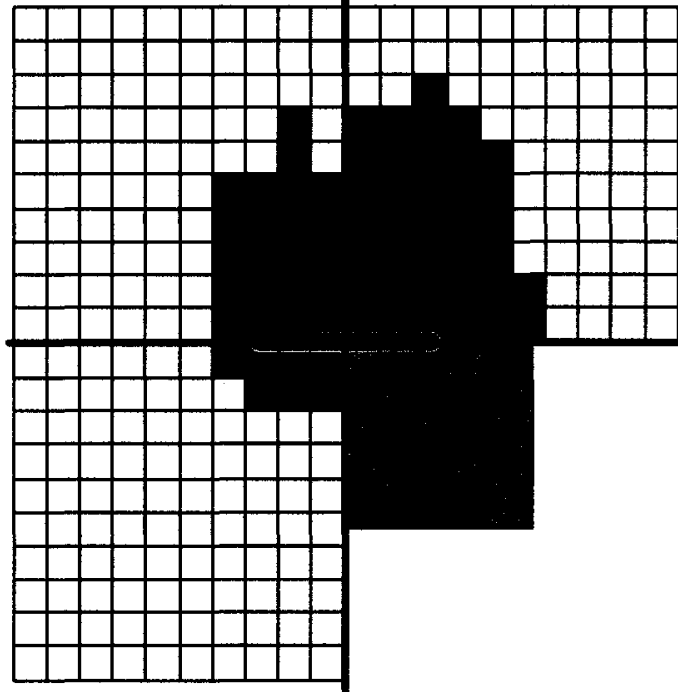

Upper and lower

Upper only

Lower only

Delamination width

from cross-section

GLARE-3 UMAT 25J GLARE-3 Experimental 25J

Figure 106: Damage maps of GLARE-3 impacted at $25 \mathrm{~J}$ from experiment, simple-tiebreak interface, corrected-tiebreak interface and UMAT simulations. [Note that the $x$-ray did not show any damage.]

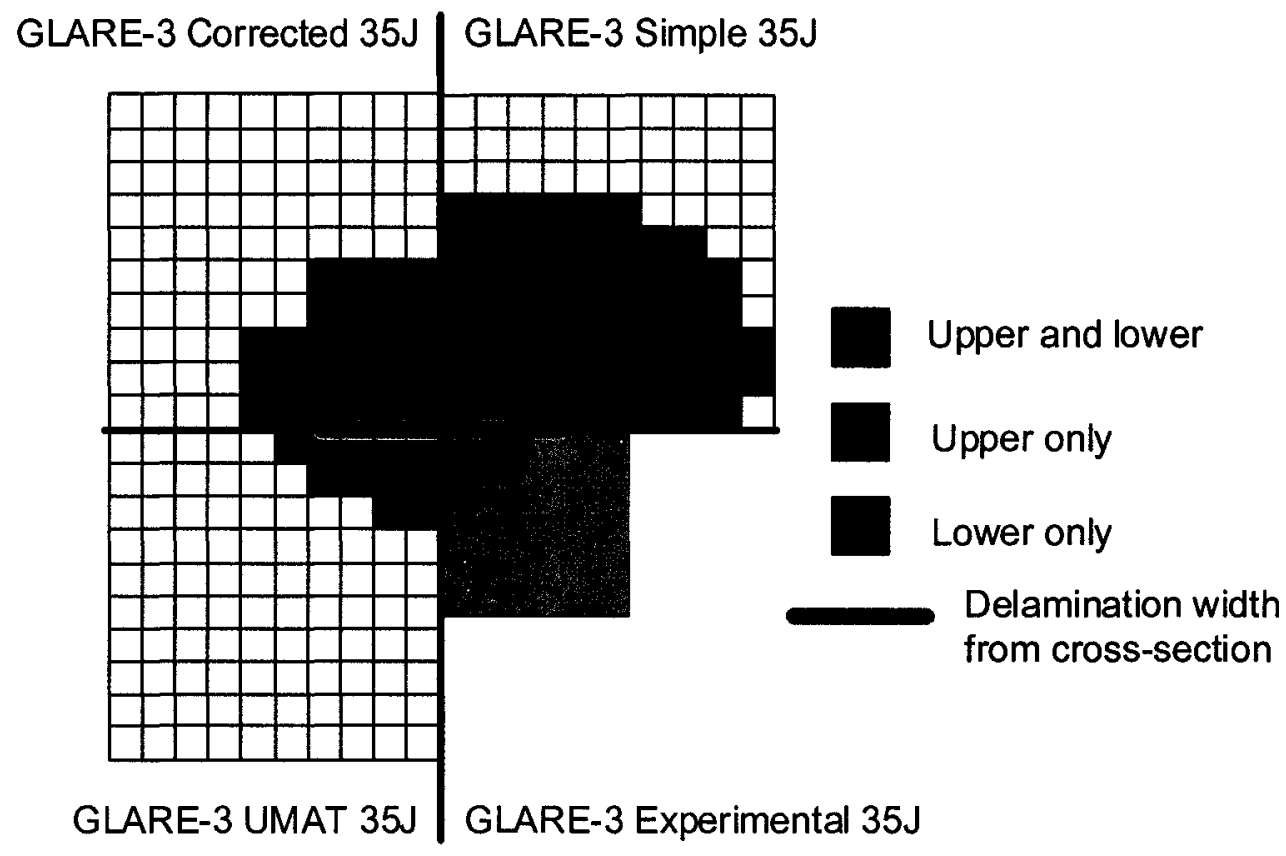

Figure 107: Damage maps of GLARE-3 impacted at $35 \mathrm{~J}$ from experiment, simple-tiebreak interface, corrected-tiebreak interface and UMAT simulations. 


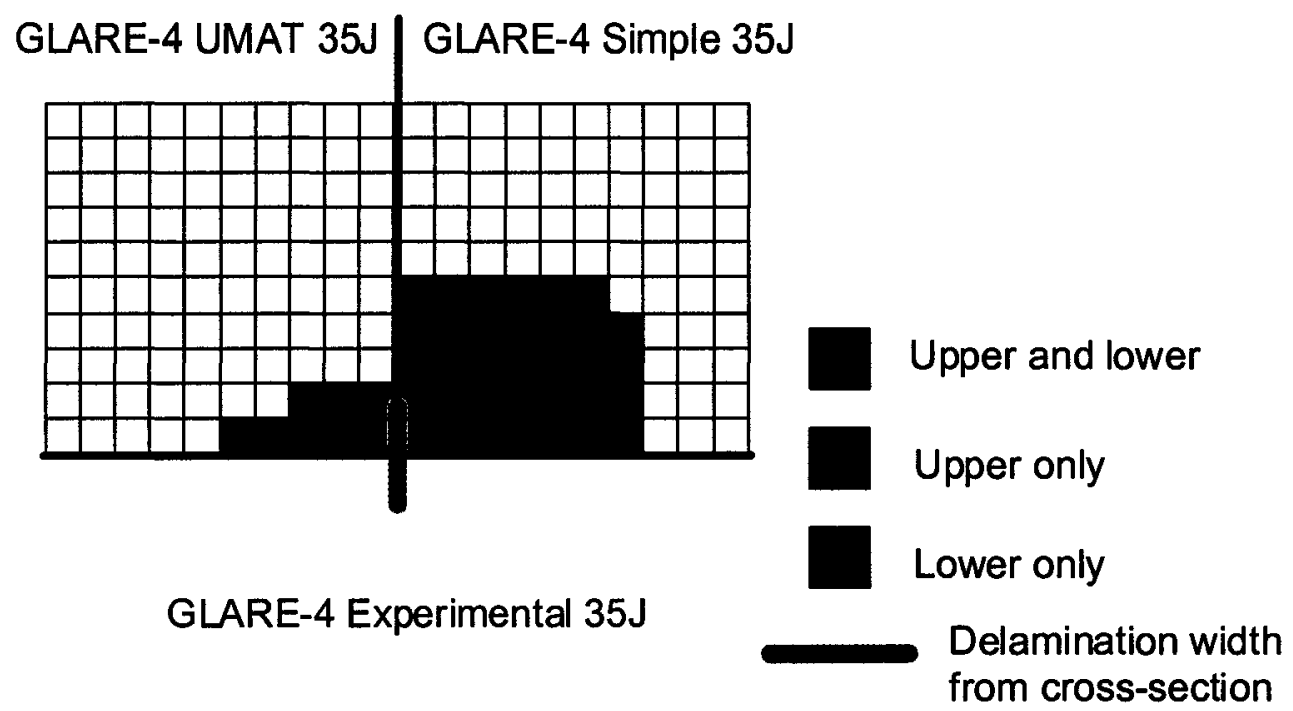

Figure 108: Damage maps of GLARE-4 impacted at $35 \mathrm{~J}$ from experiment, simple-tiebreak interface and UMAT simulations. [Note that no $x$-ray was available for this specimen.]

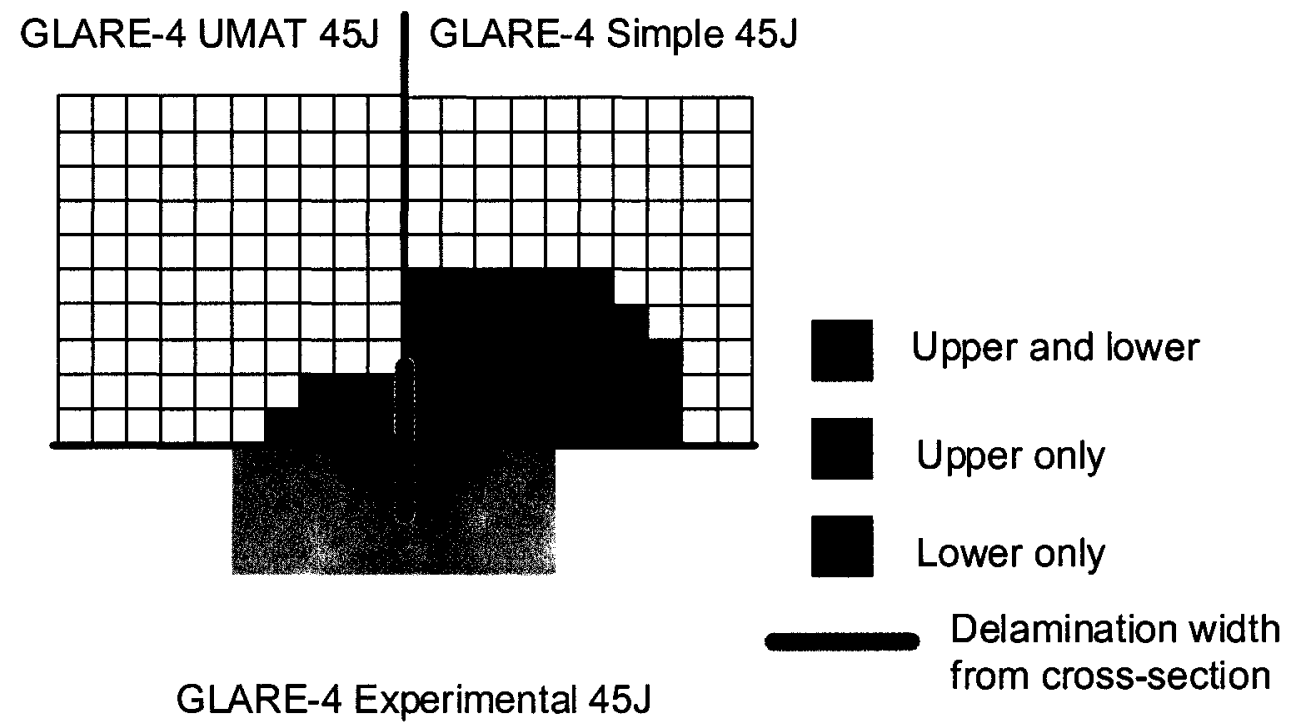

Figure 109: Damage maps of GLARE-4 impacted at $45 \mathrm{~J}$ from experiment, simple-tiebreak interface and UMAT simulations. 


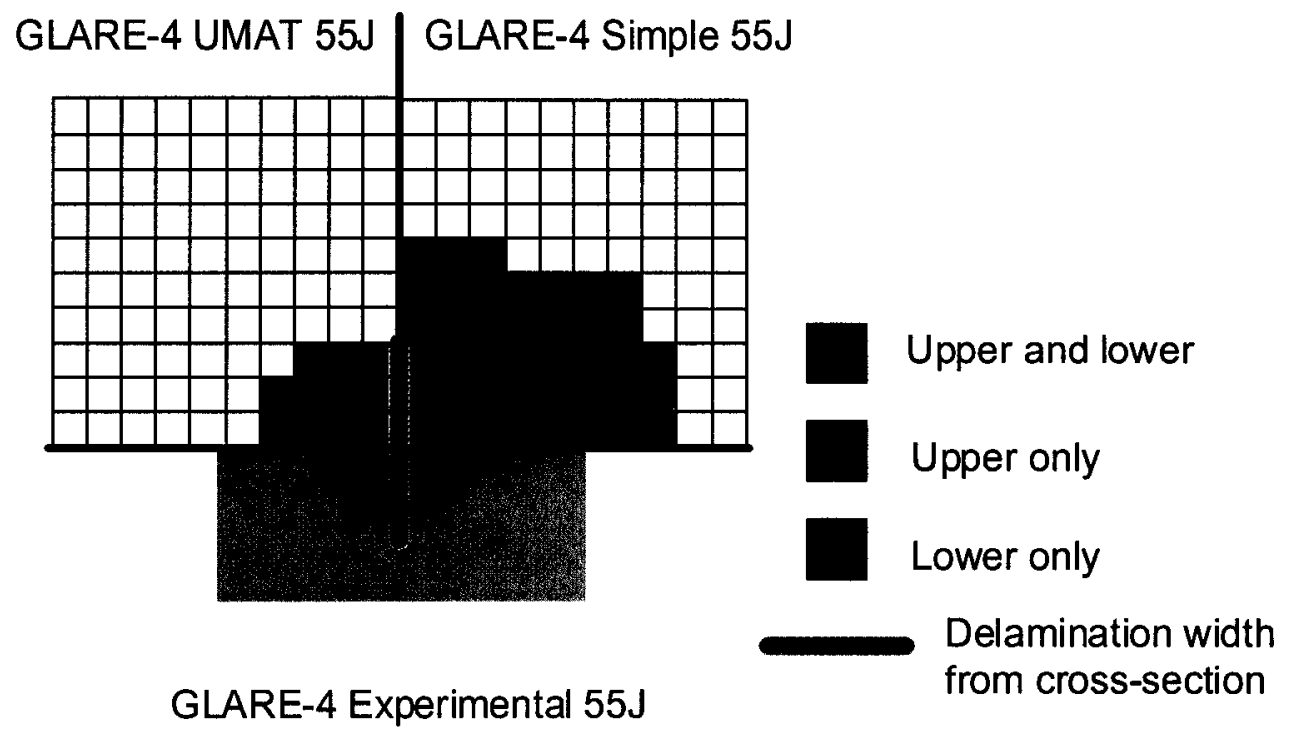

Figure 110: Damage maps of GLARE-4 impacted at $55 \mathrm{~J}$ from experiment, simple-tiebreak interface and UMAT simulations.

GLARE-5 UMAT 35J

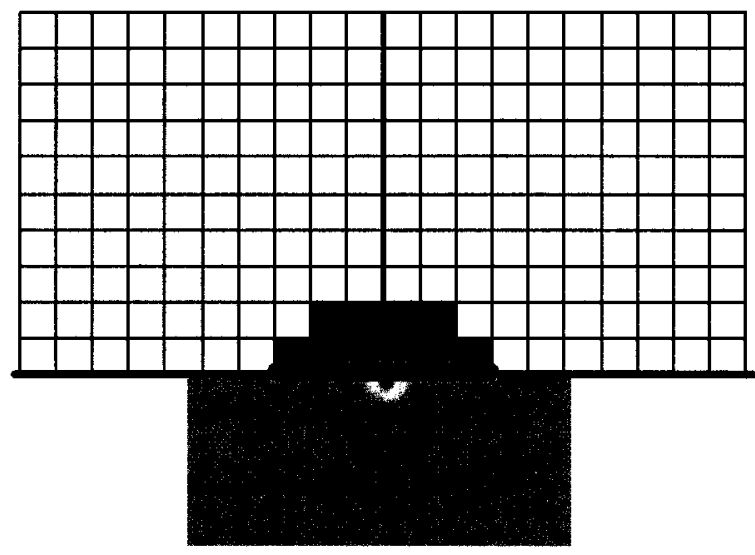

GLARE-5 Experimental 35J

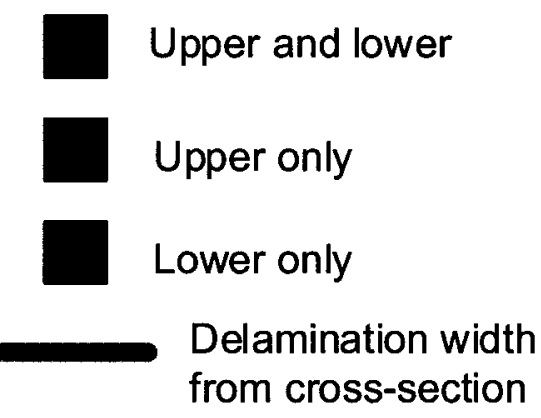

Figure 111: Damage maps of GLARE-5 impacted at $35 \mathrm{~J}$ for experiment and UMAT simulations. [Note that the $x$-ray did not show any damage.] 
GLARE-5 UMAT 45J

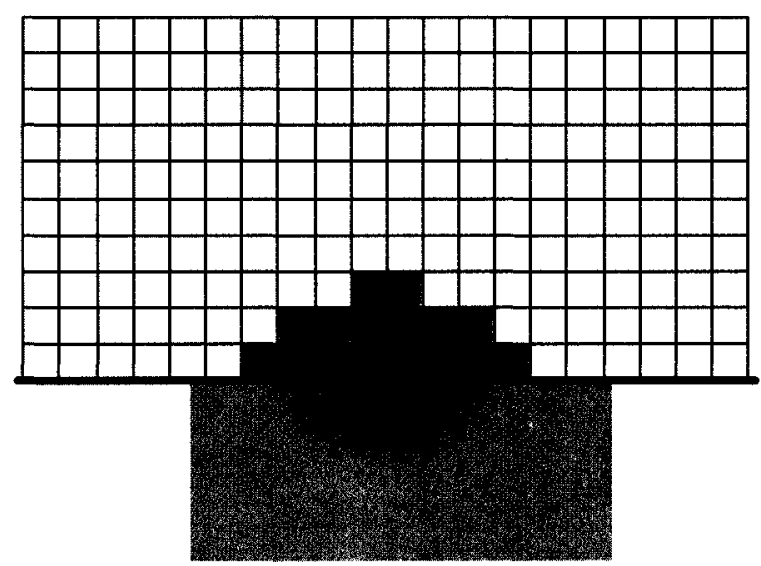

Upper and lower

GLARE-5 Experimental 45J

Upper only

Lower only

Delamination width from cross-section

Figure 112: Damage maps of GLARE-5 impacted at $45 \mathrm{~J}$ for experiment and UMAT simulations. [No destructive inspection was carried out for this sample.]

GLARE-5 UMAT 55J

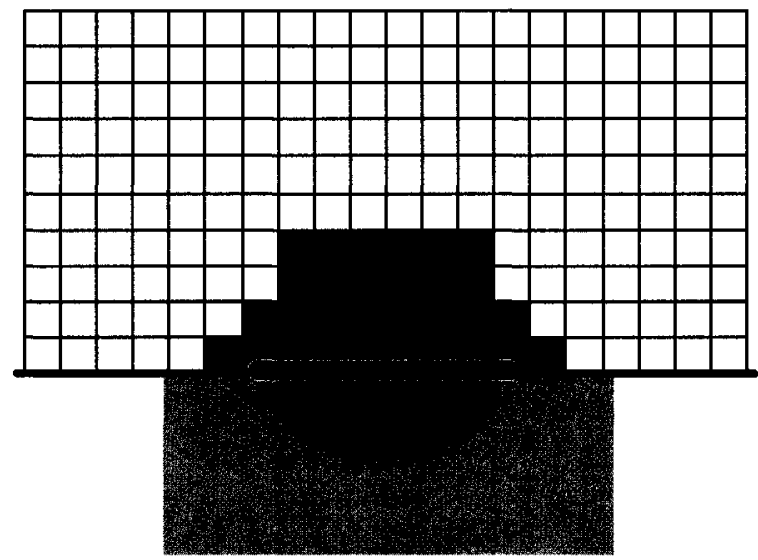

Upper and lower

GLARE-5 Experimental 55J
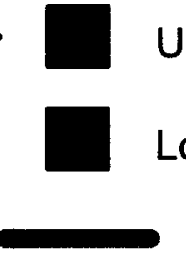

Upper only

Lower only

Delamination width from cross-section

Figure 113: Damage maps of GLARE-5 impacted at $55 \mathrm{~J}$ for experiment and UMAT simulations. 


\section{CHAPTER 8}

\section{SUMMARY AND DISCUSSION}

\section{OF RESULTS}

A modelling methodology to predict the formation of impact damage in FMLs was developed as part of this research. An extensive series of impact and characterization tests was carried out to support the development of the methodology. This methodology was used to study the impact response and resulting damage geometry of GLARE subjected to low-velocity impact. The damage modelling methodology can be developed further to predict failure of all layers of the FML. 


\subsection{Experimental Impact Testing}

The impact tests conducted as part of this work provided several insights into the development of impact-induced damage in FMLs. For example, damage was observed to vary with the applied impact energy as follows:

- plastically deformed dent with no delamination,

- formation of internal delaminations and matrix cracking, damage becomes visible in penetrant enhanced $\mathrm{x}$-rays, and

- puncture then occurs and the geometry of the puncture depends on the GLARE lay-up as discussed below.

It was found that, when puncture occurs in the GLARE-3 and GLARE-5 laminates, the impactor did not rebound and the panel absorbed all of the impact energy. However, there is a limit to the amount of energy that can be absorbed by the panel. Beyond this limit the impactor will pass through the panel, retaining a portion of its kinetic energy. This remaining energy is absorbed by the structure of the drop tower when the crosshead is arrested by large rubber stops. The GLARE-5 laminates absorbed less energy, on an areal density basis, than GLARE-3, GLARE-4 and 2024-T3 when subjected to low-velocity impact (Figure 18). In these laminates, a greater portion of the impact energy is converted to elastic potential energy and then returned to the impactor in these laminates. This is due to the larger amount of glass-fibres in the $0^{\circ}$ and $90^{\circ}$ directions of the impact-optimized GLARE-5 laminates compared to GLARE-3 and GLARE-4. 
The lay-up of the specimens strongly influenced the resulting damage geometry, as shown by the formation of a single crack in GLARE-4 (Figure 30). In these laminates, the crack was oriented parallel to the major fibre direction, which is also the loading axis for GLARE-4. Thus, in locations such as in fuselage or wing skins, impact-induced cracks in GLARE-4 would not grow if the load was parallel to the crack direction. In the majority of applications the applied loads on the laminate would not be uniaxial, but would have an off-axis resultant, and would cause some crack growth. For example, as was described by Laliberté et al. (2001a), impact-induced cracks oriented at $45^{\circ}$ to the loading axis were observed to grow under cyclic loading. In GLARE-3 and GLARE-5, multiple cracks formed as a result of impact (Figures 29 and 31). The damage in GLARE-3 and GLARE-5 did not change appreciably in size once the panel was punctured.

Since only GLARE-X-2/1 laminates were available for these tests, other types of GLARE should be subjected to impact loading in the future. In particular, thicker laminates should be tested to examine the influence of the number of layers on the formation of damage.

The circular impact specimen developed in this research project removed the corner deformations that were observed in rectangular specimens supported in the NASA fixture. The deformation and slippage resulting from the use of the circular fixture was limited to the periphery of the specimens, well away from the central dent. This distance between the fixture-induced deformation and the central dent increased with the use of the circular fixture compared to the square fixture (Figure 12). 
Overall, the impact test method used in this thesis was successful. It may be beneficial to make minor changes to improve the test set-up further. In particular, a sensor to measure the displacement of the panel as it is impacted could be incorporated into the drop tower. This could either be a contact device such as an LVDT or a noncontact sensor such a laser-based displacement measuring system. A high-speed camera could also provide qualitative data about the impact response of the panels.

To measure the difference between the impact force recorded by the load cell in the crosshead and the actual impact force on the panel a separate force measurement system is recommended. A series of four load cells could be used in the fixture support pillars to record this load. The difference between this measurement and the crosshead measurement would provide a measure of the system losses.

The non-destructive inspection method used to examine the impact GLARE panels accurately revealed the delamination in specimens impacted at energies greater than $35 \mathrm{~J}$. In the lower energy specimens, this technique did not expose the delamination damage. With some modifications to the technique, such as the use of multiple holes, it would be possible to fully infiltrate the damaged regions with penetrant. Although the destructive inspection technique accurately depicted the damage in the specimens, this method is impractical for application to actual aircraft. Additional technologies should be investigated for application to the non-destructive inspection of impacted FMLs. These could include contact c-scan ultrasonic probes and laser ultrasound.

It was noted that all of the specimens showed significant matrix cracking in the unpunctured panels. This form of damage contributed to the overall energy absorption of the impacted panels. Fibre fracture was only observed in panels that had been punctured. 


\subsection{Damage Modelling Procedure for FMLs}

In order to rapidly examine a large number of geometries, boundary conditions and lay-ups a damage modelling methodology was required for FMLs. Following a review of pertinent literature, a damage modelling methodology for FMLs was proposed to enable the prediction of the extent and type of damage in impacted FMLs. This methodology required four components. These consisted of an elastic-plastic aluminum model, a prepreg damage model, a delamination initiation model and a post-delamination growth model. These components were each developed and validated through simulations in LS-DYNA and experimental testing.

\subsubsection{Impact Damage Modelling of Aluminum}

The first component of the modelling methodology investigated was the elasticplastic model for the aluminum layers. The aluminum simulations showed that boundary conditions have an effect on the predicted response of the panels. Panels with a clamped boundary absorbed $1 \mathrm{~J}$ to $3 \mathrm{~J}$ less than those with simply-supported edges. The simplysupported panels deflected more than the clamped panels and thus absorbed more strain energy. In the experimental impact tests the boundary condition of the panels lies between the two extremes of clamped and simply-supported. There was evidence of slipping at the edges of the impacted panels. A model of the entire impact fixture could be employed to investigate this behaviour further.

The aluminum impact simulation results also showed sensitivity to the input material properties. A wide range of properties for aluminum 2024-T3 is available in 
literature and it was desired to study the effect of using different "acceptable" properties on the impact response of the panels. Yield stresses of $285 \mathrm{MPa}, 350 \mathrm{MPa}$ and $420 \mathrm{MPa}$ from the manufacturer, MIL-HDBK-5H (DoD, 1998) and ASM (1990) were used. In the panel with the yield stress of $285 \mathrm{MPa}$, a greater amount of plastic deformation occurred. This panel absorbed more impact energy through increased permanent deformation, as shown in Figure 63. The peak impact forces also decreased as shown in Figure 64. The impact results from the panels with the manufacturer's properties were closest to the experimental values. Therefore, the manufacturer's properties were used for the remaining simulations. Where possible, it is always best to have access to actual property data for the material under investigation.

In finite element analyses, studies of sensitivity of the solution to the mesh size are always recommended. In the present work, the mesh density did not influence the results of the aluminum simulations. However, the type of element did have an effect on some results. The predicted absorbed energy and peak impact force using thin-shell, thick-shell and solid-brick elements were in agreement. The predicted values of absorbed energy were consistently lower than the experimental values, by an average of $4 \mathrm{~J}$. The predicted peak impact force values were also higher than the experimental values by an average of $1.2 \mathrm{kN}$. The source of these two differences may be related to the lack of fixture losses in the model. With the current experimental set-up it was not possible to quantify these losses. A fully detailed impact fixture simulation would be required to investigate the effects of fixture losses and boundary conditions on the predicted impact response. 
It was noted that the solid-brick elements used resulted in predicted dent depths approximately $1.7 \mathrm{~mm}$ less than the thin- and thick-shell elements. The dimensions of the solid-brick elements in the simulated specimen could explain this effect. It has been indicated in the literature that these elements exhibit increased stiffness when they have high ratios of length to thickness (Hallquist, 1998). This would have produced higher peak impact forces for the solid-brick simulations as compared to the other element types, which was not the case. The formulation of the solid-brick elements is likely the source of this difference. It was decided to forgo an in-depth evaluation of the solid-brick element formulation.

\subsubsection{Delamination Growth Characterization}

As previously explained in Section 5.2.4, an attempt was made to use the DCB data from the tests conducted as part of this project in LS-DYNA. However, it was found that the load-displacement curves could not be converted to the required stressdisplacement curves. Therefore, a simple stress-displacement curve was used within LSDYNA instead.

The test method must be re-evaluated if it is to be used to develop a delamination correction for GLARE in the future. Direct measurement of the forces and displacements at the interface may be required. An extensometer attached directly to the specimen would improve the accuracy of the measurements recorded during the testing. A smaller specimen that has the delamination growing over a much shorter length may allow for the measurement of the fibre-bridging loads in the laminate. This specimen could be built on a scale similar to that of a single element in the finite element simulations to provide 
meaningful fibre-bridging loads. A pressure or a piezoelectric sensor could be used to monitor the stresses in the interface before and after delamination. Care would have to be taken to ensure that any in-situ sensor would not influence the formation of damage. It would be possible to use the measured stress-displacement data directly in LS-DYNA.

\subsubsection{Development of Prepreg Damage Model}

The development of a material model based upon the theories of Ladevèze and Le Dantec (1992) and its implementation in LS-DYNA, as described in Section 6.2, was successful.

The model was initially evaluated in Mathcad. For a carbon fibre prepreg, a shear strength of $82 \mathrm{MPa}$ was predicted versus an experimental value of $83 \mathrm{MPa}$ and a predicted value of $79 \mathrm{MPa}$ reported by Ladevèze and Le Dantec (1992). This demonstrated that the model could provide a reasonable prediction of the static strength of a composite coupon. The material model was then tested in LS-DYNA to eliminate the possibility of serendipitous agreement between the Mathcad results and the published values. The model was implemented as a user-defined subroutine, or "UMAT". A series of tensile coupon simulations of GLARE was carried out using this model. Numerically, the model functioned well, with no computational problems such as hourglassing.

The tensile coupon simulations showed that the model could predict the elastic response of the prepreg identically to the LS-DYNA orthotropic-elastic (type 2) material model. It was found that the simulations were sensitive to the mesh density. A lower mesh density caused sudden drops in the predicted stress-strain curve. There were fewer 
elements to accumulate damage. Thus, when a single element failed, a significant portion of the load-carrying capacity of the coupon was lost.

It was noted that the $0^{\circ}$ GLARE-3-3/2 coupons with the damage simulation in the UMAT activated were stiffer than the experimental results after yielding (Figure 83). The prepreg material model only accounts for stiffness loss perpendicular to the fibres. However, stiffness is lost in the experiments in the fibre direction, through matrix plasticity and some fibre fracture. Without accounting for these effects the model would behave slightly stiffer than the measured experimental response. The predicted stressstrain curves for the $15^{\circ}$ GLARE-3-3/2 coupons using a higher mesh density agreed very well with experimental data (Figure 84).

The earlier work of Sun and $\mathrm{Wu}$ (1992) successfully used quasi-static data for their impact simulations of ARALL. They demonstrated that it is possible to use this data in dynamic applications. Furthermore, Vlot (1993b) observed that under impact velocities of $10 \mathrm{~m} / \mathrm{s}$, it is possible to use quasi-static properties in analytical impact response predictions with GLARE laminates. The impact simulations presented in this thesis ranged in velocity from $2.7 \mathrm{~m} / \mathrm{s}$ to $4.4 \mathrm{~m} / \mathrm{s}$; therefore, the use of static coupon data to validate the model was considered appropriate. 


\subsection{Impact Modelling of GLARE}

The four components of the impact damage modelling methodology were assembled to study the response of GLARE laminates to low-velocity impacts. To summarize, the following configurations were used to study the effect of prepreg material model and interface type on the predicted impact response:

1. Elastic-plastic aluminum, elastic prepreg and tied-interface,

2. Elastic-plastic aluminum, elastic prepreg and uncorrected tiebreak-interface,

3. Elastic-plastic aluminum, elastic prepreg and corrected-tiebreak interface, and

4. Elastic-plastic aluminum, UMAT prepreg and simple-tiebreak interface.

\subsubsection{Mesh Sensitivity}

As for the aluminum simulations, mesh density study was conducted. The predicted dent depth for the lowest mesh density was lower than those of the higher mesh density simulations (Figure 90). At the same time, the damage geometry predicted with the lower mesh density did not agree with the experiments (Figure 91). These two effects were caused by the paucity of nodes connecting the interface together in the impacted region. It only took the release of two or three nodes to completely delaminate the impacted area. The medium- and high-mesh densities predicted very similar damage geometries in agreement with the experiments. However, the CPU time required for the medium-density mesh was $40 \%$ of that required for the high-density simulations. Consequently, the medium-mesh density was selected for the GLARE simulations. 


\subsubsection{Predicted Panel Response}

The type of interface between the constituent layers was varied to study the effect on the predicted response and damage geometry of the panels. Three types of interface were examined with the elastic prepreg. The UMAT simulations were carried out using only the simple-tiebreak interface. The percent differences between the predictions and experiments for GLARE-3 are summarized in Figures 114 to 116 . All of the results quoted in the following sections are from these graphs. The results from GLARE-3 are representative of the behaviour of the other laminates.

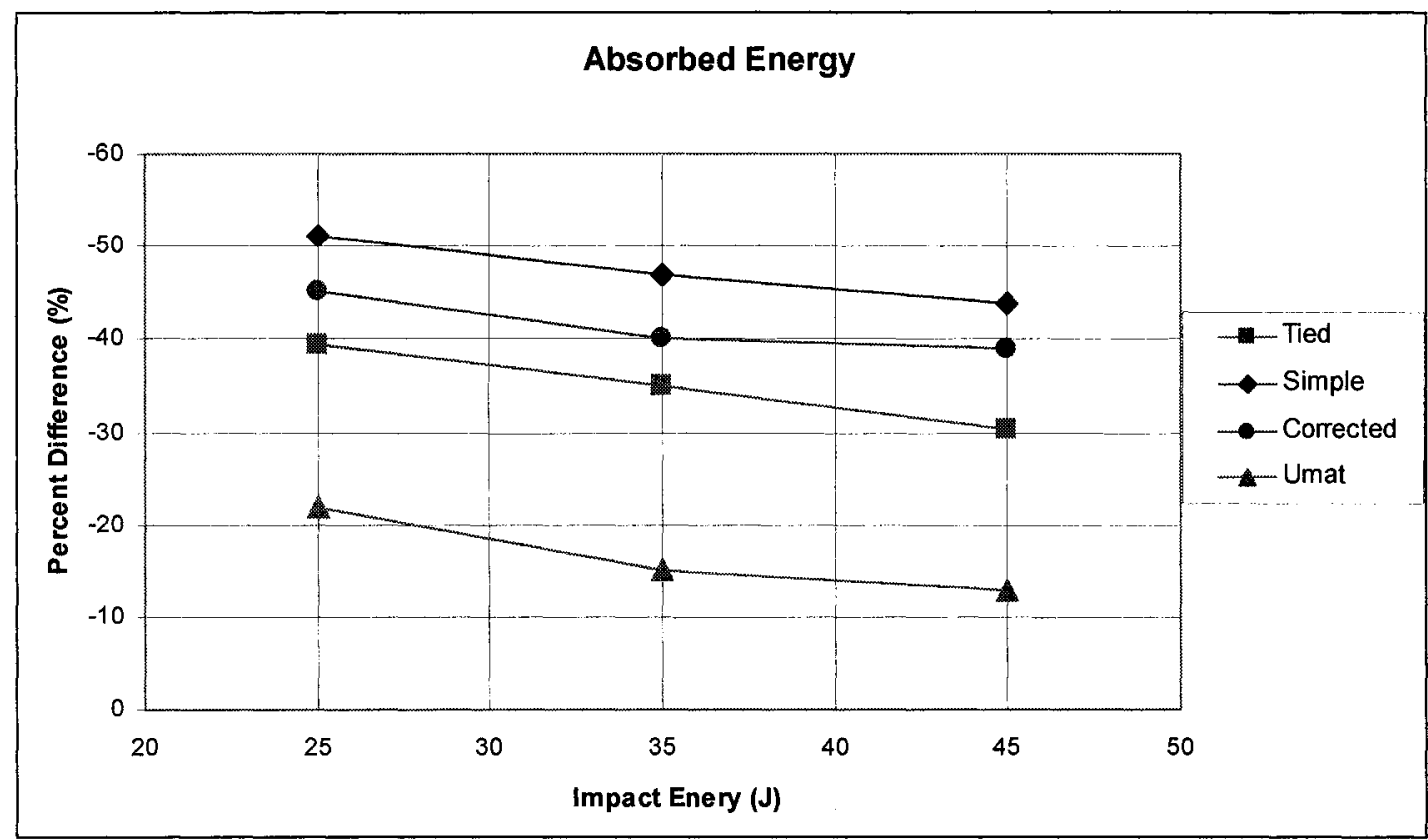

Figure 114: Percent difference between GLARE-3 absorbed energy predictions and experimental results. 


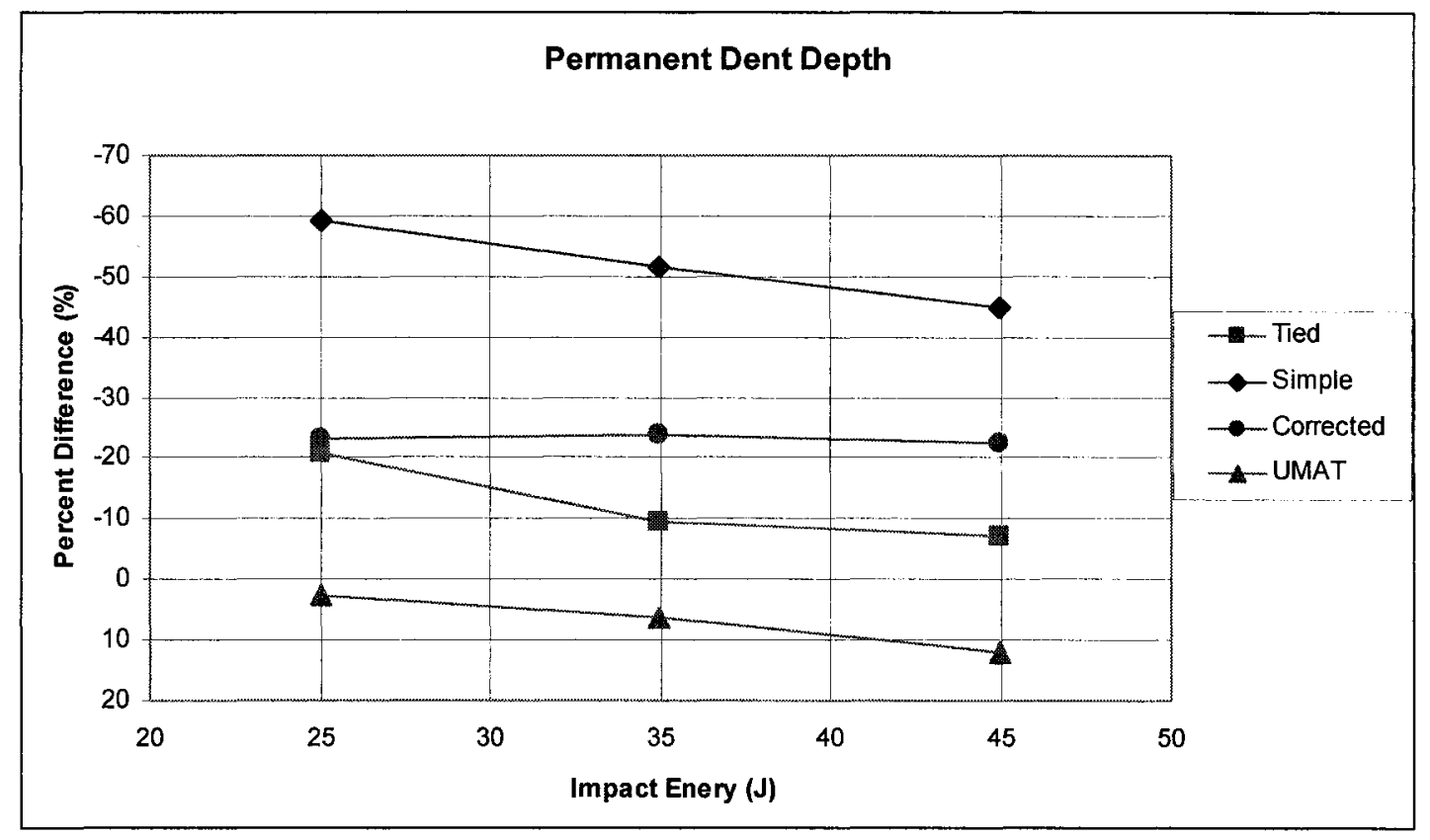

Figure 115: Percent difference between GLARE-3 permanent dent depth predictions and experimental results.

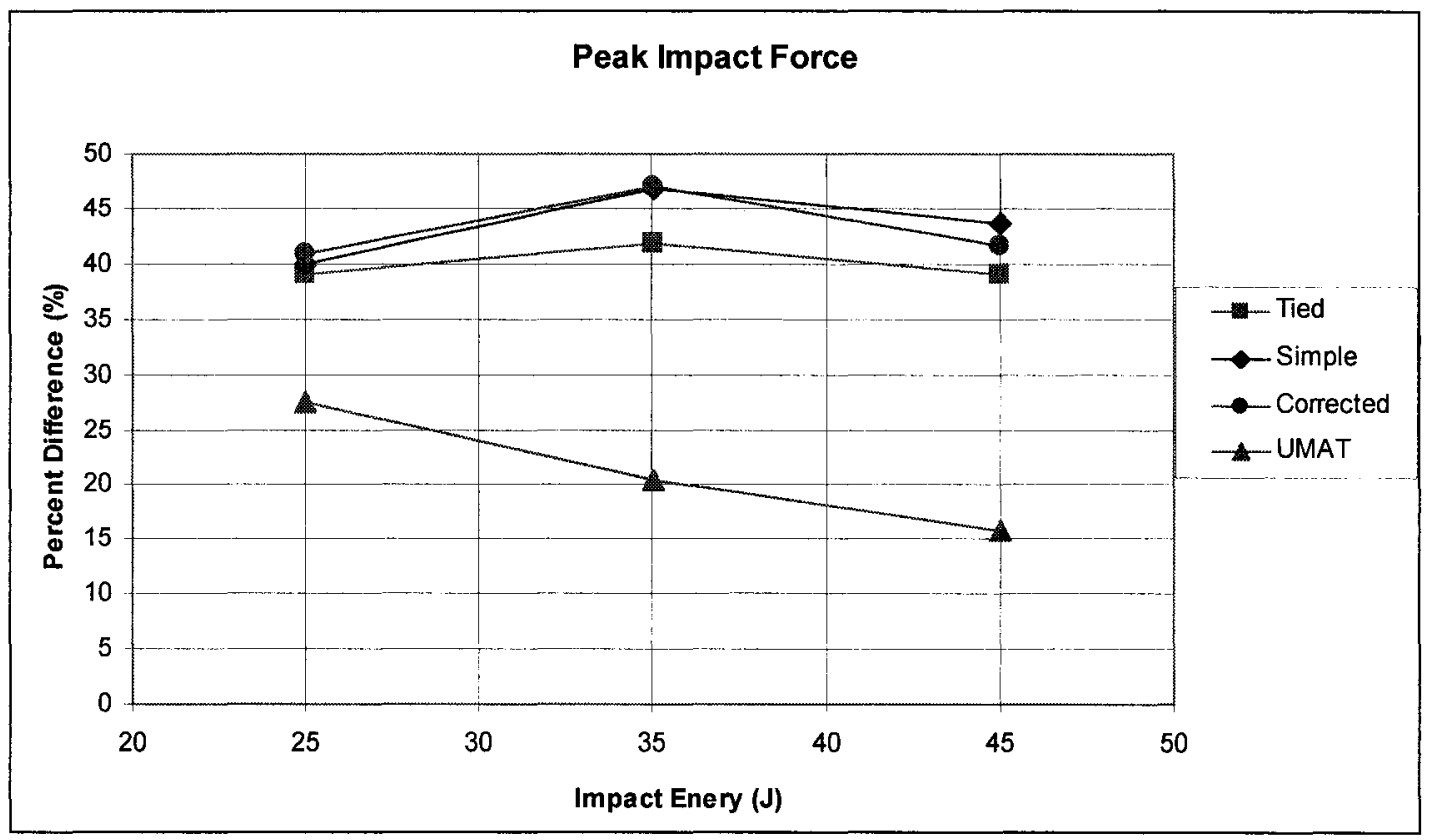

Figure 116: Percent difference between GLARE-3 peak impact force predictions and experimental results. 
The tied-interface fixed the layers together without failure. These simulations, with elastic prepreg, under-predicted the absorbed energy. The permanent dent depth predictions from these simulations were below the experimental values. The peak impact force was over-predicted. The same effects caused these differences. Both the plasticity of the aluminum and damage in the prepreg contributed to the permanent deformation of the experimentally impacted panels. Without the inclusion of damage in the prepreg layers, energy can only be absorbed through aluminum plastic deformation and stored elastic energy in all the layers. As well, with only the aluminum plasticity contributing to the permanent deformation, the dent depths would be lower. In fact, the stresses in the elastic prepreg layers actually try to push the aluminum layers upward but were prevented from doing so by the lack of delamination. Without the formation of damage, the effective stiffness of the tied-interface panels is higher than the experimental panels. This resulted in the higher peak impact force predictions.

The simple-tiebreak interface used the manufacturer's interface properties to predict failure. Simulations using this interface model, with the elastic prepreg, underpredicted the absorbed energy by the largest amount. The simple-tiebreak interface also resulted in lower dent depths than measured in the experiments. Finally, these simulations over-predicted the peak impact force. Unlike the tied-interface simulations, the formation of delamination damage was enabled in these simulations, permitting the rebound of the elastic prepreg layers. This released stored elastic energy in the prepreg layers, resulting in the lower absorbed energy prediction. The same action also pushed the aluminum layers upward, leading to considerably lower dent depths than the tiedinterface simulations. The peak impact force difference was slightly higher than that of 
the tied-interface simulations. The small difference indicates that the formation of delamination damage does not have a large influence on the impact forces in panels.

The corrected-tiebreak interface used a stress-displacement curve to represent the post-delamination behaviour of the interface. The corrected-tiebreak interface simulations, with elastic prepreg, under-predicted the absorbed energy. The permanent dent depth was also under-predicted and the impact force was over-predicted. The inclusion of the post-delamination load-displacement curve improved the absorbed energy prediction over the simple-tiebreak interface simulations by preventing the elastic prepreg layers from rebounding. The same effect lowered the dent depth prediction differences substantially. However, the peak impact force was still over-predicted with the corrected-tiebreak interface. This reinforces the fact that delamination damage does not have a great influence on the peak impact force in the impacted panels.

The UMAT simulations, using the simple-tiebreak interface, under-predicted the absorbed energy. However, the results were a substantial improvement over the previous simulations. As well, the permanent dent depth was over-predicted by a smaller percentage than the previous simulations. Finally, the peak impact force was overpredicted by a significantly lower amount than the simulations described above. Based on these results, it is postulated that the formation of intralaminar damage has a greater effect on the impact response of impacted FMLs than the formation of delamination damage. One can imagine that the combined surface area of the multitude of matrix cracks formed in the prepreg layers is much larger than the area formed by the delamination. Therefore, the energy released by formation of the matrix cracks would be proportionally larger than that released by the formation of delamination damage. 
Interestingly, the inclusion of damage in prepreg layers resulted in an over prediction rather than an under-prediction of the permanent dent depth. The reasons for this are not readily discernible.

Another effect to consider is the influence of the interlaminar and intralaminar damage on the in-plane and out-of-plane properties of the panels. Due to the large deformation caused by the impact event, it is the in-plane properties that have the greatest influence on the impact response. The formation of matrix cracks degrades these properties, reducing the stiffness of the panel and thus reducing the peak impact force. The formation of delamination damage has a greater effect on the out-of-plane properties of the laminate. Based on the impact force results, delamination damage does not have a significant influence the peak impact force. Although delamination damage does not have a significant effect on the impact response of the panels, the presence of delamination can affect the post-impact behaviour of the panels. For example, the compressive buckling sensitivity of GLARE could be increased by delamination.

While the inclusion of the UMAT improved the predicted response of the panels, there were still differences. Some of these differences could be accounted for by the lack of fixture effects in the simulations. No description of the effect of increasing the complexity of the model on the absorbed energy was found in literature. However, Majeed (1995) calculated a decrease of $22 \%$ in the peak impact force predicted for composite coupons by increasing the complexity of the model. This was achieved by switching from a rigid impactor and base to a complete model of the crosshead and base of the Dynatup rig with elastic properties. This is of a similar magnitude to the overprediction of the peak impact force by the UMAT simulations. It is also possible that the 
destructive inspections of the panels missed some fibre fractures. This damage mode would contribute to the overall energy absorption of the panels as well. A more detailed inspection of impacted panels may be required to eliminate this possibility.

\subsubsection{Predicted Damage Geometry}

The discussion on the damage geometry presented in the following paragraphs refers to Figures 104 to 113 . The tied-interface simulations, with the layers connected without failure, were not able to predict any delamination damage.

The delamination damage predicted with the simple-tiebreak interface and elastic prepreg was not like that observed during the experiments. In these simulations, the prepreg layers were elastic, therefore they rebounded after impact. This action pushed the top aluminum layer upward and reduced the depth of the resulting impact dent.

The use of the corrected-tiebreak interface, with the elastic prepreg, improved the predicted damage geometry of the panels and eliminated the rebound of the aluminum layers by introducing a restraining force between the aluminum and the prepreg layers. However, the extent of the delamination damage was still over-predicted by the corrected-tiebreak interface models compared with the experimental results.

The predicted damage geometries for the simulations with the UMAT prepreg model agreed well with the delamination damage measured following the experiments. The extent of the damage was accurately predicted as well as the general shape of the damage. For example, GLARE-4, which has a dominant fibre direction, was observed to have elliptical delamination damage following impact. The GLARE-4 simulations also predicted this damage geometry (Figures 108, 109 and 110). 
Based on the predicted delamination geometries, it is clear that the formation of delamination damage is inherently linked to the formation of damage in prepreg. With the elastic prepreg model, it was necessary to use interlaminar strength values significantly lower than those quoted by the manufacturer to achieve the formation of delamination. Even then, the predicted geometry did not resemble that of the experiments (Figure 105b). However, with the UMAT activated, the resulting geometry agreed very well with that of the experimentally impacted coupons. It was possible to achieve this result using the manufacturer's interlaminar strength properties.

\subsection{Limitations of the Modelling Procedure}

Although the simulations show good agreement with the experiments, the prepreg model does not account for plasticity effects in the matrix material. Literature sources indicate that these effects are negligible in FMLs (Wu and Sun, 1993). However, this effect should still be investigated in future implementations of the model. The model is also limited to simulations below the puncture threshold of GLARE since the prepreg material model does not include fibre fracture.

At present there is no direct way to visualize the level of damage in the elements of the simulation. This is a problem with LS-DYNA and the post-processing software. The only indicators of the level of damage in the prepreg are the stresses that are output by LS-DYNA. Despite the success of this project, much additional work is possible to improve the capability, accuracy and most importantly, applicability of this technique. 


\section{CHAPTER 9}

\section{CONCLUSIONS}

An extensive series of impact tests on GLARE laminates was completed in this research project. Also, a practical methodology for modelling the formation of impact damage in these laminates was developed and an initial evaluation was completed. This methodology is a synthesis of a CDM-based material model and a commercial finiteelement code. It was used to predict the response of GLARE panels subjected to impact loads. This methodology can form the foundation of an improved impact qualification procedure for FMLs. The designers of new FMLs will benefit from the availability of such a procedure. Conclusions drawn from this project are as follows:

1. GLARE-5, with a greater volume fraction of fibres, absorbs less impact energy on an areal density basis than aluminum 2024-T3, GLARE-3 or GLARE-4.

2. The orientation of a majority of the fibres in the $0^{\circ}$ direction results in the formation of a single crack in punctured GLARE-4 specimens. This crack grows with increasing impact energy. In the other GLARE variants the cracks developed in both fibre directions and did not increase in size with increasing impact energy. 
3. The DCB test methodology attempted in this work did not provide useful data for the prediction of the post-delamination response of the impacted panels. This issue remains to be resolved in future work.

4. The aluminum impact simulations demonstrated the applicability of LS-DYNA to the problem of simulating low-velocity impacts on FML panels.

5. The aluminum simulations were not sensitive to the mesh density, but showed a small sensitivity to the boundary conditions.

6. The CDM-based material model was used to accurately predict the static tensile response of composite and GLARE coupons compared with experimental data.

7. The UMAT, with the simple-tiebreak interface delamination model, showed some sensitivity to the mesh density. Lower mesh densities resulted in an overprediction of the extent of the delamination damage and an under-prediction of the dent depth. The mesh densities converged on a representative damage geometry.

8. As discussed, delamination does not appear to be the dominant damage mode in impacted GLARE laminates. Damage in the prepreg layers is a more influential damage mode and is responsible for the majority of the energy absorbed in lowvelocity impacts. Delamination damage may need to be considered when examining the post-impact properties of GLARE laminates.

9. The geometry of delamination damage in the impacted panels was accurately predicted using the CDM-based damage model. The geometry and the size of the predicted delaminated regions agreed with the experiments. As was observed in the experiments, the predicted damage was affected by the lay-up of the coupons. 


\section{CHAPTER 10}

\section{FUTURE WORK}

The successful implementation of a damage modelling methodology for FMLs is the first step in a process that will lead to a deeper understanding of the fundamental damage processes in these materials. Monolithic alloys have been employed for centuries in engineering structures and even relatively modern composite materials have been in widespread use for decades. Fibre-metal-laminates are a comparatively new material. Thus, there remains a large amount of work to develop a complete set of tools to analyze all of the damage modes in these materials. The following sections describe some suggested topics for follow-on work in the area of FML damage modelling including possible improvements to the existing methodology and additional applications. 


\subsection{Experimental Testing}

There are numerous tests that should be conducted on FMLs to improve the understanding of their response to damage. Vlot (1993) described a method for impact testing ARALL under an applied tensile load. Similarly, GLARE laminates should be impacted under applied biaxial and uniaxial loads. This boundary condition better represents the loads that FMLs would experience in certain locations on an aircraft. These tests would be supported by an expanded series of simulations using the damage modelling methodology with applied boundary loads.

New FML material systems are being developed with different types of prepregs and alloys. For example, TiGr laminates are being considered as a possible material for application on the F-35 Joint Strike Fighter. Additional characterization tests could be conducted using the method of Ladevèze and Le Dantec (1992) on these new constituent materials. These materials would also include the most recent GLARE prepreg materials, such as Cytec FM-94. Fibre-metal-laminates produced with woven prepregs should also be characterized. This would support further development of the CDM-based prepreg model and allow its application to the new laminates. The modelling methodology could also be applied in simulations with more complicated loading conditions including shear, compression and bending. 


\subsection{Full Failure Prediction of FMLs}

The modelling methodology could be expanded to include the failure of the fibres and the aluminum layers. This would allow for the prediction of fracture and penetration in the impacted laminates. One possible approach for modelling the failure of the prepreg in the fibre-direction is to assume that the prepreg is elastic until the ultimate strength in that direction is reached. The element would then no longer be able to carry loads in that direction. A similar approach can be used to predict failure in the aluminum layers based on strain to failure. There are existing material models for metals in LS-DYNA that could be used for this application. The CDM-based prepreg material model would have to be updated with additional code to include failure in the fibre-direction. 


\subsection{Improved Delamination Modelling}

An improved method for predicting the failure of the interface between the layers is required. The CDM-based delamination modelling approaches described in Section 6.1 would be appropriate for implementation in a finite element framework (Allix et al., 1991, Allix and Ladevèze, 1992, Daudeville and Ladevèze, 1993, Daudeville et al., 1995, Allix and Ladevèze 1995 and Allix et al., 1998). The current model used for predicting the interface failure in LS-DYNA does not incorporate degradation of the interface. The existing DCB data or additional delamination testing could support this development. Since it was noted that the formation of delamination damage did not significantly influence the impact response of the panels, it is not necessary to employ a more sophisticated delamination model for thin laminates. The current methodology does provide an accurate prediction of the delamination size. Predicting the size of the delamination damage is important when considering the post-impact response of the laminates. However, in thicker GLARE laminates, where the out-of-plane properties would exert more influence, an improved delamination model should still be investigated. 


\subsection{Miscellaneous Extensions and Additions to the Modelling Methodology}

To further extend the capabilities of the modelling methodology put forward in this thesis, the CDM-based damage model should be implemented for solid-brick elements in LS-DYNA. This would allow the model to be applied to more complex problems. Additionally the strain-rate sensitivity of the fibreglass prepreg should be investigated and, if required for specific problems, incorporated into the prepreg material model. The modelling methodology could also be applied in implicit finite element codes such as ABAQUS or the implicit part of LS-DYNA. This would improve its applicability to static loading simulations.

Full simulations of the entire impact fixture should be conducted to further examine the effects of slippage between the clamping plates and the impacted specimens. These simulations could also be used to investigate losses due to compliance in the impact fixture and crosshead. 


\subsection{Additional Applications}

There are numerous additional applications of the FML damage modelling methodology that could be investigated as listed below.

- Application of the model to the problem of residual stress development on the aluminum layers following impact. Presently the stress-state in these layers following impact is the subject of debate. Knowledge of the stress-state would aid in the prediction of post-impact fatigue behaviour.

- Application of the model to the problem of forming of GLARE laminates. Although the current trend is towards the preparation of built-up components from GLARE, forming may still be required to minimize costs or to produce simple parts.

- The National Research Council, Bombardier Aerospace and Carleton University are currently investigating the problem of buckling and post-buckling of stiffened GLARE panels and cylinders. The finite element models use a single layer of shells with the material properties "smeared" across the laminate. A multi-layered approach using the CDM-based prepreg model could improve the accuracy of these simulations.

- A parametric study of different impactors and specimen configurations should be conducted in LS-DYNA. The model could also be scaled up to simulate actual aircraft components such as the leading edge of a wing. 


\section{REFERENCES}

Allix, O., Daudeville, L. and Ladevèze, P. "Delamination and Damage Mechanics," $4^{\text {th }}$ International Mecamet Seminar on Mechanics and Mechanisms of Damage in Composites, 1991, pp. 143-157.

Allix, O. and Ladevèze, P. "Interlaminar Interface Modelling for the Prediction of Delamination," Composite Structures, 1992, v. 22, pp. 235-242.

Allix, O. and Ladevèze, P. "Damage Analysis of Interlaminar Fracture Specimens," Composite Structures, 1995, v. 31, pp. 61-74.

Allix, O., Lévêque, D. and Perret, L. "Identification and Forecast of Delamination in Composite Laminates by an Interlaminar Interface Model," Composites Science and Technology, 1998, v. 58, pp. 671-678.

Armstrong, C. and Fawaz, Z. "Infrared Thermography as a Rapid NDI Tool for Advanced Composite Materials," $3^{\text {rd }}$ Canadian-International Composites Conference (CANCOM-01), 2001, Montreal, Canada.

ASM, “ASM Handbook,” ASM International Handbook Committee, 1990, $10^{\text {th }}$ ed.

Asundi, A. and Choi, Alta Y.N. "Fiber Metal Laminates: An Advanced Material for Future Aircraft," Journal of Materials Processing Technology, v. 63, 1997, pp. 386-394.

Awerbuch, J., "Experimental Investigation on Delamination Progression in ARALL Laminates," Alcoa report, 1987.

Belytschko, T., Lin, J. and Tsay, C.-S. "Explicit Algorithms for the Nonlinear Dynamics of Shells," Computer Methods in Applied Mechanics and Engineering, 1984, v. 42, pp. $225-251$. 
Bijlmer, P.F.A. "Fracture Toughness of Multiply Layer Adhesive Bonded Aluminum Alloy Sheet," International Congress of the Aeronautical Sciences, 1978, Lisbon, 544554.

Boeing Specification Support Standard 7260, "Advanced Composite Compression Tests," 1988, Boeing Company, Seattle, WA.

Brewer, J.C. and Lagace, P.A. "Quadratic Stress Criterion for Initiation of Delamination," Journal of Composite Materials, 1988, v. 22, pp. 1141-1155.

Bucci, R.J., Mueller, L.N., Vogelsang, L.B. and Gunnink, J.W. "ARALL® Laminates," Treatise on Materials Science and Technology, v. 31, 1989.

Chaboche, J.L. "Continuum Damage Mechanics: Part 1 - General Concepts," Journal of Applied Mechanics, 1988, v. 55, pp. 59-64.

Chang, F.-K. and Springer, G.S. "The Strengths of Fibre Reinforced Composite Bends," Journal of Composite Materials, v. 21, 1987, pp. 30-45.

Choi, H.Y. and Chang, F.-K. "A Model for Predicting Damage in Graphite/Epoxy Laminated Composites Resulting from Low-Velocity Point Impact," Journal of Composite Materials, 1992, v. 26, n. 14, pp. 2134-2169.

Coleman, B. and Gurtin, M. "Thermodynamics with Internal Variables," Journal of Chemistry and Physics, 1967, v. 47, pp. 597-613.

Daudeville, L. and Ladevèze, "A Damage Mechanics Tool for Laminate Delamination," Composite Structures, 1993, v. 25, pp. 547-555.

Daudeville, L., Allix, O. and Ladevèze, P. "Delamination Analysis by Damage Mechanics: Some Applications," Composites Engineering, 1995, v. 5, pp. 17-24. 
Delfosse, D. and Poursartip, A. "Energy-Based Approach to Impact Damage in CFRP Laminates," Composites Part A, 1997, v. 28A, pp. 647-655.

Department of Defense (DoD), "Metallic Materials and Elements for Aerospace Vehicle Structures," MIL-HDBK-5H, 1998.

Fahr, A., Chapman, C.E., Forsyth, D.S., Laliberté, J.F. and Poon, C. "Non-Destructive Evaluation Methods for Damage Assessment in Fibre Metal Laminates," International Symposium on Polymer Composites Science and Technology, SPE Topical Conference, 1999, Quebec City, Canada.

Fahr, A., Chapman, C. E., Laliberté, J. and Poon, C., "Non-Destructive Evaluation Methods for Damage Assessment in Fiber-Metal Laminates," Polymer Composites, 2000, v. 21, n. 4 .

Flanagan, D.P. and Belytschko, T., "A Uniform Strain Hexadron and Quadrilateral and Orthogonal Hourglass Control," International Journal of Numerical Methods in Engineering, 1981, v. 17, pp. 679-706.

Freischmidt, G., Coutts, R.S.P. and Janardhana, M.N. "Aluminum/Lithium Alloy-Carbon Fibre/Epoxy Laminated hybrid Composite Material: Part I Preliminary Results." Journal of Materials Science Letters, 1994, v. 13, n. 14, pp. 1027-1032.

Germain, P., Ngyuen, Q. and Suquet, P. "Continuum Thermodynamics," Journal of Applied Mechanics, 1983, v. 50, pp. 1010-1020.

Gregory, M.A. and Roebroeks, G.H.J.J. "Fibre-metal-laminates: A Solution to Weight, Strength and Fatigue Problems." $30^{\text {th }}$ Annual CIM Conference of Metallurgists, 1991 Ottawa, Canada, pp. 43-56.

Guo, Y.J., Wu, X.R. and Zhang, Z.L. "Characterisation of Delamination Growth Behaviour of Hybrid Bonded Laminates," Fatigue and Fracture of Engineering Materials and Structures, 1997, v. 20, n. 12, pp. 1699-1708. 
Hallquist, J. LS-DYNA Theoretical Manual, 1998. Livermore Software Technology Corporation (LSTC).

Hallquist, J.O., LS-DYNA Users Manual, 1997, LSTC Report number 1082.

Hashagen, F., de Borst, R. and de Vries, T. "Delamination Behavior of Spliced Fiber Metal Laminates Part 2. Numerical Investigation," Composite Structures, 1999, v. 46, pp. 147-162.

Hashagen, F., Schellekens, J.C.J., Borst, R. de and Parisch, H., Finite Element Procedure for Modelling Fibre Metal Laminates, Composite Structures, 1995, v. 32, n. 1, pp. 255 264.

Hashemi, S., Kinloch, A., Williams, J., Corrections Needed in Double-Cantilever Beam Tests for Assessing the Interlaminar Failure of Fibre-Composites, Journal of Material Science Letters, 1989, v.8, pp. 125-129.

Hashin, Z. "Failure Criteria for Unidirectional Fiber Composites," Journal of Applied Mechanics, 1980, v. 47, pp. 329-334.

Hertzberg, R.W., Deformation and Fracture Mechanics of Engineering Materials, 1996, John Wiley and Sons, Inc, New York.

Hoof, J. van, "Modeling of Impact Induced Delamination in Composite Materials," $\mathrm{PhD}$ Thesis, Ottawa-Carleton Institute for Mechanical and Aerospace Engineering, 1999.

Hoogsteden, W.P. "Compression After Impact Behaviour of ARALL®-1 Laminates." Wright Laboratory, Wright-Patterson AFB, 1992.

Hult, J. "Introduction and General Overview," Continuum Damage Mechanics: Theory and Applications, ed. D. Krajcinovic and J. Lemaitre, 1987. 
Hughes, T.J.R., Taylor, R.L., Sackman, J.L., Curnier, A.C. and Kanoknukulchai, W. "A Finite Element Method for a Class of Contact-Impact Problems," Journal of Computer Methods in Applied Mechanics and Engineering, 1976, v. 8, pp. 249-276.

Hung, K.-S. "Numerical Studies on the Delamination Mechanism in Laminated Composites under Impact Loading," $10^{\text {th }}$ International Conference on Composite Materials (ICCM-10), Whistler, Canada, 1995, v. 5, pp. 623-630.

Hurez, A., Roelandt, J.M. and Abisor, A., "Numerical Modelling of Elastic-Plastic Behaviour Coupled with Damage in Metal/Composite Laminates. Application to ARALL and GLARE," ICCM-9, 1993, Madrid, Spain.

Johnson, W.J. "Damage Tolerance Evaluation of Adhesively Laminated Titanium." Journal of Engineering Materials and Technology, 1983, v. 105, pp. 182-187.

Johnson, W.S. "Impact and Residual Fatigue Behaviour of ARALL and AS6/5245 Composite Materials." NASA Technical Memorandum 89013, October 1986, NASA Langley Research Center.

Jodoin, A., Shi, G., Poon, C., and Straznicky, P., "Experimental Analysis of Diagonal Tension in GLARE," 48 $8^{\text {th }}$ Annual Conference of Canadian Aeronautical and Space Institute, April 29 - May 2, 2001a, Toronto, Canada.

Jodoin, A., Poon, C., Straznicky, P. V., Shi, G., and Kok, L., "Diagonal Tension Behaviour in GLARE," $8^{\text {th }}$ International Conference on Composites Engineering (ICCE8), August 5-11, 2001b, Tenerife, Spain.

Jodoin, A. Poon, C., Straznicky, P.V., Shi, G. and L. Kok, "Diagonal Tension Behavior in GLARE" to be presented at the International Council of Aeronautical Sciences Congress, Toronto, Sept. 8-13, 2002.

Jones, R. M. Mechanics of Composite Materials, 1975, Scripta Book Company. 
Kachanov, L.M. "On Creep Rupture Time," Izv. Acad. Nauk SSSR, Otd. Techn. Nauk, n. 8, 1958, pp. 26-31 [in Russian].

Kaufman, J.G. "Fracture Toughness of 7075-T6 and -T651 Sheet, Plate and MultiLayered Adhesive-Bonded Panels." Journal of Basic Engineering, 1967, v. 89, pp. 503507.

Kim, R.Y., Soni, S.R., "Delamination of Composite Laminates Stimulates by Interlaminar Shear," Composite Materials, 1986, pp. 286-307.

Krajcinovic, D. "Constitutive Equation for Damaging Materials," Journal of Applied Mechanics, 1983, v. 50, pp. 355-360.

Ladevèze, P. and Le Dantec, E., "Damage Modelling of the Elementary Ply for Laminated Composites," Composites Science and Technology, 1992, v. 43, pp. 257-267.

Laliberté, J. and Poon, C. "Durability of Hybrid Fibre Metal Laminates," extended abstract in International Committee on Aeronautical Fatigue (ICAF) Summary, 1999a.

Laliberté, J., Poon, C. and Straznicky, P.V. "Application of Fibre-Metal Laminates in Airframe Structures," International Symposium on Polymer Composites Science and Technology, SPE Topical Conference, 1999b, Quebec City, Canada.

Laliberté, J., Poon, C. and Straznicky, P.V. "Low-Velocity Impact Damage in GLARE Fibre-Metal Laminates," ICCM-12, 1999c, Paris, France.

Laliberté, J., Poon, C. and Straznicky, P.V., "Impact Damage Tolerance in Composites and Fibre Metal Laminates," Canada-Taiwan Symposium on Aircraft Materials Testing, Maintenance and Repair, 1999d, Ottawa, Canada.

Laliberté, J., Poon, C. and Straznicky, P.V., "Durability Testing of Fibre-MetalLaminates," IAR Laboratory Report Number LTR-ST-2231, 1999e, National Research Council Canada. 
Laliberté, J., Poon, C., Straznicky, P.V. and Fahr, A. "Applications of Fibre-Metal Laminates in Airframe Structures," Polymer Composites, 2000a, v. 21, n. 4.

Laliberté, J., Poon, C., Straznicky, P.V. and Fahr, A. "Post-Impact Fatigue in FiberMetal Laminates," $2^{\text {nd }}$ International Conference on the Fatigue of Composites (ICFC-2), 2000b, Williamsburg, USA.

Laliberté, J., Poon, C. Straznicky, P.V., Fawaz. Z. and McCuaig, K. "Post-Impact Biaxial Fatigue Crack Growth in GLARE," $21^{s t}$ ICAF Symposium, 2001a, Toulouse, France.

Laliberté, J., Straznicky, P.V. and Poon, C. "Post Impact Shear Behaviour in GLARE," CANCOM-01, 2001b, Montreal, Canada.

Laliberté, J., Straznicky, P.V. and Poon, C. "Mode-I Delamination in Cross-Ply FibreMetal Laminates," $10^{\text {th }}$ International Congress of Fracture (ICF-10), 2001c, Honolulu, USA.

Laliberté, J., McCuaig, K., Poon, C. and Straznicky, P.V. "Post-Impact, Off-Axis Fatigue of Glare-4 3/2 Variant and Aluminum," IAR-SMPL, Laboratory Memorandum, April 2002a [under review].

Laliberté, J., Straznicky, P.V. and Poon, C. "Numerical Modelling of Low-Velocity Impact Damage in Fibre-Metal Laminates," 2002 ICAS Congress, Toronto, Canada, September 2002b [accepted as a poster paper].

Lemaitre, J. "A Course on Damage Mechanics," 1992, Springer-Verlag, Amsterdam.

Lemaitre, J. "Formulation and Identification of Damage Kinetic Constitutive Equations," Continuum Damage Mechanics: Theory and Applications, ed.. D. Krajcinovic and J. Lemaitre, 1987.

Li, E. and Johnson, W.S. "An Investigation into the Fatigue of a Hybrid Titanium Composite Laminate," Journal of Composites Technology and Research, 1998, v. 20. 
Li, S.H, Zhou, B.L., Tang, Z.T. and Zeng, Q.Y. "Reformed Bamboo and Reformed Bamboo/Aluminum Composite: Part II Impact Properties." Journal of Material Science Letters, 1996, v. 15, n. 2, pp. 129-131.

Liaw, B.M., Liu, Y.X. and Villars, E.A., "Impact Damage Mechanisms in Fiber-Metal Laminates," SEM Annual Conference, 2001, Portland, USA.

Lin, C.T. and Kao, P.W. "Delamination Growth and its Effect on Crack Propagation in Carbon Fibre Reinforced Aluminum Laminates Under Fatigue Loading." Acta Materialia, 1996, v. 44, n. 3, pp. 1181-1188.

Majeed, O., Worswick, M.J., Straznicky, P.V. and Poon, C. "Numerical Modelling of Transverse Impact on Composite Coupons," Canadian Aeronautics and Space Journal, 1994, v. 40, n. 3, pp. 99-107.

Majeed, O. "Numerical Modelling of Transverse Impact on Composite Coupons," M.Eng Thesis, 1995, Carleton University.

Marissen, R. and Vogelsang, L.B. 'Development of a New Hybrid Material: 'ARALL,' Intercon. SAMPE Meeting, 1981, Cannes, France.

Mattousch, A. "Test Procedures for Fibre-metal-laminates." SLC Report Number TD-R93-003, 1993, Structural Laminates Corporation.

Matzenmiller, A., Lubliner, J. and Taylor, R. "A Constitutive Model for Anisotropic Damage in Fiber-Composites," Mechanics of Materials, 1995, v. 20, pp. 125-152.

Maugin, G.A. The Thermomechanics of Plasticity and Fracture, 1992, Cambridge University Press.

NASA Reference Publication 1092, "Standard Tests for Toughened Resin Composites," 1983, ACEE Composites Project Office, Langley Research Center, Hampton, VA, USA. 
Poon, C. and Benak, T. "Instrumented Drop Weight Testing Facility at the National Aeronautical Establishment," IAR Laboratory Report Number LTR-ST-1605, 1986, National Research Council Canada.

Poon, C., Laliberté, J. and Straznicky, P.V. "Review of Fibre-Metal-Laminate Technology," $3^{\text {rd }}$ Joint Canada-Japan Workshop on Composites, 2000, Kyoto, Japan.

Poston, K. "Impact Properties and Related Applications of Fibre-metal-laminates," Structural Laminates Corporation memorandum TD-M-94-007, 1994, also presented at $15^{\text {th }}$ European SAMPE Conference.

Russell, A.J. and Street, K.N., "On the Development of a Mixed Mode Tensile-Shear Interlaminar Fracture Criterion for Graphite-Epoxy Composites," $10^{\text {th }}$ Canadian Fracture Conference, 1983, Waterloo, Canada.

Shi, G. and Poon, C. "Investigations of buckling and post-buckling behaviour of stiffened FML panels", International Conference on Computational Engineering \& Science (ICES'01), 2001, Mexico.

Sun, C.T., Dicken, A. and Wu, H.F. "Characterization of Impact Damage in ARALL Laminates." Composites Science and Technology, 1993, v. 49, n. 2, pp. 139-144.

Vlot, A. "Impact Tests on Aluminum 2024-T3, Aramid and Glass Reinforced Laminates and Thermoplastic Composites." TUDelft Report Number LR-534, 1987.

Vlot, A. "Low-Velocity Impact Loading on Fibre Reinforced Aluminum Laminates (ARALL and GLARE) and other aircraft sheet materials." TUDelft Report Number LR718, March 1993a.

Vlot, A. "Impact Properties of Fibre-metal-laminates." Composites Engineering, 1993b, v. 3, n. 10, pp. 911-924. 
Vlot, A. "Impact Loading on Fibre-metal-laminates." Journal of Impact Engineering, 1996, v. 18, n. 3, pp. 291-308.

Vlot, A., Kroone, E. and Larocca. "Impact Response of Fibre-metal-laminates," Key Material Technologies, 1998, v. 141-143, pp. 235-276.

Vlot, A and Ingen, J. W. van, "Delamination Resistance of Post-Stretched Fibre Metal Laminates," Journal of Composite Materials, 1998, v. 32, n. 19, pp. 1784-1805.

Vogelsang, L.B., Marissen, R. and Schijve, J. "A New Fatigue Resistant Material: Aramid Reinforced Aluminum Laminate (ARALL)," $11^{\text {th }}$ ICAF Symposium, 1981, Noordwijkerhout, the Netherlands.

Vries, T.J. de, Vlot, A. and Hashagen, F. "Delamination Behavior of Spliced Fiber Metal Laminates Part 1. Experimental Results," Composite Structures, 1999, v. 46, pp. 131-145.

Whitney, J., "Experimental Characterization of Delamination Fracture" Interlaminar Response of Composite Materials, Composite Materials Series by Pagano, N.J., 1989, pp. 161-250.

Williams, K. and Vaziri, R. "Finite Element Analysis of the Impact Response of CFRP Composite Plates," ICCM-10, 1995, Whistler, Canada, v. 5, pp. 647-654.

Williams, K. and Vaziri, R. "Application of a Damage Mechanics Model for Predicting the Impact Response of Composite Materials," Computers and Structures, 2001, v. 79, pp. 997-1011.

Wu, H.F. and Sun, C.T. "Impact Damage Characterization of Aramid Aluminum Laminates." ICCM-9, 1993, Madrid, Spain.

Yeh, J.R. "Fracture Mechanics of Delaminations in ARALL Laminates," Engineering Fracture Mechanics, 1988, v. 30, n. 6, pp. 827-837. 
APPENDICES 


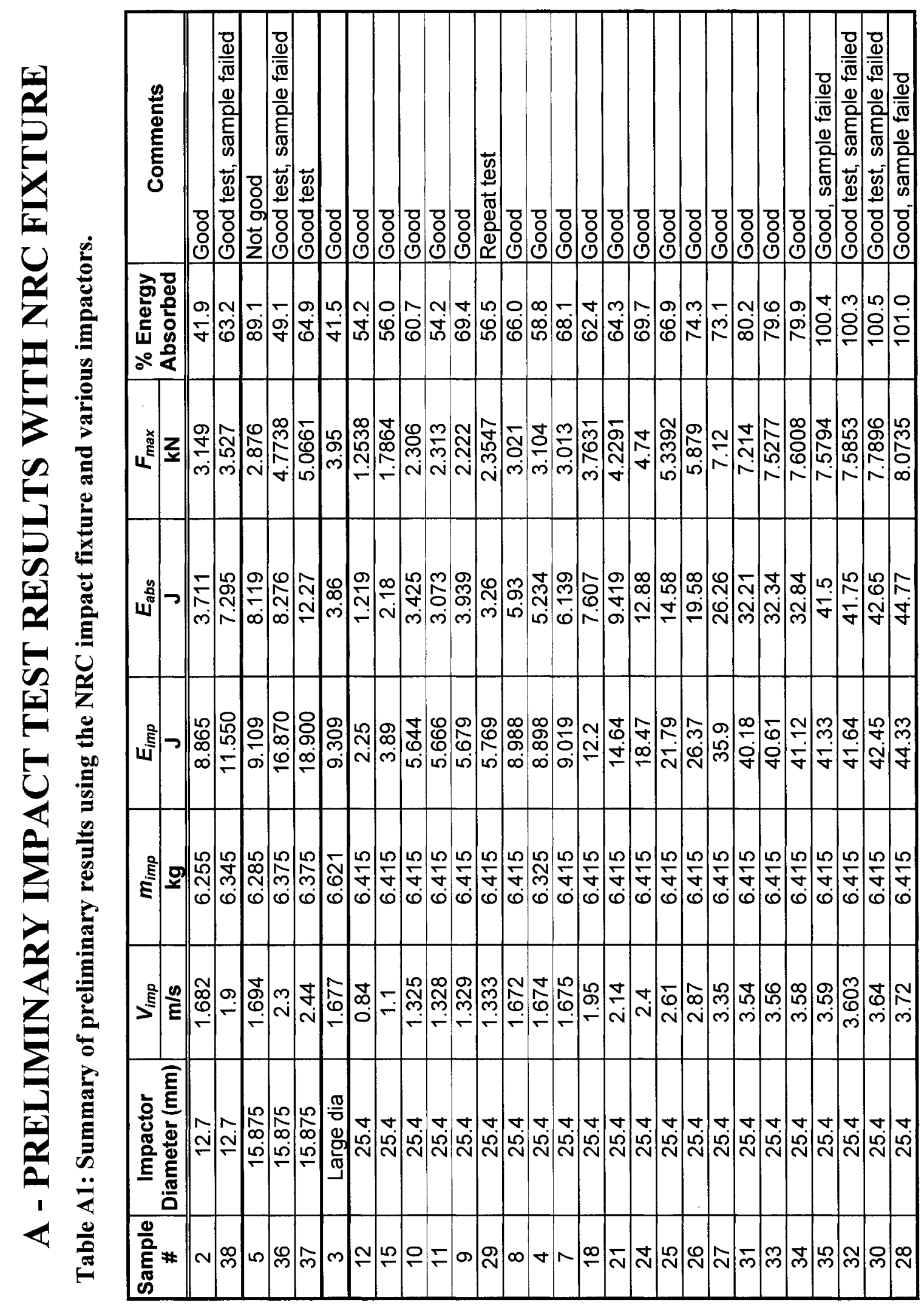




\section{B - PRELIMINARY IMPACT TEST RESULTS WITH NASA AND NRC TEST FIXTURES}

Table B1: Preliminary impact test results using the NRC and NASA fixtures with $25.4 \mathrm{~mm}$ and $12.7 \mathrm{~mm}$ diameter impactors, respectively.

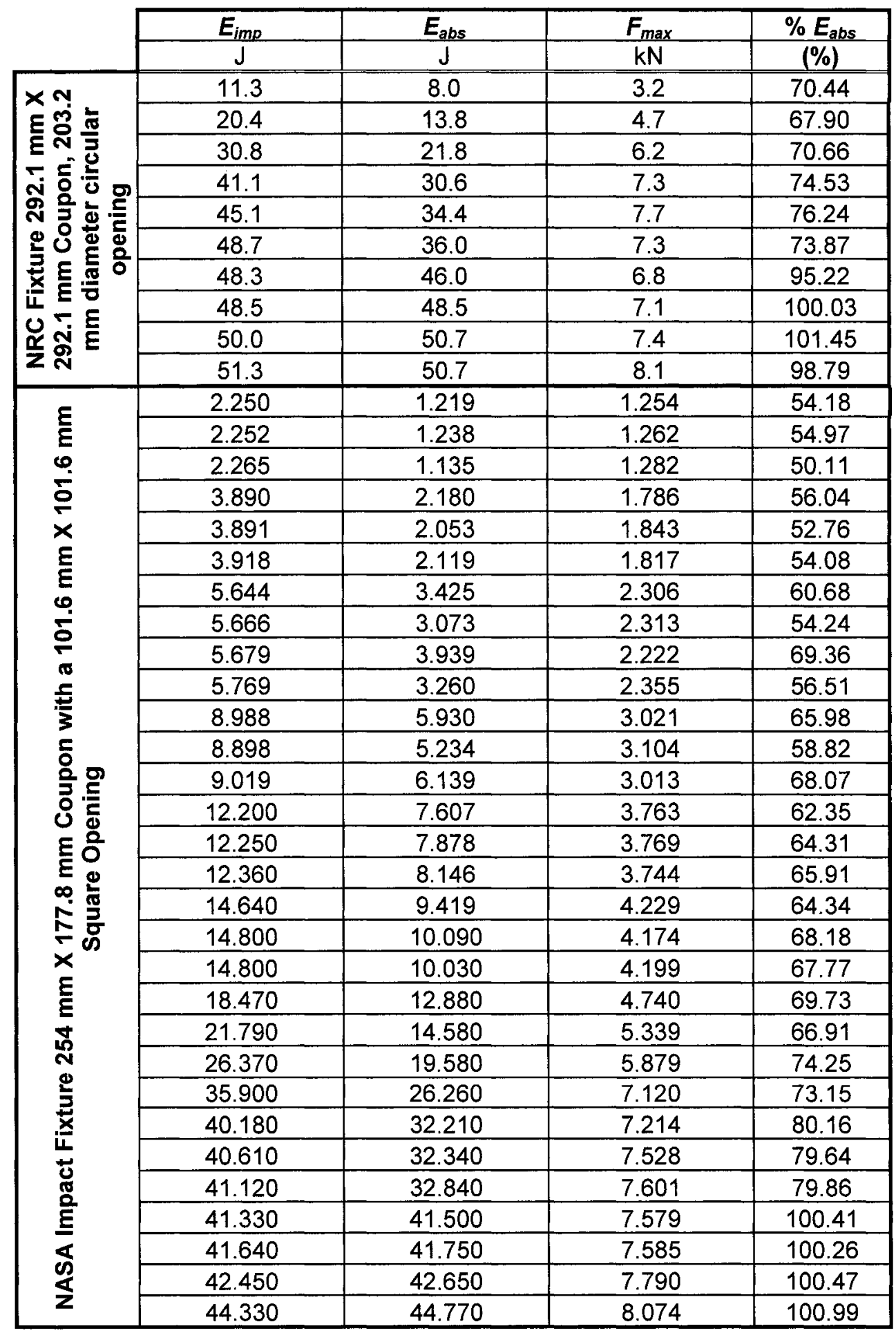




\section{C - NRC AND NASA IMPACT FIXTURE RESULTS}

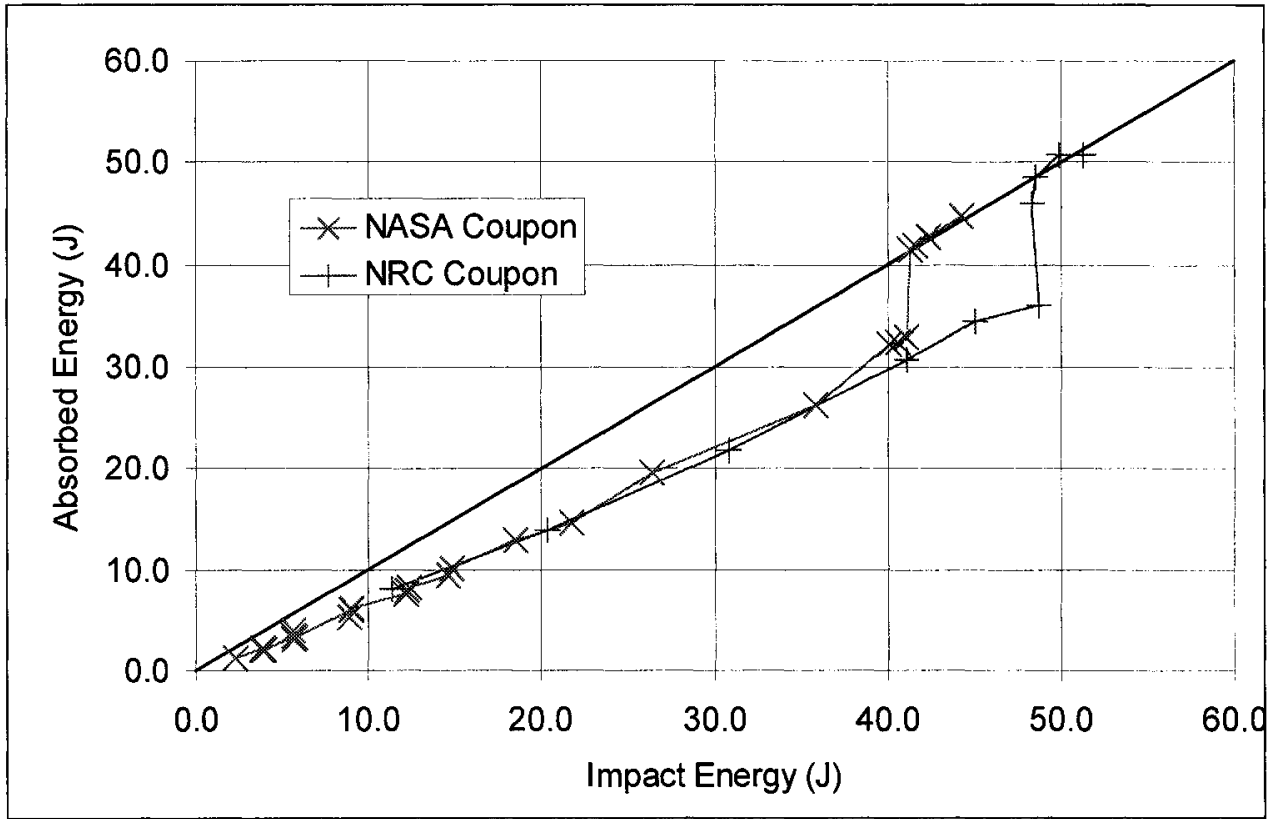

Figure C1: Absorbed energy for the NASA and NRC impact fixtures.

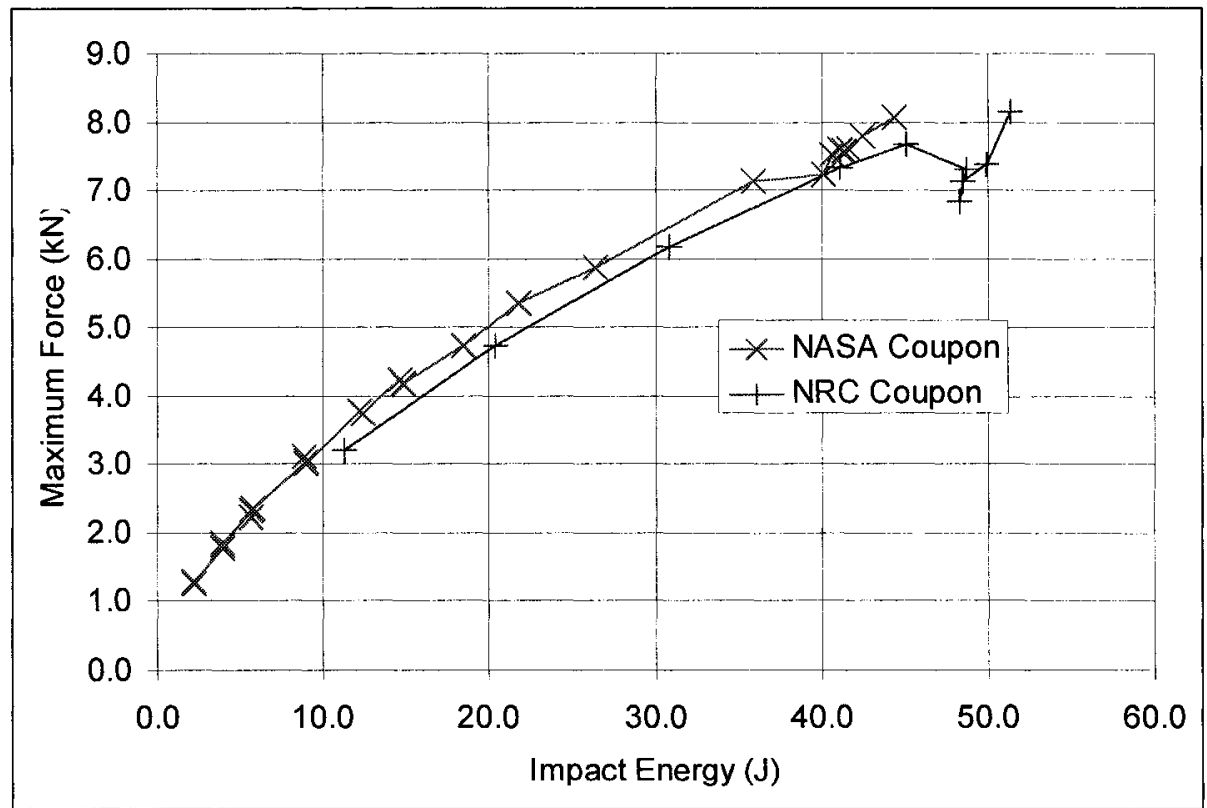

Figure C2: Maximum force versus impact energy for the NASA and NRC fixtures. 


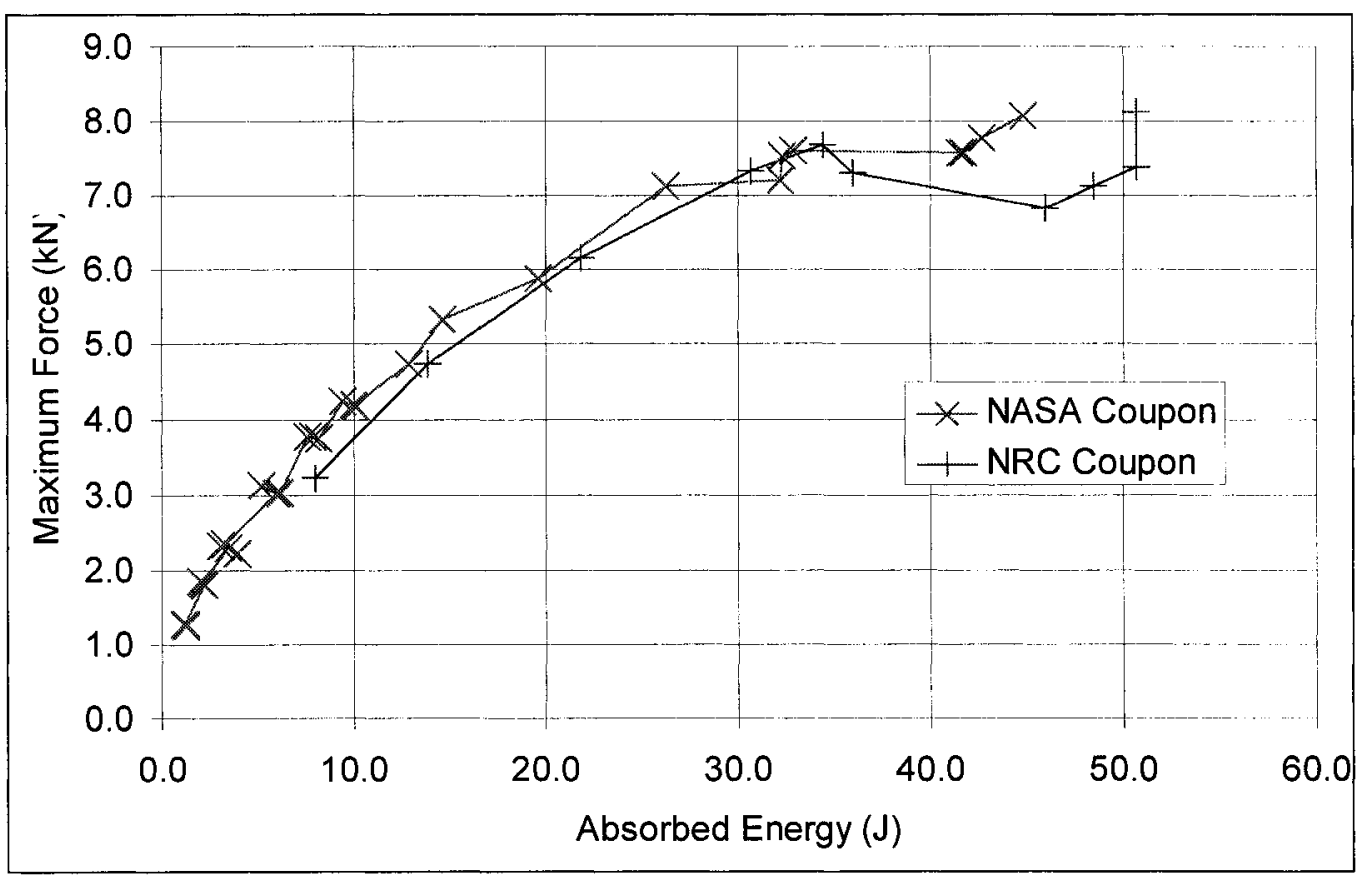

Figure C3: Maximum force versus absorbed energy for the NASA and NRC impact fixtures. 


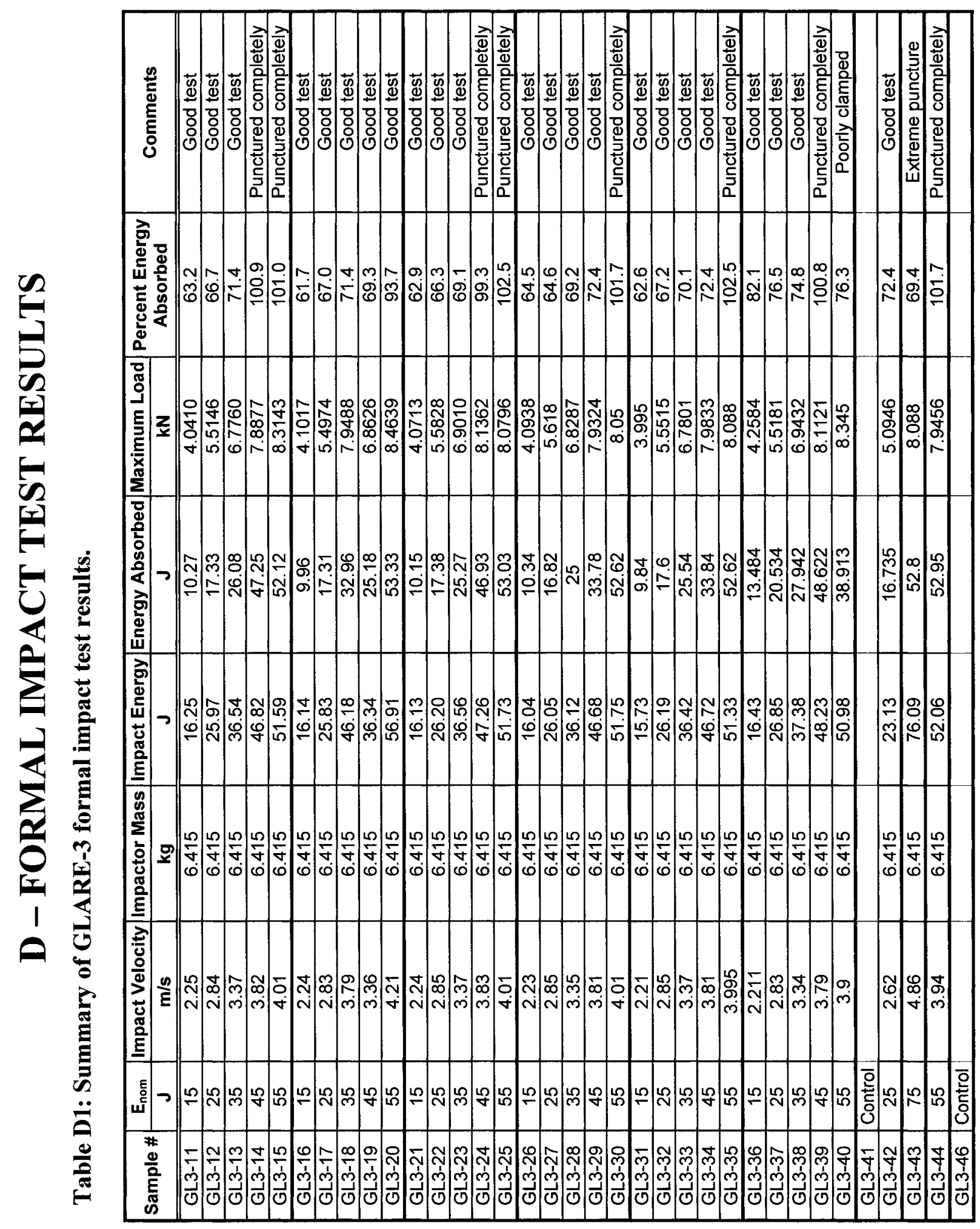




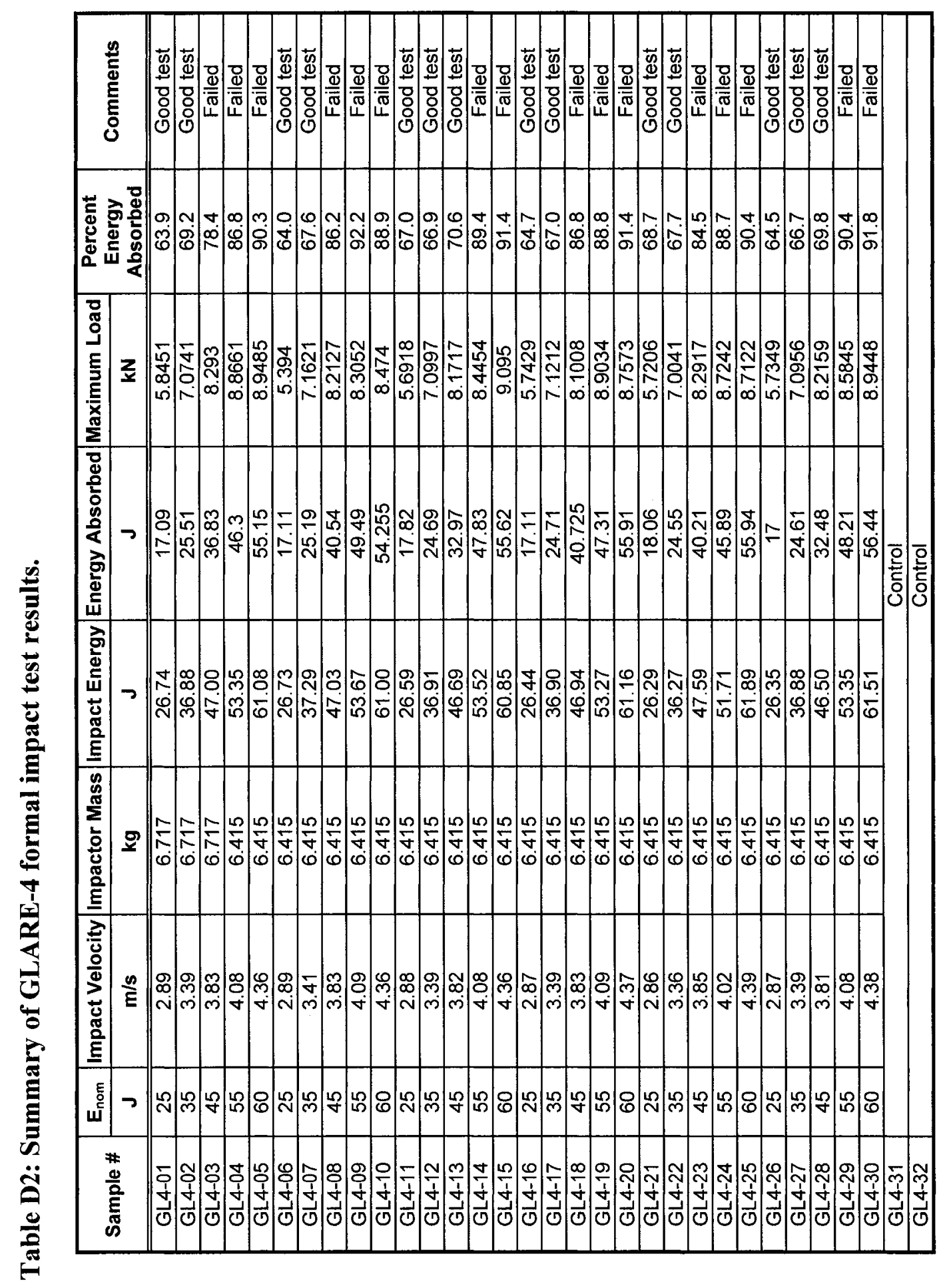




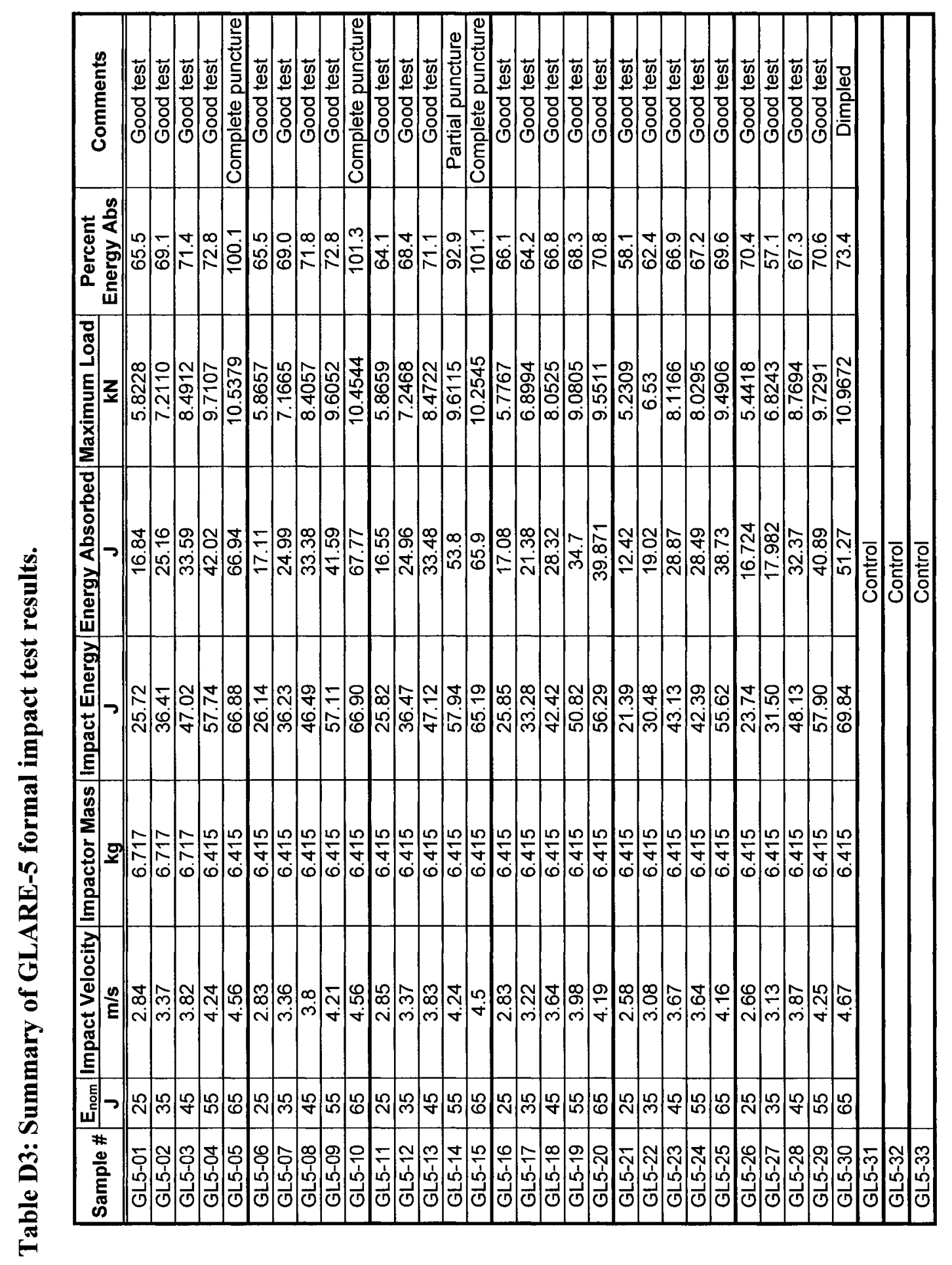




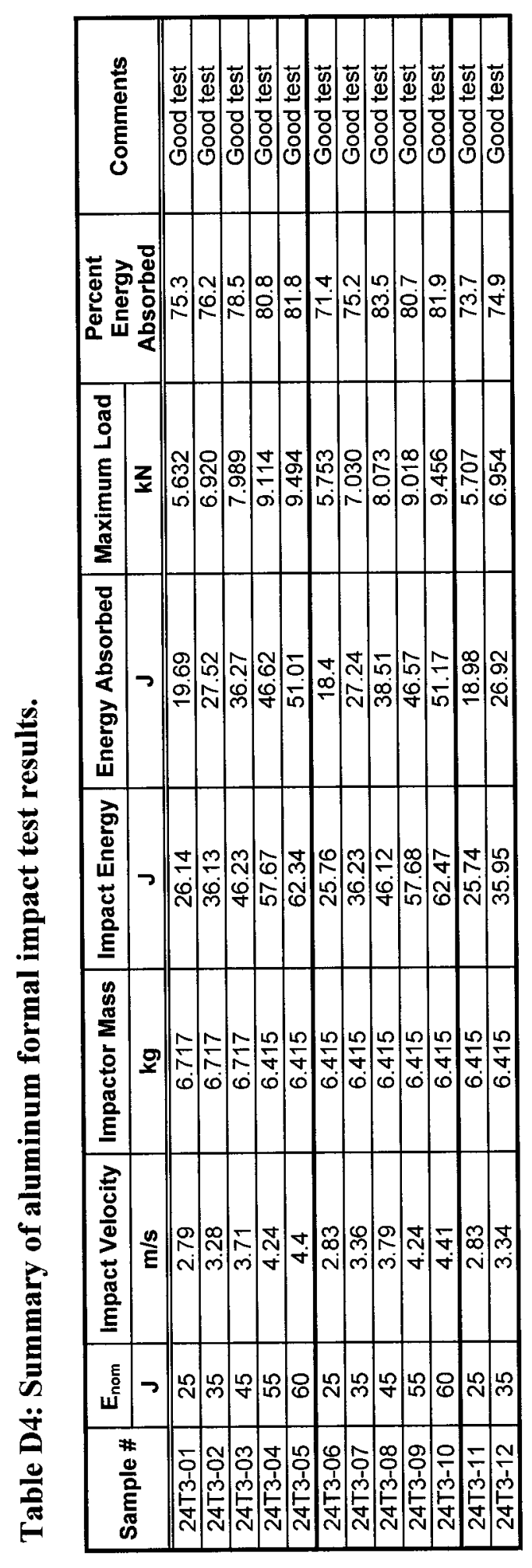

$\frac{\text { N }}{4}$ 


\section{E - IMPACT DAMAGE PHOTOS}

Damage due to drilling
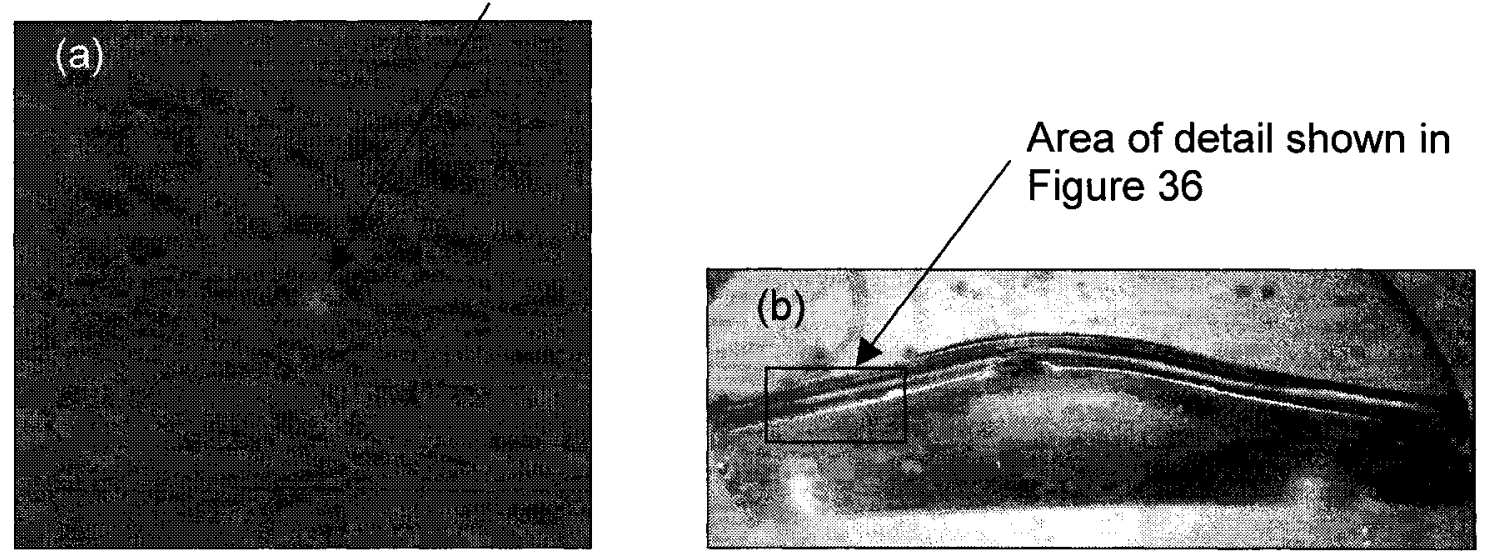

Figure E1: Sample GLARE-3-31 (15.73 J) (a) x-ray and (b) cross-section.

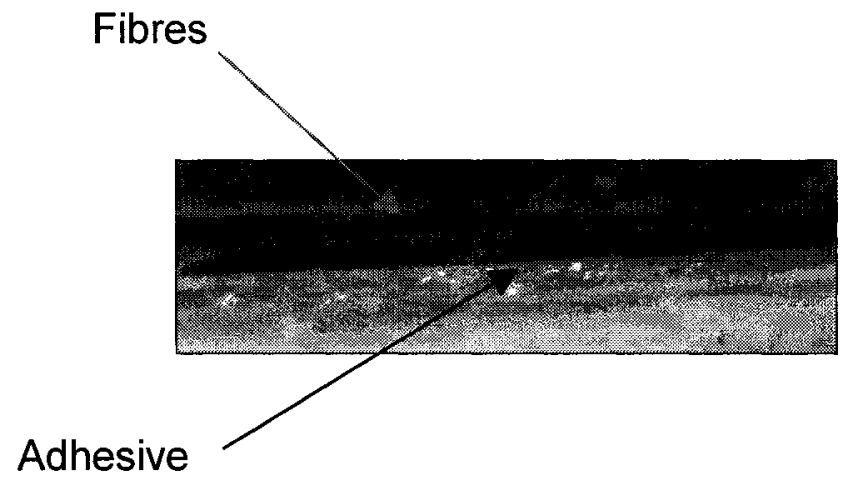

Figure E2: GLARE-3-31 (15.73 J) fibre delamination.
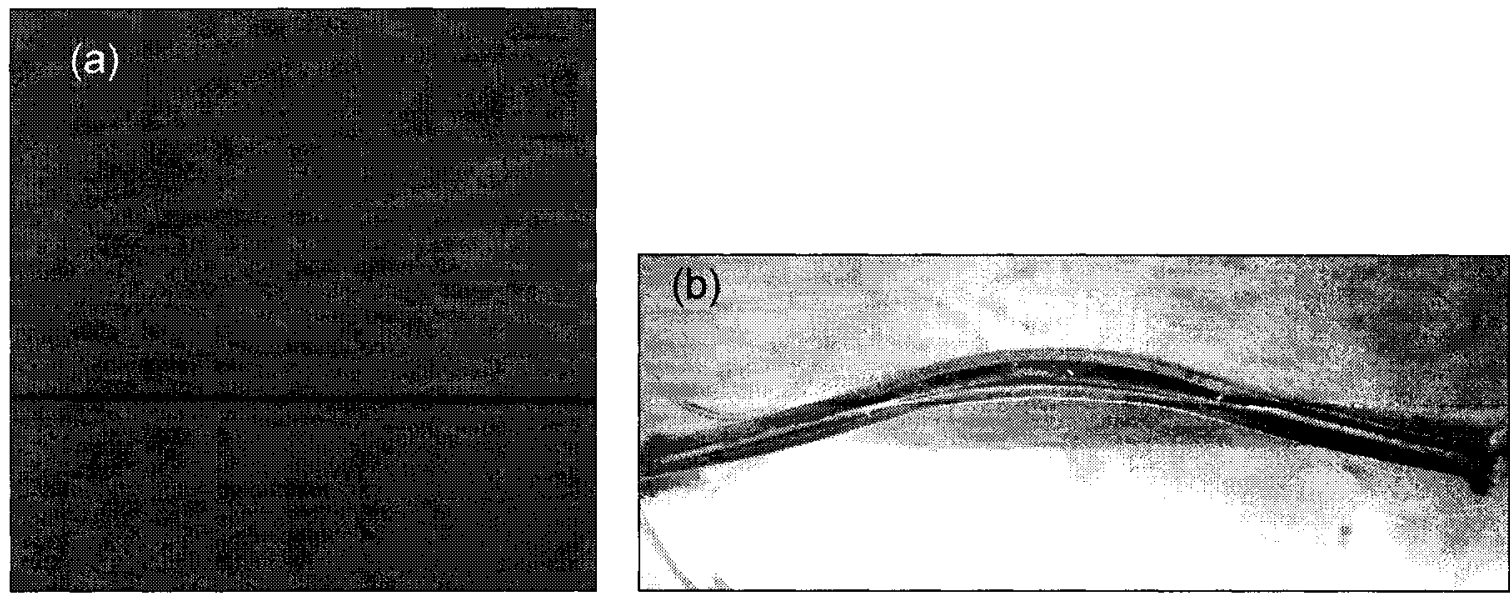

Figure E3: GLARE-3-32 (26.19 J) (a) x-ray and (b) cross-section. 

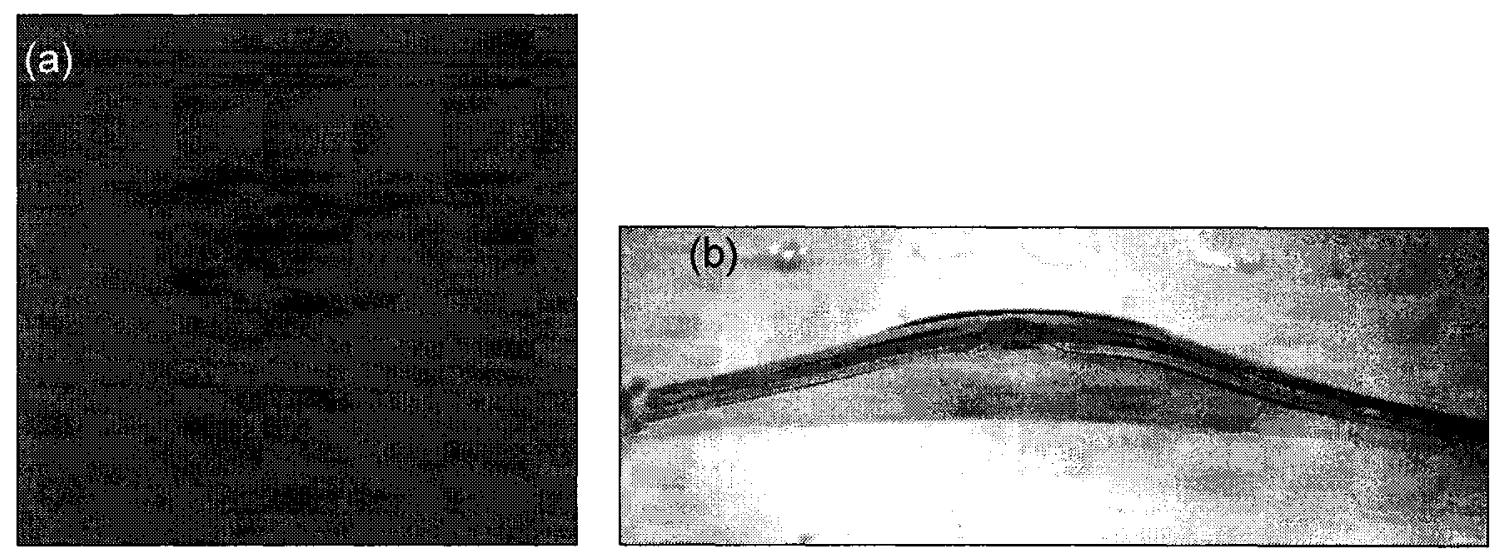

Figure E4: GLARE-3-33 (36.42 J) (a) x-ray and (b) cross-section.

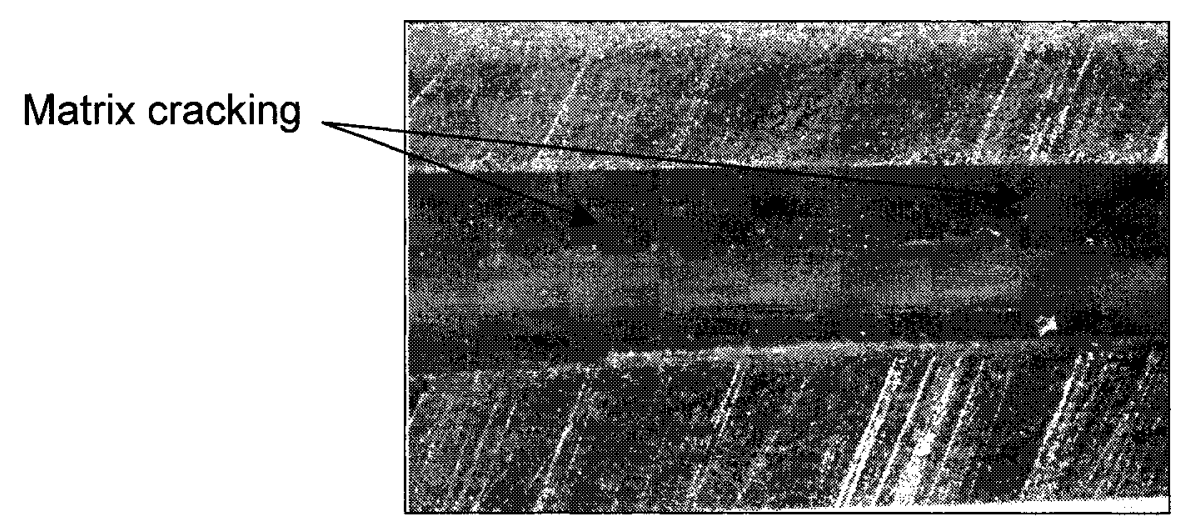

Figure E5: Matrix cracking in 3-33 in the $90^{\circ}$ layer.

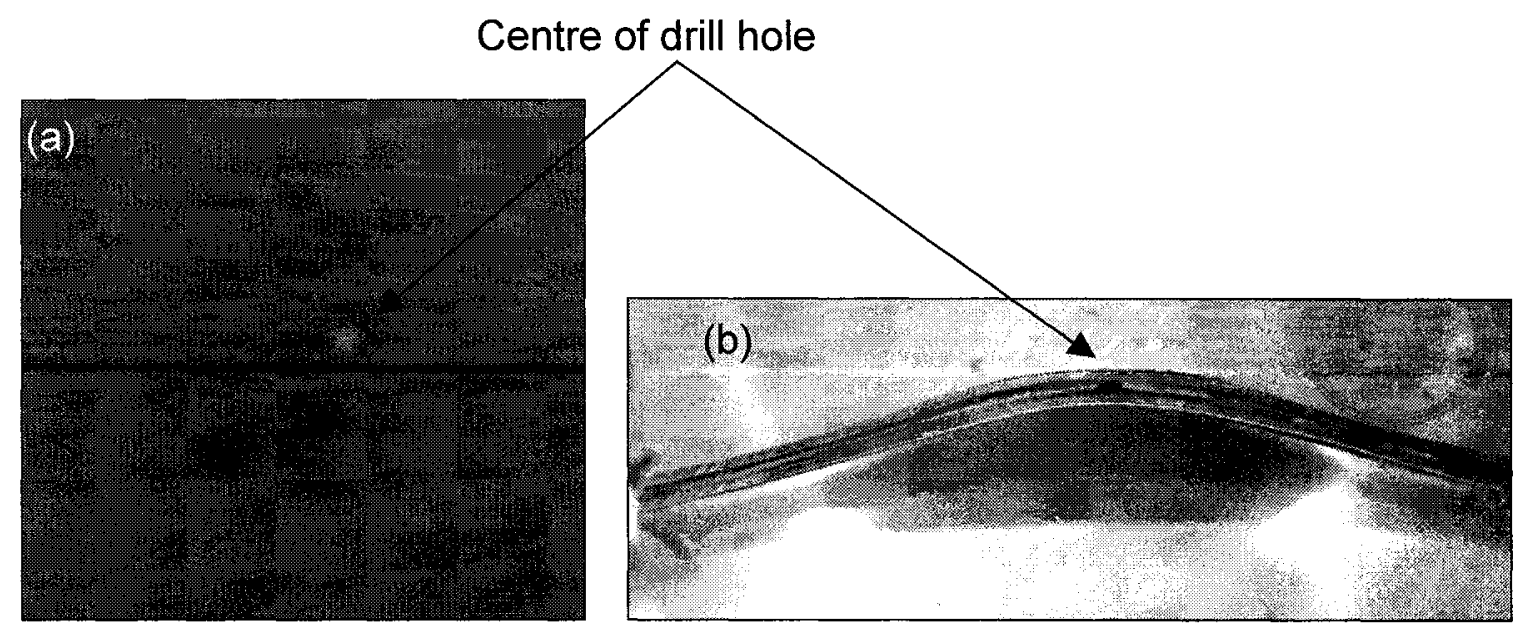

Figure E6: GLARE-3-34 (46.72 J) (a) x-ray and (b) cross-section. 
Matrix cracking

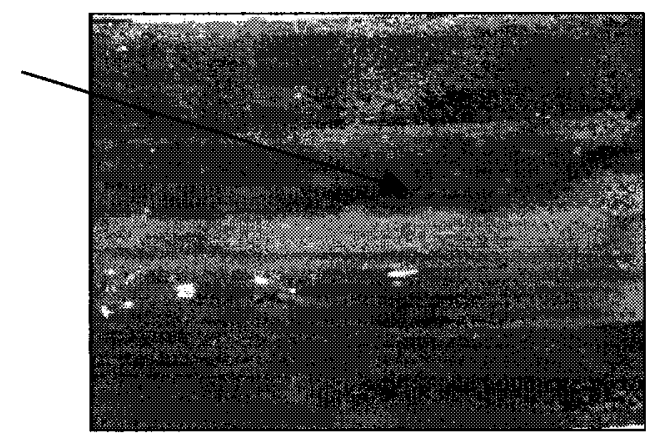

Figure E7: Intralaminar cracking in GLARE-3-34 (46.72 J).

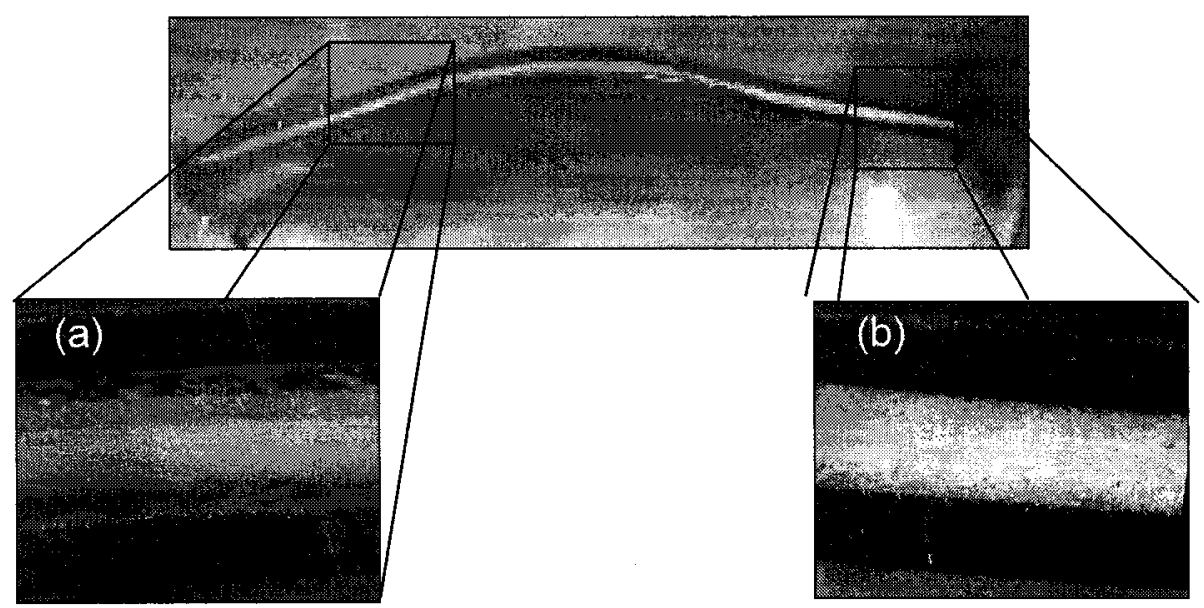

Figure E8: (a) Delamination in GLARE-4-16 and (b) unaffected region.

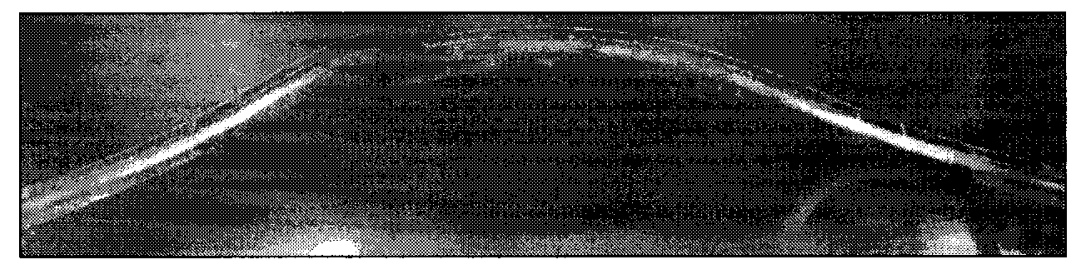

Figure E9: GLARE-4-08 (47.03 J) cross-section. 


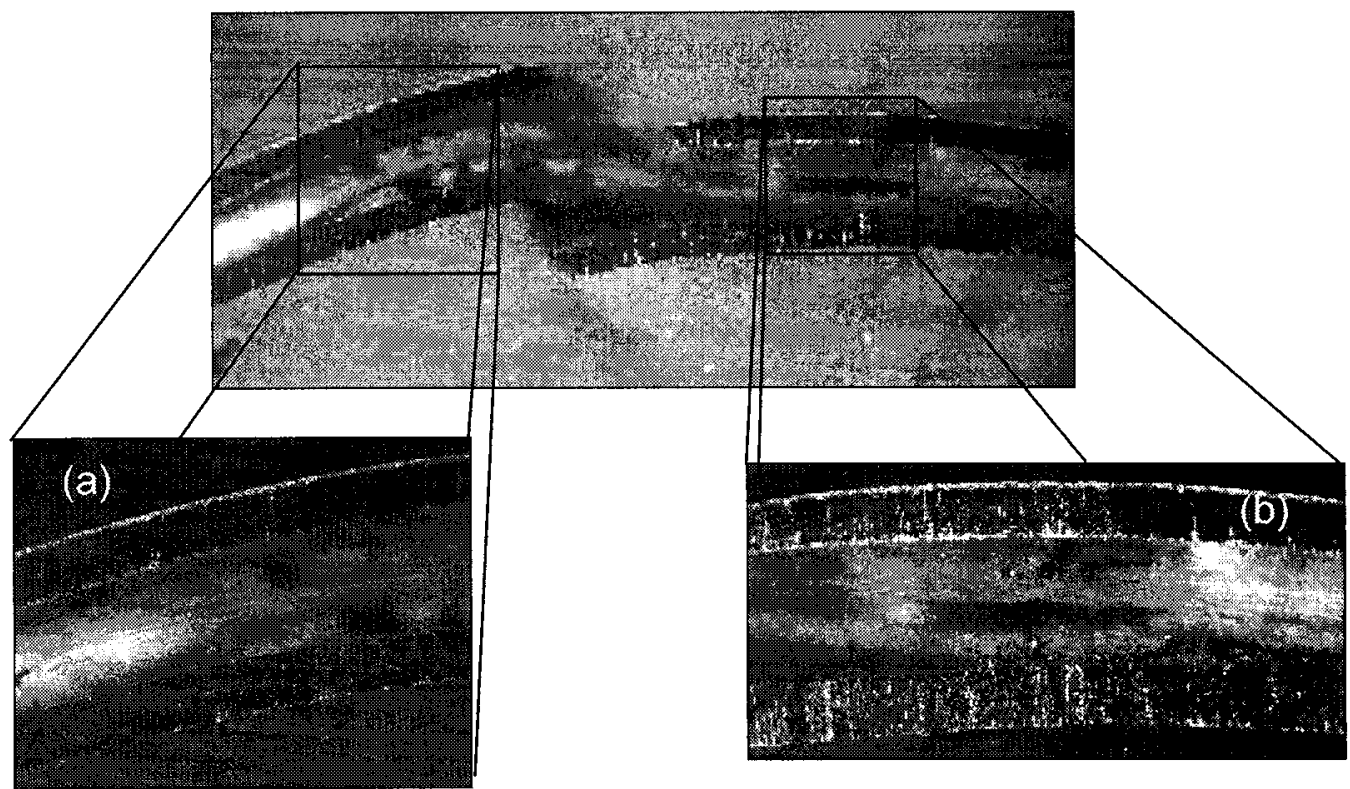

Figure E10: Through-crack in GLARE-4-08 (47.03 J).

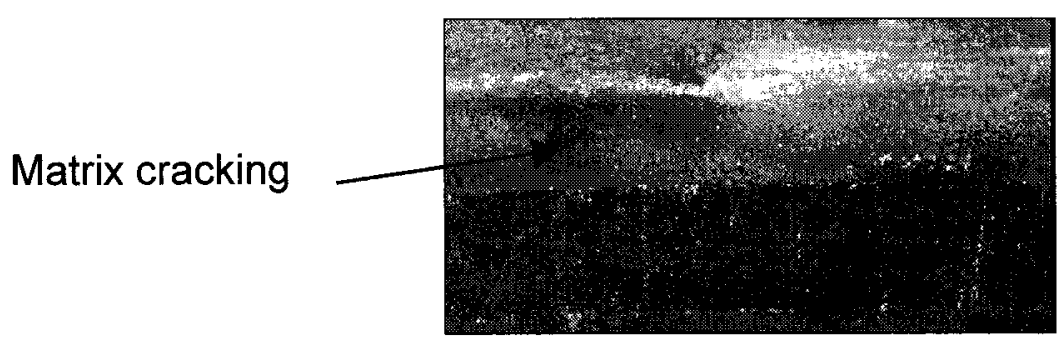

Figure E11: Matrix cracking in GLARE-4-08 (47.03 J)

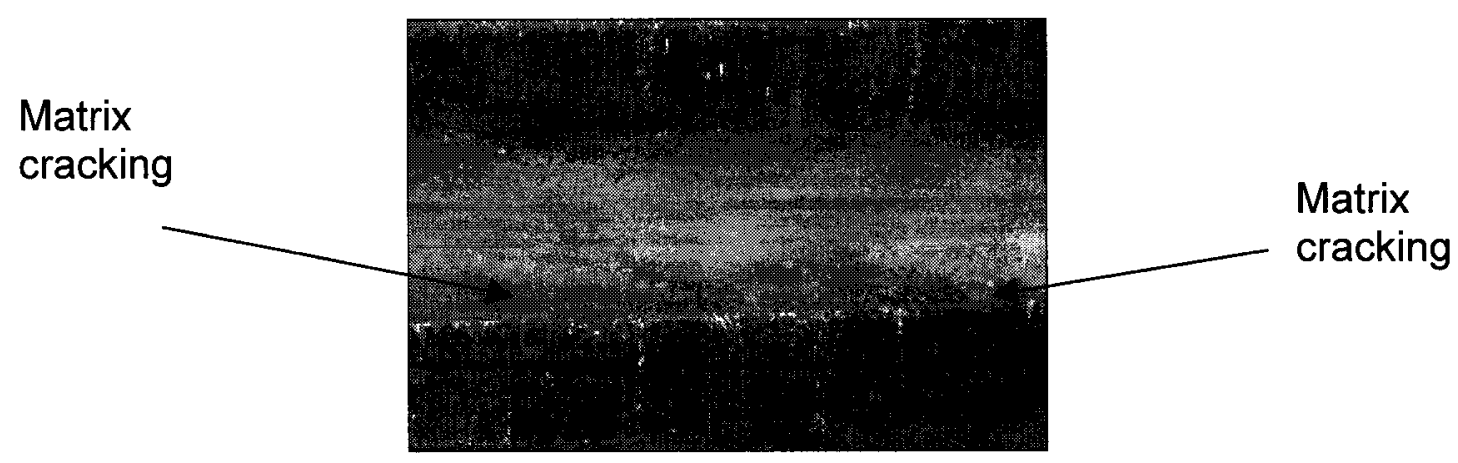

Figure E12: Matrix cracking in GLARE-4-27 (36.88 J). 


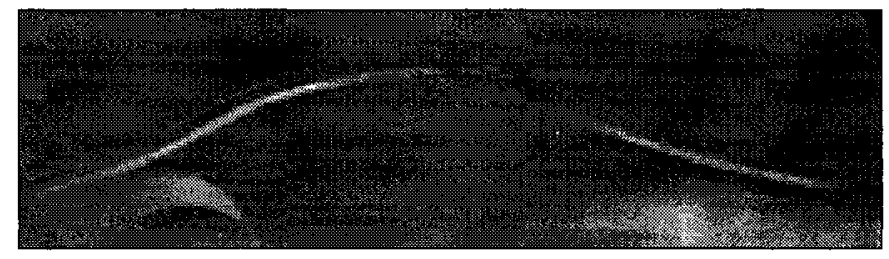

Figure E13: GLARE-4-19 (53.27 J) cross-section.

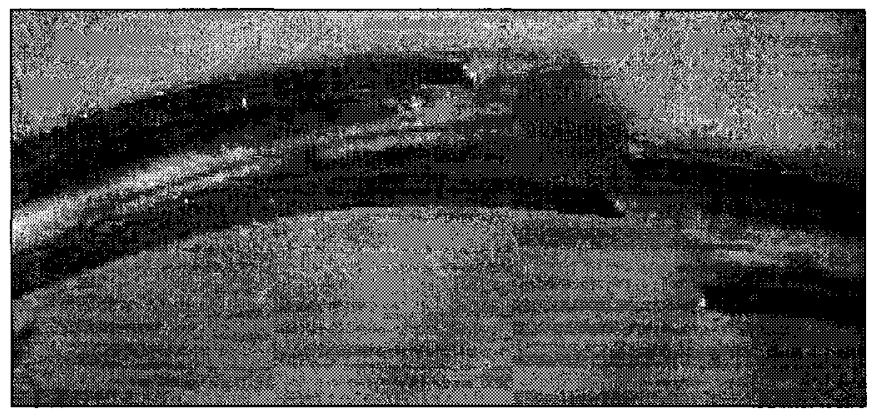

Figure E14: GLARE-4-19 (53.27 J) detail of cracked area.

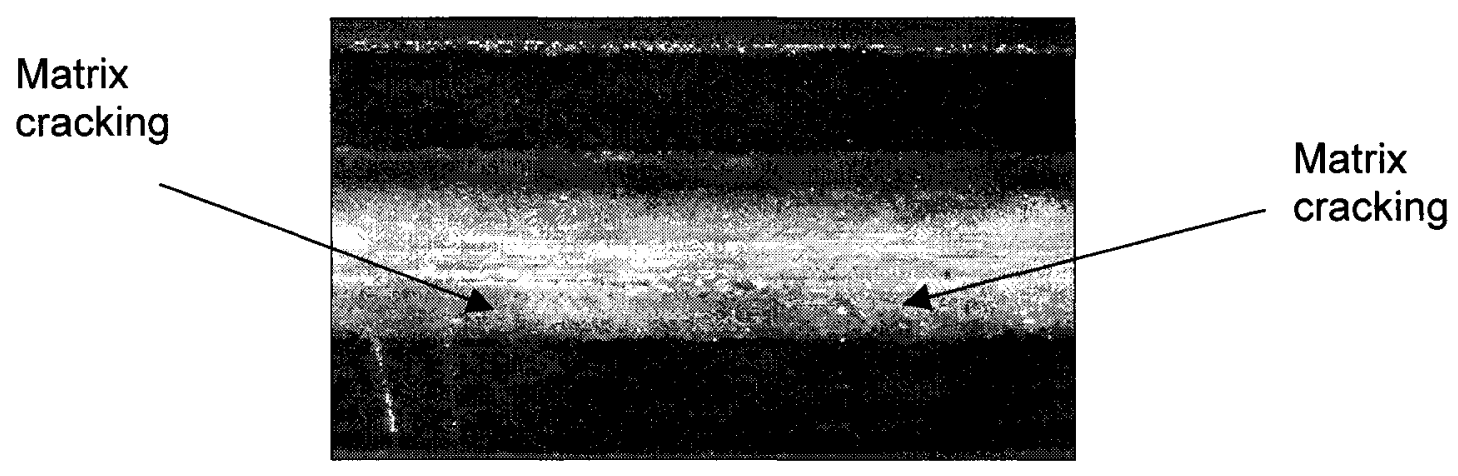

Figure E15: Matrix cracking and intralaminar failures in GLARE-4-19. 


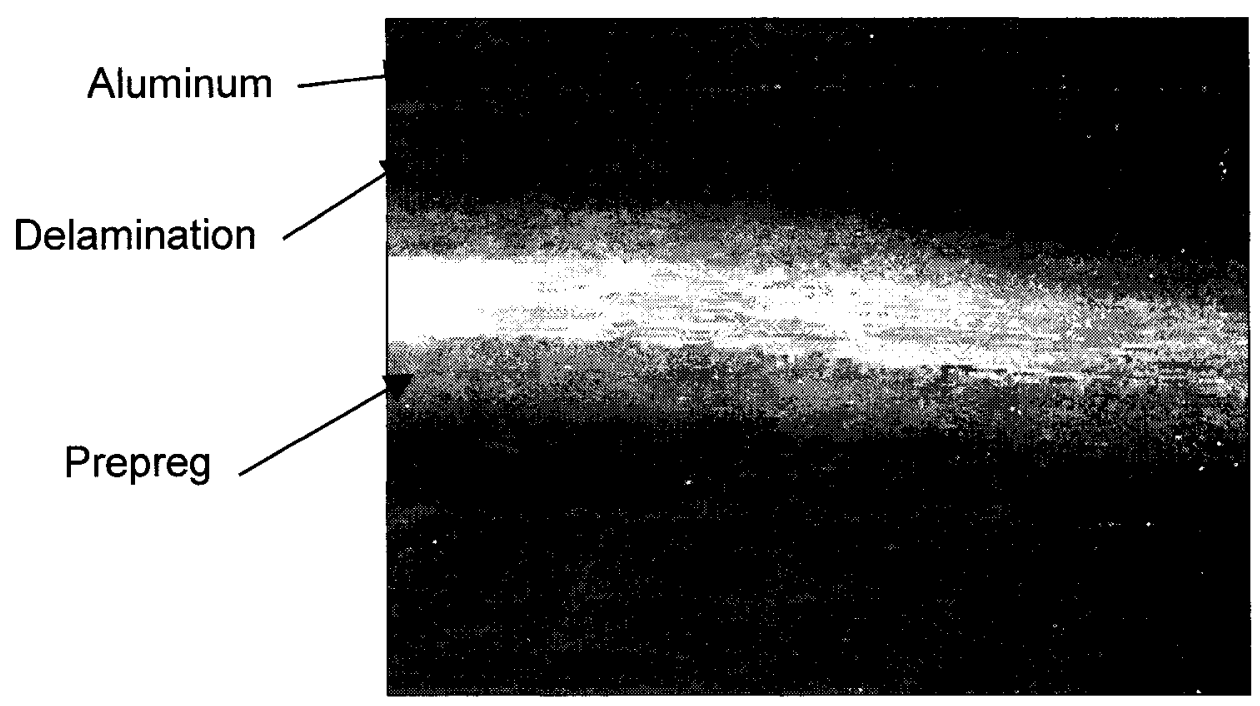

Figure E16: Delaminated regions in prepreg layers of GLARE-4-19.
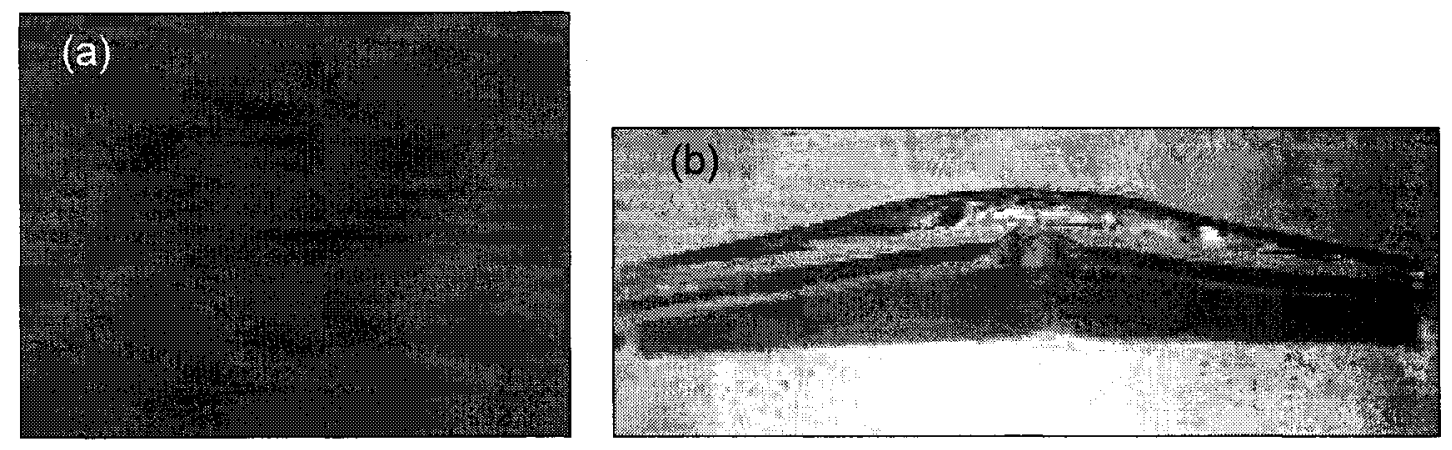

Figure E17: GLARE-5-04 (55 J) (a) x-ray and (b) cross-section.
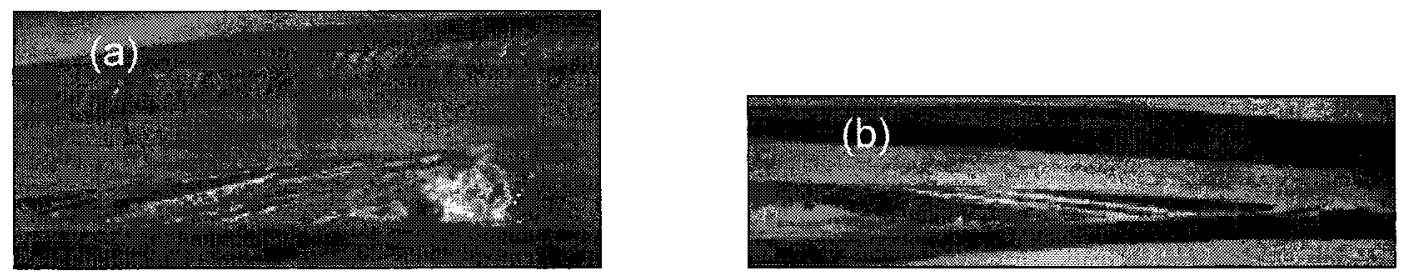

Figure E18: Delamination and fibre-bridging in GLARE-5-04 on (a) left and (b) right of dent. 


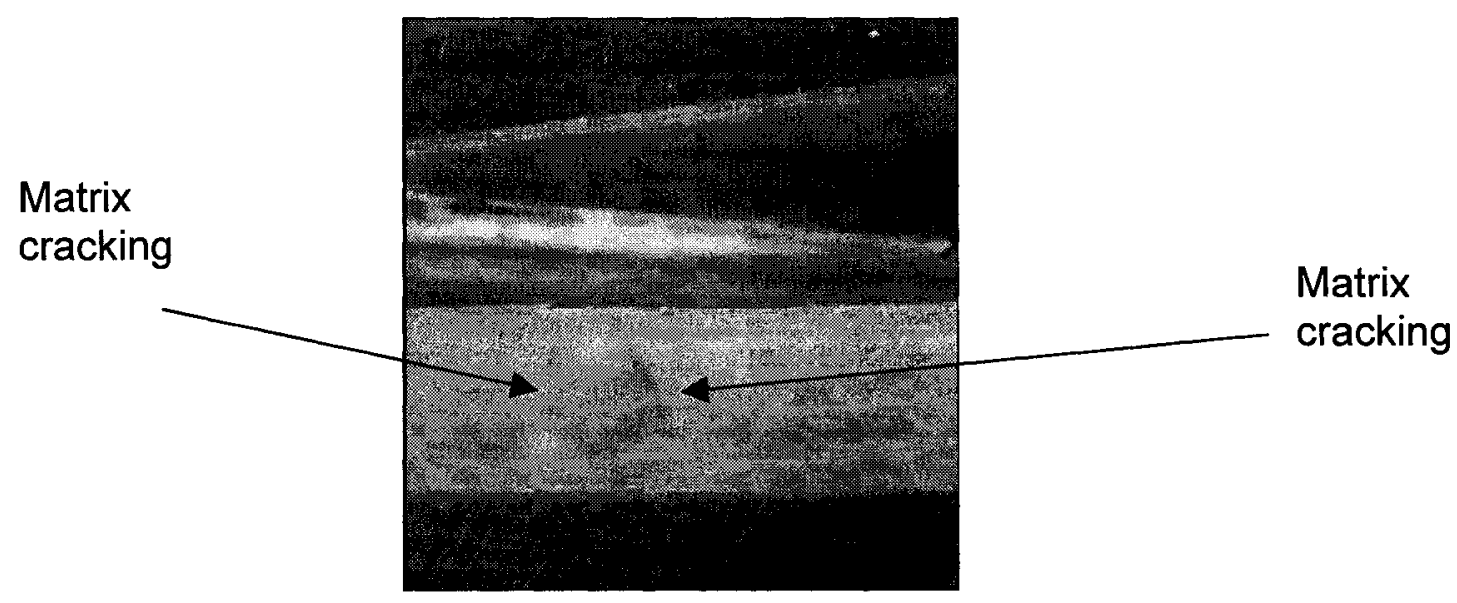

Figure E19: Matrix cracking in GLARE-5-04.
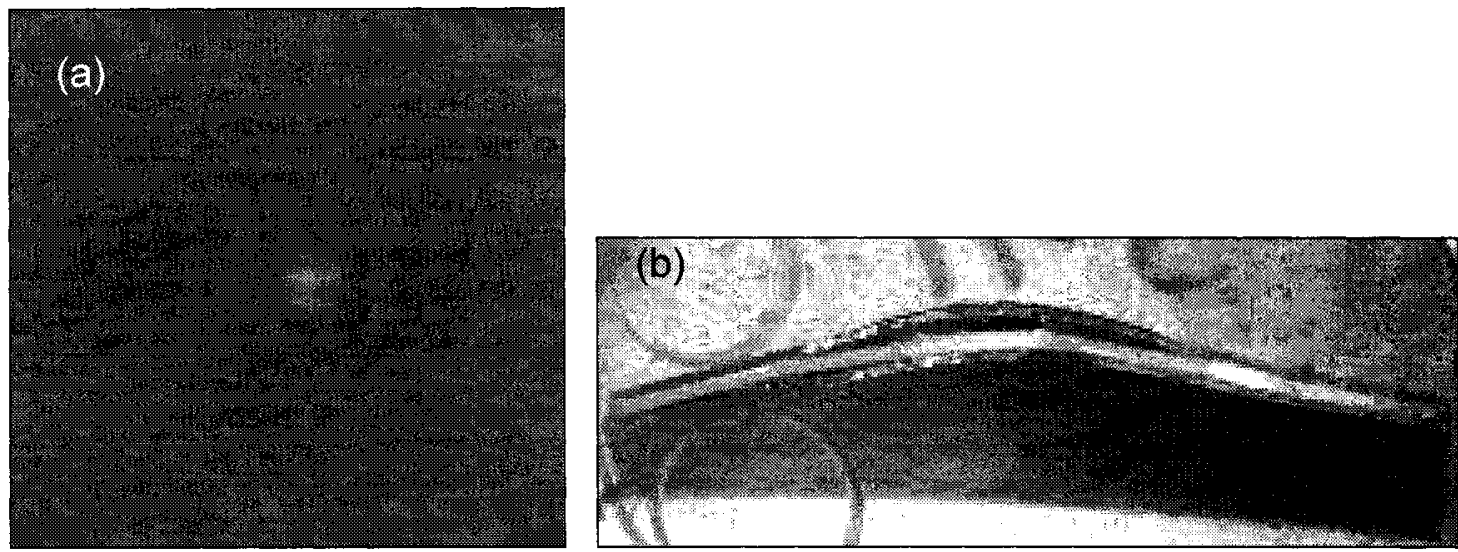

Figure E20: GLARE-5-11 (25 J) (a) x-ray and (b) cross-section.

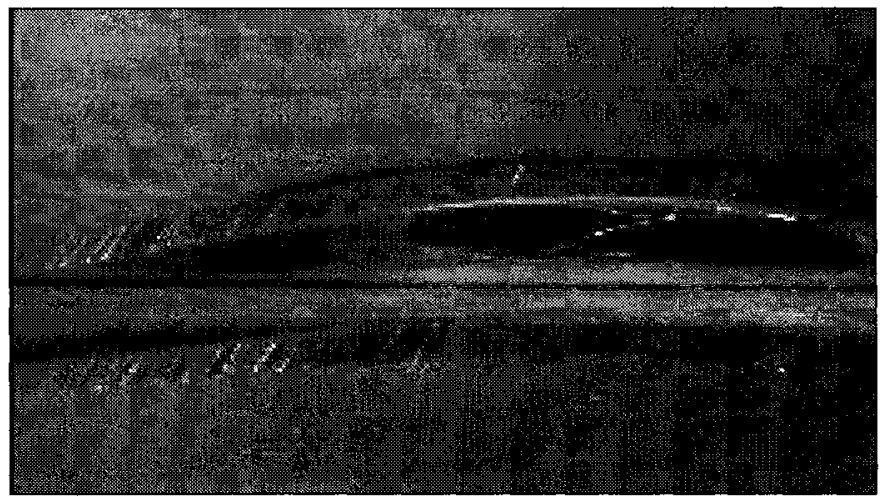

Figure E21: GLARE-5-11 showing delamination damage. 

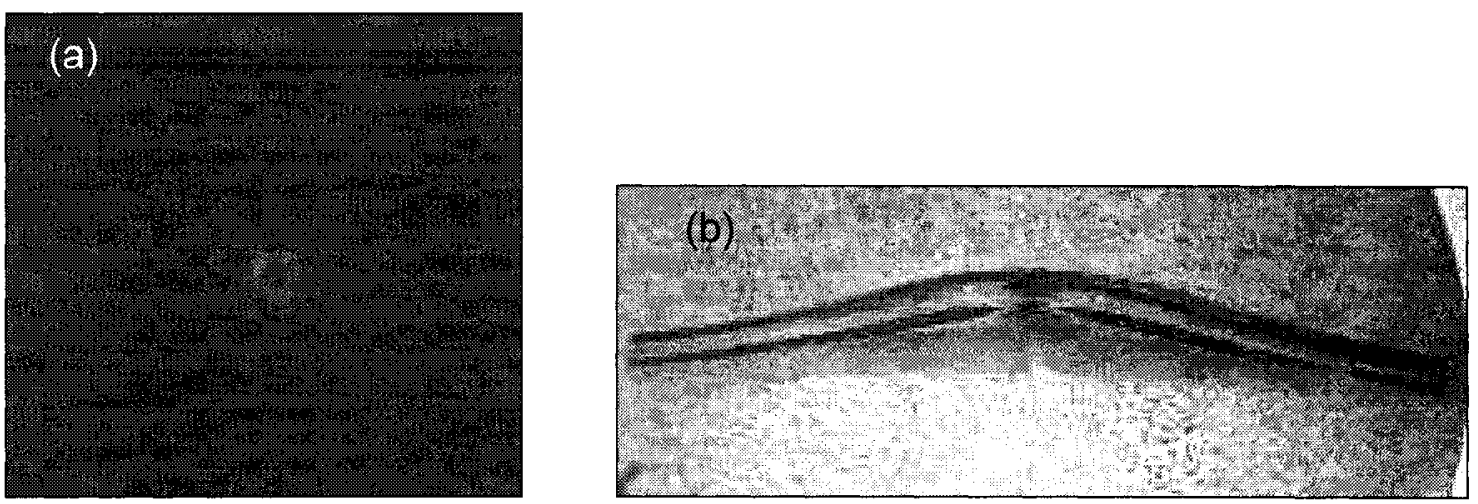

Figure E22: GLARE-5-12 (35 J) (a) x-ray and (b) cross-section.

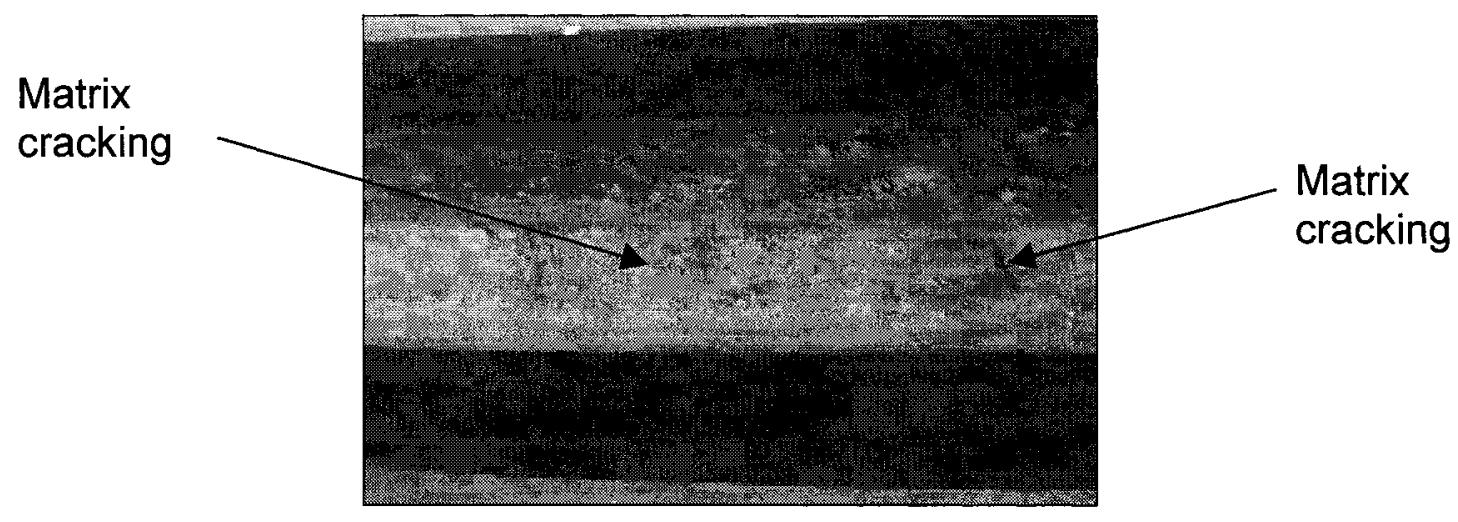

Figure E23: GLARE-5-12 matrix cracking and top $90^{\circ}$ layer adhesive failure. 


\section{F - ALUMINUM IMPACT SIMULATION RESULTS}

Table F1: Summary of peak force results for preliminary aluminum simulations.

\begin{tabular}{|c|c|c|c|c|c|c|c|}
\hline & Velocity & Mass & $E_{i m p}$ & $\left(F_{\text {max }}\right)_{\exp }$ & $\left(F_{\max }\right)_{\operatorname{sim}}$ & \multirow{2}{*}{$\begin{array}{c}\text { Percent } \\
\text { Difference }\end{array}$} \\
\hline & & $\mathrm{m} / \mathrm{s}$ & $\mathbf{k g}$ & $\mathrm{J}$ & $\mathbf{k N}$ & $\mathbf{k N}$ & \\
\hline \multirow{5}{*}{\multicolumn{2}{|c|}{ 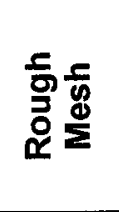 }} & 2.88 & 6.45 & 26.8 & $\overline{5.70}$ & 6.25 & 9.71 \\
\hline & & 3.38 & 6.45 & 36.8 & 6.97 & 7.4 & 6.20 \\
\hline & & 3.81 & 6.45 & 46.7 & 8.03 & 8.65 & 7.71 \\
\hline & & 4.23 & 6.45 & 57.8 & 9.07 & 10.2 & 12.51 \\
\hline & & 4.40 & 6.45 & 62.4 & 9.47 & 10.4 & 9.76 \\
\hline \multirow{5}{*}{\multicolumn{2}{|c|}{ 超蛋 }} & 2.88 & 6.45 & 26.8 & 5.70 & 6 & 5.32 \\
\hline & & 3.38 & 6.45 & 36.8 & 6.97 & 7.5 & 7.64 \\
\hline & & 3.81 & 6.45 & 46.7 & 8.03 & 8.8 & 9.57 \\
\hline & & 4.23 & 6.45 & 57.8 & 9.07 & 10 & 10.30 \\
\hline & & 4.40 & 6.45 & 62.4 & 9.47 & 10.4 & 9.76 \\
\hline \multirow{10}{*}{ 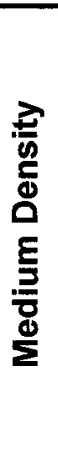 } & \multirow{5}{*}{$\frac{\frac{0}{a}}{\frac{\xi}{\omega}}$} & 2.88 & 6.45 & 26.8 & 5.70 & 6.2 & 8.83 \\
\hline & & 3.38 & 6.45 & 36.8 & 6.97 & 8 & 14.81 \\
\hline & & 3.81 & 6.45 & 46.7 & 8.03 & 9.1 & 13.31 \\
\hline & & 4.23 & 6.45 & 57.8 & 9.07 & 10.2 & 12.51 \\
\hline & & 4.40 & 6.45 & 62.4 & 9.47 & 10.6 & 11.87 \\
\hline & \multirow{5}{*}{$\begin{array}{l}\frac{8}{\mathbb{d}} \\
\frac{2}{2} \\
\frac{\pi}{0} \\
0\end{array}$} & 2.88 & 6.45 & 26.8 & 5.70 & 6.6 & 15.85 \\
\hline & & 3.38 & 6.45 & 36.8 & 6.97 & 7.9 & 13.38 \\
\hline & & 3.81 & 6.45 & 46.7 & 8.03 & 8.6 & 7.08 \\
\hline & & 4.23 & 6.45 & 57.8 & 9.07 & 10.1 & 11.41 \\
\hline & & 4.40 & 6.45 & 62.4 & 9.47 & 10.4 & 9.76 \\
\hline \multirow{5}{*}{\multicolumn{2}{|c|}{ 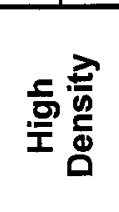 }} & 2.88 & 6.45 & 26.8 & 5.70 & 6.25 & 9.71 \\
\hline & & 3.38 & 6.45 & 36.8 & 6.97 & 7.4 & 6.20 \\
\hline & & 3.81 & 6.45 & 46.7 & 8.03 & 8 & -0.39 \\
\hline & & 4.23 & 6.45 & 57.8 & 9.07 & 9.6 & 5.89 \\
\hline & & 4.40 & 6.45 & 62.4 & 9.47 & 9.75 & 2.90 \\
\hline
\end{tabular}


Table F2: Dent depth results for simulated impact events for preliminary aluminum impact simulations.

\begin{tabular}{|c|c|c|c|c|c|c|}
\hline & \multicolumn{3}{|c|}{ Event Parameters } & \multicolumn{3}{|c|}{ Dent Depth Results } \\
\hline & \multirow{2}{*}{ Velocity } & \multirow{2}{*}{ Mass } & \multirow{2}{*}{$\begin{array}{l}\text { Impact } \\
\text { Energy }\end{array}$} & Experimental & Simulation & \multirow{3}{*}{$\begin{array}{c}\text { Percent } \\
\text { Difference }\end{array}$} \\
\hline & & & & Permanent & Permanent & \\
\hline & $\mathrm{m} / \mathrm{s}$ & $\mathbf{k g}$ & $\mathrm{J}$ & $\mathbf{m m}$ & $\mathbf{m m}$ & \\
\hline \multirow{5}{*}{ 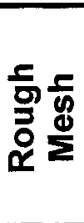 } & 2.88 & 6.45 & 26.8 & 6.50 & 7.00 & 7.75 \\
\hline & 3.38 & 6.45 & 36.8 & 7.67 & 8.30 & 8.17 \\
\hline & 3.81 & 6.45 & 46.7 & 8.94 & 9.80 & 9.68 \\
\hline & 4.23 & 6.45 & 57.8 & 9.43 & 11.30 & 19.89 \\
\hline & 4.40 & 6.45 & 62.4 & 10.09 & 11.60 & 15.02 \\
\hline \multirow{5}{*}{ 喜 } & 2.88 & 6.45 & 26.8 & 6.50 & 6.20 & -4.57 \\
\hline & 3.38 & 6.45 & 36.8 & 7.67 & 7.60 & -0.96 \\
\hline & 3.81 & 6.45 & 46.7 & 8.94 & 9.10 & 1.85 \\
\hline & 4.23 & 6.45 & 57.8 & 9.43 & 10.30 & 9.28 \\
\hline & 4.40 & 6.45 & 62.4 & 10.09 & 10.80 & 7.09 \\
\hline \multirow{5}{*}{ 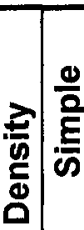 } & 2.88 & 6.45 & 26.8 & 6.50 & 6.60 & 1.59 \\
\hline & 3.38 & 6.45 & 36.8 & 7.67 & 8.00 & 4.26 \\
\hline & 3.81 & 6.45 & 46.7 & 8.94 & 9.70 & 8.56 \\
\hline & 4.23 & 6.45 & 57.8 & 9.43 & 10.80 & 14.59 \\
\hline & 4.40 & 6.45 & 62.4 & 10.09 & 11.20 & 11.06 \\
\hline \multirow{5}{*}{ 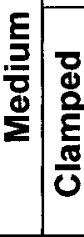 } & 2.88 & 6.45 & 26.8 & 6.50 & 6.40 & -1.49 \\
\hline & 3.38 & 6.45 & 36.8 & 7.67 & 7.80 & 1.65 \\
\hline & 3.81 & 6.45 & 46.7 & 8.94 & 9.30 & 4.09 \\
\hline & 4.23 & 6.45 & 57.8 & 9.43 & 10.70 & 13.53 \\
\hline & 4.40 & 6.45 & 62.4 & 10.09 & 11.00 & 9.07 \\
\hline \multirow{5}{*}{ 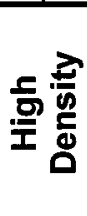 } & 2.88 & 6.45 & 26.8 & 6.50 & 6.60 & 1.59 \\
\hline & 3.38 & 6.45 & 36.8 & 7.67 & 7.80 & 1.65 \\
\hline & 3.81 & 6.45 & 46.7 & 8.94 & 9.20 & 2.97 \\
\hline & 4.23 & 6.45 & 57.8 & 9.43 & 10.40 & 10.34 \\
\hline & 4.40 & 6.45 & 62.4 & 10.09 & 11.00 & 9.07 \\
\hline
\end{tabular}


Table F3: Impact energy results for simulated events for preliminary aluminum impact simulations.

\begin{tabular}{|c|c|c|c|c|c|c|c|c|}
\hline & \multicolumn{3}{|c|}{ Experimental } & \multicolumn{3}{|c|}{ Simulation } & \multirow{2}{*}{$\begin{array}{c}\text { Percent } \\
\text { Difference in } \\
E_{a b s}\end{array}$} \\
\hline & & $\frac{E_{i m p}}{\mathbf{J}}$ & $\frac{E_{a b s}}{J}$ & $\begin{array}{c}\text { Percent } \\
\text { Absorbed }\end{array}$ & $\frac{E_{i m p}}{\mathrm{~J}}$ & $\frac{E_{a b s}}{J}$ & $\begin{array}{c}\text { Percent } \\
\text { Absorbed }\end{array}$ & \\
\hline \multirow{5}{*}{\multicolumn{2}{|c|}{ 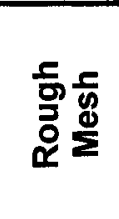 }} & 26.8 & 19.7 & 73.5 & 26.1 & 15.3 & $\bar{~} 58.6$ & 20.3 \\
\hline & & 36.8 & 27.4 & 74.5 & 36.6 & 23.3 & 63.7 & 14.5 \\
\hline & & 46.7 & 36.0 & 77.1 & 46.7 & 32.1 & 68.7 & 10.8 \\
\hline & & 57.8 & 45.6 & 78.9 & 56.9 & 41.8 & 73.5 & 6.9 \\
\hline & & 62.4 & 49.9 & 80.0 & 61.9 & 45.3 & 73.2 & 8.5 \\
\hline \multirow{5}{*}{\multicolumn{2}{|c|}{ 亭蛋 }} & 26.8 & 19.7 & 73.5 & 26.1 & 15.50 & 59.4 & 19.2 \\
\hline & & 36.8 & 27.4 & 74.5 & 36.6 & 23.75 & 64.9 & 12.8 \\
\hline & & 46.7 & 36.0 & 77.1 & 46.7 & 32.40 & 69.4 & 10.0 \\
\hline & & 57.8 & 45.6 & 78.9 & 56.9 & 42.00 & 73.8 & 6.4 \\
\hline & & 62.4 & 49.9 & 80.0 & 61.9 & 45.00 & 72.7 & 9.1 \\
\hline \multirow{10}{*}{ 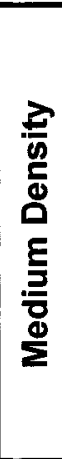 } & \multirow{5}{*}{$\frac{\frac{0}{0}}{\frac{E}{\omega}}$} & 26.8 & 19.7 & 73.5 & 26.1 & 15.75 & 60.3 & 17.9 \\
\hline & & 36.8 & 27.4 & 74.5 & 36.6 & 23.50 & 64.2 & 13.8 \\
\hline & & 46.7 & 36.0 & 77.1 & 46.7 & 32.00 & 68.5 & 11.1 \\
\hline & & 57.8 & 45.6 & 78.9 & 56.9 & 41.90 & 73.6 & 6.7 \\
\hline & & 62.4 & 49.9 & 80.0 & 61.9 & 45.00 & 72.7 & 9.1 \\
\hline & \multirow{5}{*}{$\begin{array}{l}\text { D } \\
\frac{0}{0} \\
\frac{E}{0} \\
\frac{\pi}{0}\end{array}$} & 26.8 & 19.7 & 73.5 & 26.1 & 14.25 & 54.6 & 25.7 \\
\hline & & 36.8 & 27.4 & 74.5 & 36.6 & 23.00 & 62.8 & 15.6 \\
\hline & & 46.7 & 36.0 & 77.1 & 46.7 & 31.50 & 67.5 & 12.5 \\
\hline & & 57.8 & 45.6 & 78.9 & 56.9 & 41.00 & 72.1 & 8.7 \\
\hline & & 62.4 & 49.9 & 80.0 & 61.9 & 44.80 & 72.4 & 9.5 \\
\hline \multirow{5}{*}{\multicolumn{2}{|c|}{ 옹 }} & 26.8 & 19.7 & 73.5 & 26.1 & 15.50 & 59.4 & 19.2 \\
\hline & & 36.8 & 27.4 & 74.5 & 36.6 & 23.80 & 65.0 & 12.7 \\
\hline & & 46.7 & 36.0 & 77.1 & 46.7 & 32.40 & 69.4 & 10.0 \\
\hline & & 57.8 & 45.6 & 78.9 & 56.9 & 42.00 & 73.8 & 6.4 \\
\hline & & 62.4 & 49.9 & 80.0 & 61.9 & 45.10 & 72.9 & 8.9 \\
\hline
\end{tabular}

Table F4: Results of the final impact simulations on aluminum 2024-T3.

\begin{tabular}{|c|c|c|c|c|c|c|c|c|c|c|c|c|c|}
\hline$\left(E_{\mathrm{mp}}\right)_{\text {num }}$ & $\left(E_{i m p}\right)_{\text {exp }}$ & \multicolumn{4}{|c|}{$E_{\text {abs }}(J)$} & \multicolumn{4}{|c|}{$d_{\text {perm }}(\mathrm{mm})$} & \multicolumn{4}{|c|}{$F_{\max }(k N)$} \\
\hline (J) & (J) & Thin & Thick & Solid & Exp. & Thin & Thick & Solid & Exp. & Thin & Thick & Solid & Exp. \\
\hline 25.1 & 26.8 & $\overline{115}$ & $\overline{14.3}$ & 14.1 & 19.7 & 6.8 & 6.25 & 5.08 & 6.5 & 6.4 & 6.3 & 6.67 & 5.7 \\
\hline 35.1 & 36.8 & 22 & 22.2 & 21.9 & 27.4 & 7.5 & 7.75 & 6.07 & 7.67 & 7.9 & 8.06 & 7.98 & 6.97 \\
\hline 44.8 & 46.7 & 32 & 30.3 & 30 & 36 & 9.8 & 9.25 & 7.47 & 8.94 & 8.9 & 9.24 & 9.15 & \begin{tabular}{|l|}
8.03 \\
\end{tabular} \\
\hline 54.8 & 57.8 & 40 & 38.6 & 38.5 & 45.6 & 11 & 10 & 8.4 & 9.43 & 10 & 10.2 & 10.3 & \begin{tabular}{|l|}
9.07 \\
\end{tabular} \\
\hline
\end{tabular}




\section{G - GLARE IMPACT SIMULATION RESULTS}

Table G1: Summary of GLARE simulation absorbed energy results.

\begin{tabular}{|c|c|c|c|c|c|c|c|}
\hline \multirow[t]{2}{*}{ Material } & \multirow{2}{*}{$\frac{\left(E_{i m p}\right)_{\exp }}{(\mathrm{J})}$} & \multirow{2}{*}{ 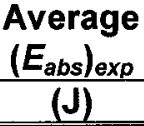 } & \multirow{2}{*}{$\begin{array}{c}\left(E_{i m p}\right)_{\text {sim }} \\
(\mathrm{J}) \\
\end{array}$} & \multicolumn{4}{|c|}{$\frac{\left(E_{a b s}\right)_{\operatorname{sim}}}{(\mathrm{J})}$} \\
\hline & & & & Tied & Simple & Corrected & UMAT \\
\hline \multirow{5}{*}{ 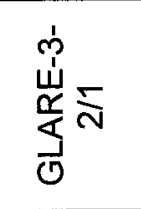 } & 16.12 & 10.67 & $\mathrm{~N} / \mathrm{A}$ & N/A & $\mathrm{N} / \mathrm{A}$ & $\mathrm{N} / \mathrm{A}$ & $N / A$ \\
\hline & 25.75 & 17.67 & 25 & 10.4 & 8.4 & 9.40 & 13.4 \\
\hline & 36.56 & 25.84 & 35 & 16.1 & 13.1 & 14.80 & 21 \\
\hline & 46.98 & 33.53 & 45 & 22.4 & 18.0 & 19.6 & 28 \\
\hline & 51.57 & 52.67 & 55 & 28.5 & 22.8 & 41 & 36 \\
\hline \multirow{5}{*}{ 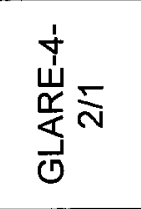 } & 26.52 & 17.36 & 25 & 9.46 & $\mathrm{~N} / \mathrm{A}$ & $\mathrm{N} / \mathrm{A}$ & $\mathrm{N} / \mathrm{A}$ \\
\hline & 36.86 & 24.88 & 35 & 14.81 & 12.4 & $N / A$ & 19.26 \\
\hline & 46.96 & 37.29 & 45 & 20.38 & 16.8 & $\mathrm{~N} / \mathrm{A}$ & 27 \\
\hline & 53.15 & 47.50 & 55 & 26.1 & 21.8 & $\mathrm{~N} / \mathrm{A}$ & 39.8 \\
\hline & 61.33 & 55.51 & 65 & N/A & $\mathrm{N} / \mathrm{A}$ & $\mathrm{N} / \mathrm{A}$ & N/A \\
\hline \multirow{5}{*}{ 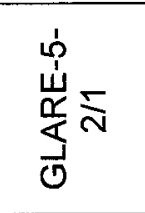 } & 24.78 & 16.72 & $N / A$ & N/A & $\mathrm{N} / \mathrm{A}$ & N/A & $\mathrm{N} / \mathrm{A}$ \\
\hline & 34.06 & 22.89 & 35 & 12.31 & $\mathrm{~N} / \mathrm{A}$ & N/A & 18.3 \\
\hline & 44.76 & 31.03 & 45 & 17.1 & $\mathrm{~N} / \mathrm{A}$ & $N / A$ & 24.7 \\
\hline & 54.29 & 38.21 & 55 & 22.1 & $\mathrm{~N} / \mathrm{A}$ & $\mathrm{N} / \mathrm{A}$ & 30.9 \\
\hline & 65.35 & 61.14 & 65 & 27.2 & $\mathrm{~N} / \mathrm{A}$ & $\mathrm{N} / \mathrm{A}$ & 39.29 \\
\hline
\end{tabular}

Table G2: Summary of GLARE simulation maximum force results.

\begin{tabular}{|c|c|c|c|c|c|c|c|}
\hline \multirow[t]{2}{*}{ Material } & \multirow{2}{*}{$\begin{array}{c}\left(E_{\text {imp }}\right)_{\text {exp }} \\
(J)\end{array}$} & \multirow{2}{*}{$\begin{array}{c}\text { Average } \\
\left(F_{\max }\right)_{\text {exp }} \\
(\mathrm{kN})\end{array}$} & \multirow{2}{*}{$\frac{\left(E_{i m p}\right)_{s i m}}{(\mathrm{~J})}$} & \multicolumn{4}{|c|}{$\frac{\left(F_{\max }\right)_{\operatorname{sim}}}{(\mathrm{kN})}$} \\
\hline & & & & Tied & Simple & Corrected & UMAT \\
\hline \multirow{5}{*}{ 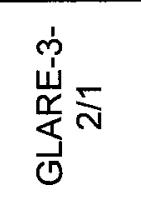 } & 16.12 & 4.09 & N/A & N/A & N/A & N/A & $\mathrm{N} / \mathrm{A}$ \\
\hline & 25.75 & 5.48 & 25 & 7.40 & 7.45 & 7.50 & 6.79 \\
\hline & 36.56 & 6.85 & 35 & 9.30 & 9.63 & 9.64 & 7.90 \\
\hline & 46.98 & 7.95 & 45 & 10.6 & 10.95 & 10.79 & 8.83 \\
\hline & 51.57 & 8.13 & 55 & 12.31 & 12.83 & 11.75 & 10.70 \\
\hline \multirow{5}{*}{ 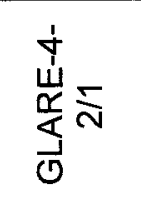 } & 26.52 & 5.69 & 25 & 7.61 & N/A & $\mathrm{N} / \mathrm{A}$ & N/A \\
\hline & 36.86 & 7.09 & 35 & 9.26 & 9.60 & N/A & 7.23 \\
\hline & 46.96 & 8.21 & 45 & 11.16 & 11.37 & N/A & 9.31 \\
\hline & 53.15 & 8.64 & 55 & 12.91 & 12.81 & N/A & 9.78 \\
\hline & 61.33 & 8.77 & 65 & N/A & $\mathrm{N} / \mathrm{A}$ & N/A & $\mathrm{N} / \mathrm{A}$ \\
\hline \multirow{5}{*}{ 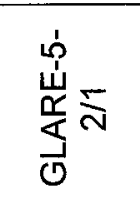 } & 24.78 & 5.67 & $\mathrm{~N} / \mathrm{A}$ & N/A & $\mathrm{N} / \mathrm{A}$ & N/A & $\mathrm{N} / \mathrm{A}$ \\
\hline & 34.06 & 6.98 & 35 & 8.75 & N/A & N/A & 8.84 \\
\hline & 44.76 & 8.26 & 45 & 10.16 & N/A & N/A & 10.2 \\
\hline & 54.29 & 9.37 & 55 & 10.95 & $\mathrm{~N} / \mathrm{A}$ & N/A & 11.6 \\
\hline & 65.35 & 10.37 & 65 & 12.63 & $\mathrm{~N} / \mathrm{A}$ & N/A & 13.3 \\
\hline
\end{tabular}


Table G3: Summary of GLARE permanent indentation results.

\begin{tabular}{|c|c|c|c|c|c|c|c|}
\hline \multirow[t]{2}{*}{ Material } & \multirow{2}{*}{$\begin{array}{c}\left(E_{\text {imp }}\right)_{\text {exp }} \\
(\mathrm{J}) \\
\end{array}$} & \multirow{2}{*}{\begin{tabular}{|c|}
$\begin{array}{c}\text { Average } \\
\left(d_{\text {perm }}\right)_{\text {exp }} \\
(\mathrm{mm})\end{array}$ \\
\end{tabular}} & \multirow{2}{*}{$\begin{array}{c}\left(E_{i m p}\right)_{s i m} \\
(\mathrm{~J}) \\
\end{array}$} & \multicolumn{4}{|c|}{$\begin{array}{c}\left(d_{\text {perm }}\right)_{\text {sim }} \\
(\mathrm{mm})\end{array}$} \\
\hline & & & & Tied & Simple & Corrected & UMAT \\
\hline \multirow{5}{*}{ 定 } & 16.12 & 5.28 & $\mathrm{~N} / \mathrm{A}$ & $N / A$ & $\mathrm{~N} / \mathrm{A}$ & N/A & $\mathrm{N} / \mathrm{A}$ \\
\hline & 25.75 & 7.03 & 25 & 5.4 & 2.78 & 5.24 & 7.00 \\
\hline & 36.56 & 8.08 & 35 & 7.00 & 3.75 & 5.89 & 8.23 \\
\hline & 46.98 & 8.98 & 45 & 8.00 & 4.75 & 6.68 & 9.63 \\
\hline & 51.57 & 15.23 & 55 & 8.43 & 5.00 & 7.75 & 11.00 \\
\hline \multirow{5}{*}{ 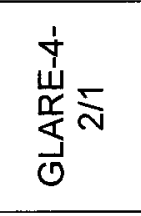 } & 26.52 & 6.40 & 25 & 4.87 & $\mathrm{~N} / \mathrm{A}$ & $\mathrm{N} / \mathrm{A}$ & N/A \\
\hline & 36.86 & 7.49 & 35 & 6.33 & 4.24 & $\mathrm{~N} / \mathrm{A}$ & 7.87 \\
\hline & 46.96 & 9.35 & 45 & 6.62 & 4.42 & N/A & 8.9 \\
\hline & 53.15 & 10.27 & 55 & 8.00 & 4.66 & N/A & 11.2 \\
\hline & 61.33 & 11.27 & 65 & N/A & N/A & N/A & N/A \\
\hline \multirow{5}{*}{ 定 } & 24.78 & 5.39 & N/A & N/A & $\mathrm{N} / \mathrm{A}$ & $\mathrm{N} / \mathrm{A}$ & $\mathrm{N} / \mathrm{A}$ \\
\hline & 34.06 & 6.57 & 35 & 5.1 & $\mathrm{~N} / \mathrm{A}$ & N/A & 7.15 \\
\hline & 44.76 & 7.89 & 45 & 5.76 & $\mathrm{~N} / \mathrm{A}$ & $\mathrm{N} / \mathrm{A}$ & 8.03 \\
\hline & 54.29 & 8.64 & 55 & 6.7 & $\mathrm{~N} / \mathrm{A}$ & N/A & 8.79 \\
\hline & 65.35 & 13.46 & 65 & 7.32 & $\mathrm{~N} / \mathrm{A}$ & N/A & 9.87 \\
\hline
\end{tabular}




\section{H - PARTIAL LIST OF PUBLICATIONS RESULTING FROM THE FML PROJECT}

Refereed Journal Publications:

Laliberté, J., Poon, C., Straznicky, P.V. and Fahr, A. "Applications of Fibre-Metal Laminates in Airframe Structures," Polymer Composites, v. 21, n. 4, August 2000.

Fahr, A., Chapman, C. E., Laliberté, J. and Poon, C., "Non-Destructive Evaluation Methods for Damage Assessment in Fiber-Metal Laminates," Polymer Composites, v. 21, n. 4, August 2000.

Laliberté, J., Poon, C., Straznicky, P.V. and Fahr, A. "Post-Impact Fatigue in FiberMetal Laminates," International Journal of Fatigue, 2002, v. 24, pp. 249-256.

Conferences and Workshops:

Laliberté, J., Straznicky, P.V. and Poon, C. "Numerical Modelling of Low-Velocity Impact Damage in Fibre-Metal Laminates," 2002 ICAS Congress, Toronto, Canada, September 2002 .

Laliberté, J., Straznicky, P.V. and Poon, C. "Mode-I Delamination in Cross-Ply FibreMetal Laminates," ICF-10, Honolulu, USA, December 2001.

Laliberté, J., Straznicky, P.V. and Poon, C. "Post Impact Shear Behaviour in GLARE," $3^{\text {rd }}$ Canadian-International Composites Conference, Montreal, Canada, August 2001.

Laliberté, J., Poon, C. Straznicky, P.V., Fawaz. Z. and McCuaig, K. "Post-Impact Biaxial Fatigue Crack Growth in GLARE," $21^{\text {st }}$ Symposium of the International Committee on Aeronautical Fatigue, Toulouse, France, June 2001.

Fahr, A., Chapman, C.E., Laliberté, J., and Poon, C., "Non-Destructive Evaluation of Fiber-Metal Laminates" $7^{\text {th }}$ International Conference on Composites Engineering, Denver, USA, July 2-8 2000.

Poon, C., Laliberté, J. and Straznicky, P.V. "Review of Fibre-Metal-Laminate Technology," $3^{\text {rd }}$ Joint Canada-Japan Workshop on Composites, Kyoto, Japan, 2000. 
Laliberté, J., Poon, C., Straznicky, P.V. and Fahr, A. "Post-Impact Fatigue in FiberMetal Laminates," $2^{\text {nd }}$ International Conference on the Fatigue of Composites, Williamsburg, USA, June 2000.

Laliberté, J., Poon, C. and Straznicky, P.V. "Application of Fibre-Metal Laminates in Airframe Structures," SPE - Polymer Composites '99, Quebec City, Canada, October 1999.

Fahr, A., Chapman, C.E., Forsyth, D.F., Laliberté, J. and Poon, C., "Non-Destructive Evaluation Methods for Damage Assessment in Fibre-Metal Laminates," SPE-Polymer Composites '99, Quebec City, Canada, October 1999.

Laliberté, J., Poon, C. and Straznicky, P.V. "Low-Velocity Impact Damage in GLARE Fibre-Metal Laminates," ICCM-12, Paris, France, July 1999.

Laliberté, J. and Poon, C. "Durability of Hybrid Fibre Metal Laminates," contribution to the International Committee on Aeronautical Fatigue 1999 Summary.

Laliberté, J., Poon, C. and Straznicky, P.V., "Impact Damage Tolerance in Composites and Fibre Metal Laminates," Canada-Taiwan Symposium on Aircraft Materials Testing, Maintenance and Repair, Ottawa, Canada, April 1999.

\section{Internal NRC Reports}

Laliberté, J., Poon, C. and Straznicky, P.V. "Durability Testing of Fibre-Metal Laminates," IAR-SMPL, Laboratory Report LTR-ST-2231, October 1999.

McCuaig, K., Laliberté, J., Poon, C. and Straznicky, P.V. "Post-Impact, Off-Axis Fatigue of Glare-4 3/2 Variant and Aluminum," IAR-SMPL, Laboratory Memorandum, November 2001 [under review].

Price, A., Laliberté, J. and Poon, C. "Tensile Testing of GLARE-3," IAR-SMPL Laboratory Memorandum, March 2002 [under review]. 